\title{
Frequency of Eating and Cardiovascular Disease: A Systematic Review
}

\author{
2020 Dietary Guidelines Advisory Committee, \\ Frequency of Eating Subcommittee \\ Published date: July 15, 2020
}

Nutrition Evidence Systematic Review

Center for Nutrition Policy and Promotion

Food and Nutrition Service

U.S. Department of Agriculture

Braddock Metro Center II

1320 Braddock Place

Alexandria, Virginia 22314 
This systematic review was conducted by the 2020 Dietary Guidelines Advisory Committee in collaboration with the Nutrition Evidence Systematic Review (NESR) team at the Center for Nutrition Policy and Promotion, Food and Nutrition Service, U.S. Department of Agriculture (USDA). All systematic reviews from the 2020 Advisory Committee Project are available on the NESR website: https://nesr.usda.gov/2020-dietaryguidelines-advisory-committee-systematic-reviews.

Conclusion statements drawn as part of this systematic review describe the state of science related to the specific question examined. Conclusion statements do not draw implications, and should not be interpreted as dietary guidance. This portfolio provides the complete documentation for this systematic review. A summary of this review is included in the 2020 Advisory Committee's Scientific Report available at www.DietaryGuidelines.gov.

The contents of this document may be used and reprinted without permission. Endorsements by NESR, the Center for Nutrition Policy and Promotion, the Food and Nutrition Service, or the USDA of derivative products developed from this work may not be stated or implied.

Suggested citation for this systematic review: 2020 Dietary Guidelines Advisory Committee and Nutrition Evidence Systematic Review Team. Frequency of Eating and Cardiovascular Disease: A Systematic Review. 2020 Dietary Guidelines Advisory Committee Project. Alexandria, VA: U.S. Department of Agriculture, Food and Nutrition Service, Center for Nutrition Policy and Promotion, July 2020. Available at: https://nesr.usda.gov/2020-dietary-guidelines-advisory-committee-systematic-reviews.

Related citation: Dietary Guidelines Advisory Committee. 2020. Scientific Report of the 2020 Dietary Guidelines Advisory Committee: Advisory Report to the Secretary of Agriculture and the Secretary of Health and Human Services. U.S. Department of Agriculture, Agricultural Research Service, Washington, DC.

In accordance with Federal civil rights law and USDA civil rights regulations and policies, the USDA, its Agencies, offices, and employees, and institutions participating in or administering USDA programs are prohibited from discriminating based on race, color, national origin, religion, sex, gender identity (including gender expression), sexual orientation, disability, age, marital status, family/parental status, income derived from a public assistance program, political beliefs, or reprisal or retaliation for prior civil rights activity, in any program or activity conducted or funded by USDA (not all bases apply to all programs). Remedies and complaint filing deadlines vary by program or incident.

Persons with disabilities who require alternative means of communication for program information (e.g., Braille, large print, audiotape, American Sign Language, etc.) should contact the responsible Agency or USDA's TARGET Center at (202) 720-2600 (voice and TTY) or contact USDA through the Federal Relay Service at (800) 877-8339. Additionally, program information may be made available in languages other than English.

To file a program discrimination complaint, complete the USDA Program Discrimination Complaint Form, AD3027, found online at How to File a Program Discrimination Complaint and at any USDA office or write a letter addressed to USDA and provide in the letter all of the information requested in the form. To request a copy of the complaint form, call (866) 632-9992. Submit your completed form or letter to USDA by: (1) mail: U.S. Department of Agriculture, Office of the Assistant Secretary for Civil Rights, 1400 Independence Avenue, SW, Washington, D.C. 20250-9410; (2) fax: (202) 690-7442; or (3) email: program.intake@usda.gov.

USDA is an equal opportunity provider, employer, and lender. 


\section{ACKNOWLEDGEMENTS}

Frequency of Eating Subcommittee:

- Steven Heymsfield, MD, Louisiana State University, Pennington Biomedical Research Center, Subcommittee Chair

- Carol Boushey, PhD, MPH, RD, University of Hawaii

- Heather Leidy, PhD, University of Texas at Austin

- Richard Mattes, PhD, MPH, RD, Purdue University

- Ronald Kleinman, MD, Massachusetts General Hospital, Harvard Medical School, Vice-Chair of the 2020 Dietary Guidelines Advisory Committee

\section{Nutrition Evidence Systematic Review (NESR) Team:}

- Emily Callahan, MS, Analyst, Office of Nutrition Guidance and Analysis (ONGA), Center for Nutrition Policy and Promotion (CNPP), Food and Nutrition Service (FNS), U.S. Department of Agriculture (USDA)

- Gisela Butera, MLIS, MEd, Systematic Review Librarian, Panum Group.

- Nancy Terry, MLS, Biomedical Librarian, National Institutes of Health (NIH) Library, U.S. Department of Health and Human Services (HHS)

- Julie Obbagy, PhD, RD, Project Lead, ONGA, CNPP, FNS, USDA

\section{Federal Liaisons:}

- Ashley Vargas, PhD, MPH, RDN, FAND, Eunice Kennedy Shriver National Institute of Child Health and Human Development, NIH, HHS

- Meghan Adler, MS, RD, FAND, ONGA, CNPP, FNS, USDA

- Rebecca Maclsaac, MS, RD, Division of Prevention Science (DPS), Office of Disease Prevention and Health Promotion (ODPHP), Office of the Assistant Secretary for Health (OASH), HHS

- Julia Quam, MSPH, RDN, DPS, ODPHP, OASH, HHS

\section{Project Leadership:}

- Eve Essery Stoody, PhD, Designated Federal Officer and Director, ONGA, CNPP, FNS, USDA

- Janet de Jesus, MS, RD, Nutrition Advisor, ODPHP, OASH, HHS

- Colette Rihane, MS, RD, Director, ONGA, CNPP, FNS, USDA (through October 2019)

USDA and HHS implemented a process to identify topics and scientific questions to be examined by the 2020 Dietary Guidelines Advisory Committee. The Committee conducted its review of evidence in subcommittees for discussion by the full Committee during its public meetings. The role of the Committee members involved establishing all aspects of the protocol, which presented the plan for how they would examine the scientific evidence, including the inclusion and exclusion criteria; reviewing all studies that met the criteria they set; deliberating on the body of evidence

i Under contract with the Food and Nutrition Service, United States Department of Agriculture. 
for each question; and writing and grading the conclusion statements to be included in the scientific report the 2020 Committee submitted to USDA and HHS. The NESR team with assistance from Federal Liaisons and Project Leadership, supported the Committee by facilitating, executing, and documenting the work necessary to ensure the reviews were completed in accordance with NESR methodology. More information about the 2020 Dietary Guidelines Advisory Committee, including the process used to identify topics and questions, can be found at www.DietaryGuidelines.gov. More information about NESR can be found at NESR.usda.gov.

The Committee and NESR staff thank USDA's Agricultural Research Service for coordinating the peer review of this systematic review, and the Federal scientist peer reviewers for their time and expertise.

FUNDING SOURCE: United States Department of Agriculture, Food and Nutrition Service, Center for Nutrition Policy and Promotion, Alexandria, VA 


\section{TABLE OF CONTENTS}

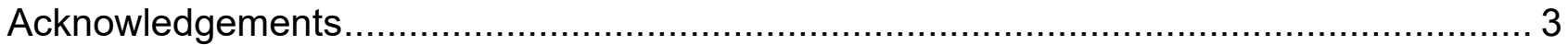

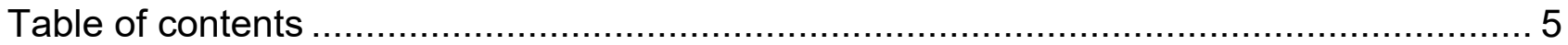

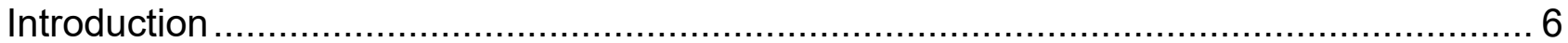

What is the relationship between the frequency of eating and risk of cardiovascular

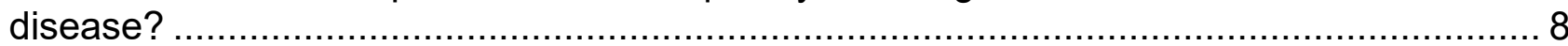

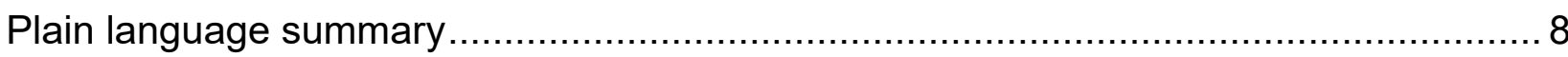

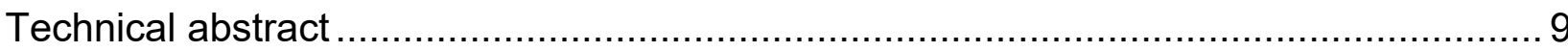

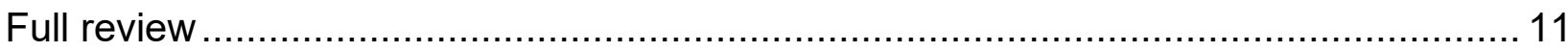

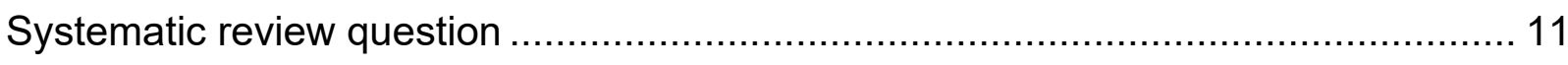

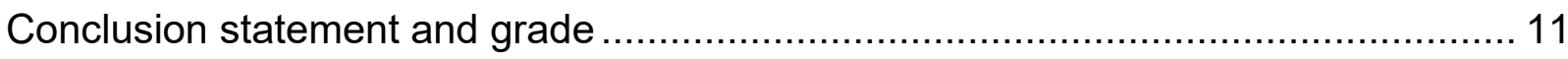

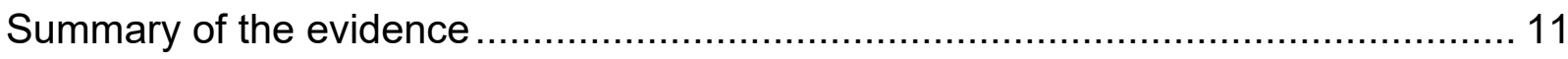

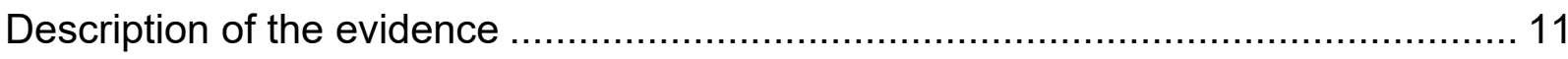

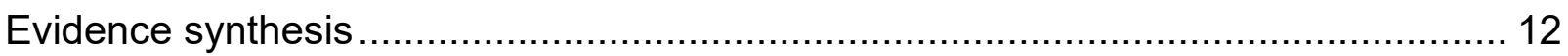

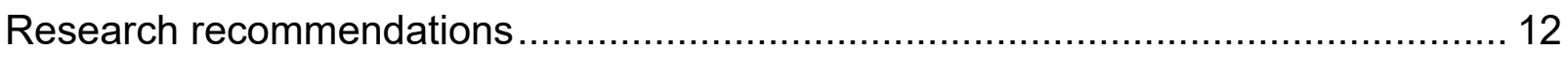

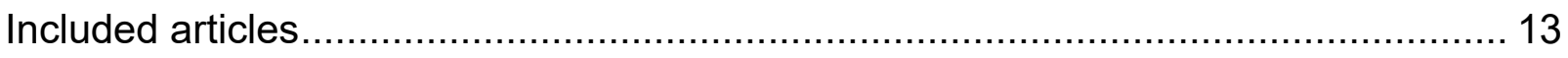

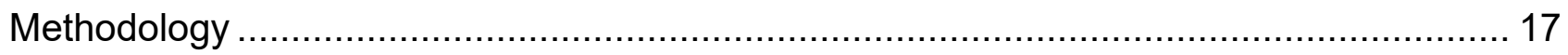

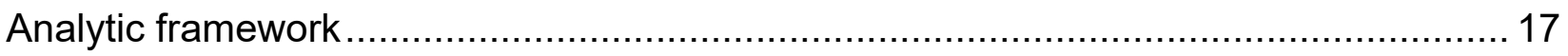

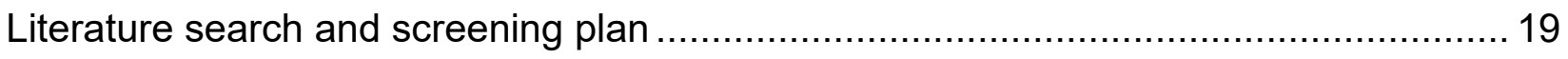

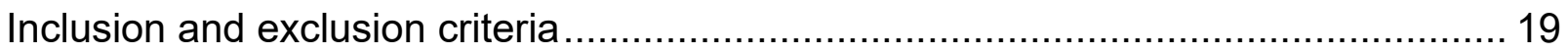

Electronic databases and search terms......................................................... 22

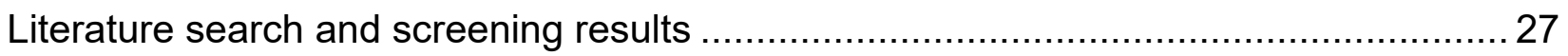

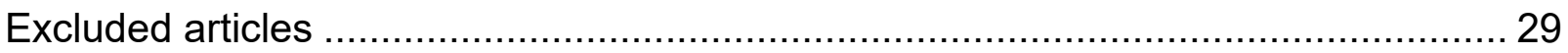

Table 1. Description of studies included in the systematic review to examine the relationship between frequency of eating and cardiovascular disease ........................... 14 Table 2. Risk of bias for observational studies examining the frequency of eating and

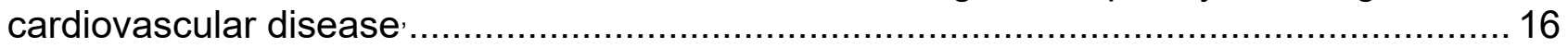

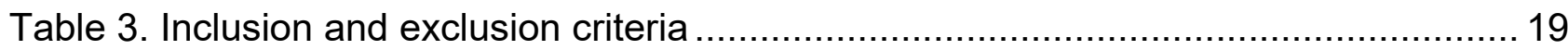

Table 4. Articles excluded after full text screening with rationale for exclusion...................29

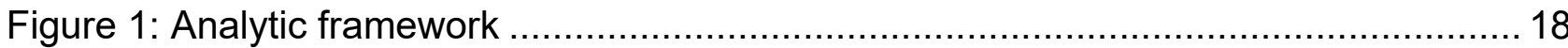

Figure 2: Flow chart of literature search and screening results................................... 28 
This document describes a systematic review conducted to answer the following question: What is the relationship between the frequency of eating and cardiovascular disease? This systematic review was conducted by the 2020 Dietary Guidelines Advisory Committee, supported by USDA's Nutrition Evidence Systematic Review (NESR).

More information about the 2020 Dietary Guidelines Advisory Committee is available at the following website: www.DietaryGuidelines.gov.

NESR specializes in conducting food- and nutrition-related systematic reviews using a rigorous, protocol-driven methodology. More information about NESR is available at the following website: NESR.usda.gov.

NESR's systematic review methodology involves developing a protocol, searching for and selecting studies, extracting data from and assessing the risk of bias of each included study, synthesizing the evidence, developing conclusion statements, grading the evidence underlying the conclusion statements, and recommending future research. A detailed description of the systematic reviews conducted for the 2020 Dietary Guidelines Advisory Committee, including information about methodology, is available on the NESR website: https://nesr.usda.gov/2020-dietary-guidelines-advisory-committee-systematic-reviews. In addition, starting on page 17, this document describes the final protocol as it was applied in the systematic review. A description of and rationale for modifications made to the protocol are described in the 2020 Dietary Guidelines Advisory Committee Report, Part D: Chapter 13. Frequency of Eating. 


\section{List of abbreviations}

\begin{tabular}{ll}
\hline Abbreviation & Full name \\
\hline CHD & Coronary heart disease \\
\hline CNPP & Center for Nutrition Policy and Promotion \\
\hline CVD & Cardiovascular disease \\
\hline DPS & Division of Prevention Science \\
\hline FFQ & Food frequency questionnaire \\
\hline F/U & Follow-up \\
\hline FNS & Food and Nutrition Service \\
\hline FoE & Frequency of eating \\
\hline HHS & United States Department of Health and Human Services \\
\hline NESR & Nutrition Evidence Systematic Review \\
\hline NIH & National Institute of Health \\
\hline ODPHP & Office of Disease Prevention and Health Promotion \\
\hline ONGA & Office of Nutrition Guidance and Analysis \\
\hline USDA & United States Department of Agriculture \\
\hline
\end{tabular}




\section{WHAT IS THE RELATIONSHIP BETWEEN THE FREQUENCY OF EATING AND RISK OF CARDIOVASCULAR DISEASE?}

\section{PLAIN LANGUAGE SUMMARY}

What is the question?

- The question is: What is the relationship between the frequency of eating and risk of cardiovascular disease?

What is the answer to the question?

- Insufficient evidence is available to determine the relationship between the frequency of eating and cardiovascular disease.

Why was this question asked?

- This important public health question was identified by the U.S. Departments of Agriculture (USDA) and Health and Human Services (HHS) to be examined by the 2020 Dietary Guidelines Advisory Committee.

How was this question answered?

- The 2020 Dietary Guidelines Advisory Committee, Frequency of Eating Subcommittee conducted a systematic review to answer this question with support from the Nutrition Evidence Systematic Review (NESR) team.

- Frequency of eating was defined as the number of daily eating occasions.

What is the population of interest?

- The population of interest was generally healthy children through older adults (ages 2 and older).

\section{What evidence was found?}

- This review identified 2 articles that met the inclusion criteria.

- Inconsistent results from 2 prospective cohort studies with critical limitations, were insufficient to draw conclusions.

How up-to-date is this systematic review?

- This review searched for studies from January 2000 to September 2019. 


\section{TECHNICAL ABSTRACT}

\section{Background}

- This important public health question was identified by the U.S. Departments of Agriculture (USDA) and Health and Human Services (HHS) to be examined by the 2020 Dietary Guidelines Advisory Committee.

- The 2020 Dietary Guidelines Advisory Committee, Frequency of Eating Subcommittee conducted a systematic review to answer this question with support from the Nutrition Evidence Systematic Review (NESR) team.

- The goal of this systematic review was to examine the following question: What is the relationship between the frequency of eating and risk of cardiovascular disease?

\section{Conclusion statement and grade}

- Insufficient evidence is available to determine the relationship between the frequency of eating and cardiovascular disease. (Grade: Grade not assignable)

\section{Methods}

- Literature search was conducted using 4 databases (PubMed, Cochrane, Embase, and $\mathrm{CINAHL)} \mathrm{to} \mathrm{identify} \mathrm{articles} \mathrm{that} \mathrm{evaluated} \mathrm{an} \mathrm{intervention} \mathrm{or} \mathrm{exposure} \mathrm{of} \mathrm{the}$ frequency of eating and the outcome of cardiovascular disease. A manual search was also conducted to identify articles that may not have been included in the electronic databases searched. Articles were screened by two authors independently for inclusion based on pre-determined criteria.

- Data extraction and risk of bias assessment were conducted for each included study, and both were checked for accuracy. The Committee qualitatively synthesized the body of evidence to inform development of a conclusion statement(s), and graded the strength of evidence using pre-established criteria for risk of bias, consistency, directness, precision, and generalizability.

\section{Summary of the evidence}

- This systematic review was undertaken to examine the relationship between the frequency of eating and cardiovascular disease.

- This review identified 2 prospective cohort studies published between January, 2000 and September, 2019 that met the inclusion criteria for this systematic review.

- The studies were inconsistent in terms of how they defined and examined frequency of eating, the outcomes they examined, and in their reported results:

- One included study reported that a higher eating frequency at baseline was associated with lower blood pressure after a 5-year follow-up.

- One included study reported no association between eating frequency at baseline and risk of coronary heart disease after a 16-year follow-up.

- Critical limitations were identified within the body of evidence:

- Weak study designs were used to explore this question.

- Eating frequency was measured only at baseline, leading to concern that changes in exposure status may have occurred over the follow-up time periods.

- The amount of attrition was unknown.

- The study showed several risks of bias. 
- Inconsistent outcomes were included across studies with respect to relative risks and $95 \%$ confidence intervals for CHD, systolic blood pressure, diastolic blood pressure, and hypertension.

- Water consumption was not explicitly mentioned in either included study.

- Due to the small body of evidence with inconsistency in design and reported results and several limitations, the Committee determined that the evidence was insufficient to draw conclusions about the relationship between frequency of eating and cardiovascular disease. 


\section{FULL REVIEW}

\section{Systematic review question}

What is the relationship between the frequency of eating and risk of cardiovascular disease?

\section{Conclusion statement and grade}

Insufficient evidence is available to determine the relationship between the frequency of eating and cardiovascular disease. (Grade: Grade not assignable)

\section{Summary of the evidence}

- This systematic review was undertaken to examine the relationship between the frequency of eating and cardiovascular disease.

- This review identified 2 prospective cohort studies published between January, 2000 and September, 2019 that met the inclusion criteria for this systematic review. ${ }^{1,2}$

- The studies were inconsistent in terms of how they defined and examined frequency of eating, the outcomes they examined, and in their reported results:

- One included study reported that a higher eating frequency at baseline was associated with lower blood pressure after a 5-year follow-up.

- One included study reported no association between eating frequency at baseline and risk of coronary heart disease after a 16-year follow-up.

- Critical limitations were identified within the body of evidence:

- Weak study designs were used to explore this question.

- Eating frequency was measured only at baseline, leading to concern that changes in exposure status may have occurred over the follow-up time periods.

- The amount of attrition was unknown.

- The study showed several risks of bias.

- Inconsistent outcomes were included across studies with respect to relative risks and $95 \%$ confidence intervals for $\mathrm{CHD}$, systolic blood pressure, diastolic blood pressure, and hypertension.

- Water consumption was not explicitly mentioned in either included study.

- Due to the small body of evidence with inconsistency in design and reported results and several limitations, the Committee determined that the evidence was insufficient to draw conclusions about the relationship between frequency of eating and cardiovascular disease.

\section{Description of the evidence}

This systematic review included 2 articles that addressed the relationship between the frequency of eating and cardiovascular disease, published between January 2000 and September 2019.1,2 Basic characteristics of the studies are shown in Table 1. The studies were both prospective cohort studies. One used data from the Health Professionals Follow-up Study conducted in the United States and one was conducted in Greece.

Both studies were conducted in adults, one in an all-male, majority White population with an mean age of 58 years $^{1}$ and the other study was $45 \%$ female with an average 
age of 47.8 years in a Greek population. ${ }^{2}$ Average BMI was about 25 and $26 \mathrm{~kg} / \mathrm{m}^{2}$

and average habitual intakes were 3.5 and 5 eating occasions per day, respectively. ${ }^{1,2}$

In Cahill et al, ${ }^{1}$ the authors looked at the frequency of eating occasions at baseline in relation to risk of coronary heart disease over a 16-year follow-up. In Karatzi et al, ${ }^{2}$ the authors assessed the relationship between frequency of eating occasions at baseline and systolic blood pressure, diastolic blood pressure, and risk of hypertension after 5 years of follow-up.

\section{Evidence synthesis}

Cahill et $\mathrm{al}^{1}$ reported no association in males with a mean age of 58 years, between frequency of eating at baseline and risk of coronary heart disease over a 16-year follow-up. Karatzi et al ${ }^{2}$ reported an inverse association in adults between eating frequency at baseline and systolic and diastolic blood pressure changes, and risk of hypertension after a 5-year follow-up. Results are shown in Table 1.

Both included studies had several limitations (Table 2). Both included studies had unknown attrition rates and did not account for missing data or loss to follow-up. In addition, while habitual eating frequency was assessed, it was only assessed once at baseline, leading to concerns that changes in exposure status could have occurred over the study follow-ups. Data collection methods assessing frequency of eating and the definitions used for eating occasions were inconsistent between the two studies. Cahill et $\mathrm{al}^{1}$ added a question to a food frequency questionnaire (FFQ) in which participants were asked to indicate if they ate at different times of the day anchored by meals (before breakfast, breakfast, between breakfast and lunch, lunch, between lunch and dinner, dinner, between dinner and bedtime, or after going to bed). This method was focused on eating and beverages were likely not considered in this assessment method. Karatzi et $\mathrm{al}^{2}$ used a 3-day dietary record and defined an eating occasion as any time when food or beverages were consumed at least 15 minutes apart. Neither study explicitly mentioned the consideration of water within the analyses. The differences in these methods made it difficult to compare results from the studies.

The 2 studies included in this systematic review were designed to examine the relationship between frequency of eating and cardiovascular disease, and were generalizable to the U.S. population. However, they were inconsistent in their definitions and examination of frequency of eating, the outcomes considered, and the reported results. In addition, the included studies had multiple critical risks of bias due to their design and conduct. Publication bias is a concern with this body of evidence given that there were few studies and only one study reported a significant association. Therefore, the evidence was insufficient to be able to draw conclusions about the relationship between the frequency of eating and cardiovascular disease.

\section{Research recommendations}

- More controlled trials are needed that assess the frequency of eating and various outcomes.

- Future studies should develop a consistent definition of an ingestive event that includes eating and drinking and methods to quantify it. 
- Future studies should document the frequency of water consumption.

- In future studies, collection of ingestive frequency data should:

- Report number of ingestive events across 24 hours.

- Collect a minimum of 3 days of ingestive event data on at least 2 discrete occasions to allow assessment of estimate reliability.

- Future studies should report information on food insecurity in relation to frequency of eating to allow isolation of voluntary versus involuntary eating frequency effects.

- Future research should report key confounders and other factors to be considered, such as:

○ Sex, Age, Race/ethnicity, Habitual eating frequency, Smoking, Anthropometry

- Socioeconomic status, Physical activity, Cultural practices, Total energy intake, Diet energy density, Energy state of the diet (restriction/surplus), Energy balance (total energy intake/total energy expenditure), Chrononutrition factors (time of day, (consistency of) habitual eating frequency, and duration between ingestive events and/or ingestive periods), Portion size, Macronutrient content, Location of eating occasion, Eating environment (who you eat with, work/school/exercise schedule), Holiday eating (seasonal), Sleep schedule (shift work), Secondary eating, Dentition, Hydration status, Pregnancy status, Pubertal status, Menopausal status, Biochemical changes

\section{Included articles}

1. Cahill LE, Chiuve SE, Mekary RA, et al. Prospective study of breakfast eating and incident coronary heart disease in a cohort of male US health professionals. Circulation. 2013;128(4):337-343. doi:10.1161/circulationaha.113.001474

2. Karatzi K, Georgiopoulos G, Yannakoulia M, et al. Eating frequency predicts new onset hypertension and the rate of progression of blood pressure, arterial stiffness, and wave reflections. J Hypertens. 2016;34(3):429-437.

doi:10.1097/hjh.0000000000000822 
Table 1. Description of studies included in the systematic review to examine the relationship between frequency of eating and cardiovascular disease.i

$\begin{array}{llll}\text { Study and population characteristics } & \begin{array}{l}\text { Exposure, Comparator and } \\ \text { Outcome(s) }\end{array} & \text { Results } & \text { Key Confounders and Study } \\ \text { Limitations }\end{array}$

\section{Cahill, 2013 1}

Prospective Cohort Study, Health Professionals Follow-Up Study, United States; Analytic N=26902

\section{Participant characteristics:}

Key Confounders:

- $\quad$ Age: $~ 58 y(46-81 y)$

- Sex: $0 \%$ female

- Race/Ethnicity: 97\% White

- Habitual eating frequency: $\sim 3.5 \mathrm{EOs} / \mathrm{d}, \sim 87 \%$ breakfast consumers

- Smoking: $7 \%$ current smokers

- Anthropometry: $\sim 25 \mathrm{~kg} / \mathrm{m}^{2}$

Reported Other Factors to be Considered:

- SES: Married $~ 90 \%$

- $\quad$ Physical activity: $~ 37 \mathrm{MET} / \mathrm{h} / \mathrm{wk}, \sim 11 \%$ watch $>21 \mathrm{~h}$ tv/wk

- $\quad$ Sleep: $<7 \mathrm{hr} / 24 \mathrm{~h} \sim 16 \%$

- Drink alcohol $76 \%$

- Total energy intake: $1950 \mathrm{kcal} / \mathrm{d}$

- Health: diabetes $\sim 4 \%$; hypertension $\sim 26 \%$; hypercholesterolemia $\sim 34 \%$

Excluded from selection into study or analysis: Females, did not complete the baseline FFQ, had implausible energy intakes ( $>4200$ or $<800 \mathrm{kcal} / \mathrm{d}$ ), had left $>70$ food items blank, or did not answer the meal-frequency questions, diagnosed with diabetes, cardiovascular disease, and cancer (except for nonmelanoma skin cancer)

\section{Exposure of interest:}

Frequency of eating occasions:

$1-2,3,4-5, \geq 6$ times per day

Assessment method and timing:

FFQ, collected in 1992; indicate eating before breakfast, breakfast, between breakfast and lunch, lunch, between lunch and dinner, dinner, between dinner and bedtime, or after going to bed (question focused on eating, so likely beverages were not considered)

Treatment of beverages: Likely not included

\section{Outcomes and assessment} methods:

Coronary Heart Disease (CHD): Non-fatal myocardial infarction or fatal CHD were self-reported and confirmed based on medical record review; every $2 y$ for $16 y$
TEI adjusted: Yes

CHD:

- 1-2 times/d ( $=150):$ RR: 1.08 $95 \% \mathrm{Cl}: 0.90,1.29$

- 3 times/d $(n=728):$ RR: 1.00

- 4-5 times/d ( $n=611)$ : RR: 1.00 $95 \% \mathrm{Cl}: 0.89,1.11$

- $\geq 6$ times/d ( $=38):$ RR: $1.14,95 \%$

$$
\mathrm{Cl}: 0.81,1.61
$$

- $p$-trend = NS

Summary: Frequency of eating at men with a mean age 58y was not significantly associated with risk of CHD after $16 y \mathrm{f} / \mathrm{u}$.

\section{Model Adjustments:}

- Key confounders: age, sex, race/ethnicity, physical activity, smoking, anthropometry

- Other factors to be considered: SES, family history of MI, energy intake, sleep

- Other: alcohol intake, diet quality, marital status, physical exam in last $2 y$, television watching, diagnosis with diabetes, hypertension, or hypercholesterolemia, breakfast, late night eating, stress, antidepressant medication, daily number of cigarettes among smokers, bodyweight change and specific dietary components such as folate, whole grains, fiber or saturated fat

\section{Limitations:}

- Selection criteria likely related to FoE and CVD

- FoE data only assessed at baseline with a $16 y$ $\mathrm{f} / \mathrm{u}$ leading to a concern that changes in exposure status occurred over $\mathrm{f} / \mathrm{u}$

- FoE assessment method not validated

- High attrition rate

- Beverages and water was not mentioned in FoE assessment

- FoE data collection from 1992 and may not be applicable to current populations and food supply

Funding sources: $\mathrm{NIH}$, Canadian Institutes of Health Research

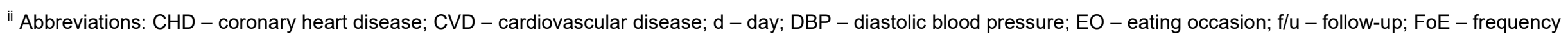

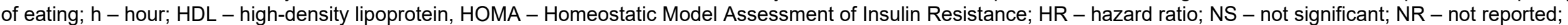
SBP - systolic blood pressure; SD - standard deviation; SE - standard error; SES - socioeconomic status; TEI - total energy intake, y - year 


\section{Study and population characteristics}

Karatzi, 2016²

Prospective Cohort Study, Greece; Analytic N=115

Participant characteristics:

\section{Key Confounders:}

- $\quad$ Age: $47.8 \mathrm{y}$

- Sex: $45 \%$ female

- Race/Ethnicity: NR

- Habitual eating frequency, median, IQR: 15, 13-18 $\mathrm{EOs} / 3 \mathrm{~d}$

- Smoking: $40 \%$

- Anthropometry: BMI: $26.2 \mathrm{~kg} / \mathrm{m}^{2}$; waist circumference: $89.5 \mathrm{~cm}$; hip circumference: $102 \mathrm{~cm}$; waist to hip ratio: 0.854

\section{Reported Other Factors to be Considered:}

- Total energy intake: $\sim 1823 \mathrm{kcal}$

- Macronutrient content: carbohydrate energy $~ 39.8 \%$; Lipid energy $\sim 41.3 \%$; protein energy $\sim 38.2 \%$;

Excluded from selection into study or analysis: history of coronary artery disease, diabetes, liver or endocrine

diseases, autoimmune disease, cancer, or active infection; alcohol consumption $\geq 30 \mathrm{~g} / \mathrm{d}$; dieting at baseline

\section{Exposure, Comparator and} Outcome(s)

\section{Exposure of interest:}

Frequency of EOs: $1-5, \geq 6$ per day

Assessment method and timing: $3 \mathrm{~d}$ food records on 2

consecutive weekdays and 1

weekend day at baseline; eating

frequency: number of EOs/d

(mean of $3 \mathrm{~d}$ assessments);

EO: any EO when food or drink

was consumed at least $15 \mathrm{~min}$

apart (may include alcohol, soft

drinks, kcal-free meals, or

coffees and teas eaten in the

absence of food.

Treatment of non-caloric

beverages: Included as an EO

although water not mentioned.

\section{Outcomes and assessment} methods:

Cardiovascular disease: Blood pressure was measured

(average of 2 measures, $1 \mathrm{~min}$ apart), at baseline and $5 y$ follow-up; Hypertension was defined as systolic blood pressure $>140 \mathrm{mmHg}$ and/or diastolic blood pressure > $90 \mathrm{mmHg}$. Participants fasted for $12 \mathrm{~h}$ and abstained from alcohol, caffeine, and smoking for $1 \mathrm{~d}$ before assessment at lab.

\section{Results}

Key Confounders and Study Limitations

\section{TEI adjusted: Yes}

CVD:

- SBP: HR 0.366, $p=0.05$

- DBP: $0.452, p=0.02$

- Hypertension: HR 5.4, p=0.004

Summary: Higher eating frequency at baseline was associated with lower systolic blood pressure and diastolic blood pressure changes, and decreased risk of hypertension after a 5-year follow-up.
Model Adjustments:

- Key confounders: age, sex, smoking, BMI

- Other factors to be considered: total energy intake

- Other: HDL, HR, HOMA

Limitations:

- Race/ethnicity (study took place in Greece)

was not accounted for in the analysis

- Selection criteria likely related to FoE and CVD

- FoE data only assessed at baseline with a 5y

$\mathrm{f} / \mathrm{u}$ leading to a concern that changes in

exposure status occurred over $\mathrm{f} / \mathrm{u}$

- High attrition rate

- Water not mentioned in data collection

Funding sources: NR 
Table 2. Risk of bias for observational studies examining the frequency of eating and cardiovascular disease ${ }^{i i i, i v}$

\begin{tabular}{|l|c|c|c|c|c|c|c|}
\hline & Confounding & $\begin{array}{c}\text { Selection of } \\
\text { participants }\end{array}$ & $\begin{array}{c}\text { Classification } \\
\text { of exposures }\end{array}$ & $\begin{array}{c}\text { Deviations } \\
\text { from intended } \\
\text { exposures }\end{array}$ & Missing data & $\begin{array}{c}\text { Outcome } \\
\text { measurement }\end{array}$ & $\begin{array}{c}\text { Selection of the } \\
\text { reported result }\end{array}$ \\
\hline Cahill, 2013 $\mathbf{2 0}^{1}$ & Moderate & Serious & Serious & Critical & Moderate & Moderate & Moderate \\
\hline Karatzi, 2016 & Moderate & Serious & Serious & Critical & Low & Moderate & Serious \\
\hline
\end{tabular}

iii A detailed description of the methodology used for assessing risk of bias is available on the NESR website: https://nesr.usda.gov/2020-dietary-guidelinesadvisory-committee-systematic-reviews and in Part C of the following reference: Dietary Guidelines Advisory Committee. 2020. Scientific Report of the 2020 Dietary Guidelines Advisory Committee: Advisory Report to the Secretary of Agriculture and the Secretary of Health and Human Services. U.S. Department of Agriculture, Agricultural Research Service, Washington, DC.

iv Possible ratings of low, moderate, serious, critical, or no information determined using the "Risk of Bias for Nutrition Observational Studies" tool (RoB-NObs) (Dietary Guidelines Advisory Committee. 2020. Scientific Report of the 2020 Dietary Guidelines Advisory Committee: Advisory Report to the Secretary of Agriculture and the Secretary of Health and Human Services. U.S. Department of Agriculture, Agricultural Research Service, Washington, DC.) 
The NESR team used its rigorous, protocol-driven methodology to support the 2020 Dietary Guidelines Advisory Committee in conducting this systematic review.

NESR's systematic review methodology involves:

- Developing a protocol,

- Searching for and selecting studies,

- Extracting data from and assessing the risk of bias of each included study,

- Synthesizing the evidence,

- Developing conclusion statements,

- Grading the evidence underlying the conclusion statements, and

- Recommending future research.

A detailed description of the methodology used in conducting this systematic review is available on the NESR website: https://nesr.usda.gov/2020-dietary-guidelines-advisory-committee-systematicreviews, and can be found in the 2020 Dietary Guidelines Advisory Committee Report, Part C: Methodology. ${ }^{\vee}$ This systematic review was peer reviewed by Federal scientists, and information about the peer review process can also be found in the Committee's Report, Part C. Methodology. Additional information about this systematic review, including a description of and rationale for any modifications made to the protocol can be found in the 2020 Dietary Guidelines Advisory Committee Report, Chapter 13. Frequency of Eating.

Below are details of the final protocol for the systematic review described herein, including the:

- Analytic framework

- Literature search and screening plan

- Literature search and screening results

\section{ANALYTIC FRAMEWORK}

The analytic framework (Figure 1) illustrates the overall scope of the systematic review, including the population, the interventions and/or exposures, comparators, and outcomes of interest. It also includes definitions of key terms and identifies key confounders considered in the systematic review. The inclusion and exclusion criteria that follow provide additional information about how parts of the analytic framework were defined and operationalized for the review.

v Dietary Guidelines Advisory Committee. 2020. Scientific Report of the 2020 Dietary Guidelines Advisory Committee: Advisory Report to the Secretary of Agriculture and the Secretary of Health and Human Services. U.S. Department of Agriculture, Agricultural Research Service, Washington, DC. 


\section{Figure 1: Analytic framework}

\section{Systematic review question: What is the relationship between the frequency of eating and risk of}

cardiovascular disease?

\begin{tabular}{|c|c|c|}
\hline Intervention/exposure vs Comparator & & Endpoint outcome(s) \\
\hline $\begin{array}{l}\begin{array}{l}\text { Frequency of eating: } \\
\begin{array}{l}\text { Number of daily eating } \\
\text { occasions }\end{array} \\
\begin{array}{l}\text { Population: Children and adolescents (ages } 2-18 \text { years } \\
\text { of daily eating }\end{array} \\
\text { old); adults (ages 19-64 years old); women during } \\
\text { pregnancy or lactation; older adults (ages } 65 \text { and older); } \\
\text { healthy and/or at risk of chronic disease }\end{array} \\
\end{array}$ & $\begin{array}{l}\text { Intermediate outcome(s) } \\
\text { - Total cholesterol (TC) } \\
\text { - } \text { LDL Cholesterol } \\
\text { TCL Cholesterol (including } \\
\text { - Triglycerides } \\
\text { - Blood pressure (systolic and } \\
\text { diastolic) } \\
\text { Population: Children and } \\
\text { adolescents (ages 2-18 years } \\
\text { old); adults (ages } 19-64 \text { years } \\
\text { old); women during pregnancy } \\
\text { or lactation; older adults (ages } \\
65 \text { and older) }\end{array}$ & $\begin{array}{l}\text { - Cardiovascular disease } \\
\text { (myocardial infarction, coronary } \\
\text { heart disease, coronary artery } \\
\text { disease, congestive heart failure, } \\
\text { peripheral artery disease) } \\
\text { - Stroke } \\
\text { - Venous thrombosis } \\
\text { - Cardiovascular disease-related } \\
\text { mortality } \\
\text { Population: Children and } \\
\text { adolescents (ages } 2-18 \text { years old); } \\
\text { adults (ages 19-64 years old); } \\
\text { women during pregnancy or } \\
\text { lactation; older adults (ages } 65 \text { and } \\
\text { older) }\end{array}$ \\
\hline
\end{tabular}

Key Confounders: Sex, Age, Race/ethnicity, Habitual eating frequency, Physical activity, Smoking, Anthropometry

Other factors to be considered: Socioeconomic status, Cultural practices, Diet energy density, Total energy intake, Energy state of the diet (restriction/surplus), Energy balance (total energy intake/total energy expenditure), Chrononutrition factors (time of day, (consistency of) habitual eating frequency, and duration between ingestive events and/or ingestive periods), Portion size, Macronutrient content, Location of eating occasion, Specialized diet including all-liquids diet, Dietary sodium and potassium, Dietary fat composition, Eating environment (who you eat with, work/schooVexercise schedule), Holiday eating (seasonal), Sleep schedule (shift work), Secondary eating, Dentition, Hydration status, Pregnancy status, Pubertal status, Menopausal status, Family history of cardiovascular disease, Medication and substance use, Biochemical changes

\section{Key definitions}

Eating occasion - ingestive event [preload, meals or snacks; food or beverage (energy yielding or non-energy yielding)]

Secondary eating- eating occasions that are not identified as the primary activity (e.g., screen time, eating while driving, reading)

\section{Legend}

The relationship of interest in the systematic review .......... Factors that may impact the relationship of interest in the systematic review 


\section{LITERATURE SEARCH AND SCREENING PLAN}

\section{Inclusion and exclusion criteria}

This table provides the inclusion and exclusion criteria for the systematic review. The inclusion and exclusion criteria are the set of characteristics used to determine which articles identified in the literature search were included in or excluded from the systematic review.

\section{Table 3. Inclusion and exclusion criteria}

\begin{tabular}{|c|c|c|}
\hline Category & Inclusion Criteria & Exclusion Criteria \\
\hline Study design & $\begin{array}{l}\text { - } \text { Randomized controlled trials } \\
\text { - Non-randomized controlled trials, including quasi- } \\
\text { experimental and controlled before and after } \\
\text { studies } \\
\text { - } \quad \text { Prospective cohort studies } \\
\text { - } \quad \text { Retrospective cohort studies } \\
\text { - } \quad \text { Nested case-control studies }\end{array}$ & $\begin{array}{l}\text { - Uncontrolled trials } \\
\text { - } \text { Case-control studies } \\
\text { - } \text { Cross-sectional studies } \\
\text { - Uncontrolled before-and-after studies } \\
\text { - } \quad \text { Narrative reviews } \\
\text { - } \quad \text { Systematic reviews } \\
\text { - } \quad \text { Meta-analyses }\end{array}$ \\
\hline $\begin{array}{l}\text { Intervention/ } \\
\text { exposure }\end{array}$ & $\begin{array}{l}\text { Frequency of eating: } \\
\text { - Number of daily eating occasions }\end{array}$ & $\begin{array}{l}\text { - Studies that only examine frequency of } \\
\text { intake of a single food, beverage or category } \\
\text { of foods or beverages (i.e. frequency of milk } \\
\text { consumption, frequency of seafood } \\
\text { consumption) } \\
\text { - Studies that do not have eating occasions } \\
\text { across the day }\end{array}$ \\
\hline Comparator & - Different number of daily eating occasions & - N/A \\
\hline Outcomes & $\begin{array}{l}\text { Intermediate Outcomes: } \\
\text { - } \quad \text { Total cholesterol (TC) } \\
\text { - } \text { LDL cholesterol } \\
\text { - } \quad \text { HDL cholesterol (including TC:HDL and LDL:HDL } \\
\text { ratios) } \\
\text { - Triglycerides } \\
\text { - Blood pressure (systolic and diastolic) } \\
\text { Endpoint Outcomes: } \\
\text { - Cardiovascular disease (myocardial infarction, } \\
\quad \text { coronary heart disease, coronary artery disease, } \\
\text { congestive heart failure, peripheral artery } \\
\text { - } \text { disease) } \\
\text { - Vtroke } \\
\text { - Cardiovascular disease-related mortality }\end{array}$ & \\
\hline
\end{tabular}




\begin{tabular}{|c|c|c|}
\hline Category & Inclusion Criteria & Exclusion Criteria \\
\hline $\begin{array}{l}\text { Date of } \\
\text { publication }\end{array}$ & - January 2000 - September 2019 & $\begin{array}{l}\text { - Articles published prior to or after January } \\
2000 \text { - September } 2019\end{array}$ \\
\hline $\begin{array}{l}\text { Publication } \\
\text { status }\end{array}$ & - Articles published in peer-reviewed journals & $\begin{array}{l}\text { - Articles that have not been peer-reviewed } \\
\text { and are not published in peer-reviewed } \\
\text { journals (e.g. unpublished data, manuscripts, } \\
\text { reports, abstracts, pre-prints, and conference } \\
\text { proceedings) }\end{array}$ \\
\hline $\begin{array}{l}\text { Language of } \\
\text { publication }\end{array}$ & - Articles published in English & $\begin{array}{l}\text { - Articles published in languages other than } \\
\text { English }\end{array}$ \\
\hline Country vi & $\begin{array}{l}\text { - Studies conducted in countries ranked as high or } \\
\text { very high human development }\end{array}$ & $\begin{array}{l}\text { - Studies conducted in countries ranked as } \\
\text { medium or lower human development }\end{array}$ \\
\hline $\begin{array}{l}\text { Study } \\
\text { participants }\end{array}$ & $\begin{array}{l}\text { - Human participants } \\
\text { - Males } \\
\text { - Females } \\
\text { - } \begin{array}{l}\text { Females during pregnancy and the post-partum } \\
\text { period }\end{array}\end{array}$ & $\begin{array}{l}\text { - Non-human participants (i.e., animals or in- } \\
\text { vitro models) }\end{array}$ \\
\hline $\begin{array}{l}\text { Age of study } \\
\text { participants }\end{array}$ & $\begin{array}{l}\text { - Age at intervention or exposure: } \\
\quad \bigcirc \text { Children and adolescents (2-18 years) } \\
\circ \text { Adults (19-64 years) } \\
\circ \text { Older adults (ages } 65 \text { and older) } \\
\text { - Age at outcome: } \\
\circ \text { Children and adolescents (2-18 years) } \\
\circ \text { Adults (19-64 years) } \\
\text { - Older adults (ages } 65 \text { and older) }\end{array}$ & $\begin{array}{l}\text { - Age at intervention or exposure: } \\
\qquad \quad \begin{array}{l}\text { Infants and toddlers (0-24 months } \\
\text { old) }\end{array} \\
\text { - Age at outcome: } \\
\circ \quad \begin{array}{l}\text { Infants and toddlers (0-24 months } \\
\text { old) }\end{array}\end{array}$ \\
\hline
\end{tabular}

vi In order to determine the inclusion exclusion criteria for country, the Human Development classification was used. This classification is based on the Human Development Index (HDI) ranking from the year the study intervention occurred or data were collected (UN Development Program. HDI 1990-2017 HDRO calculations based on data from UNDESA (2017a), UNESCO Institute for Statistics (2018), United Nations Statistics Division (2018b), World Bank (2018b), Barro and Lee (2016) and IMF (2018). Available from: http://hdr.undp.org/en/data). If the study did not report the year in which the intervention occurred or data were collected, the HDI classification for the year of publication was applied. HDI values are available from 1980, and then from 1990 to present. If a study was conducted prior to 1990, the HDI classification from 1990 was applied. If a study was conducted in 2018 or 2019, the most current HDI classification was applied. When a country was not included in the HDI ranking, the current country classification from the World Bank was used instead (The World Bank. World Bank country and lending groups. Available from: https://datahelpdesk.worldbank.org/knowledgebase/articles/906519-world- country-and-lending-groups) 


\begin{tabular}{lll}
\hline Category Inclusion Criteria & Exclusion Criteria
\end{tabular}

Health status of - Studies that enroll participants who are healthy study participants with obesity and/or at risk for chronic disease, including those

- Studies that enroll some participants diagnosed with a disease

- Studies that exclusively enroll participants with high blood pressure or high cholesterol and are evaluating cardiovascular disease endpoint outcomes (i.e., studies that aim to prevent cardiovascular disease in participants who are at high risk)

- Studies that enroll some participants with endpoint outcomes of cardiovascular disease
- Studies that exclusively enroll participants diagnosed with a disease, or hospitalized with an illness or injury. (For this criterion, studies that exclusively enroll participants with obesity will not be excluded).

- Studies that exclusively enroll participants with high blood pressure or high cholesterol and are evaluating blood pressure or cholesterol outcomes (i.e., studies that aim to treat participants who already have high blood pressure or high cholesterol)

- Studies that exclusively enroll participants with endpoint outcomes of cardiovascular disease (i.e., studies that aim to treat participants who have already been diagnosed with the endpoint outcomes of interest)

- Studies that exclusively enroll subjects post bariatric surgery

Eating
frequency data
collection for
intervention
studies

- Data collection for eating frequency that occurs on at least 2 occasions, including baseline and during or after the intervention.

- Each occasion encompasses a minimum of 3,24-hour periods or a questionnaire that covers at least 3 days addressing eating frequency.

- (e.g., 3, 24-h dietary recalls reporting ingestive events)

- (e.g., 1 eating frequency questionnaire documenting eating frequency for the past month)

\section{Eating frequency data collection for observational studies}

- Data collection for eating frequency that encompasses a minimum of 3, 24-hour periods

○ (e.g., 3, 24-h dietary recalls reporting each ingestive event)

- (e.g., 1 eating frequency questionnaire documenting eating frequency for the past month)

\begin{tabular}{|c|c|c|}
\hline $\begin{array}{l}\text { Size of study } \\
\text { groups for } \\
\text { intervention } \\
\text { studies }\end{array}$ & $\begin{array}{l}\text { - } 15 \text { or greater participants for studies using within- } \\
\text { subject analyses, or } \\
\text { - } 30 \text { or greater participants for studies using } \\
\text { between-subject analysis, or } \\
\text { - } \quad \text { A power calculation included }\end{array}$ & $\begin{array}{l}\text { - Fewer than } 15 \text { participants for studies using } \\
\text { within-subject analyses, or } \\
\text { - Fewer than } 30 \text { participants for studies using } \\
\text { between-subject analysis, or } \\
\text { - No power calculation reported }\end{array}$ \\
\hline
\end{tabular}

- Data collection for eating frequency that occurs on fewer than 2 occasions, and encompasses fewer than 3, 24-hour periods
- Data collection for eating frequency that encompasses fewer than 3, 24-hour periods 


\section{Electronic databases and search terms}

\section{PubMed}

- Provider: U.S. National Library of Medicine

- Date(s) searched: September 172019

- Date range searched: January 1, 2000-September 172019

- Search Terms:

\#1 - "frequency of eating" OR eating frequenc* OR "frequent eating" OR feeding frequenc* OR "Meals"[Mesh] OR meal frequenc* OR "meal timing" OR "meal time" OR mealtim* OR daily meal* OR dinnertim* OR dinner pattern* OR "night eating" OR evening meal* OR eating occasion* OR irregular eat* OR snack frequenc* OR snacking frequenc* OR snacking pattern* OR snacking behavior" OR "Fasting"[Mesh] OR "intermittent fasting" OR fasting diet* OR "alternate-day fasting" OR "meal skipping" OR "breakfast skipping" OR skipping breakfast* OR "Feeding Behavior"[Mesh:noexp] OR feeding behavior*[tiab] OR eating episode* OR eating pattern* OR eating habit* OR eating tim* OR "eating alone" OR time restricted feeding ${ }^{*}$ OR feeding pattern* OR meal profile* OR meal pattern* OR meal environment* OR chrononutrition OR intermittent energy restriction* OR intermittent diet*

\#2 - "Cardiovascular Diseases"[Mesh:noexp] OR cardiovascular disease*[tiab] OR coronary artery disease[tiab] OR heart disease*[tiab] OR "Heart Failure"[Mesh] OR heart failure[tiab] OR "Myocardial Infarction"[Mesh] OR myocardial infarction*[tiab] OR "Myocardial Ischemia"[Mesh] OR Myocardial Ischemia*[tiab] OR "Stroke"[Mesh] OR stroke[tiab] OR angina[tiab] OR heart attack[tiab] OR "Venous Thrombosis"[Mesh] OR venous thrombosis[tiab] OR hypertension[tiab] OR high blood pressure[tiab] OR "Lipids/blood"[Mesh] OR "Cholesterol, HDL"[Mesh] OR HDL cholesterol[tiab] OR "Cholesterol, LDL"[Mesh] OR LDL cholesterol[tiab] OR total cholesterol[tiab] OR "Triglycerides"[Mesh] OR triglycerides[tiab]

\#3 - "Diabetes Mellitus, Type 2"[Mesh] OR Type 2 diabetes[tiab] OR T2D[tiab] OR adult onset diabetes[tiab] OR "Prediabetic State"[Mesh] OR prediabet"[tiab] OR pre diabet* OR "Insulin Resistance"[Mesh] OR insulin resistance[tiab] OR "Glucose Intolerance"[Mesh] OR glucose intolerance[tiab] OR glucose tolerance[tiab] OR "Glycated Hemoglobin A"[Mesh] OR hemoglobin A1c[ti] OR "Hyperglycemia"[Mesh] OR "Hypoglycemia"[Mesh] OR ((impaired fasting[tiab] OR "Diabetes Mellitus"[Mesh:noexp]) AND (glucose[tiab] OR glycemi*[tiab] OR high blood sugar[tiab] OR low blood sugar[tiab]

\#4 - "Body Weights and Measures"[Mesh] OR "Body Weight"[Mesh] OR body weight[tiab] OR "Overnutrition"[Mesh:NoExp] OR overnutrition[tiab] OR body weight[tiab] OR weight status[tiab] OR obesity[tiab] OR obese[tiab] OR overweight[tiab] OR body mass index[tiab] OR BMI[tiab] OR underweight[tiab] OR wasting[tiab] OR healthy weight[tiab] OR "Body Composition"[Mesh] OR body composition[tiab] OR body fat[tiab] OR fat mass[tiab] OR fat free mass[tiab] OR "Adipose Tissue"[Mesh] OR "Adiposity"[Mesh] OR adipos*[tiab] OR anthropometry[tiab] OR anthropometric*[tiab] OR body height[tiab] OR stunting[tiab] OR stunted[tiab] OR "Growth Charts"[Mesh] OR growth chart*[tiab] OR waist circumference[tiab] OR head circumference[tiab] OR arm circumference[tiab] OR thigh circumference[tiab] OR neck circumference[tiab] OR "Gestational Weight Gain"[Mesh] OR "Weight Gain"[Mesh:NoExp] OR weight gain[tiab] OR "Body Size"[Mesh] OR "body size"[tiab] OR weight change[tiab] OR weight changes[tiab] OR "Weight Loss"[Mesh] OR weight loss*[tiab] OR weight reduc*[tiab] OR "Weight Reduction Programs"[Mesh] OR "Body-Weight Trajectory"[Mesh] OR weight maint* OR "Diet, Reducing"[Mesh] OR diet reduc*[tiab] OR weight cycling[tiab] OR weight 
decreas*[tiab] OR weight watch*[tiab] OR weight control*[tiab] OR weight retention[tiab] OR (weight[tiab] AND (reduction OR reduced OR reducing OR loss OR losses OR maintenanc* OR maintain*[tiab] OR decreas*[tiab] OR watch OR control*[tiab] OR change*[tiab] OR gain[tiab]))

\#5 - (\#2 OR \#3 OR \#4)

\#6 - (\#1 AND \#5)

\#7 - (\#1 AND \#5) NOT (“Animals"[Mesh] NOT (“Animals"[Mesh] AND “Humans"[Mesh])) NOT (editorial[ptyp] OR comment[ptyp] OR news[ptyp] OR letter[ptyp] OR review[ptyp] OR systematic review[ptyp] OR systematic review[ti] OR meta-analysis[ptyp] OR meta-analysis[ti] OR meta-analyses[ti] OR retracted publication[ptyp] OR retraction of publication[ptyp] OR retraction of publication[tiab] OR retraction notice[ti]) Filters: Publication date from 2000/01/01 to 2019/09/17; English

\section{Cochrane Central Register of Controlled Trials (CENTRAL)}

- Provider: John Wiley \& Sons

- Date(s) searched: September 17, 2019

- Date range searched: January 1, 2000-September 17, 2019

- Search Terms:

\#1 - [mh Meals] OR [mh Fasting] OR [mh ^"Feeding Behavior"]

\#2 - "frequency of eating" OR "eating frequenc" OR "frequent eating" OR "feeding frequenc" OR "meal frequenc" OR "meal timing" OR "meal time" OR mealtim* OR "daily meal" OR dinnertim* OR "dinner pattern*" OR "night eating" OR "evening meal"” OR "eating occasion"” OR "irregular eat" $\mathrm{OR}$ "snack frequenc" OR "snacking frequenc" OR "snacking pattern" OR "snacking behavior" OR "intermittent fasting" OR "fasting diet" OR "alternate-day fasting" OR "meal skipping" OR "breakfast skipping" OR "skipping breakfast" OR "feeding behavior*" OR "eating episode" OR "eating pattern" OR "eating habit" OR "eating tim" OR "eating alone" OR "time restricted feeding" OR "feeding pattern*" OR "meal profile*" OR "meal pattern*" OR "meal environment" "OR chrono-nutrition OR "intermittent energy restriction" OR "intermittent diet*"

\#3 - \#1 OR \#2

\#4 - [mh ^"Cardiovascular Diseases"] OR [mh "Heart Failure"] OR [mh "Myocardial Infarction"] OR [mh "Myocardial Ischemia"] OR [mh Stroke] OR [mh "Venous Thrombosis"] OR [mh Lipids/BL] OR [mh "Cholesterol, HDL"] OR [mh "Cholesterol, LDL"] OR [mh Triglycerides]

\#5 - ("cardiovascular disease *" OR "coronary artery disease" OR "heart disease" OR "heart failure" OR "myocardial infarction*" OR "myocardial ischemia*" OR stroke OR angina OR "heart attack" OR "venous thrombosis" OR "hypertension" OR "high blood pressure" OR "HDL cholesterol" OR "LDL cholesterol" OR "total cholesterol" OR triglycerides):ti,ab,kw

\#6 - \#4 OR \#5

\#7 - [mh "Diabetes Mellitus, Type 2"] OR [mh "Prediabetic State"] OR [mh "Insulin Resistance"] OR [mh "Glucose Intolerance"] OR [mh "Glycated Hemoglobin A"] OR [mh Hyperglycemia] OR [mh Hypoglycemia]

\#8 - (“Type 2 diabetes" OR T2D OR "adult onset diabetes" OR prediabet* OR pre diabet* OR 
"insulin resistance" OR "glucose intolerance" OR "glucose tolerance" OR "hemoglobin A1c"):ti,ab,kw

\#9 - (("impaired fasting" OR [mh ^"Diabetes Mellitus"]) AND (glucose OR glycemi OR "high blood sugar" OR "low blood sugar"))

\#10 - \#7 OR \#8 OR \#9

\#11 - [mh "Body Weights and Measures"] OR [mh "Body Weight"] OR [mh ^Overnutrition] OR [mh "Body Composition"] OR [mh "Adipose Tissue"] OR [mh Adiposity] OR [mh "Growth Charts"] OR [mh "Gestational Weight Gain"] OR [mh ^"Weight Gain"] OR [mh "Body Size"] OR [mh "Weight Loss"] OR [mh "Weight Reduction Programs"] OR [mh "Body-Weight Trajectory"] OR [mh "Diet, Reducing"]

\#12 - "body weight" OR overnutrition OR "body weight" OR "weight status" OR obesity OR obese OR overweight OR "body mass index" OR BMI OR underweight OR wasting OR "healthy weight" OR "body composition" OR "body fat" OR "fat mass" OR "fat free mass" OR

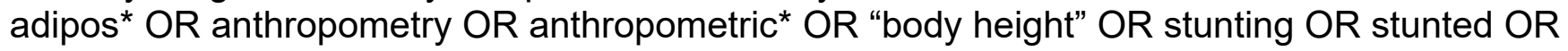
"growth chart" OR "waist circumference" OR "head circumference" OR "arm circumference" OR "thigh circumference" OR "neck circumference" OR "weight gain" OR "body size" OR "weight change" OR "weight changes" OR "weight loss"” OR "weight reduc" OR "weight maint" OR "diet reduc" OR "weight cycling" OR "weight decreas" OR "weight watch" OR "weight control*" OR "weight retention"

\#13 - ((weight NEAR/4 (reduction OR reduced OR reducing OR loss OR losses OR maintenanc* OR maintain* OR decreas* OR watch OR control* OR change ${ }^{*}$ OR gain))):ti,ab,kw

\#14 - \#11 OR \#12 OR \#13

\#15 - \#6 OR \#10 OR \#14

\#16 - \#3 AND \#15" with Publication Year from 2000 to 2019, in Trials (Word variations have been searched)

\section{Embase}

- Provider: Elsevier

- Date(s) searched: September 17, 2019

- Date range searched: January 1, 2000-September 17, 2019

- Search Terms:

\#1 - 'meal'/exp OR 'fasting'/exp OR 'feeding behavior'/de

\#2 - 'frequency of eating':ab,ti OR 'eating frequenc' :ab,ti OR 'frequent eating':ab,ti OR 'feeding frequenc':ab,ti OR 'meal frequenc':ab,ti OR 'meal timing':ab,ti OR 'meal time':ab,ti OR mealtim":ab,ti OR 'daily meal'”:ab,ti OR dinnertim*:ab,ti OR 'dinner pattern"':ab,ti OR 'night eating':ab,ti OR 'evening meal' :ab,ti OR 'eating occasion"':ab,ti OR 'irregular eat"':ab,ti OR 'snack frequenc' behavior"':ab,ti OR 'intermittent fasting':ab,ti OR 'fasting diet"':ab,ti OR 'alternate-day fasting':ab,ti OR 'meal skipping':ab,ti OR 'breakfast skipping':ab,ti OR 'skipping breakfast'”:ab,ti OR 'feeding behavior*':ab,ti OR 'eating episode "':ab,ti OR 'eating pattern'”:ab,ti OR 'eating habit': ab,ti OR 'eating tim':ab,ti OR 'eating alone':ab,ti OR 'time restricted feeding'”:ab,ti OR 
'feeding pattern'”:ab,ti OR 'meal profile*':ab,ti OR 'meal pattern*':ab,ti OR 'meal environment' :ab,ti OR 'chrono nutrition':ab,ti OR 'intermittent energy restriction'”:ab,ti OR 'intermittent diet"':ab,ti

\section{\#3 - \#1 OR \#2}

\#4 - 'cardiovascular disease'/de OR 'heart failure'/exp OR 'heart infarction'/exp OR 'heart muscle ischemia'/exp OR 'cerebrovascular accident'/exp OR 'vein thrombosis'/exp OR 'high density lipoprotein cholesterol'/de OR 'low density lipoprotein cholesterol'/de OR 'triacylglycerol'/exp

\#5 - 'cardiovascular disease*':ab,ti OR 'coronary artery disease':ab,ti OR 'heart disease':ab,ti OR 'heart failure':ab,ti OR 'myocardial infarction*':ab,ti OR 'myocardial ischemia*':ab,ti OR stroke:ab,ti OR angina:ab,ti OR 'heart attack':ab,ti OR 'venous thrombosis':ab,ti OR 'hypertension':ab,ti OR 'high blood pressure':ab,ti OR 'hdl cholesterol':ab,ti OR 'Idl cholesterol':ab,ti OR 'total cholesterol':ab,ti OR triglycerides:ab,ti

\section{\#6 - \#4 OR \#5}

\#7 - 'non insulin dependent diabetes mellitus'/exp OR 'impaired glucose tolerance'/exp OR 'insulin resistance'/de OR 'glucose intolerance'/de OR 'glycosylated hemoglobin'/exp OR 'hyperglycemia'/de OR 'hypoglycemia'/exp

\#8 - 'type 2 diabetes':ab,ti OR t2d:ab,ti OR 'adult onset diabetes':ab,ti OR prediabet':ab,ti OR 'pre diabet': ab,ti OR 'insulin resistance':ab,ti OR 'glucose intolerance':ab,ti OR 'glucose tolerance':ab,ti OR 'hemoglobin a1c':ab,ti

\#9 - (('impaired fasting' OR 'diabetes mellitus]') NEAR/4 (glucose OR glycemi* OR 'high blood sugar' OR 'low blood sugar')):ab,ti

\section{\#10 - \#7 OR \#8 OR \#9}

\#11 - 'weight, mass and size'/exp OR 'body weight'/exp OR 'overnutrition'/de OR 'body composition'/exp OR 'adipose tissue'/exp OR 'growth chart'/de OR 'gestational weight gain'/de OR 'body weight gain'/de OR 'body size'/de OR 'body weight loss'/exp OR 'weight loss program'/de OR 'weight trajectory (body weight)'/de OR 'low calorie diet'/exp

\#12 - overnutrition:ab,ti OR 'body weight':ab,ti OR 'weight status':ab,ti OR obesity:ab,ti OR obese:ab,ti OR overweight:ab,ti OR 'body mass index':ab,ti OR bmi:ab,ti OR underweight:ab,ti OR wasting:ab,ti OR 'healthy weight':ab,ti OR 'body composition':ab,ti OR 'body fat':ab,ti OR 'fat mass':ab,ti OR 'fat free mass':ab,ti OR adipos":ab,ti OR anthropometry:ab,ti OR anthropometric*:ab,ti OR 'body height':ab,ti OR stunting:ab,ti OR stunted:ab,ti OR 'growth chart*':ab,ti OR 'waist circumference':ab,ti OR 'head circumference':ab,ti OR 'arm circumference': $a b, t i$ OR 'thigh circumference': ab,ti OR 'neck circumference':ab,ti OR 'weight gain':ab,ti OR 'body size':ab,ti OR 'weight change':ab,ti OR 'weight changes':ab,ti OR 'weight loss"':ab,ti OR 'weight reduc':ab,ti OR 'weight maint"':ab,ti OR 'diet reduc':ab,ti OR 'weight cycling':ab,ti OR 'weight decreas"':ab,ti OR 'weight watch"':ab,ti OR 'weight control' :ab,ti OR 'weight retention':ab,ti

\#13 - (weight NEAR/4 (reduction OR reduced OR reducing OR loss OR losses OR maintenanc* OR maintain* OR decreas* OR watch OR control* OR change* OR gain)):ab,ti

\#14 - \#11 OR \#12 OR \#13

\#15 - \#6 OR \#10 OR \#14 
\#16 - \#3 AND \#15

\#17 - \#3 AND \#15 AND ([article]/lim OR [article in press]/lim) AND [humans]/lim AND [english]/lim AND [2000-2019]/py NOT ([conference abstract]/lim OR [conference review]/lim OR [conference paper]/lim OR [editorial]/lim OR [erratum]/lim OR [letter]/lim OR [note]/lim OR [review]/lim OR [systematic review]/lim OR [meta analysis]/lim)

\section{CINAHL Plus (Cumulative Index to Nursing and Allied Health Literature)}

- Provider: EBSCOhost

- Date(s) searched: September 24, 2019

- Date range searched: January 1, 2000-September 24, 2019

- Search Terms:

\#S1 - (MH "Meals+") OR (MH "Fasting") OR (MH "Eating Behavior")

\#S2 - "frequency of eating" OR "eating frequenc*" OR "frequent eating" OR "feeding frequenc" OR "meal frequenc" OR "meal timing" OR "meal time" OR mealtim* OR "daily meal" OR dinnertim* OR "dinner pattern" OR "night eating" OR "evening meal"” OR "eating occasion"” OR "irregular eat" $O R$ "snack frequenc" OR "snacking frequenc" OR "snacking pattern" OR "snacking behavior" OR "intermittent fasting" OR "fasting diet*" OR "alternate-day fasting" OR "meal skipping" OR "breakfast skipping" OR "skipping breakfast"” OR "feeding behavior" OR "eating episode*" OR "eating pattern" OR "eating habit" OR "eating tim" OR "time restricted feeding" OR "feeding pattern" OR "meal profile*" OR "meal pattern" OR "meal environment" " OR chrono-nutrition OR "intermittent energy restriction*" OR "intermittent diet"”

\#S3 - S1 OR S2

\#S4 - (MH "Cardiovascular Diseases") OR (MH "Heart Failure+") OR (MH "Myocardial Infarction+") OR (MH "Myocardial Ischemia+") OR (MH "Stroke+") OR (MH "Venous Thrombosis+") OR (MH “Lipids/BL") OR (MH “Lipoproteins, HDL Cholesterol”) OR (MH "Lipoproteins, LDL Cholesterol") OR (MH “Triglycerides")

\#S5 - "cardiovascular disease*" OR "coronary artery disease" OR "heart disease*"OR "heart failure" OR "myocardial infarction*" OR "myocardial Ischemia*" OR stroke OR angina OR "heart attack" OR "venous thrombosis" OR hypertension OR "high blood pressure" OR "HDL cholesterol" OR "LDL cholesterol" OR "total cholesterol" OR triglycerides

\#S6 - S4 OR S5

\#S7 - (MH "Diabetes Mellitus, Type 2") OR (MH "Prediabetic State") OR (MH "Insulin Resistance+") OR (MH "Glucose Intolerance") OR (MH "Hemoglobin A, Glycosylated") OR (MH "Hyperglycemia+") OR (MH "Hypoglycemia+")

\#S8 - ("Type 2 diabetes" OR T2D OR "adult onset diabetes" OR prediabet* OR pre diabet* OR "insulin resistance" OR "glucose intolerance" OR "glucose tolerance" OR "hemoglobin A1c")

\#S9 - ((MH "Diabetes Mellitus" OR "impaired fasting") N4 (glucose OR glycemi* OR "high blood sugar" OR "low blood sugar"))

\#S10 - S7 OR S8 OR S9

\#S11 - (MH "Body Weights and Measures+") OR (MH "Body Weight+") OR (MH "Body 
Composition+") OR (MH "Adipose Tissue") OR (MH "Gestational Weight Gain") OR (MH "Weight Gain+") OR (MH "Body Size") OR (MH "Weight Loss+") OR (MH "Weight Reduction Programs") OR (MH "Body Weight Changes") OR (MH "Diet, Reducing")

\#S12 - "body weight" OR overnutrition OR "body weight" OR "weight status" OR obesity OR obese OR overweight OR "body mass index" OR BMI OR underweight OR wasting OR "healthy weight" OR "body composition" OR "body fat" OR "fat mass" OR "fat free mass" OR adipos* OR anthropometry OR anthropometric* OR "body height" OR stunting OR stunted OR "growth chart" OR "waist circumference" OR "head circumference" OR "arm circumference" OR "thigh circumference" OR "neck circumference" OR "weight gain" OR "body size" OR "weight change" OR "weight changes" OR "weight loss" OR "weight reduc" OR "weight maint" OR "diet reduc*" OR "weight cycling" OR "weight decreas*” OR "weight watch*" OR "weight control*" OR "weight retention"

\#S13 - ((weight N4 (reduction OR reduced OR reducing OR loss OR losses OR maintenanc* OR maintain* OR decreas* OR watch OR control* OR change* OR gain))

\#S14 - S11 OR S12 OR S13

\#S15 - S6 OR S10 OR S14

\#S16 - (S3 AND S15) NOT (MH "Literature Review" OR MH "Meta Analysis" OR MH "Systematic Review" OR MH "News" OR MH "Retracted Publication" OR MH "Retraction of Publication) Publication Year: 2000-2019; Peer Reviewed; English Language; Human

\section{LITERATURE SEARCH AND SCREENING RESULTS}

The flow chart (Figure 2) below illustrates the literature search and screening results for articles examining the systematic review question. The literature search was conducted to identify articles for 5 different, but related systematic reviews on frequency of eating and growth, size, and body composition, cardiovascular disease, type 2 diabetes, post-partum weight loss, and gestational weight gain. The results of the electronic database searches, after removal of duplicates, were screened independently by two NESR analysts using a stepwise process by reviewing titles, abstracts, and full-texts to determine which articles met the inclusion criteria. Refer to Table 4 for the rationale for exclusion for each excluded full-text article. A manual search was done to find articles that were not identified when searching the electronic databases; all manually identified articles were also screened to determine whether they meet criteria for inclusion.

The literature search and screening results from multiple questions on frequency of eating were combined for efficiency because of topical overlap. The searches were designed to comprehensively identify relevant literature in all examined systematic reviews questions to avoid screening the same results multiple times. 
Figure 2: Flow chart of literature search and screening results

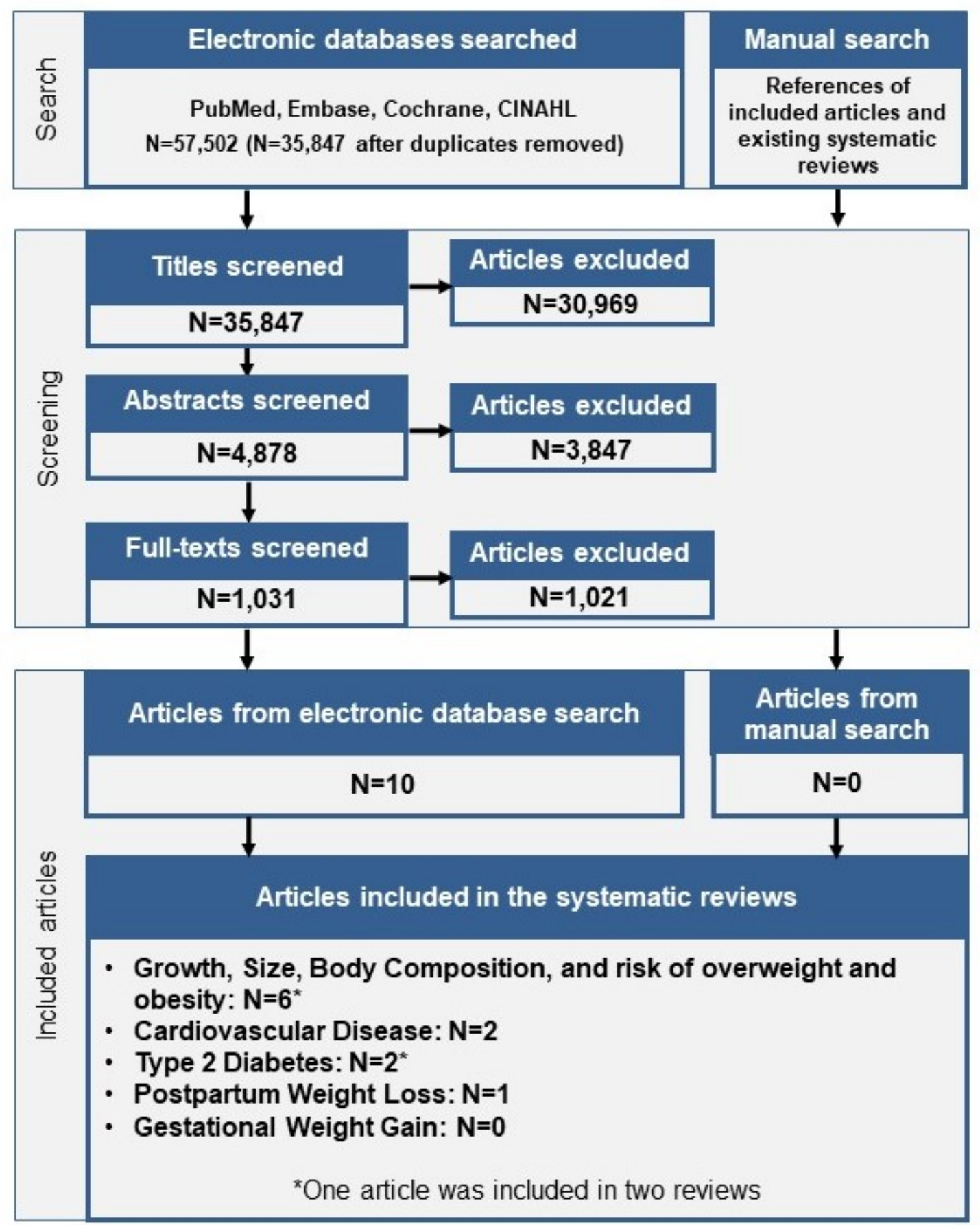




\section{Excluded articles}

The table below lists the articles excluded after full-text screening, and includes a column for the rationale for study exclusion based on the criteria in Table 3. At least one reason for exclusion is provided for each article, though this may not reflect all possible reasons for exclusion. Information about articles excluded after title and abstract screening is available upon request.

\section{Table 4. Articles excluded after full text screening with rationale for exclusion}

\section{Citation}

$1 \quad$ A little at a time: eating and exercising in bits and pieces. Harv Mens Health Watch. 2006. 11:6-7 https://www.ncbi.nlm.nih.gov/pubmed/17153760

2 Abdullah NF, Teo PS, Foo LH. Ethnic Differences in the Food Intake Patterns and Its Associated Factors of Adolescents in Kelantan, Malaysia. Nutrients. 2016;8(9). https://www.ncbi.nlm.nih.gov/pubmed/27626444.

3 Abendroth A, Michalsen A, Ludtke R, Ruffer A, Musial F, Dobos GJ, Langhorst J. Changes of Intestinal Microflora in Patients with Rheumatoid Arthritis during Fasting or a Mediterranean Diet. Forsch Komplementmed. 2010. 17:30713 https://www.ncbi.nlm.nih.gov/pubmed/21196744

$4 \quad$ Adachi Y, Sato C, Yamatsu K, Ito S, Adachi K, Yamagami T. A randomized controlled trial on the long-term effects of a 1-month behavioral weight control program assisted by computer tailored advice. Behav Res Ther. 2007. 45:459-70 https://www.ncbi.nlm.nih.gov/pubmed/16713991

$5 \quad$ Adawi M, Damiani G, Bragazzi NL, Bridgewood C, Pacifico A, Conic RRZ, et al. The Impact of Intermittent Fasting (Ramadan Fasting) on Psoriatic Arthritis Disease Activity, Enthesitis, and Dactylitis: A Multicentre Study. Nutrients. 2019;11(3). https://www.ncbi.nlm.nih.gov/pubmed/30871045.

6 Adegboye AR, Rossner S, Neovius M, Lourenco PM, Linne Y. Relationships between prenatal smoking cessation, gestational weight gain and maternal lifestyle characteristics. Women Birth. 2010. 23:29-35 https://www.ncbi.nlm.nih.gov/pubmed/19586807

7 Affenito SG, Thompson D, Dorazio A, Albertson AM, Loew A, Holschuh NM. Ready-to-eat cereal consumption and the School Breakfast Program: relationship to nutrient intake and weight. J Sch Health. 2013. 83:28-35 https://www.ncbi.nlm.nih.gov/pubmed/23253288

8 Breakfast consumption by African-American and white adolescent girls correlates positively with calcium and fiber intake and negatively with body mass index. J Am Diet Assoc. 2005. 105:938-45 https://www.ncbi.nlm.nih.gov/pubmed/15942545

\section{Rationale}

Publication Status

\section{Study Design}

Publication Status

Intervention/Exposure, Daily Eating Occasions Not Reported

Daily Eating Occasions Not Reported, Eating Frequency Data Collection

Intervention/Exposure

Study Design

Daily Eating Occasions Not Reported 
10 Afrasiabi A, Hassanzadeh S, Sattarivand R, Mahboob S. Effects of Ramadan fasting on serum lipid profiles on 2 hyperlipidemic groups with or without diet pattern. Saudi Med J. 2003. 24:23-6 https://www.ncbi.nlm.nih.gov/pubmed/12590268

11 Afrasiabi A, Hassanzadeh S, Sattarivand R, Nouri M, Mahbood S. Effects of low fat and low calorie diet on plasma lipid levels in the fasting month of Ramadan. Saudi Med J. 2003. 24:184-8 https://www.ncbi.nlm.nih.gov/pubmed/12682685

12 Agras WS, Hammer LD, McNicholas F, Kraemer HC. Risk factors for childhood overweight: a prospective study from birth to 9.5 years. J Pediatr. 2004. 145:20-5 https://www.ncbi.nlm.nih.gov/pubmed/15238901

13 Aila Gustafsson S, Edlund B, Kjellin L, Norring C. Risk and protective factors for disturbed eating in adolescent girls

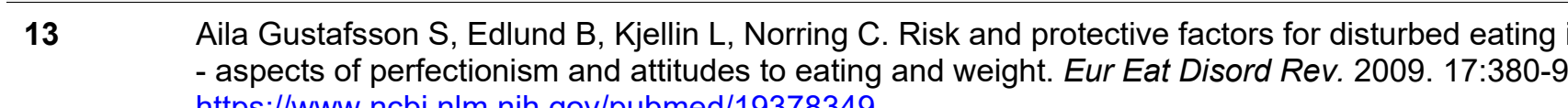

Aila Gustafsson S, Edlund B, Kjellin L, Norring C. Risk and protective factors for disturbed eating
- aspects of perfectionism and attitudes to eating and weight. Eur Eat Disord Rev. 2009. 17:380-9
https://www.ncbi.nlm.nih.gov/pubmed/19378349 Ajabnoor GM, Bahijri S, Borai A, Abdulkhaliq AA, Al-Aama JY, Chrousos GP. Health impact of fasting in Saudi Arabia during Ramadan: association with disturbed circadian rhythm and metabolic and sleeping patterns. PLoS

\section{One. 2014. 9:e96500 https://www.ncbi.nlm.nih.gov/pubmed/24810091 \\ 14}

Ajabnoor GM, Bahijri S, Shaik NA, Borai A, Alamoudi AA, Al-Aama JY, Chrousos GP. Ramadan fasting in Saudi
Arabia is associated with altered expression of CLOCK, DUSP and IL-1alpha genes, as well as changes in
cardiometabolic risk factors. PLoS One. 2017. 12:e0174342 https://www.ncbi.nlm.nih.gov/pubmed/28384165

Ajabnoor GM, Bahijri S, Shaik NA, Borai A, Alamoudi AA, Al-Aama JY, Chrousos GP. Ramadan fasting in Saud
Arabia is associated with altered expression of CLOCK, DUSP and IL-1alpha genes, as well as changes in
cardiometabolic risk factors. PLoS One. 2017. 12:e0174342 https://www.ncbi.nlm.nih.gov/pubmed/28384165

Ajabnoor GM, Bahijri S, Shaik NA, Borai A, Alamoudi AA, Al-Aama JY, Chrousos GP. Ramadan fasting in Saud
Arabia is associated with altered expression of CLOCK, DUSP and IL-1alpha genes, as well as changes in
cardiometabolic risk factors. PLoS One. 2017. 12:e0174342 https://www.ncbi.nlm.nih.gov/pubmed/28384165

16 Akanji AO, Mojiminiyi OA, Abdella N. Beneficial changes in serum apo A-1 and its ratio to apo B and HDL in stable hyperlipidaemic subjects after Ramadan fasting in Kuwait. Eur J Clin Nutr. 2000. 54:508-13 https://www.ncbi.nlm.nih.gov/pubmed/10878654

17 Akhan G, Kutluhan S, Koyuncuoglu HR. Is there any change of stroke incidence during Ramadan?. Acta Neurol Scand. 2000. 101:259-61 https://www.ncbi.nlm.nih.gov/pubmed/10770523

18 Akimoto-Gunther L, Hubler M, Santos M, Carolino I, Sonoo N, Botti B, Mota D, Takahachi G. Effects of re-education in eating habits and physical activity on the lipid profile of obese teenagers. Clin Chem Lab Med. 2002. 40:460-2 https://www.ncbi.nlm.nih.gov/pubmed/12113288

19 Aksungar FB, Eren A, Ure S, Teskin O, Ates G. Effects of intermittent fasting on serum lipid levels, coagulation status and plasma homocysteine levels. Ann Nutr Metab. 2005. 49:77-82 https://www.ncbi.nlm.nih.gov/pubmed/15802901

Daily Eating Occasions Not Reported, Country

Intervention/Exposure, Daily Eating Occasions Not Reported

Intervention/Exposure, Daily Eating Occasions Not Reported

Intervention/Exposure, Daily Eating Occasions Not Reported

Daily Eating Occasions Not Reported

Daily Eating Occasions Not Reported

Intervention/Exposure, Daily Eating Occasions Not Reported

Intervention/Exposure, Daily Eating Occasions Not Reported

Study Design,

Intervention/Exposure, Daily Eating Occasions Not Reported

Intervention/Exposure, Daily Eating Occasions Not Reported 
20 Aksungar FB, Sarikaya M, Coskun A, Serteser M, Unsal I. Comparison of Intermittent Fasting Versus Caloric Restriction in Obese Subjects: A Two Year Follow-Up. J Nutr Health Aging. 2017. 21:681-685

Study Design, Size of Study https://www.ncbi.nlm.nih.gov/pubmed/28537332

21 Aksungar FB, Topkaya AE, Akyildiz M. Interleukin-6, C-reactive protein and biochemical parameters during prolonged intermittent fasting. Ann Nutr Metab. 2007. 51:88-95 https://www.ncbi.nlm.nih.gov/pubmed/17374948

Daily Eating Occasions Not prolonged intermittent fasting. Ann Nutr Metab. 2007. 51:88-95 https.//www.ncbi.nlm.nih.gov/pubmed/17374948

Reported 22 Aktas MF, Mahler A, Hamm M, Perger G, Simon F, Westenhofer J, Luft FC, Boschmann M. Lifesty
Muslim patients with metabolic syndrome-a feasibility study. Eur J Clin Nutr. 2019. 73:805-808
https://www.ncbi.nlm.nih.gov/pubmed/30538299

23 Al Assaad RG, Bachir R, El Sayed MJ. Impact of Ramadan on emergency department visits and on medical emergencies. Eur J Emerg Med. 2018. 25:440-444 https://www.ncbi.nlm.nih.gov/pubmed/28704270

Intervention/Exposure Al Suwaidi J, Bener A, Hajar HA, Numan MT. Does hospitalization for congestive heart failure occur more frequently

24 Al Suwaidi J, Bener A, Hajar HA, Numan MT. Does hospitalization for congestive hear https://www.ncbi.nlm.nih.gov/pubmed/15262036

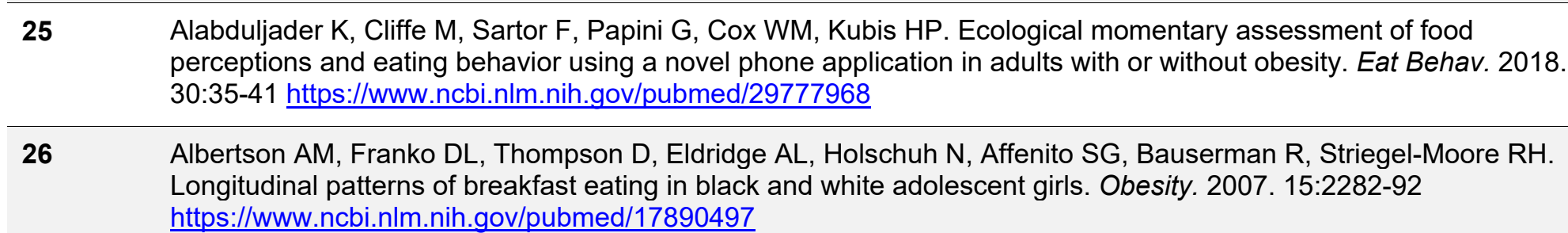

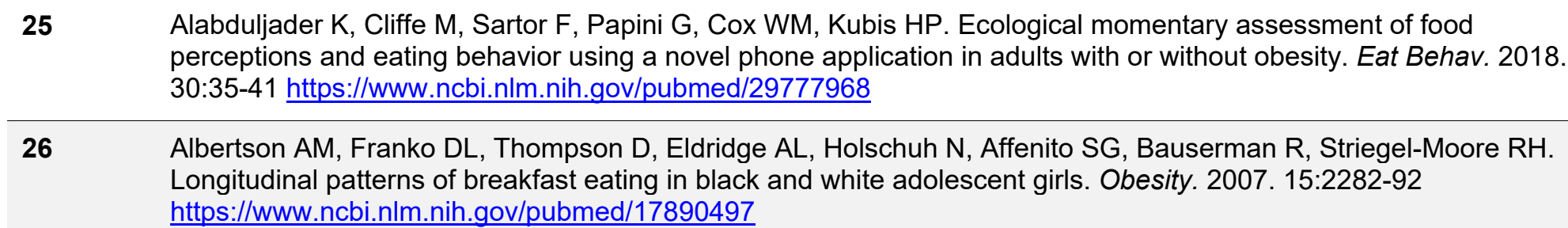

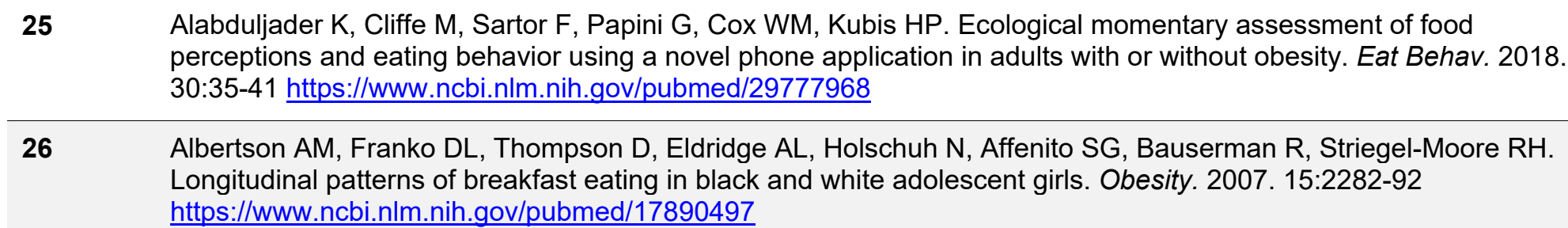

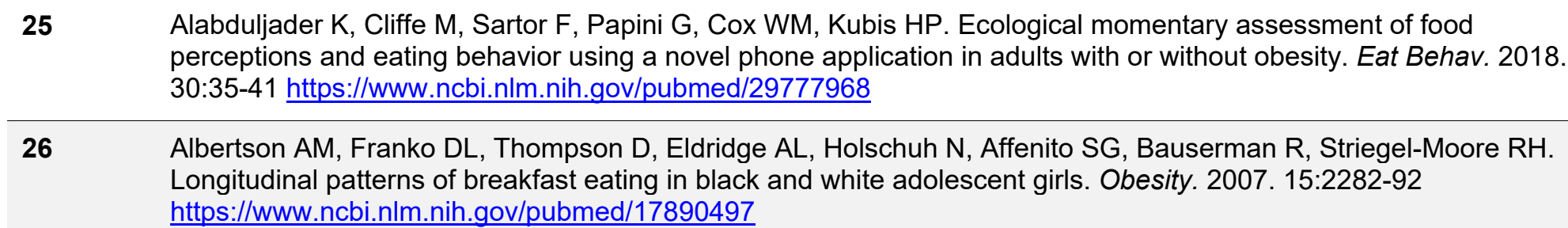

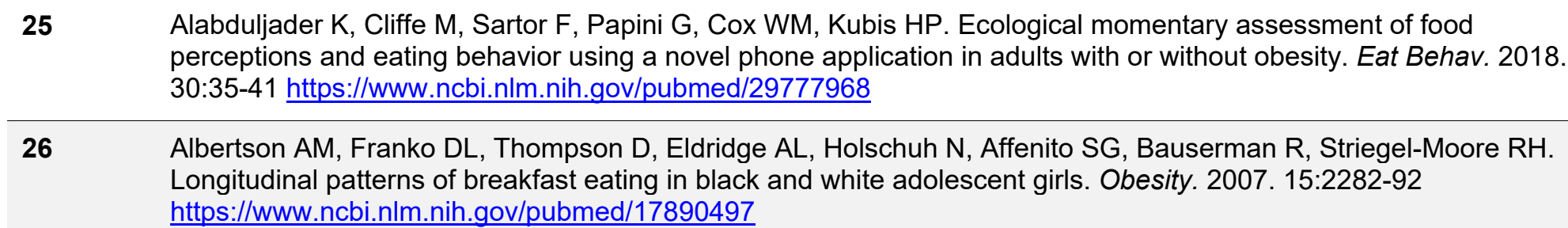

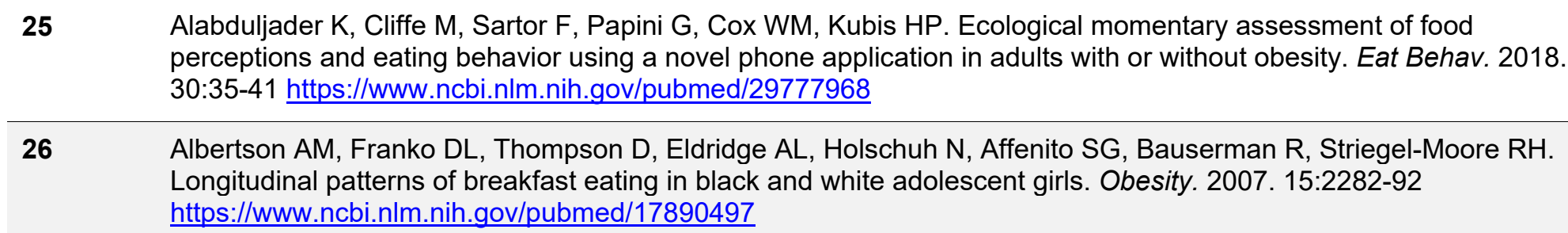
Aldhoon-Hainerova I, Hainer V, Zamrazilova $\mathrm{H}$. Impact of dietary intake, lifestyle and biochemical factors on metabolic health in obese adolescents. Nutr Metab Cardiovasc Dis. 2017. 27:703-710

27 Aldhoon-Hainerova I, Hainer V, Zamrazilova H. Imp
metabolic health in obese adolescents. Nutr Metab
https://www.ncbi.nlm.nih.gov/pubmed/28693964

28 Alencar MK, Beam JR, McCormick JJ, White AC, Salgado RM, Kravitz LR, Mermier CM, Gibson AL, Conn CA, Kolkmeyer D, Ferraro RT, Kerksick CM. Increased meal frequency attenuates fat-free mass losses and some markers of health status with a portion-controlled weight loss diet. Nutr Res. 2015. 35:375-83 https://www.ncbi.nlm.nih.gov/pubmed/25862614 Eating Disorder Examination. Eat Behav. 2016. 21:7-10 https://www.ncbi.nlm.nih.gov/pubmed/26722817

Daily Eating Occasions Not Reported

Intervention/Exposure, Daily Eating Occasions Not Reported

Intervention/Exposure

Daily Eating Occasions Not Reported

Study Design

Eating Frequency Data Collection

Study Design, Outcome 
31 Al-Hazzaa HM, Musaiger AO, Abahussain NA, Al-Sobayel HI, Alsulaiman NA, Tayyem RF, et al. Physical activity patterns and eating habits of adolescents living in major Arab cities. The Arab Teens Lifestyle Study. Saudi medical

Reported journal. 2010;31(2):210-1. https://www.ncbi.nlm.nih.gov/pubmed/20174744.

\begin{tabular}{|c|c|}
\hline 32 & $\begin{array}{l}\text { Al-Hourani HM, Atoum MF. Body composition, nutrient intake and physical activity patterns in young women during } \\
\text { Ramadan. Singapore Med J. 2007. 48:906-10 https://www.ncbi.nlm.nih.gov/pubmed/17909674 }\end{array}$ \\
\hline 33 & $\begin{array}{l}\text { Alhussain M, Macdonald IA, Taylor MA. Deleterious effects of irregular meal pattern on dietary thermogenesis in } \\
\text { obese women. Proceedings of the nutrition society. 2016. 75:E6 }\end{array}$ \\
\hline
\end{tabular}

34 Alhussain M, Taylor MA, MacDonald IA. Influence of the constancy of daily meal pattern on postprandial energy expenditure in healthy weight women. Proceedings of the nutrition society. 2015. 74: OCE1
35 Alhussain MH, Macdonald IA, Taylor MA. Irregular meal-pattern effects on energy expenditure, metabolism, and appetite regulation: a randomized controlled trial in healthy normal-weight women. Am J Clin Nutr. 2016. 104:21-32 https://www.ncbi.nlm.nih.gov/pubmed/27305952

36 Ali Z, Abizari AR. Ramadan fasting alters food patterns, dietary diversity and body weight among Ghanaian adolescents. Nutr J. 2018. 17:75 https://www.ncbi.nlm.nih.gov/pubmed/30098591

37 Alken J, Petriczko E, Marcus C. Effect of fasting on young adults who have symptoms of hypoglycemia in the absence of frequent meals. Eur J Clin Nutr. 2008. 62:721-6 https://www.ncbi.nlm.nih.gov/pubmed/17522614

$38 \quad$ Alleman RJ, Harvey IC, Farney TM, Bloomer RJ. Both a traditional and modified Daniel Fast improve the cardiometabolic profile in men and women. Lipids Health Dis. 2013. 12:114 https://www.ncbi.nlm.nih.gov/pubmed/23889755

39 Allerton DM, Campbell MD, Gonzalez JT, West DJ, Stevenson EJ. The effect of breakfast macronutrient content or skipping breakfast on 24h glucose profiles. Diabetic medicine. 2015. 32:55 (Suppl.1) Physiol Behav. 2013. 110-111:169-78 https://www.ncbi.nlm.nih.gov/pubmed/23333553

\section{Study Design}

Daily Eating Occasions Not Reported

Study Design, Publication Status

Study Design, Publication Status

Eating Frequency Data

Collection

Daily Eating Occasions Not Reported, Country

Intervention/Exposure, Daily Eating Occasions Not Reported, Comparator

Comparator, Size of Study Groups

Publication Status

Daily Eating Occasions Not

Reported 
41 Allirot X, Seyssel K, Saulais L, Roth H, Charrie A, Drai J, Goudable J, Blond E, Disse E, Laville M. Effects of a breakfast spread out over time on the food intake at lunch and the hormonal responses in obese men. Physiol Behav. 2014. 127:37-44 https://www.ncbi.nlm.nih.gov/pubmed/24472321

42 Allison KC, Ahima RS, O'Reardon JP, Dinges DF, Sharma V, Cummings DE, Heo M, Martino NS, Stunkard AJ.

Daily Eating Occasions Not Reported

Neuroendocrine profiles associated with energy intake, sleep, and stress in the night eating syndrome. J Clin
Endocrinol Metab. 2005. 90:6214-7 https://www.ncbi.nlm.nih.gov/pubmed/16131578

Neuroendocrine profiles associated with energy intake, sleep, and stress in the night
Endocrinol Metab. 2005. 90:6214-7 https://www.ncbi.nlm.nih.gov/pubmed/16131578

43 Almeneessier AS, Bahammam AS, Sharif MM, Bahammam SA, Nashwan SZ, Pandi Perumal SR, Cardinali DP, Alzoghaibi M. The influence of intermittent fasting on the circadian pattern of melatonin while controlling for caloric intake, energy expenditure, light exposure, and sleep schedules: A preliminary report. Ann Thorac Med. 2017. 12:183-190 https://www.ncbi.nlm.nih.gov/pubmed/28808490

$44 \quad$ Almoosawi S, Prynne CJ, Hardy R, Stephen AM. Diurnal eating rhythms: association with long-term development of diabetes in the 1946 British birth cohort. Nutr Metab Cardiovasc Dis. 2013. 23:1025-30 https://www.ncbi.nlm.nih.gov/pubmed/23541169

$45 \quad$ Almoosawi S, Prynne CJ, Hardy R, Stephen AM. Time-of-day and nutrient composition of eating occasions: prospective association with the metabolic syndrome in the 1946 British birth cohort. Int J Obes (Lond). 2013. 37:725-31 https://www.ncbi.nlm.nih.gov/pubmed/22777542

46 Aloui A, Chaouachi A, Chtourou H, Wong del P, Haddad M, Chamari K, Souissi N. Effects of Ramadan on the diurnal variations of repeated-sprint performances. Int J Sports Physiol Perform. 2013. 8:254-62 https://www.ncbi.nlm.nih.gov/pubmed/22952200

47 Al-Ozairi E, Al Kandari J, AlHaqqan D, AlHarbi O, Masters Y, Syed AA. Obesity surgery and Ramadan: a prospective analysis of nutritional intake, hunger and satiety and adaptive behaviours during fasting. Obes Surg. 2015. 25:523-9 https://www.ncbi.nlm.nih.gov/pubmed/25595382

$48 \quad$ Al-Shafei Al. Ramadan fasting ameliorates arterial pulse pressure and lipid profile, and alleviates oxidative stress in hypertensive patients. Blood Press. 2014. 23:160-7 https://www.ncbi.nlm.nih.gov/pubmed/24059637

49 Alsharairi NA, Somerset SM. Skipping breakfast in early childhood and its associations with maternal and child BMI: a study of 2-5-year-old Australian children. Eur J Clin Nutr. 2016. 70:450-5 https://www.ncbi.nlm.nih.gov/pubmed/26508462

50
Alsubheen SA, Ismail M, Baker A, Blair J, Adebayo A, Kelly L, Chandurkar V, Cheema S, Joanisse DR, Basset FA. The effects of diurnal Ramadan fasting on energy expenditure and substrate oxidation in healthy men. Br J Nutr. 2017. 118:1023-1030 https://www.ncbi.nlm.nih.gov/pubmed/29198194
Study Design,

Intervention/Exposure, Daily Eating Occasions Not Reported

Daily Eating Occasions Not Reported, Outcome, Size of Study Groups

Intervention/Exposure

Intervention/Exposure, Daily Eating Occasions Not Reported

Daily Eating Occasions Not Reported, Outcome

Health Status

Intervention/Exposure, Daily Eating Occasions Not Reported

Daily Eating Occasions Not Reported

Daily Eating Occasions Not Reported 
$51 \quad$ Alwasel SH, Abotalib Z, Aljarallah JS, Osmond C, Alkharaz SM, Alhazza IM, Harrath A, Thornburg K, Barker DJ. Sex differences in birth size and intergenerational effects of intrauterine exposure to Ramadan in Saudi Arabia. Am

Daily Eating Occasions Not J Hum Biol. 2011. 23:651-4 https://www.ncbi.nlm.nih.gov/pubmed/21630372

52 Alwasel SH, Harrath A, Aljarallah JS, Abotalib Z, Osmond C, Al Omar SY, Khaled I, Barker DJ. Intergenerational effects of in utero exposure to Ramadan in Tunisia. Am J Hum Biol. 2013. 25:341-3

Reported, Outcome https://www.ncbi.nlm.nih.gov/pubmed/23436278

$53 \quad$ Alwattar AY, Thyfault JP, Leidy HJ. The effect of breakfast type and frequency of consumption on glycemic

Daily Eating Occasions Not Reported, Outcome response in overweight/obese late adolescent girls. Eur J Clin Nutr. 2015. 69:885-90 https://www.ncbi.nlm.nih.gov/pubmed/25711955

54 Amigo-Vazquez I, Busto-Zapico R, Errasti-Perez JM, Pena-Suarez E. Skipping breakfast, sedentarism and overweight in children. Psychol Health Med. 2016. 21:819-26 https://www.ncbi.nlm.nih.gov/pubmed/26728102

53

\section{5}

55 Ammerman A, Leung MM, Cavallo D. Addressing disparities in the obesity epidemic. N C Med J. 2006. 67:301-4
https://www.ncbi.nlm.nih.gov/pubmed/17066662

56 Amodio D, D'Amico M, Meret L, Gaizo A, Laviano A. Time restricted feeding (TRF) enhances weight loss efficiency in dietary restricted women with metabolic syndrome. Clinical nutrition. 2016. 35:S39-

$57 \quad$ Amosa T, Rush E, Plank L. Frequency of eating occasions reported by young New Zealand Polynesian and European women. Pac Health Dialog. 2001. 8:59-65 https://www.ncbi.nlm.nih.gov/pubmed/12017838

$58 \quad$ Andersen AE, Klinger D. The overeater self and the healthy self within. Eating Disorders. 2002. 10:87-91

59 Andersen GS, Stunkard AJ, Sorensen TI, Petersen L, Heitmann BL. Night eating and weight change in middle-aged men and women. Int J Obes Relat Metab Disord. 2004. 28:1338-43 https://www.ncbi.nlm.nih.gov/pubmed/15278102

60 Anderson YC, Wynter LE, Butler MS, Grant CC, Stewart JM, Cave TL, Wild CE, Derraik JG, Cutfield WS, Hofman PL. Dietary Intake and Eating Behaviours of Obese New Zealand Children and Adolescents Enrolled in a Community-Based Intervention Programme. PLoS One. 2016. 11:e0166996 https://www.ncbi.nlm.nih.gov/pubmed/27880804

61 Andersson I, Lennernas M, Rossner S. Meal pattern and risk factor evaluation in one-year completers of a weight reduction program for obese men - the 'Gustaf' study. J Intern Med. 2000. 247:30-8 https://www.ncbi.nlm.nih.gov/pubmed/10672128 Androutsos O, Moschonis G, Mavrogianni C, Roma-Giannikou E, Chrousos GP, Kanaka-Gantenbein C, Manios Y. Identification of lifestyle patterns, including sleep deprivation, associated with insulin resistance in children: the Healthy Growth Study. Eur J Clin Nutr. 2014. 68:344-9 https://www.ncbi.nlm.nih.gov/pubmed/24424081

Intervention/Exposure, Daily Eating Occasions Not Reported

Study Design, Daily Eating Occasions Not Reported

Intervention/Exposure

Study Design, Publication Status

Study Design

Study Design

Intervention/Exposure, Daily Eating Occasions Not Reported

Intervention/Exposure, Daily Eating Occasions Not Reported

\section{Outcome}

Study Design 
63 Anton SD, Lee SA, Donahoo WT, McLaren C, Manini T, Leeuwenburgh C, et al. The Effects of Time Restricted Feeding on Overweight, Older Adults: A Pilot Study. Nutrients. 2019;11(7). https://www.ncbi.nlm.nih.gov/pubmed/31262054.

64 Antoni R, Johnston K, Collins A, Robertson M. The comparative effects of intermittent versus continuous energy restriction on postprandial glucose and lipid metabolism following 5\% weight-loss. Obesity facts. 2017. 10:S209

Daily Eating Occasions Not

Reported

Antoni R, Johnston KL, Collins A, Robertson MD. Investigation into the acute effects of intermittent energy restriction

Publication Status

Antoni R, Johnston KL, Collins A, Robertson MD. Investigation into the acute effects of inte
on postprandial substrate metabolism. Proceedings of the nutrition society. 2016. 75:E29

66 Antoni R, Johnston KL, Collins AL, Robertson MD. Acute effects of intermittent energy restriction on energy

compensation: a pilot study. Obesity facts. 2015. 8:76

67 Antoni R, Johnston KL, Collins AL, Robertson MD. Intermittent v. continuous energy restriction: differential effects on postprandial glucose and lipid metabolism following matched weight loss in overweight/obese participants. Br J Nutr. 2018. 119:507-516 https://www.ncbi.nlm.nih.gov/pubmed/29508693

68 Antoni R, Johnston KL, Collins AL, Robertson MD. Investigation into the acute effects of total and partial energy restriction on postprandial metabolism among overweight/obese participants. Br J Nutr. 2016. 115:951-9 https://www.ncbi.nlm.nih.gov/pubmed/26819200

69 Antoni R, Johnston KL, Collins AL, Robertson MD. The comparative effects of intermittent versus continuous energy restriction on postprandial glucose-lipid metabolism following $5 \%$ weight-loss: interim analysis of an ongoing study. Proceedings of the nutrition society. 2016. 75:E105

70 Aparicio A, Rodriguez-Rodriguez E, Aranceta-Bartrina J, Gil A, Gonzalez-Gross M, Serra-Majem L, Varela-Moreiras G, Ortega RM. Differences in meal patterns and timing with regard to central obesity in the ANIBES ('Anthropometric data, macronutrients and micronutrients intake, practice of physical activity, socioeconomic data and lifestyles in Spain') Study - CORRIGENDUM. Public Health Nutr. 2018. 21:1588 https://www.ncbi.nlm.nih.gov/pubmed/29506590

71 Appleton KM, Baker S. Distraction, not hunger, is associated with lower mood and lower perceived work performance on fast compared to non-fast days during intermittent fasting. $J$ Health Psychol. 2015. 20:702-11 https://www.ncbi.nlm.nih.gov/pubmed/26032787
Arciero PJ, Ormsbee MJ, Gentile CL, Nindl BC, Brestoff JR, Ruby M. Increased protein intake and meal frequency reduces abdominal fat during energy balance and energy deficit. Obesity (Silver Spring). 2013. 21:1357-66 https://www.ncbi.nlm.nih.gov/pubmed/23703835
Publication Status

Daily Eating Occasions Not

Reported, Size of Study Groups

Intervention/Exposure, Daily Eating Occasions Not Reported

Publication Status

Publication Status

Intervention/Exposure, Daily Eating Occasions Not Reported, Outcome

Size of Study Groups 
73 Arguin H, Dionne IJ, Senechal M, Bouchard DR, Carpentier AC, Ardilouze JL, Tremblay A, Leblanc C, Brochu M. Short- and long-term effects of continuous versus intermittent restrictive diet approaches on body composition and the metabolic profile in overweight and obese postmenopausal women: a pilot study. Menopause. 2012. 19:870-6

Daily Eating Occasions Not https://www.ncbi.nlm.nih.gov/pubmed/22735163

$74 \quad$ Aronoff NJ, Geliebter A, Zammit G. Gender and body mass index as related to the night-eating syndrome in obese outpatients. J Am Diet Assoc. 2001. 101:102-4 https://www.ncbi.nlm.nih.gov/pubmed/11209573

Reported

Intervention/Exposure, Daily Eating Occasions Not Reported

75 Asarnow LD, Greer SM, Walker MP, Harvey AG. The Impact of Sleep Improvement on Food Choices in Adolescents Intervention/Exposure With Late Bedtimes. J Adolesc Health. 2017. 60:570-576 https://www.ncbi.nlm.nih.gov/pubmed/28111011

76 Asci O, Rathfisch G. Effect of lifestyle interventions of pregnant women on their dietary habits, lifestyle behaviors, and weight gain: a randomized controlled trial. J Health Popul Nutr. 2016. 35:7 https://www.ncbi.nlm.nih.gov/pubmed/26911204

77 Ask AS, Hernes S, Aarek I, Johannessen G, Haugen M. Changes in dietary pattern in 15 year old adolescents following a 4 month dietary intervention with school breakfast--a pilot study. Nutr J. 2006;5:33. https://www.ncbi.nlm.nih.gov/pubmed/17150115.

78 Ask AS, Hernes S, Aarek I, Johannessen G, Haugen M. Changes in dietary pattern in 15 year old adolescents following a 4 month dietary intervention with school breakfast-a pilot study. Nutr J. 2006. 5:33 https://www.ncbi.nlm.nih.gov/pubmed/17150115

79 Askari VR, Alavinezhad A, Boskabady MH. The impact of "Ramadan fasting period" on total and differential white blood cells, haematological indices, inflammatory biomarker, respiratory symptoms and pulmonary function tests of healthy and asthmatic patients. Allergologia et Immunopathologia. 2016;44(4):359-67. https://www.ncbi.nlm.nih.gov/pubmed/27040808.

80 Assadi M, Akrami A, Beikzadeh F, Seyedabadi M, Nabipour I, Larijani B, Afarid M, Seidali E. Impact of Ramadan fasting on intraocular pressure, visual acuity and refractive errors. Singapore Med J. 2011. 52:263-6 https://www.ncbi.nlm.nih.gov/pubmed/21552787

81 Astbury NM, Taylor MA, Macdonald IA. Breakfast consumption affects appetite, energy intake, and the metabolic and endocrine responses to foods consumed later in the day in male habitual breakfast eaters. J Nutr. 2011. 141:1381-9 https://www.ncbi.nlm.nih.gov/pubmed/21562233

Daily Eating Occasions Not Reported

Intervention/Exposure, Daily Eating Occasions Not Reported

Daily Eating Occasions Not Reported

Daily Eating Occasions Not Reported

Daily Eating Occasions Not Reported, Outcome

Daily Eating Occasions Not Reported, Outcome

Study Design 

intervention?. Obesity (silver spring, $m d$.). 2011. 19:S61-S62 https://www.cochranelibrary.com/central/doi/10.1002/central/CN-01005798/full

\section{Bachman JL, Phelan S, Wing RR, Raynor HA. Eating frequency is higher in weight loss maintainers and normal-} weight individuals than in overweight individuals. J Am Diet Assoc. 2011. 111:1730-4 https://www.ncbi.nlm.nih.gov/pubmed/22027056

85 BaHammam A, Alrajeh M, Albabtain M, Bahammam S, Sharif M. Circadian pattern of sleep, energy expenditure, and body temperature of young healthy men during the intermittent fasting of Ramadan. Appetite. 2010;54(2):426-9. https://www.ncbi.nlm.nih.gov/pubmed/20100529.

\section{Bahammam AS, Almushailhi K, Pandi-Perumal SR, Sharif MM. Intermittent fasting during Ramadan: does it affect} sleep?. J Sleep Res. 2014. 23:35-43 https://www.ncbi.nlm.nih.gov/pubmed/23937329

87 Bahammam AS, Nashwan S, Hammad O, Sharif MM, Pandi-Perumal SR. Objective assessment of drowsiness and reaction time during intermittent Ramadan fasting in young men: a case-crossover study. Behav Brain Funct. 2013. 9:32 https://www.ncbi.nlm.nih.gov/pubmed/23937904

88 BaHammam AS, Pandi-Perumal SR, Alzoghaibi MA. The effect of Ramadan intermittent fasting on lipid peroxidation in healthy young men while controlling for diet and sleep: A pilot study. Ann Thorac Med. 2016. 11:43-8 https://www.ncbi.nlm.nih.gov/pubmed/26933456

89 Bahijri S, Borai A, Ajabnoor G, Abdul Khaliq A, AIQassas I, Al-Shehri D, Chrousos G. Relative metabolic stability, but disrupted circadian cortisol secretion during the fasting month of Ramadan. PLoS One. 2013. 8:e60917 https://www.ncbi.nlm.nih.gov/pubmed/23637777

90 Bak AM, Vendelbo MH, Christensen B, Viggers R, Bibby BM, Rungby J, Jorgensen JOL, Moller N, Jessen N. Prolonged fasting-induced metabolic signatures in human skeletal muscle of lean and obese men. PLoS One. 2018. 13:e0200817 https://www.ncbi.nlm.nih.gov/pubmed/30183740

91 Bakan AA, Yildiz S, Alkan A, Yetis H, Kurtcan S, Ilhan MM. Microstructural effects of Ramadan fasting on the brain: a diffusion tensor imaging study. Diagn Interv Radiol. 2015. 21:256-61 https://www.ncbi.nlm.nih.gov/pubmed/25835077

92 Balieiro LC, Rossato LT, Waterhouse J, Paim SL, Mota MC, Crispim CA. Nutritional status and eating habits of bus drivers during the day and night. Chronobiol Int. 2014. 31:1123-9 https://www.ncbi.nlm.nih.gov/pubmed/25231504

Daily Eating Occasions Not Reported

Daily Eating Occasions Not Reported

Intervention/Exposure, Daily Eating Occasions Not Reported

Daily Eating Occasions Not Reported

Intervention/Exposure, Daily Eating Occasions Not Reported

Intervention/Exposure, Daily Eating Occasions Not Reported

Intervention/Exposure, Daily Eating Occasions Not Reported, Outcome

\section{Study Design}

Study Design 
94 Barkia A, Mohamed K, Smaoui M, Zouari N, Hammami M, Nasri M. Change of diet, plasma lipids, lipoproteins, and fatty acids during Ramadan: a controversial association of the considered Ramadan model with atherosclerosis risk. J Health Popul Nutr. 2011. 29:486-93 https://www.ncbi.nlm.nih.gov/pubmed/22106754

95 Barnosky A, Kroeger CM, Trepanowski JF, Klempel MC, Bhutani S, Hoddy KK, Gabel K, Shapses SA, Varady KA. Effect of alternate day fasting on markers of bone metabolism: An exploratory analysis of a 6-month randomized controlled trial. Nutr Healthy Aging. 2017. 4:255-263 https://www.ncbi.nlm.nih.gov/pubmed/29276795

96 Baron KG, Reid KJ, Kern AS, Zee PC. Role of sleep timing in caloric intake and BMI. Obesity (Silver Spring). 2011. 19:1374-81 https://www.ncbi.nlm.nih.gov/pubmed/21527892

97 Baron KG, Reid KJ, Kim T, Van Horn L, Attarian H, Wolfe L, Siddique J, Santostasi G, Zee PC. Circadian timing and alignment in healthy adults: associations with BMI, body fat, caloric intake and physical activity. Int $J$ Obes (Lond). 2017. 41:203-209 https://www.ncbi.nlm.nih.gov/pubmed/27795550

\section{8} Basilakis A, Kiprouli K, Mantzouranis S, Konstantinidis T, Dionisopoulou M, Hackl JM, Balogh D. Nutritional study in Greek-Orthodox monasteries - Effect of a 40-day religious fasting. Aktuelle Ernahrungsmedizin. 2002. 27:250-255

99 Bataineh MF, Al-Nawaiseh AM, Abu Altaieb MH, Bellar DM, Hindawi OS, Judge LW. Impact of carbohydrate mouth rinsing on time to exhaustion during Ramadan: A randomized controlled trial in Jordanian men. Eur J Sport Sci. 2018. 18:357-366 https://www.ncbi.nlm.nih.gov/pubmed/29364063

100 Batista-Jorge GC, Barcala-Jorge AS, Oliveira Dias AF, Silveira MF, de Farias Lelis D, Oliveira Andrade JM, Claro RM, de Paula AM, Guimaraes AL, Ferreira AV, Santos SH. Nutritional Status Associated to Skipping Breakfast in Brazilian Health Service Patients. Ann Nutr Metab. 2016. 69:31-40 https://www.ncbi.nlm.nih.gov/pubmed/27434543

101 Baum Jl, Gaines BL, Kubas GC, Mitchell CF, Russell SL. Educational nutrition messaging at breakfast reduces snack intake and influences snack preferences in adult men and women. Appetite. 2017. 117:67-73 https://www.ncbi.nlm.nih.gov/pubmed/28627401

102 Baxter SD, Hardin JW, Guinn CH, Royer JA, Mackelprang AJ, Devlin CM. Children's body mass index, participation in school meals, and observed energy intake at school meals. International journal of behavioral nutrition and physical activity. 2010;7. https://www.ncbi.nlm.nih.gov/pubmed/20334667. Beebe D, Chang JJ, Kress K, Mattfeldt-Beman M. Diet quality and sleep quality among day and night shift nurses. $J$ Nurs Manag. 2017. 25:549-557 https://www.ncbi.nlm.nih.gov/pubmed/28695685

Beer A-M, Ismar LE, Wessely DK, Pötschke T, Weidner B, Wiebelitz KR. Retrospective Long-Term Comparison of Naturopathic Fasting Therapy and Weight Reduction Diet in Overweight Patients. Evidence-based Complementary \& Alternative Medicine (eCAM). 2014;2014:1-8. https://www.ncbi.nlm.nih.gov/pubmed/25126098.
Daily Eating Occasions Not Reported

Daily Eating Occasions Not Reported, Size of Study Groups

Study Design, Daily Eating Occasions Not Reported

Intervention/Exposure

\section{Study Design,}

Intervention/Exposure, Daily Eating Occasions Not Reported

Intervention/Exposure

\section{Study Design}

Intervention/Exposure, Daily Eating Occasions Not Reported

Intervention/Exposure, Daily Eating Occasions Not Reported

Study Design, Comparator, Outcome

Intervention/Exposure, Daily Eating Occasions Not Reported 

26 https://www.ncbi.nlm.nih.gov/pubmed/18059404

$106 \quad$ Bekersky I, Dressler D, Mekki Q. Effect of time of meal consumption on bioavailability of a single oral 5 mg tacrolimus dose. J Clin Pharmacol. 2001. 41:289-97 https://www.ncbi.nlm.nih.gov/pubmed/11269569

107 Bellisle F, Dalix AM, Mennen L, Galan P, Hercberg S, de Castro JM, Gausseres N. Contribution of snacks and meals in the diet of French adults: a diet-diary study. Physiol Behav. 2003. 79:183-9 https://www.ncbi.nlm.nih.gov/pubmed/12834789

108 Benchekroun MT, Moussamih S. Effects of Ramadan fasting on cardiovascular diseases. Saudi Med J. 2004. 25:1520-1 https://www.ncbi.nlm.nih.gov/pubmed/15494846

109 Bener A, Hamad A, Fares A, Al-Sayed HM, Al-Suwaidi J. Is there any effect of Ramadan fasting on stroke incidence?. Singapore Med J. 2006. 47:404-8 https://www.ncbi.nlm.nih.gov/pubmed/16645691

Outcome

Berg C, Lappas G, Wolk A, Strandhagen E, Toren K, Rosengren A, Thelle D, Lissner L. Eating pa

Berg C, Lappas G, Wolk A, Strandhagen E, Toren K, Rosengren A, Thelle D,
size associated with obesity in a Swedish population. Appetite. 2009. 52:21-6 https://www.ncbi.nlm.nih.gov/pubmed/18694791

111 Berge JM, Wall M, Larson N, Loth KA, Neumark-Sztainer D. Family functioning: associations with weight status, eating behaviors, and physical activity in adolescents. J Adolesc Health. 2013. 52:351-7 https://www.ncbi.nlm.nih.gov/pubmed/23299010

112 Berkey CS, Rockett HR, Gillman MW, Field AE, Colditz GA. Longitudinal study of skipping breakfast and weight change in adolescents. Int J Obes Relat Metab Disord. 2003. 27:1258-66 https://www.ncbi.nlm.nih.gov/pubmed/14513075

113 Bernardo LM, Stein H, Flavin PM. The ABCDiet program: evaluation of project outcomes. School Nurse News. 2010. 27:16-9 https://www.ncbi.nlm.nih.gov/pubmed/20945675

114 Bernice Dodor. The Impact of Religiosity on Health Behaviors and Obesity among African Americans. Journal of Human Behavior in the Social Environment. 2012. 22:451-462

115 Berteus Forslund $\mathrm{H}$, Klingstrom S, Hagberg $\mathrm{H}$, Londahl M, Torgerson JS, Lindroos AK. Should snacks be recommended in obesity treatment? A 1-year randomized clinical trial. Eur J Clin Nutr. 2008. 62:1308-17 https://www.ncbi.nlm.nih.gov/pubmed/17700649

\section{Study Design}

Intervention/Exposure, Daily Eating Occasions Not Reported

Study Design

Study Design

Daily Eating Occasions Not Reported

Study Design, Publication Status

Intervention/Exposure

Eating Frequency Data

Collection 
116 Bertz F, Sparud-Lundin C, Winkvist A. Transformative Lifestyle Change: key to sustainable weight loss among women in a post-partum diet and exercise intervention. Matern Child Nutr. 2015. 11:631-45 https://www.ncbi.nlm.nih.gov/pubmed/24750689

117 Bes-Rastrollo M, Sanchez-Villegas A, Basterra-Gortari FJ, Nunez-Cordoba JM, Toledo E, Serrano-Martinez M. Prospective study of self-reported usual snacking and weight gain in a Mediterranean cohort: the SUN project. Clin Nutr. 2010. 29:323-30 https://www.ncbi.nlm.nih.gov/pubmed/19748710

118 Betoko A, Lioret S, Heude B, Hankard R, Carles S, Forhan A, Regnault N, Botton J, Charles MA, de Lauzon-Guillain B. Influence of infant feeding patterns over the first year of life on growth from birth to 5 years. Pediatr Obes. 2017. 12 Suppl 1:94-101 https://www.ncbi.nlm.nih.gov/pubmed/28299906

119 Bhattarai MD, Singh DL. Excessive weight gain after pregnancy in urban areas: one important area to prevent diabetes. Nepal Med Coll J. 2005. 7:87-9 https://www.ncbi.nlm.nih.gov/pubmed/16519070

120 Bhutani S, Klempel MC, Berger RA, Varady KA. Improvements in coronary heart disease risk indicators by alternate-day fasting involve adipose tissue modulations. Obesity (Silver Spring). 2010. 18:2152-9 https://www.ncbi.nlm.nih.gov/pubmed/20300080

121 Bhutani S, Klempel MC, Kroeger CM, Aggour E, Calvo Y, Trepanowski JF, Hoddy KK, Varady KA. Effect of exercising while fasting on eating behaviors and food intake. J Int Soc Sports Nutr. 2013. 10:50 https://www.ncbi.nlm.nih.gov/pubmed/24176020

122 Bhutani S, Klempel MC, Kroeger CM, Trepanowski JF, Phillips SA, Norkeviciute E, Varady KA. Alternate day fasting with or without exercise: effects on endothelial function and adipokines in obese humans. E-spen journal. 2013. 8:e205-e209

123 Bhutani S, Klempel MC, Kroeger CM, Trepanowski JF, Varady KA. Alternate day fasting and endurance exercise combine to reduce body weight and favorably alter plasma lipids in obese humans. Obesity (Silver Spring). 2013. 21:1370-9 https://www.ncbi.nlm.nih.gov/pubmed/23408502

124 Bhutani S, Klempel MC, Kroeger CM, Varady KA. Combining alternate day fasting with exercise improves plasma lipids and Idl particle size in obese humans. Clinical nutrition, supplement. 2012. 7:10-11

Daily Eating Occasions Not Reported

Age: Intervention/Exposure Intervention/Exposure, Country

Daily Eating Occasions Not Reported

Intervention/Exposure, Daily Eating Occasions Not Reported

Daily Eating Occasions Not Reported

Daily Eating Occasions Not Reported

Study Design, Publication Status

125 Bhutani S, Klempel MC, Kroeger CM, Varady KA. OP024 Combining alternate day fasting with exercise improves plasma lipids and Idl particle size in obese humans. Clinical Nutrition Supplements. 2012. 7:10-11 Bhutani S, Varady KA, Klempel MC, Kroeger CM. Alternate day fasting combined with exercise: an effective treatment for weight loss and cardio-protection in obese humans. FASEB journal. 2012;26. 
127 Bjornshave A, Holst JJ, Hermansen K. A pre-meal of whey proteins induces differential effects on glucose and lipid metabolism in subjects with the metabolic syndrome: a randomised cross-over trial. Eur J Nutr. 2019. 58:755-764

Intervention/Exposure https://www.ncbi.nlm.nih.gov/pubmed/29626232

128 Bloomgarden ZT. Prevention of cardiovascular disease. Diabetes care. 2007. 30:423-431 https://www.cochranelibrary.com/central/doi/10.1002/central/CN-01721552/full

Study Design,

Intervention/Exposure

129 Bo S, De Carli L, Venco E, Fanzola I, Maiandi M, De Michieli F, Durazzo M, Beccuti G, Cavallo-Perin P, Ghigo E, Ganzit GP. Impact of snacking pattern on overweight and obesity risk in a cohort of 11- to 13-year-old adolescents. J Pediatr Gastroenterol Nutr. 2014. 59:465-71 https://www.ncbi.nlm.nih.gov/pubmed/24897170

130 Bo S, Fadda M, Castiglione A, Ciccone G, De Francesco A, Fedele D, Guggino A, Parasiliti Caprino M, Ferrara S, Vezio Boggio M, Mengozzi G, Ghigo E, Maccario M, Broglio F. Is the timing of caloric intake associated with variation in diet-induced thermogenesis and in the metabolic pattern? A randomized cross-over study. Int $J$ Obes (Lond). 2015. 39:1689-95 https://www.ncbi.nlm.nih.gov/pubmed/26219416

131 Bocca G, Kuitert MWB, Sauer PJJ, Corpeleijn E. Effect of a multidisciplinary treatment program on eating behavior in overweight and obese preschool children. J Pediatr Endocrinol Metab. 2018. 31:507-513 https://www.ncbi.nlm.nih.gov/pubmed/29652666

132 Boonchoo W, Takemi Y, Hayashi F, Koiwai K, Ogata H. Dietary intake and weight status of urban Thai preadolescents in the context of food environment. Prev Med Rep. 2017. 8:153-157 https://www.ncbi.nlm.nih.gov/pubmed/29881667

133 Booth DA, Blair AJ, Lewis VJ, Baek SH. Patterns of eating and movement that best maintain reduction in overweight. Appetite. 2004. 43:277-83 https://www.ncbi.nlm.nih.gov/pubmed/15527930

\section{Study Design}

Intervention/Exposure, Daily Eating Occasions Not Reported, Comparator

Intervention/Exposure, Outcome

\section{Study Design}

Borzecki A, Zolkowska D, Sieklucka-Dziuba M. Life style and the risk of development of circulatory system diseases.

Borzecki A, Zolkowska D, Sieklucka-Dziuba M. Life style and the risk of development of circulatory system diseases. Study Design
Ann Univ Mariae Curie Sklodowska Med. 2002. 57:426-32 https://www.ncbi.nlm.nih.gov/pubmed/12898955

135 Boston RC, Moate PJ, Allison KC, Lundgren JD, Stunkard AJ. Modeling circadian rhythms of food intake by means of parametric deconvolution: results from studies of the night eating syndrome. Am J Clin Nutr. 2008. 87:1672-7 https://www.ncbi.nlm.nih.gov/pubmed/18541555 concentrations, and body composition in trained young men. Int J Sport Nutr Exerc Metab. 2008. 18:617-27 https://www.ncbi.nlm.nih.gov/pubmed/19164831

Study Design,

Intervention/Exposure, Daily Eating Occasions Not Reported

Intervention/Exposure, Daily Eating Occasions Not Reported 
137 Bouhlel E, Salhi Z, Bouhlel H, Mdella S, Amamou A, Zaouali M, Mercier J, Bigard X, Tabka Z, Zbidi A, Shephard RJ. Effect of Ramadan fasting on fuel oxidation during exercise in trained male rugby players. Diabetes Metab. 2006. 32:617-24 https://www.ncbi.nlm.nih.gov/pubmed/17296516

138 Bouhlel E, Zaouali M, Miled A, Tabka Z, Bigard X, Shephard R. Ramadan fasting and the GH/IGF-1 axis of trained men during submaximal exercise. Ann Nutr Metab. 2008. 52:261-6 https://www.ncbi.nlm.nih.gov/pubmed/18617733

139 Bouhlel H, Shephard RJ, Gmada N, Aouichaoui C, Peres G, Tabka Z, Bouhlel E. Effect of Ramadan observance on maximal muscular performance of trained men. Clin J Sport Med. 2013. 23:222-7 https://www.ncbi.nlm.nih.gov/pubmed/23160275

140 Bouida W, Beltaief K, Baccouche H, Sassi M, Dridi Z, Trabelsi I, Laaouiti K, Chakroun T, Hellara I, Boukef R, Sakly N, Hassine M, Added F, Razgallah R, Najjar F, Nouira S. Effects of Ramadan fasting on aspirin resistance in type 2 diabetic patients. PLoS One. 2018. 13:e0192590 https://www.ncbi.nlm.nih.gov/pubmed/29529091

141 Bowen J, Brindal E, James-Martin G, Noakes M. Randomized Trial of a High Protein, Partial Meal Replacement Program with or without Alternate Day Fasting: Similar Effects on Weight Loss, Retention Status, Nutritional, Metabolic, and Behavioral Outcomes. Nutrients. 2018;10(9). https://www.ncbi.nlm.nih.gov/pubmed/30142886.

142 Breakfast: pro and con. It's always been billed as the most important meal of the day-until now. Duke Med Health News. 2014. 20:6-7 https://www.ncbi.nlm.nih.gov/pubmed/25481929

143 Briatore L, Andraghetti G, Cordera R. Effect of two fasting periods of different duration on ghrelin response to a mixed meal. Nutr Metab Cardiovasc Dis. 2006. 16:471-6 https://www.ncbi.nlm.nih.gov/pubmed/17015184

144 Brikou D, Zannidi D, Karfopoulou E, Anastasiou CA, Yannakoulia M. Breakfast consumption and weight-loss maintenance: results from the MedWeight study. Br J Nutr. 2016. 115:2246-51 https://www.ncbi.nlm.nih.gov/pubmed/27185413

145 Brisswalter J, Bouhlel E, Falola JM, Abbiss CR, Vallier JM, Hausswirth C. Effects of Ramadan intermittent fasting on middle-distance running performance in well-trained runners. Clin J Sport Med. 2011. 21:422-7 https://www.ncbi.nlm.nih.gov/pubmed/21857506

146 Brophy S, Cooksey R, Gravenor MB, Mistry R, Thomas N, Lyons RA, Williams R. Risk factors for childhood obesity at age 5: analysis of the millennium cohort study. BMC Public Health. 2009. 9:467 https://www.ncbi.nlm.nih.gov/pubmed/20015353

Intervention/Exposure, Daily Eating Occasions Not Reported

Intervention/Exposure, Daily Eating Occasions Not Reported

Intervention/Exposure, Daily Eating Occasions Not Reported, Outcome

Health Status

Daily Eating Occasions Not

Reported, Comparator

Publication Status

Daily Eating Occasions Not Reported, Outcome

Daily Eating Occasions Not Reported

Daily Eating Occasions Not Reported, Outcome

Intervention/Exposure, Daily Eating Occasions Not Reported

Intervention/Exposure Bucher Della Torre S, Wild P, Dorribo V, Amati F, Danuser B. Eating Habits of Professional Firefighters: Comparison With National Guidelines and Impact Healthy Eating Promotion Program. J Occup Environ Med. 2019. 61:e183e190 https://www.ncbi.nlm.nih.gov/pubmed/31268938 


\section{Citation}

148

Burdge GC, Jones AE, Frye SM, Goodson L, Wootton SA. Effect of meal sequence on postprandial lipid, glucose and insulin responses in young men. Eur J Clin Nutr. 2003. 57:1536-44 https://www.ncbi.nlm.nih.gov/pubmed/14647218

149 Buyken AE, Trauner K, Günther ALB, Kroke A, Remer T. Breakfast glycemic index affects subsequent daily energy intake in free-living healthy children. American journal of clinical nutrition. 2007;86(4):980-7. https://www.ncbi.nlm.nih.gov/pubmed/17921374.

150 Buyukkurt A, Bourguignon C, Antinora C, Farquhar E, Gao X, Passarella E, Sibthorpe D, Gou K, Saury S, Beaulieu S, Storch KF, Linnaranta O. Irregular eating patterns associate with hypomanic symptoms in bipolar disorders. Nutr Neurosci. 2019. \#volume\#:1-12 https://www.ncbi.nlm.nih.gov/pubmed/30873919

151 Byrne N, Hills A, Salis A, King N, Wood R. Changes in total and activity energy expenditure accompanying continuous versus intermittent energy restriction: the matador study. Obesity facts. 2018. 11:93

152 Byrne N, Sainsbury A, Wood R, King N, Hills A. Intermittent energy restriction improves weight loss efficiency in obese men. Obesity reviews. 2014. 15:52

153 Byrne NM, Sainsbury A, King NA, Hills AP, Wood RE. Intermittent energy restriction improves weight loss efficiency in obese men: the MATADOR study. Int J Obes (Lond). 2018. 42:129-138 https://www.ncbi.nlm.nih.gov/pubmed/28925405

154 Cachelin FM, Thomas C, Vela A, Gil-Rivas V. Associations between meal patterns, binge eating, and weight for Latinas. Int J Eat Disord. 2017. 50:32-39 https://www.ncbi.nlm.nih.gov/pubmed/27436488

155 Calamaro CJ, Park S, Mason TB, Marcus CL, Weaver TE, Pack A, Ratcliffe SJ. Shortened sleep duration does not predict obesity in adolescents. J Sleep Res. 2010. 19:559-66 https://www.ncbi.nlm.nih.gov/pubmed/20545836

156 Cameron JD, Cyr MJ, Doucet E. Increased meal frequency does not promote greater weight loss in subjects who were prescribed an 8-week equi-energetic energy-restricted diet. Br J Nutr. 2010. 103:1098-101 https://www.ncbi.nlm.nih.gov/pubmed/19943985

157

Cansel M, Tasolar H, Yagmur J, Ermis N, Acikgoz N, Eyyupkoca F, Pekdemir H, Ozdemir R. The effects of Ramadan fasting on heart rate variability in healthy individuals: a prospective study. Anadolu Kardiyol Derg. 2014. 14:413-6 https://www.ncbi.nlm.nih.gov/pubmed/24901017

\section{Rationale}

Daily Eating Occasions Not

Reported, Size of Study Groups

Intervention/Exposure, Daily Eating Occasions Not Reported

Outcome, Health Status

Publication Status

Publication Status

Daily Eating Occasions Not

Reported

Study Design

Daily Eating Occasions Not Reported

Size of Study Groups

Daily Eating Occasions Not Reported

Intervention/Exposure, Daily Eating Occasions Not Reported, Outcome 

$\mathrm{C}$, Priore SE. Anthropometric and biochemical parameters in adolescents and their relationship with eating habits and household food availability. Nutr Hosp. 2013. 28:1352-6 https://www.ncbi.nlm.nih.gov/pubmed/23889666

160 Carels RA, Young KM, Coit C, Clayton AM, Spencer A, Wagner M. Skipping meals and alcohol consumption. The regulation of energy intake and expenditure among weight loss participants. Appetite. 2008. 51:538-45 https://www.ncbi.nlm.nih.gov/pubmed/18511146

161 Carey J, Neher J, St Anna L. Clinical Inquiry. Do dietary choices alone alter the risk of developing metabolic syndrome?. J Fam Pract. 2013. 62:507-19 https://www.ncbi.nlm.nih.gov/pubmed/24080561

162 Carlson JA, Crespo NC, Sallis JF, Patterson RE, Elder JP. Dietary-related and physical activity-related predictors of obesity in children: a 2-year prospective study. Child Obes. 2012. 8:110-5 https://www.ncbi.nlm.nih.gov/pubmed/22799510

163 Carlson O, Martin B, Stote KS, Golden E, Maudsley S, Najjar SS, Ferrucci L, Ingram DK, Longo DL, Rumpler WV, Baer DJ, Egan J, Mattson MP. Impact of reduced meal frequency without caloric restriction on glucose regulation in healthy, normal-weight middle-aged men and women. Metabolism. 2007. 56:1729-34 https://www.ncbi.nlm.nih.gov/pubmed/17998028

Carrard I, Rothen S. Factors associated with disordered eating behaviors and attitudes in older women. Eat Weight
Disord. 2019;1-9. https://www.ncbi.nlm.nih.gov/pubmed/30734223

165 Cassimos D, Sidiropoulos H, Batzios S, Balodima V, Christoforidis A. Sociodemographic and dietary risk factors for excess weight in a greek pediatric population living in Kavala, Northern Greece. Nutr Clin Pract. 2011. 26:186-91 https://www.ncbi.nlm.nih.gov/pubmed/21447773

166 Catenacci VA, Pan Z, Ostendorf D, Brannon S, Gozansky WS, Mattson MP, Martin B, MacLean PS, Melanson EL, Troy Donahoo W. A randomized pilot study comparing zero-calorie alternate-day fasting to daily caloric restriction in adults with obesity. Obesity (Silver Spring). 2016. 24:1874-83 https://www.ncbi.nlm.nih.gov/pubmed/27569118

167 Cayres SU, Urban JB, Fernandes RA. Physical Activity and Skipping Breakfast Have Independent Effects on Body Fatness Among Adolescents. J Pediatr Gastroenterol Nutr. 2018. 67:666-670 https://www.ncbi.nlm.nih.gov/pubmed/29952827

168 Ccps Janovsky, Laurinavicius A, Cesena F, Valente V, Ferreira CE, Mangueira C, Conceicao R, Santos RD, Bittencourt MS. Impact of self-reported fasting duration on lipid profile variability, cardiovascular risk stratification and metabolic syndrome diagnosis. Arch Endocrinol Metab. 2018. 62:187-192 https://www.ncbi.nlm.nih.gov/pubmed/29641736

Daily Eating Occasions Not Reported

\section{Publication Status}

Intervention/Exposure, Daily Eating Occasions Not Reported

\section{Eating Frequency Data}

Collection

Study Design,

Intervention/Exposure

\section{Study Design}

Daily Eating Occasions Not

Reported, Comparator

Daily Eating Occasions Not

Reported

Daily Eating Occasions Not Reported 

and diet quality: Contributors to childhood obesity. Obesity (Silver Spring). 2016. 24:184-90 https://www.ncbi.nlm.nih.gov/pubmed/26592489

171 Cha E, Talman MS, Massey AH, Yan F, Rogers AE. Sleep, Lifestyle Behaviors, and Cardiometabolic Health Markers in Overweight/Obese Young Adults: A Pilot Study Using the SenseWear® Armband. Biol Res Nurs. 2018. 20:541-548 https://www.ncbi.nlm.nih.gov/pubmed/30071743

172 Chan JL, Bullen J, Lee JH, Yiannakouris N, Mantzoros CS. Ghrelin levels are not regulated by recombinant leptin administration and/or three days of fasting in healthy subjects. J Clin Endocrinol Metab. 2004. 89:335-43 https://www.ncbi.nlm.nih.gov/pubmed/14715869

173 Chaouachi A, Chamari K, Roky R, Wong P, Mbazaa A, Bartagi Z, Amri M. Lipid profiles of judo athletes during Ramadan. Int J Sports Med. 2008. 29:282-8 https://www.ncbi.nlm.nih.gov/pubmed/17879887

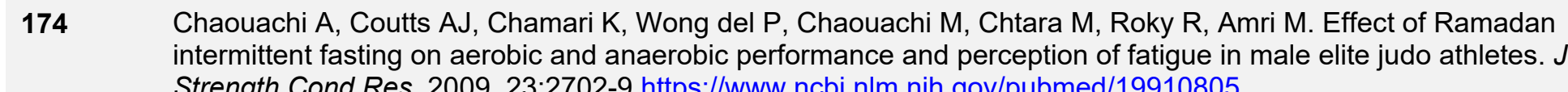
Strength Cond Res. 2009. 23:2702-9 https://www.ncbi.n/m.nih.gov/pubmed/19910805

Chapelot D, Marmonier C, Aubert R, Allegre C, Gausseres N, Fantino M, Louis-Sylvestre J. Consequence of omitting or adding a meal in man on body composition, food intake, and metabolism. Obesity (Silver Spring). 2006.

$175 \begin{aligned} & \text { Chapelot D, Marmonier C, Aubert R, Allegre C, Gausseres } \\ & \text { omitting or adding a meal in man on body composition, food } \\ & \text { 14:215-27 https://www.ncbi.nlm.nih.gov/pubmed/16571846 }\end{aligned}$

176 Chapelot D, Chapelot D. The role of snacking in energy balance: a biobehavioral approach. Journal of nutrition. 2011;141(1):158-62. https://www.ncbi.nlm.nih.gov/pubmed/21123465.

177 Chapuis-de-Andrade S, de Araujo RM, Lara DR. Association of weight control behaviors with body mass index and weight-based self-evaluation. Braz J Psychiatry. 2017. 39:237-243 https://www.ncbi.nlm.nih.gov/pubmed/28355342 https://www.ncbi.nlm.nih.gov/pubmed/21966060 
180 Chen CY, Hsiao YC. Dual trajectories of breakfast eating and fruit and vegetable intake over a 5-year follow-up period among economically disadvantaged children: Gender differences. Appetite. 2018. 121:41-49 https://www.ncbi.nlm.nih.gov/pubmed/29079477

181 Chen HJ, Wang Y, Cheskin LJ. Relationship between frequency of eating and cardiovascular disease mortality in U.S. adults: the NHANES III follow-up study. Ann Epidemiol. 2016. 26:527-533 https://www.ncbi.nlm.nih.gov/pubmed/27397905

182 Chennaoui M, Desgorces F, Drogou C, Boudjemaa B, Tomaszewski A, Depiesse F, Burnat P, Chalabi H, GomezMerino D. Effects of Ramadan fasting on physical performance and metabolic, hormonal, and inflammatory parameters in middle-distance runners. Appl Physiol Nutr Metab. 2009. 34:587-94 https://www.ncbi.nlm.nih.gov/pubmed/19767792

183 Cherif A, Meeusen R, Faroog A, Ryu J, Fenneni MA, Nikolovski Z, Elshafie S, Chamari K, Roelands B. Three Days of Intermittent Fasting: Repeated-Sprint Performance Decreased by Vertical-Stiffness Impairment. Int $J$ Sports Physiol Perform. 2017. 12:287-294 https://www.ncbi.nlm.nih.gov/pubmed/27248138

184 Chihaoui M, Grira W, Bettaieb J, Yazidi M, Chaker F, Rejeb O, Oueslati I, Feki M, Kaabachi N, Slimane H. The risk for hypoglycemia during Ramadan fasting in patients with adrenal insufficiency. Nutrition. 2018. 45:99-103 https://www.ncbi.nlm.nih.gov/pubmed/29129244

185 Cho AR, Moon JY, Kim S, An KY, Oh M, Jeon JY, Jung DH, Choi MH, Lee JW. Effects of alternate day fasting and exercise on cholesterol metabolism in overweight or obese adults: A pilot randomized controlled trial. Metabolism. 2019. 93:52-60 https://www.ncbi.nlm.nih.gov/pubmed/30615947

186 Chowdhury EA, Richardson JD, Holman GD, Tsintzas K, Thompson D, Betts JA. The causal role of breakfast in energy balance and health: a randomized controlled trial in obese adults. Am J Clin Nutr. 2016. 103:747-56 https://www.ncbi.nlm.nih.gov/pubmed/26864365

187 Chowdhury EA, Richardson JD, Tsintzas K, Thompson D, Betts JA. Carbohydrate-rich breakfast attenuates glycaemic, insulinaemic and ghrelin response to ad libitum lunch relative to morning fasting in lean adults. $\mathrm{Br} J$ Nutr. 2015. 114:98-107 https://www.ncbi.nlm.nih.gov/pubmed/26004166 libitum lunch intake and associated metabolic and hormonal responses in obese adults. Int $J$ Obes (Lond). 2016. 40:305-11 https://www.ncbi.nlm.nih.gov/pubmed/26278005

189 Chowdhury EA, Richardson JD, Tsintzas K, Thompson D, Betts JA. Postprandial Metabolism and Appetite Do Not Differ between Lean Adults that Eat Breakfast or Morning Fast for 6 Weeks. J Nutr. 2018. 148:13-21 https://www.ncbi.nlm.nih.gov/pubmed/29378040
Daily Eating Occasions Not

Reported, Duplicate

Eating Frequency Data Collection

Daily Eating Occasions Not Reported

Daily Eating Occasions Not Reported

Daily Eating Occasions Not Reported, Health Status

Daily Eating Occasions Not Reported

Daily Eating Occasions Not Reported

Daily Eating Occasions Not Reported

Daily Eating Occasions Not Reported

Daily Eating Occasions Not Reported, Size of Study Groups 

Excessive Postpartum Weight Retention: A Qualitative Interview Study. PLoS One. 2016. 11:e0167731 https://www.ncbi.nlm.nih.gov/pubmed/27936110

191 Ciangherotti S, Kevicka K, Ciangherotti E, Rondinone R. Eating behaviour: research in Liguria on young, adult and elderly subjects. J Prev Med Hyg. 2006. 47:26-36 https://www.ncbi.nlm.nih.gov/pubmed/17061408

192 Ciccone J, Woodruff SJ, Fryer K, Campbell T, Cole M. Associations among evening snacking, screen time, weight Study Design status, and overall diet quality in young adolescents. Appl Physiol Nutr Metab. 2013. 38:789-94 https://www.ncbi.nlm.nih.gov/pubmed/23980738

193 Clayton DJ, Biddle J, Maher T, Funnell MP, Sargeant JA, King JA, Hulston CJ, Stensel DJ, James LJ. 24-h severe energy restriction impairs postprandial glycaemic control in young, lean males. Br J Nutr. 2018. 120:1107-1116 https://www.ncbi.nlm.nih.gov/pubmed/30401004

194 Clayton DJ, Creese M, Skidmore N, Stensel DJ, James LJ. No effect of 24 h severe energy restriction on appetite regulation and ad libitum energy intake in overweight and obese males. Int J Obes (Lond). 2016. 40:1662-1670 https://www.ncbi.nlm.nih.gov/pubmed/27339607

195 Clayton DJ, Stensel DJ, James LJ. Effect of breakfast omission on subjective appetite, metabolism, acylated ghrelin and GLP-17-36 during rest and exercise. Nutrition. 2016. 32:179-85 https://www.ncbi.nlm.nih.gov/pubmed/26421384

Comparator, Size of Study Groups

\section{Daily Eating Occasions Not} Reported

Eating Frequency Data Collection, Size of Study Groups

196 Cleator J, Judd P, James M, Abbott J, Sutton CJ, Wilding JP. Characteristics and perspectives of night-eating behaviour in a severely obese population. Clin Obes. 2014. 4:30-8 https://www.ncbi.nlm.nih.gov/pubmed/25425130

197 Clifford LM, Beebe DW, Simon SL, Kuhl ES, Filigno SS, Rausch JR, Stark LJ. The association between sleep duration and weight in treatment-seeking preschoolers with obesity. Sleep Med. 2012. 13:1102-5 https://www.ncbi.nlm.nih.gov/pubmed/22841032

198 Colles SL, Dixon JB, O'Brien PE. Night eating syndrome and nocturnal snacking: association with obesity, binge eating and psychological distress. Int J Obes (Lond). 2007. 31:1722-30 https://www.ncbi.nlm.nih.gov/pubmed/17579633

199 Conley M, Le Fevre L, Haywood C, Proietto J. Is two days of intermittent energy restriction per week a feasible weight loss approach in obese males? A randomised pilot study. Nutr Diet. 2018. 75:65-72 https://www.ncbi.nlm.nih.gov/pubmed/28791787

Conti K. Nutrition status of American Indian adults and impending needs in view of the strong heart dietary study. $J$ Am Diet Assoc. 2008. 108:781-4 https://www.ncbi.nlm.nih.gov/pubmed/18442499

\section{Study Design}

Intervention/Exposure, Daily Eating Occasions Not Reported

Intervention/Exposure, Daily

Eating Occasions Not Reported

Daily Eating Occasions Not Reported

Study Design 
201

Coppinger T, Jeanes YM, Hardwick J, Reeves S. Body mass, frequency of eating and breakfast consumption in 9-

13-year-olds. J Hum Nutr Diet. 2012. 25:43-9 https://www.ncbi.nlm.nih.gov/pubmed/21649747

202

Corbalan-Tutau MD, Madrid JA, Garaulet M. Timing and duration of sleep and meals in obese and normal weight women. Association with increase blood pressure. Appetite. 2012. 59:9-16 https://www.ncbi.nlm.nih.gov/pubmed/22450522

203

Corder K, van Sluijs EM, Ridgway CL, Steele RM, Prynne CJ, Stephen AM, Bamber DJ, Dunn VJ, Goodyer IM,

Ekelund U. Breakfast consumption and physical activity in adolescents: daily associations and hourly patterns. Am J Clin Nutr. 2014. 99:361-8 https://www.ncbi.nlm.nih.gov/pubmed/24284440

204 Corder K, van Sluijs EM, Steele RM, Stephen AM, Dunn V, Bamber D, Goodyer I, Griffin SJ, Ekelund U. Breakfast consumption and physical activity in British adolescents. Br J Nutr. 2011. 105:316-21 https://www.ncbi.nlm.nih.gov/pubmed/20807464

Corley B, Khouri C, Theaude L, Hawke P, Hall R, Weatherall M, Krebs J. Changes in resting energy expenditure with intermittent fasting versus continuous daily restriction-a randomised controlled trial. Internal medicine journal. 2019. 49:5

206 Coronary artery disease. J Pract Nurs. 2008. 58:14-20 https://www.ncbi.nlm.nih.gov/pubmed/19260363

207 Correa-Arruda WS, Vaez IDA, Aguilar-Nascimento JE, Dock-Nascimento DB. Effects of overnight fasting on handgrip strength in inpatients. Einstein (Sao Paulo). 2019. 17:eAO4418 https://www.ncbi.nlm.nih.gov/pubmed/30652738

208 Coutinho SR, Glsbakk S, Halset EH, Kulseng B, Truby H, Martins C. Effect of intermittent versus continuous energy restriction on compensatory mechanisms activated during weight reduction. Obesity facts. 2015. 8:107

209 Coutinho SR, Halset EH, Gasbakk S, Rehfeld JF, Kulseng B, Truby H, Martins C. Compensatory mechanisms activated with intermittent energy restriction: A randomized control trial. Clin Nutr. 2018. 37:815-823 https://www.ncbi.nlm.nih.gov/pubmed/28446382

210 Cowley G. The ultimate diet plan. Don't eat so much. Newsweek. 2001. 137:53

211 Crimarco A, Turner-McGrievy GM, Wirth MD. The effects of meal-timing on self-rated hunger and dietary inflammatory potential among a sample of college students. J Am Coll Health. 2019. 67:328-337 https://www.ncbi.nlm.nih.gov/pubmed/29979952

Culnan E, Kloss JD, Grandner M. A prospective study of weight gain associated with chronotype among college freshmen. Chronobiol Int. 2013. 30:682-90 https://www.ncbi.nlm.nih.gov/pubmed/23688114
Study Design, Publication Status

Study Design

Study Design

Study Design

Study Design

Study Design, Publication Status

Study Design

Daily Eating Occasions Not

Reported

Publication Status

Daily Eating Occasions Not Reported, Size of Study Groups

Intervention/Exposure

Intervention/Exposure, Daily Eating Occasions Not Reported 


\section{3}

214 Daniels SR. Clues to concerns about eating and weight in adolescents. Journal of Pediatrics. 2010. 157:A2

Dannecker EA, Liu Y, Rector RS, Thomas TR, Sayers SP, Leeuwenburgh C, Ray BK. The effect of fasting on indicators of muscle damage. Exp Gerontol. 2013. 48:1101-6 https://www.ncbi.nlm.nih.gov/pubmed/23266375

215 D'Arrigo T. Snack attack. Night-eating syndrome is no joke. Diabetes Forecast. 2007. 60:20 https://www.ncbi.nlm.nih.gov/pubmed/17941142

216 Das SK, Saltzman E, Gilhooly CH, DeLany JP, Golden JK, Pittas AG, Dallal GE, Bhapkar MV, Fuss PJ, Dutta C, McCrory MA, Roberts SB. Low or moderate dietary energy restriction for long-term weight loss: what works best?. Obesity (Silver Spring). 2009. 17:2019-24 https://www.ncbi.nlm.nih.gov/pubmed/19390525

217 Dashti HS, Merino J, Lane JM, Song Y, Smith CE, Tanaka T, McKeown NM, Tucker C, Sun D, Bartz TM, Li-Gao R, Nisa H, Reutrakul S, Lemaitre RN, Alshehri TM, de Mutsert R, Bazzano L, Qi L, Knutson KL, Psaty BM, Mook-

Kanamori DO, Perica VB, Neuhouser ML, Fajl Scheer, Rutter MK, Garaulet M, Saxena R. Genome-wide association study of breakfast skipping links clock regulation with food timing. Am J Clin Nutr. 2019. 110:473-484 https://www.ncbi.nlm.nih.gov/pubmed/31190057

\section{8} Dattilo M, Crispim CA, Zimberg IZ, Tufik S, De Mello MT. Meal distribution across the day and its relationship with
body composition. Biological Rhythm Research. 2011. 42:119-129

\section{9} Davis AM, Canter KS, Stough CO, Gillette MD, Patton S. Measurement of mealtime behaviors in rural overweight children: an exploratory factor analysis of the Behavioral Pediatrics Feeding Assessment Scale. J Pediatr Psychol. 2014. 39:332-9 https://www.ncbi.nlm.nih.gov/pubmed/24326908 de Castro JM. 23:374-377 De Sousa A. Maternal, child and Metabolism. 2009. 17:111-112

222

Della Libera B, Ribeiro Baiao M, de Souza Santos MM, Padilha P, Dutra Alves P, Saunders C. Adherence of pregnant women to dietary counseling and adequacy of total gestational weight gain. Nutr Hosp. 2011. 26:79-85 https://www.ncbi.nlm.nih.gov/pubmed/21519732 https://www.ncbi.nlm.nih.gov/pubmed/30379452.
Deniz CD, Ozler S, Sayin FK, Eryilmaz MA. Associations between night eating syndrome and metabolic parameters in pregnant women. Turk J Obstet Gynecol. 2019. 16:107-111 https://www.ncbi.nlm.nih.gov/pubmed/31360584
Study Design

Daily Eating Occasions Not Reported, Comparator

Study Design, Publication Status

Intervention/Exposure, Daily Eating Occasions Not Reported

Intervention/Exposure, Daily Eating Occasions Not Reported, Outcome

\section{Study Design}

Study Design,

Intervention/Exposure, Daily Eating Occasions Not Reported

Study Design

Study Design

\section{Study Design}

Intervention/Exposure, Publication Status, Language Intervention/Exposure 

loss during a hypocaloric diet intervention in middle-aged and older adults. Obesity (Silver Spring). 2010. 18:300-7 https://www.ncbi.nlm.nih.gov/pubmed/19661958

226 Develioglu ON, Kucur M, Ipek HD, Celebi S, Can G, Kulekci M. Effects of Ramadan fasting on serum immunoglobulin $\mathrm{G}$ and $\mathrm{M}$, and salivary immunoglobulin A concentrations. J Int Med Res. 2013. 41:463-72 https://www.ncbi.nlm.nih.gov/pubmed/23569012 parameters among fasting and nonfasting workers during Ramadan in Indonesia. Eur J Clin Nutr. 2006. 60:877-81 https://www.ncbi.nlm.nih.gov/pubmed/16489329

228 Dhurandhar EJ, Dawson J, Alcorn A, Larsen LH, Thomas EA, Cardel M, Bourland AC, Astrup A, St-Onge MP, Hill JO, Apovian CM, Shikany JM, Allison DB. The effectiveness of breakfast recommendations on weight loss: a randomized controlled trial. Am J Clin Nutr. 2014. 100:507-13 https://www.ncbi.nlm.nih.gov/pubmed/24898236

229 Dikensoy E, Balat O, Cebesoy B, Ozkur A, Cicek H, Can G. Effect of fasting during Ramadan on fetal development and maternal health. J Obstet Gynaecol Res. 2008. 34:494-8 https://www.ncbi.nlm.nih.gov/pubmed/18937702

Intervention/Exposure, Daily Eating Occasions Not Reported

\section{Country}

Dikensoy E, Balat O, Cebesoy B, Ozkur A, Cicek H, Can G. The effect of Ramadan fasting on maternal serum lipids,

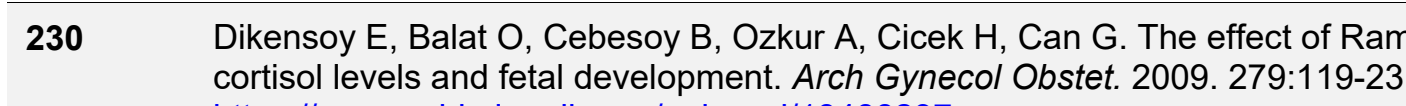
https://www.ncbi.nlm.nih.gov/pubmed/18488237

231 Dirks ML, Smeets JSJ, Holwerda AM, Kouw IWK, Marzuca-Nassr GN, Gijsen AP, Holloway GP, Verdijk LB, van Loon LJC. Dietary feeding pattern does not modulate the loss of muscle mass or the decline in metabolic health during short-term bed rest. Am J Physiol Endocrinol Metab. 2019. 316:E536-e545 https://www.ncbi.nlm.nih.gov/pubmed/30645176

\section{Dixit VD, Yang H, Sayeed KS, Stote KS, Rumpler WV, Baer DJ, Longo DL, Mattson MP, Taub DD. Controlled meal frequency without caloric restriction alters peripheral blood mononuclear cell cytokine production. $\mathrm{J}$ Inflamm (Lond). 2011. 8:6 https://www.ncbi.nlm.nih.gov/pubmed/21385360}

233 Dixon CB, Masteller B, Andreacci JL. The effect of a meal on measures of impedance and percent body fat estimated using contact-electrode bioelectrical impedance technology. Eur J Clin Nutr. 2013. 67:950-5 https://www.ncbi.nlm.nih.gov/pubmed/23820339

\section{Daily Eating Occasions Not} Reported

Intervention/Exposure, Daily Eating Occasions Not Reported

Intervention/Exposure, Daily Eating Occasions Not Reported

Intervention/Exposure,

Comparator

\section{Outcome}

Daily Eating Occasions Not Reported

Intervention/Exposure, Daily Eating Occasions Not Reported 


\section{Citation}

235

Donnelly LS, Shaw RL, Pegington M, Armitage CJ, Evans DG, Howell A, Harvie MN. 'For me it's about not feeling like I'm on a diet': a thematic analysis of women's experiences of an intermittent energy restricted diet to reduce breast cancer risk. J Hum Nutr Diet. 2018. 31:773-780 https://www.ncbi.nlm.nih.gov/pubmed/29926996

236 Dorflinger LM, Ruser CB, Masheb RM. Night eating among veterans with obesity. Appetite. 2017. 117:330-334 https://www.ncbi.nlm.nih.gov/pubmed/28711610

237

Dosamantes-Carrasco LD, Mendez-Hernandez P, Flores YN, Siani C, Denova-Gutierrez E, Gallegos-Carrillo K, Ramirez P, Rivera-Paredez B, Salazar-Martinez E, Salmeron J. Influence of mealtime habits on the risk of weight gain and obesity in Mexican adults. Public Health Nutr. 2017. 20:220-232 https://www.ncbi.nlm.nih.gov/pubmed/27667585

238

Doucet E, Imbeault P, St-Pierre S, Almeras N, Mauriege P, Richard D, Tremblay A. Appetite after weight loss by energy restriction and a low-fat diet-exercise follow-up. Int J Obes Relat Metab Disord. 2000. 24:906-14 https://www.ncbi.nlm.nih.gov/pubmed/10918539

239 Drapeau V, Jacob R, Panahi S, Tremblay A. Effect of Energy Restriction on Eating Behavior Traits and Psychobehavioral Factors in the Low Satiety Phenotype. Nutrients. 2019;11(2). https://www.ncbi.nlm.nih.gov/pubmed/30678317.

240 Drapeau V, Provencher V, Lemieux S, Despres JP, Bouchard C, Tremblay A. Do 6-y changes in eating behaviors predict changes in body weight? Results from the Quebec Family Study. Int J Obes Relat Metab Disord. 2003. 27:808-14 https://www.ncbi.nlm.nih.gov/pubmed/12821966

241 Dubois L, Girard M, Potvin Kent M. Breakfast eating and overweight in a pre-school population: is there a link?. Public Health Nutr. 2006. 9:436-42 https://www.ncbi.nlm.nih.gov/pubmed/16870015

242 Ducrot P, Mejean C, Bellisle F, Alles B, Hercberg S, Peneau S. Adherence to the French Eating Model is inversely associated with overweight and obesity: results from a large sample of French adults. Br J Nutr. 2018. 120:231-239 https://www.ncbi.nlm.nih.gov/pubmed/29781419

243 Duffin C. Tackling childhood obesity across London. Paediatr Nurs. 2009. 21:8-9 https://www.ncbi.nlm.nih.gov/pubmed/19623796

244

Duval K, Strychar I, Cyr MJ, Prud'homme D, Rabasa-Lhoret R, Doucet E. Physical activity is a confounding factor of the relation between eating frequency and body composition. Am J Clin Nutr. 2008. 88:1200-5 https://www.ncbi.nlm.nih.gov/pubmed/18996853

\section{Rationale}

Study Design, Size of Study Groups

Study Design,

Intervention/Exposure

Intervention/Exposure

Intervention/Exposure

Intervention/Exposure, Daily

Eating Occasions Not Reported

Intervention/Exposure

Daily Eating Occasions No

Reported

Study Design

Study Design,

Intervention/Exposure

Study Design

Eat your breakfast. When it comes to good nutrition, mom really does know best. Diabetes Forecast. 2006. 59:43-5 https://www.ncbi.nlm.nih.gov/pubmed/16779888 

https://www.ncbi.nlm.nih.gov/pubmed/16585927

247 Edinburgh RM, Hengist A, Smith HA, Travers RL, Betts JA, Thompson D, Walhin JP, Wallis GA, Hamilton DL, Stevenson EJ, Tipton KD, Gonzalez JT. Skipping Breakfast Before Exercise Creates a More Negative 24-hour Energy Balance: A Randomized Controlled Trial in Healthy Physically Active Young Men. J Nutr. 2019. 149:13261334 https://www.ncbi.nlm.nih.gov/pubmed/31321428

248 Effect of circadian restricted meal on blood pressure in young hypertensive individuals. Journal of hypertension. 2018. 36:e312

249 Effective dietary interventions for managing overweight and obesity in children. Nurs N Z. 2007. 13:30-1

https://www.ncbi.nlm.nih.gov/pubmed/17685044

250 Elands RJJ, Offermans NSM, Ccjm Simons, Schouten LJ, Verhage BA, van den Brandt PA, Weijenberg MP. Associations of adult-attained height and early life energy restriction with postmenopausal breast cancer risk according to estrogen and progesterone receptor status. Int J Cancer. 2019. 144:1844-1857 https://www.ncbi.nlm.nih.gov/pubmed/30252931 treating obesity. Jaapa. 2002. 15:11-2, 15-6, 19 passim https://www.ncbi.nlm.nih.gov/pubmed/12474429 adolescents. Soc Sci Med. 2016. 152:111-8 https://www.ncbi.nlm.nih.gov/pubmed/26851410

253 Elmehdawi R, Ehmida M, Elmagrehi H. Incidence of diabetic ketoacidosis during ramadan fasting in Benghazi-Libya. Health Status Oman Medical Journal. 2009;24(2):99-102. https://www.ncbi.nlm.nih.gov/pubmed/22334853.

254 El-Mitwalli A, Zaher AA, El Menshawi E. Circadian rhythm of stroke onset during the month of Ramadan. Acta Neurol Scand. 2010. 122:97-101 https://www.ncbi.nlm.nih.gov/pubmed/19839942

255 Enriquez E, Duncan GE, Schur EA. Age at dieting onset, body mass index, and dieting practices. A twin study. Appetite. 2013. 71:301-6 https://www.ncbi.nlm.nih.gov/pubmed/24025547

256 Erdem Y, Ozkan G, Ulusoy S, Arici M, Derici U, Sengul S, Sindel S, Erturk S. The effect of intermittent fasting on blood pressure variability in patients with newly diagnosed hypertension or prehypertension. J Am Soc Hypertens. 2018. 12:42-49 https://www.ncbi.nlm.nih.gov/pubmed/29275920
Intervention/Exposure, Daily Eating Occasions Not Reported

Daily Eating Occasions Not Reported

Intervention/Exposure, Daily Eating Occasions Not Reported

Study Design, Publication Status 

https://www.ncbi.nlm.nih.gov/pubmed/23497604

260 Eslamian L, Ramezani Z. Breakfast as a screening test for gestational diabetes. International Journal of Gynecology and Obstetrics. 2007;96(1):34-5. https://www.ncbi.nlm.nih.gov/pubmed/17188692. II cohort study. Diabet Med. 2019.https://www.ncbi.nlm.nih.gov/pubmed/30897241 design, and analysis plan of a behavioral weight management intervention for pregnant and postpartum women in the U.S. military. Contemporary clinical trials. 2018;74:46-54. https://www.ncbi.nlm.nih.gov/pubmed/30291998.

263 Fang CT, Chen VC, Ma HT, Chao HH, Ho MC, Gossop M. Attentional Bias, "Cool" and "Hot" Executive Functions in Obese Patients: Roles of Body Mass Index, Binge Eating, and Eating Style. J Clin Psychopharmacol. 2019. 39:145152 https://www.ncbi.nlm.nih.gov/pubmed/30742591

264 Faria-Schutzer DB, Surita FG, Rodrigues L, Turato ER. Eating Behaviors in Postpartum: A Qualitative Study of Women with Obesity. Nutrients. 2018;10(7). https://www.ncbi.nlm.nih.gov/pubmed/29996489.

265 Faris MA, Hussein RN, Al-Kurd RA, Al-Fararjeh MA, Bustanji YK, Mohammad MK. Impact of ramadan intermittent fasting on oxidative stress measured by urinary 15-f(2t)-isoprostane. J Nutr Metab. 2012. 2012:802924 https://www.ncbi.nlm.nih.gov/pubmed/23150812

266 Faris MAE, Madkour MI, Obaideen AK, Dalah EZ, Hasan HA, Radwan H, Jahrami HA, Hamdy O, Mohammad MG. Effect of Ramadan diurnal fasting on visceral adiposity and serum adipokines in overweight and obese individuals. Diabetes Res Clin Pract. 2019. 153:166-175 https://www.ncbi.nlm.nih.gov/pubmed/31150725

Daily Eating Occasions Not Reported

Daily Eating Occasions Not Reported

Intervention/Exposure, Daily Eating Occasions Not Reported 

https://www.ncbi.nlm.nih.gov/pubmed/15640455

270 Farshchi HR, Taylor MA, Macdonald IA. Regular meal frequency creates more appropriate insulin sensitivity and lipid profiles compared with irregular meal frequency in healthy lean women. Eur J Clin Nutr. 2004. 58:1071-7 https://www.ncbi.nlm.nih.gov/pubmed/15220950

271 Fawzi MH, Fawzi MM, Said NS, Fawzi MM, Fouad AA, Abdel-Moety H. Effect of Ramadan fasting on anthropometric, metabolic, inflammatory and psychopathology status of Egyptian male patients with schizophrenia. Psychiatry Res. 2015. 225:501-8 https://www.ncbi.nlm.nih.gov/pubmed/25529262 Discretionary Intakes in Australian Adults at Both Breakfast and the Rest of the Day. Nutrients. 2019;11(1). https://www.ncbi.nlm.nih.gov/pubmed/30650604

273 Feeley AB, Musenge E, Pettifor JM, Norris SA. Investigation into longitudinal dietary behaviours and household socio-economic indicators and their association with BMI Z-score and fat mass in South African adolescents: the Birth to Twenty (Bt20) cohort. Public Health Nutr. 2013. 16:693-703 https://www.ncbi.nlm.nih.gov/pubmed/22801035

274 Fehler KL, Kennedy LE, McCargar LJ, Bell RC, Ryan EA. Postpartum dietary changes in women with previous gestational diabetes mellitus. Canadian Journal of Diabetes. 2007. 31:54-61

275 Feig EH, Piers AD, Kral TVE, Lowe MR. Eating in the absence of hunger is related to loss-of-control eating, hedonic hunger, and short-term weight gain in normal-weight women. Appetite. 2018. 123:317-324 https://www.ncbi.nlm.nih.gov/pubmed/29331366

276 Feizollahzadeh S, Rasuli J, Kheirouri S, Alizadeh M. Augmented plasma adiponectin after prolonged fasting during ramadan in men. Health Promot Perspect. 2014. 4:77-81 https://www.ncbi.nlm.nih.gov/pubmed/25097840

\section{Comparator}

Size of Study Groups

Daily Eating Occasions Not

Reported, Health Status

\section{Study Design}

Daily Eating Occasions Not

Reported

Intervention/Exposure, Daily Eating Occasions Not Reported

Intervention/Exposure, Daily Eating Occasions Not Reported

Intervention/Exposure, Daily Eating Occasions Not Reported

277 Feller S, Muller A, Mayr A, Engeli S, Hilbert A, de Zwaan M. What distinguishes weight loss maintainers of the German Weight Control Registry from the general population?. Obesity (Silver Spring). 2015. 23:1112-8 https://www.ncbi.nlm.nih.gov/pubmed/25864871 
$279 \quad$ Fernandez Morales I, Aguilar Vilas MV, Mateos Vega CJ, Martinez Para MC. Breakfast quality and its relationship to Study Design the prevalence of overweight and obesity in adolescents in Guadalajara (Spain). Nutr Hosp. 2011. 26:952-8 https://www.ncbi.nlm.nih.gov/pubmed/22072337

280 Ferraris C, Marcolin G, Veneto A, Tagliabue A, Paoli A. Time restricted feeding in high-level athletes: a pilot study. Nutrition, metabolism and cardiovascular diseases. 2019. 29:877-878

281 Ferreira SR, Gimeno SG, Hirai AT, Harima H, Matsumura L, Pittito Bde A. Effects of an intervention in eating habits and physical activity in Japanese-Brazilian women with a high prevalence of metabolic syndrome in Bauru, Sao Paulo State, Brazil. Cad Saude Publica. 2008. 24 Suppl 2:S294-302 https://www.ncbi.nlm.nih.gov/pubmed/18670709

\section{2 \\ Ferrie JE, Kivimaki M, Akbaraly TN, Tabak A, Abell J, Davey Smith G, Virtanen M, Kumari M, Shipley MJ. Change in} Sleep Duration and Type 2 Diabetes: The Whitehall II Study. Diabetes Care. 2015. 38:1467-72 https://www.ncbi.nlm.nih.gov/pubmed/26068863

283 Field AE, Austin SB, Gillman MW, Rosner B, Rockett HR, Colditz GA. Snack food intake does not predict weight change among children and adolescents. Int J Obes Relat Metab Disord. 2004. 28:1210-6 https://www.ncbi.nlm.nih.gov/pubmed/15314623

284 Field AE, Austin SB, Taylor CB, Malspeis S, Rosner B, Rockett HR, Gillman MW, Colditz GA. Relation between dieting and weight change among preadolescents and adolescents. Pediatrics. 2003. 112:900-6 https://www.ncbi.nlm.nih.gov/pubmed/14523184

285 Fineberg HV. An economic analysis of eating and physical activity behaviors: exploring effective strategies to combat obesity. Am J Prev Med. 2004. 27:172-4 https://www.ncbi.nlm.nih.gov/pubmed/15450628

286 Finlayson G, Cecil J, Higgs S, Hill A, Hetherington M. Susceptibility to weight gain. Eating behaviour traits and physical activity as predictors of weight gain during the first year of university. Appetite. 2012. 58:1091-8 https://www.ncbi.nlm.nih.gov/pubmed/22407132

287 Firouzbakht M, Kiapour A, Jamali B, Kazeminavaei F, Taghlilin F, Ali Morad Heidari G. Fasting in pregnancy: A survey of beliefs and manners of Muslim women about Ramadan fasting. Annals of Tropical Medicine \& Public Health. 2013;6(5):536-40. 2010. 25:221-2 https://www.ncbi.nlm.nih.gov/pubmed/20386244

Intervention/Exposure, Daily Eating Occasions Not Reported

Intervention/Exposure, Daily Eating Occasions Not Reported

Study Design

Intervention/Exposure

Study Design, Daily Eating

Occasions Not Reported

Study Design

Size of Study Groups 
290 Ford AL, Bergh C, Sodersten P, Sabin MA, Hollinghurst S, Hunt LP, Shield JP. Treatment of childhood obesity by retraining eating behaviour: randomised controlled trial. Bmj. 2009. 340:b5388

Intervention/Exposure, Daily https://www.ncbi.nlm.nih.gov/pubmed/20051465

291 Forkert ECO, Moraes ACF, Carvalho HB, Manios Y, Widhalm K, Gonzalez-Gross M, Gutierrez A, Kafatos A, Censi L, De Henauw S, Moreno LA. Skipping breakfast is associated with adiposity markers especially when sleep time is adequate in adolescents. Sci Rep. 2019. 9:6380 https://www.ncbi.nlm.nih.gov/pubmed/31011180

292 Fournier T, Tibere L, Laporte C, Mognard E, Ismail MN, Sharif SP, Poulain JP. Eating patterns and prevalence of obesity. Lessons learned from the Malaysian Food Barometer. Appetite. 2016. 107:362-371 https://www.ncbi.nlm.nih.gov/pubmed/27521166

293 Francis LA, Lee Y, Birch LL. Parental weight status and girls' television viewing, snacking, and body mass indexes. Obes Res. 2003. 11:143-51 https://www.ncbi.nlm.nih.gov/pubmed/12529497

Eating Occasions Not Reported

Study Design

294 Franko DL, Striegel-Moore RH, Thompson D, Affenito SG, Schreiber GB, Daniels SR, Crawford PB. The relationship between meal frequency and body mass index in black and white adolescent girls: more is less. Int $J$ Obes (Lond). 2008. 32:23-9 https://www.ncbi.nlm.nih.gov/pubmed/17563764

295 Freitas Junior IF, Christofaro DG, Codogno JS, Monteiro PA, Silveira LS, Fernandes RA. The association between skipping breakfast and biochemical variables in sedentary obese children and adolescents. J Pediatr. 2012. 161:871-4 https://www.ncbi.nlm.nih.gov/pubmed/22682613 and amount of recent weight loss in a sample of successful weight losers. Int J Behav Nutr Phys Act. 2012. 9:79 https://www.ncbi.nlm.nih.gov/pubmed/22734914

297 Fujiwara T. Skipping breakfast is associated with dysmenorrhea in young women in Japan. Int J Food Sci Nutr. 2003. 54:505-9 https://www.ncbi.nlm.nih.gov/pubmed/14522696

Daily Eating Occasions Not

Reported

\section{Study Design}

Study Design

Study Design

Study Design,

Intervention/Exposure, Daily

Eating Occasions Not Reported,

Outcome

298 Fullick S, Morris C, Jones H, Atkinson G. Prior exercise lowers blood pressure during simulated night-work with different meal schedules. American journal of hypertension. 2009;22(8):835-41.

https://www.ncbi.nlm.nih.gov/pubmed/19556971. students in Slovakia as one of the cardiovascular disease risk factors. Homeostasis in Health and Disease. 2004. 43:106-108 
301 Gabel K, Hoddy KK, Haggerty N, Song J, Kroeger CM, Trepanowski JF, Panda S, Varady KA. Effects of 8-hour time restricted feeding on body weight and metabolic disease risk factors in obese adults: A pilot study. Nutr Healthy

Daily Eating Occasions Not Aging. 2018. 4:345-353 https://www.ncbi.nlm.nih.gov/pubmed/29951594 2019. 44:107-109 https://www.ncbi.nlm.nih.gov/pubmed/30216730

Daily Eating Occasions Not Reported

303 Gabel K, Kroeger CM, Trepanowski JF, Hoddy KK, Cienfuegos S, Kalam F, Varady KA. Differential Effects of Alternate-Day Fasting Versus Daily Calorie Restriction on Insulin Resistance. Obesity (Silver Spring). 2019. 27:1443-1450 https://www.ncbi.nlm.nih.gov/pubmed/31328895

304 Gail Gates, Perera T. Association between Breakfast Consumption and Nutritional Status in 9 to 13 Year Old Children. Journal of Nutrition Education \& Behavior. 2013. 45:S31-S31

Intervention/Exposure, Daily Eating Occasions Not Reported

Study Design, Publication Status

305 Gallant A, Drapeau V, Allison KC, Tremblay A, Lambert M, O'Loughlin J, Lundgren JD. Night eating behavior and metabolic heath in mothers and fathers enrolled in the QUALITY cohort study. Eat Behav. 2014. 15:186-91 https://www.ncbi.nlm.nih.gov/pubmed/24854802

Gallant A, Lundgren J, O'Loughlin J, Allison K, Tremblay A, Henderson M, Drapeau V. Night-eating symptoms and 2-year weight change in parents enrolled in the QUALITY cohort. Int J Obes (Lond). 2015. 39:1161-5 https://www.ncbi.nlm.nih.gov/pubmed/25797608

307 Galson SK. Childhood overweight and obesity prevention. Public Health Rep. 2008. 123:258-9 https://www.ncbi.nlm.nih.gov/pubmed/19006963

308 Garaulet M, Gomez-Abellan P, Alburquerque-Bejar JJ, Lee YC, Ordovas JM, Scheer FA. Timing of food intake predicts weight loss effectiveness. Int J Obes (Lond). 2013. 37:604-11 https://www.ncbi.nlm.nih.gov/pubmed/23357955 Kafatos A, Molnar D, Al-Tahan J, Moreno LA. Short sleep duration is associated with increased obesity markers in European adolescents: effect of physical activity and dietary habits. The HELENA study. Int J Obes (Lond). 2011. 35:1308-17 https://www.ncbi.nlm.nih.gov/pubmed/21792170 Mediterranean) study. Am J Clin Nutr. 2016. 104:1160-1166 https://www.ncbi.nlm.nih.gov/pubmed/27629052

\section{Study Design}

Intervention/Exposure, Daily Eating Occasions Not Reported

\section{Study Design}

Intervention/Exposure

Study Design

Intervention/Exposure, Daily Eating Occasions Not Reported 


\section{Citation}

311 Geliebter A, Astbury NM, Aviram-Friedman R, Yahav E, Hashim S. Skipping breakfast leads to weight loss but also elevated cholesterol compared with consuming daily breakfasts of oat porridge or frosted cornflakes in overweight individuals: a randomised controlled trial. J Nutr Sci. 2014. 3:e56 https://www.ncbi.nlm.nih.gov/pubmed/26101624

312 Geliebter A, Gluck ME, Tanowitz M, Aronoff NJ, Zammit GK. Work-shift period and weight change. Nutrition. 2000. 16:27-9 https://www.ncbi.nlm.nih.gov/pubmed/10674231

313 Gerage AM, Benedetti TRB, Ritti-Dias RM, Dos Santos ACO, de Souza BCC, Almeida FA. Effectiveness of a Behavior Change Program on Physical Activity and Eating Habits in Patients With Hypertension: A Randomized Controlled Trial. J Phys Act Health. 2017. 14:943-952 https://www.ncbi.nlm.nih.gov/pubmed/28682707

\section{4}

Gibson P. Childhood obesity: an approach to weight management. J Fam Health Care. 2002. 12:88-9 https://www.ncbi.nlm.nih.gov/pubmed/12416013

315 Gilardini L, Croci M, Pasqualinotto L, Caffetto K, Invitti C. Dietary Habits and Cardiometabolic Health in Obese Children. Obes Facts. 2015. 8:101-9 https://www.ncbi.nlm.nih.gov/pubmed/26087840

316 Gill S, Panda S. A Smartphone App Reveals Erratic Diurnal Eating Patterns in Humans that Can Be Modulated for Health Benefits. Cell Metab. 2015. 22:789-98 https://www.ncbi.nlm.nih.gov/pubmed/26411343

317 Gingras V, Rifas-Shiman SL, Taveras EM, Oken E, Hivert MF. Dietary behaviors throughout childhood are associated with adiposity and estimated insulin resistance in early adolescence: a longitudinal study. Int $J$ Behav Nutr Phys Act. 2018. 15:129 https://www.ncbi.nlm.nih.gov/pubmed/30558613

318 Giorgio C, Monica M, Margherita T, Elisabetta F, Filippo R. Study of the eating habits of the students attending the first 2 years at high school in Italy. Mediterranean Journal of Nutrition and Metabolism. 2013. 6:143-150

319 Giudice R, Izzo R, Manzi MV, Pagnano G, Santoro M, Rao MA, Di Renzo G, De Luca N, Trimarco V. Lifestylerelated risk factors, smoking status and cardiovascular disease. High Blood Press Cardiovasc Prev. 2012. 19:85-92 https://www.ncbi.nlm.nih.gov/pubmed/22867094

320 Gluck ME, Geliebter A, Satov T. Night eating syndrome is associated with depression, low self-esteem, reduced daytime hunger, and less weight loss in obese outpatients. Obes Res. 2001. 9:264-7 https://www.ncbi.nlm.nih.gov/pubmed/11331430

321 Gluck ME, Venti CA, Salbe AD, Krakoff J. Nighttime eating: Commonly observed and related to weight gain in an inpatient food intake study. American journal of clinical nutrition. 2008;88(4):900-5.

https://www.ncbi.nlm.nih.gov/pubmed/18842774.

\section{Rationale}

Daily Eating Occasions Not Reported

Intervention/Exposure, Daily Eating Occasions Not Reported

Intervention/Exposure

Study Design

Study Design

Study Design,

Intervention/Exposure, Outcome

Daily Eating Occasions Not Reported

Study Design, Daily Eating Occasions Not Reported

Study Design

Intervention/Exposure, Daily Eating Occasions Not Reported

Daily Eating Occasions Not Reported 

homeostasis and adiponectin levels in healthy adult males. J Diabetes Metab Disord. 2015. 14:55 https://www.ncbi.nlm.nih.gov/pubmed/26155596

\section{Godfrey JR, Dansinger ML. Toward optimal health: sorting out the dietary approaches to achieve a healthy weight. $J$ Study Design} Womens Health (Larchmt). 2009. 18:435-8 https://www.ncbi.nlm.nih.gov/pubmed/19361308

324 Godlin Jeneta J, Preetha. Effect of breakfast in body mass index among college going students. Journal of pharmaceutical sciences and research. 2016. 8:545-551

Intervention/Exposure, Daily Eating Occasions Not Reported

325 Goel N, Hopkins C, Ruggieri M, Ahima RS, Allison KC. Delayed eating adversely impacts weight and metabolism compared with daytime eating in normal weight adults. Sleep. 2017. 40:A24-A25

Study Design, Publication Status

Goel N, Hopkins C, Ruggieri M, Zhang Z, Taylor DM, Allison KC. The impact of nighttime eating: a randomized
controlled trial of daytime vs. delayed eating on weight and metabolism in adults of normal weight. Sleep. 2019.

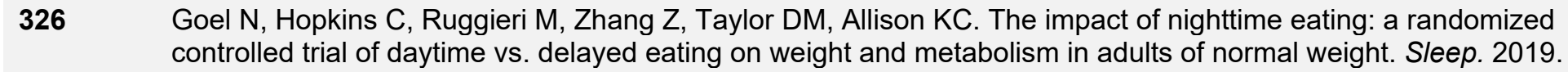
42:A15

327 Goel N, Stunkard AJ, Rogers NL, Van Dongen HP, Allison KC, O’Reardon JP, Ahima RS, Cummings DE, Heo M, Dinges DF. Circadian rhythm profiles in women with night eating syndrome. J Biol Rhythms. 2009. 24:85-94 https://www.ncbi.nlm.nih.gov/pubmed/19150931

328 Goff LM, Huang P, Silva MJ, Bordoli C, Enayat EZ, Molaodi OR, Cassidy A, Maynard M, Harding S. Associations of dietary intake with cardiometabolic risk in a multi-ethnic cohort: a longitudinal analysis of the Determinants of Adolescence, now young Adults, Social well-being and Health (DASH) study. Br J Nutr. 2019. 121:1069-1079 https://www.ncbi.nlm.nih.gov/pubmed/30764887

329 Goldhamer AC, Lisle DJ, Sultana P, Anderson SV, Parpia B, Hughes B, Campbell TC. Medically supervised wateronly fasting in the treatment of borderline hypertension. J Altern Complement Med. 2002. 8:643-50 https://www.ncbi.nlm.nih.gov/pubmed/12470446

330 Gonzalez JT, Richardson JD, Chowdhury EA, Koumanov F, Holman GD, Cooper S, Thompson D, Tsintzas K, Betts JA. Molecular adaptations of adipose tissue to 6 weeks of morning fasting vs. daily breakfast consumption in lean and obese adults. J Physiol. 2018. 596:609-622 https://www.ncbi.nlm.nih.gov/pubmed/29193093

Study Design, Publication Status

Gonzalez-Gonzalez A, Betancourt-Ocampo D, Tavel-Gelrud D, Martinez-Lanz P. Risk eating behaviors in male and
female students: a longitudinal study. Eat Behav. 2014. 15:252-4 https://www.ncbi.nlm.nih.gov/pubmed/24854813

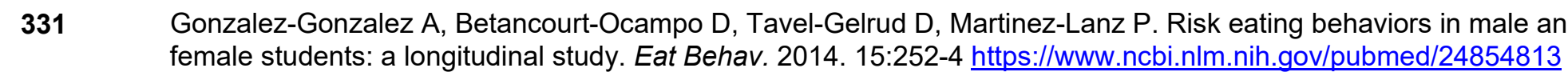

Daily Eating Occasions Not Reported

Daily Eating Occasions Not Reported, Eating Frequency Data Collection

Intervention/Exposure, Daily Eating Occasions Not Reported

Intervention/Exposure, Daily Eating Occasions Not Reported, Size of Study Groups

Intervention/Exposure

Study Design 
333 Gorin AA, Phelan S, Wing RR, Hill JO. Promoting long-term weight control: does dieting consistency matter?. Int J Obes Relat Metab Disord. 2004. 28:278-81 https://www.ncbi.nlm.nih.gov/pubmed/14647183

334 Goto M, Kiyohara K, Kawamura T. Lifestyle risk factors for overweight in Japanese male college students. Public Health Nutr. 2010. 13:1575-80 https://www.ncbi.nlm.nih.gov/pubmed/20025829

335 Goyal R, Julka S. Impact of breakfast skipping on the health status of the population. Indian J Endocrinol Metab. 2014. 18:683-7 https://www.ncbi.nlm.nih.gov/pubmed/25285286

336 Grant C, Coates A, Dorrian J, Kennaway D, Wittert G, Heilbronn L, et al. Eating on simulated night shift effects glucose response to breakfast: pilot study. FASEB journal. 2016;30

337 Grant C, Coates A, Dorrian J, Kennaway D, Wittert G, Heilbronn L, Pajcin M, Della Vedova C, Gupta C, Banks S. Fasting during night shift: a strategy to reduce the metabolic impact of shift-work. Sleep. 2016. 39:A51

338 Grant CL, Coates AM, Dorrian J, Kennaway DJ, Wittert GA, Heilbronn LK, Pajcin M, Della Vedova C, Gupta CC, Banks S. Timing of food intake during simulated night shift impacts glucose metabolism: A controlled study. Chronobiol Int. 2017. 34:1003-1013 https://www.ncbi.nlm.nih.gov/pubmed/28635334

339 Gray-Donald K, Robinson E, Collier A, David K, Renaud L, Rodrigues S. Intervening to reduce weight gain in pregnancy and gestational diabetes mellitus in Cree communities: an evaluation. Cmaj. 2000. 163:1247-51 https://www.ncbi.nlm.nih.gov/pubmed/11107459

340 Grilo CM, Masheb RM. Night-time eating in men and women with binge eating disorder. Behav Res Ther. 2004. 42:397-407 https://www.ncbi.nlm.nih.gov/pubmed/14998734

341 Gueldich H, Zghal F, Borji R, Chtourou H, Sahli S, Rebai H. The effects of Ramadan intermittent fasting on the underlying mechanisms of force production capacity during maximal isometric voluntary contraction. Chronobiol Int. 2019. 36:698-708 https://www.ncbi.nlm.nih.gov/pubmed/30889992

342 Guelinckx I, Devlieger R, Mullie P, Vansant G. Effect of lifestyle intervention on dietary habits, physical activity, and gestational weight gain in obese pregnant women: a randomized controlled trial. Am J Clin Nutr. 2010. 91:373-80 https://www.ncbi.nlm.nih.gov/pubmed/19955397

343 Guinter MA, Park YM, Steck SE, Sandler DP. Day-to-day regularity in breakfast consumption is associated with weight status in a prospective cohort of women. Int J Obes (Lond). 2019;44(1):186-94. https://www.ncbi.nlm.nih.gov/pubmed/30926951.
Gupta CC, Centofanti S, Dorrian J, Coates AM, Stepien JM, Kennaway D, et al. Subjective Hunger, Gastric Upset, and Sleepiness in Response to Altered Meal Timing during Simulated Shiftwork. Nutrients. 2019;11(6). https://www.ncbi.nlm.nih.gov/pubmed/31208092.
Intervention/Exposure, Daily Eating Occasions Not Reported

Intervention/Exposure, Daily Eating Occasions Not Reported

Study Design, Daily Eating Occasions Not Reported

Study Design, Publication Status

Publication Status

Intervention/Exposure, Daily Eating Occasions Not Reported, Comparator

Intervention/Exposure

Health Status

Daily Eating Occasions Not

Reported

Intervention/Exposure

Daily Eating Occasions Not

Reported

Comparator, Outcome 
$345 \quad$ Gupta M, Khajuria V, Akhter P. Effects of ramadan fasting on cardiovascular and biochemical parameters. Indian Journal of Community Health. 2013. 25:460-464

346 Gur EB, Turan GA, Ince O, Karadeniz M, Tatar S, Kasap E, Sahin N, Guclu S. Effect of Ramadan fasting on metabolic markers, dietary intake and abdominal fat distribution in pregnancy. Hippokratia. 2015. 19:298-303 https://www.ncbi.nlm.nih.gov/pubmed/27688692

347 Gwin JA, Leidy HJ. A pilot study assessing whether the consumption of a protein-rich breakfast improves appetite control, eating behavior, and sleep quality compared to skipping breakfast in healthy young professionals. Sleep. 2017. 40:A300

348 Gwin JA, Leidy HJ. Breakfast Consumption Augments Appetite, Eating Behavior, and Exploratory Markers of Sleep Quality Compared with Skipping Breakfast in Healthy Young Adults. Curr Dev Nutr. 2018. 2:nzy074 https://www.ncbi.nlm.nih.gov/pubmed/30402594

\section{9} Ha SA, Lee SY, Kim KA, Seo JS, Sohn CM, Park HR, Kim KW. Eating habits, physical activity, nutrition knowledge, and self-efficacy by obesity status in upper-grade elementary school students. Nutr Res Pract. 2016. 10:597-605 https://www.ncbi.nlm.nih.gov/pubmed/27909557

$350 \quad$ Haakstad LA, Voldner N, Bo K. Attitudes and awareness towards weight gain among normal weight and overweight pregnant women. J Matern Fetal Neonatal Med. 2015. 28:1569-74 https://www.ncbi.nlm.nih.gov/pubmed/25184626

$351 \quad$ Haas K, Hayoz S, Maurer-Wiesner S. Effectiveness and Feasibility of a Remote Lifestyle Intervention by Dietitians for Overweight and Obese Adults: Pilot Study. JMIR Mhealth Uhealth. 2019. 7:e12289 https://www.ncbi.nlm.nih.gov/pubmed/30973338

352 Haerens L, Vereecken C, Maes L, De Bourdeaudhuij I. Relationship of physical activity and dietary habits with body mass index in the transition from childhood to adolescence: a 4-year longitudinal study. Public Health Nutr. 2010. 13:1722-8 https://www.ncbi.nlm.nih.gov/pubmed/20883572

$353 \quad$ Hager ER, Calamaro CJ, Bentley LM, Hurley KM, Wang Y, Black MM. Nighttime Sleep Duration and Sleep Behaviors among Toddlers from Low-Income Families: Associations with Obesogenic Behaviors and Obesity and the Role of Parenting. Child Obes. 2016. 12:392-400 https://www.ncbi.nlm.nih.gov/pubmed/27447782

$354 \quad$ Haghdoost AA, Poorranjbar M. The interaction between physical activity and fasting on the serum lipid profile during Ramadan. Singapore Med J. 2009. 50:897-901 https://www.ncbi.nlm.nih.gov/pubmed/19787180

$355 \quad$ Haines J, Stang J. Promoting meal consumption among teens. J Am Diet Assoc. 2005. 105:945-7 https://www.ncbi.nlm.nih.gov/pubmed/15942546
Daily Eating Occasions Not Reported

Intervention/Exposure, Daily

Eating Occasions Not Reported

Study Design, Publication Status

Outcome, Size of Study Groups

Study Design

Intervention/Exposure

Intervention/Exposure

Daily Eating Occasions Not

Reported

Study Design,

Intervention/Exposure

Intervention/Exposure, Daily Eating Occasions Not Reported

Study Design, Publication Status 


\section{Citation}

356

Hajek $\mathrm{P}$, Myers K, Dhanji AR, West $\mathrm{O}$, McRobbie $\mathrm{H}$. Weight change during and after Ramadan fasting. J Public Health (Oxf). 2012. 34:377-81 https://www.ncbi.nlm.nih.gov/pubmed/22083256

357 Halberg N, Henriksen M, Soderhamn N, Stallknecht B, Ploug T, Schjerling P, Dela F. Effect of intermittent fasting and refeeding on insulin action in healthy men. J Appl Physiol. 2005. 99:2128-36 https://www.ncbi.nlm.nih.gov/pubmed/16051710

$358 \quad$ Halsey LG, Huber JW, Low T, Ibeawuchi C, Woodruff P, Reeves S. Does consuming breakfast influence activity levels? An experiment into the effect of breakfast consumption on eating habits and energy expenditure. Public Health Nutr. 2012. 15:238-45 https://www.ncbi.nlm.nih.gov/pubmed/21729464

359 Haluzik M, Matoulek M, Svacina S, Hilgertova J, Haas T. The influence of short-term fasting on serum leptin levels, and selected hormonal and metabolic parameters in morbidly obese and lean females. Endocr Res. 2001. 27:25160 https://www.ncbi.nlm.nih.gov/pubmed/11428717

$360 \quad$ Hamed IM, Hussein MM, El-Damhougy ST. The effect of the Coptic Orthodox Christian fasting on dietary intake and some biochemical parameters. Research Journal of Pharmaceutical, Biological and Chemical Sciences. 2016. 7:539-543

$361 \quad H a m e r m e s h$ DS. Incentives, time use and BMI: The roles of eating, grazing and goods. Econ Hum Biol. 2010. 8:2-15 https://www.ncbi.nlm.nih.gov/pubmed/20079698

362 Hammouda O, Chtourou H, Aloui A, Chahed H, Kallel C, Miled A, Chamari K, Chaouachi A, Souissi N. Concomitant effects of Ramadan fasting and time-of-day on apolipoprotein Al, B, Lp-a and homocysteine responses during aerobic exercise in Tunisian soccer players. PLoS One. 2013. 8:e79873 https://www.ncbi.nlm.nih.gov/pubmed/24244572

Hampl JS, Heaton CL, Taylor CA. Snacking patterns influence energy and nutrient intakes but not body mass index. J Hum Nutr Diet. 2003. 16:3-11 https://www.ncbi.nlm.nih.gov/pubmed/12581404 caloric consumption, body weight, and circadian evolution of cortisol serum levels in young, healthy male volunteers. Horm Metab Res. 2008. 40:575-7 https://www.ncbi.nlm.nih.gov/pubmed/18393168

Harder-Lauridsen NM, Nielsen ST, Mann SP, Lyngbaek MP, Benatti FB, Langkilde AR, Law I, Wedell-Neergaard AS, Thomsen C, Moller K, Karstoft K, Pedersen BK, Krogh-Madsen R. The effect of alternate-day caloric restriction on the metabolic consequences of 8 days of bed rest in healthy lean men: a randomized trial. J Appl Physiol. 2017. 122:230-241 https://www.ncbi.nlm.nih.gov/pubmed/27881670

\section{Rationale}

Daily Eating Occasions Not Reported

Daily Eating Occasions Not Reported, Size of Study Groups

Intervention/Exposure, Daily Eating Occasions Not Reported

Intervention/Exposure, Daily Eating Occasions Not Reported

Intervention/Exposure, Daily Eating Occasions Not Reported, Publication Status

Study Design, Daily Eating Occasions Not Reported

Daily Eating Occasions Not Reported

Study Design

Daily Eating Occasions Not Reported

Daily Eating Occasions Not Reported, Publication Date 
$366 \quad$ Harder-Lauridsen NM, Rosenberg A, Benatti FB, Damm JA, Thomsen C, Mortensen EL, Pedersen BK, KroghMadsen R. Ramadan model of intermittent fasting for $28 \mathrm{~d}$ had no major effect on body composition, glucose metabolism, or cognitive functions in healthy lean men. Nutrition. 2017. 37:92-103 https://www.ncbi.nlm.nih.gov/pubmed/28359370

367 Hart CN, Carskadon MA, Demos KE, Van Reen E, Sharkey KM, Raynor HA, Considine RV, Jones RN, Wing RR. Acute Changes in Sleep Duration on Eating Behaviors and Appetite-Regulating Hormones in Overweight/Obese Adults. Behav Sleep Med. 2015. 13:424-36 https://www.ncbi.nlm.nih.gov/pubmed/25105727

368 Hartline-Grafton HL, Rose D, Johnson CC, Rice JC, Webber LS. The influence of weekday eating patterns on energy intake and BMI among female elementary school personnel. Obesity (Silver Spring). 2010. 18:736-42 https://www.ncbi.nlm.nih.gov/pubmed/19696760

369 Harvey J, Howell A, Morris J, Harvie M. Intermittent energy restriction for weight loss: Spontaneous reduction of energy intake on unrestricted days. Food Sci Nutr. 2018. 6:674-680 https://www.ncbi.nlm.nih.gov/pubmed/29876119

370 Harvie M, Wright C, Pegington M, McMullan D, Mitchell E, Martin B, Cutler RG, Evans G, Whiteside S, Maudsley S, Camandola S, Wang R, Carlson OD, Egan JM, Mattson MP, Howell A. The effect of intermittent energy and carbohydrate restriction $\mathrm{v}$. daily energy restriction on weight loss and metabolic disease risk markers in overweight women. Br J Nutr. 2013. 110:1534-47 https://www.ncbi.nlm.nih.gov/pubmed/23591120

371 Harvie MN, Sims AH, Pegington M, Spence K, Mitchell A, Vaughan AA, Allwood JW, Xu Y, Rattray NJ, Goodacre R, Evans DG, Mitchell E, McMullen D, Clarke RB, Howell A. Intermittent energy restriction induces changes in breast gene expression and systemic metabolism. Breast Cancer Res. 2016. 18:57 https://www.ncbi.nlm.nih.gov/pubmed/27233359

372 Hassan BK, Cunha DB, da Veiga GV, Pereira RA, Sichieri R. Changes in breakfast frequency and composition during adolescence: The Adolescent Nutritional Assessment Longitudinal Study, a cohort from Brazil. PLoS One. 2018. 13:e0200587 https://www.ncbi.nlm.nih.gov/pubmed/30024906 https://www.ncbi.nlm.nih.gov/pubmed/27335589 Profiles, Leptin, and Adiponectin in Overweight Subjects (BMI > 25). Int Cardiovasc Res J. 2014. 8:52-6 https://www.ncbi.nlm.nih.gov/pubmed/24936481 A chronophysiological approach. Chronobiol Int. 2016. 33:1018-36 https://www.ncbi.nlm.nih.gov/pubmed/27366928
Daily Eating Occasions Not

Reported, Size of Study Groups

Intervention/Exposure

Study Design

Daily Eating Occasions Not

Reported

Intervention/Exposure, Daily

Eating Occasions Not Reported

Daily Eating Occasions Not Reported

Study Design

Study Design

Daily Eating Occasions Not

Reported, Eating Frequency Data Collection

Intervention/Exposure 

variability, not sleep duration, is associated with caloric intake in adolescents. Sleep medicine. 2015;16(7):856-61. https://www.ncbi.nlm.nih.gov/pubmed/26002758.

377 He F, Zuo L, Emery W, Arciero P. High protein intermittent fasting increases serum polychlorinated biphenyls and decreases oxidative stress in obese adults. Journal of hypertension. 2017. 35:e258

$378 \quad$ Headland M, Clifton P, Keogh J. Intermittent compared to continuous energy restriction on weight loss and weight maintenance: effects after 12 months. Obesity research \& clinical practice. 2019. 13:268-269

379 Headland ML, Clifton PM, Keogh JB. Effect of intermittent compared to continuous energy restriction on weight loss and weight maintenance after 12 months in healthy overweight or obese adults. Int J Obes (Lond). 2018;43(10):2028-36. https://www.ncbi.nlm.nih.gov/pubmed/30470804.

$380 \quad$ Headland ML, Clifton PM, Keogh JB. Effect of Intermittent Energy Restriction on Flow Mediated Dilatation, a Measure of Endothelial Function: A Short Report. Int J Environ Res Public Health. 2018;15(6). https://www.ncbi.nlm.nih.gov/pubmed/29867034.

381 Heart failure. J Pract Nurs. 2008. 58:20-5 https://www.ncbi.nlm.nih.gov/pubmed/19260364

\section{Study Design}

382 Heden TD, Liu Y, Sims LJ, Whaley-Connell AT, Chockalingam A, Dellsperger KC, Kanaley JA. Meal frequency differentially alters postprandial triacylglycerol and insulin concentrations in obese women. Obesity (Silver Spring). 2013. 21:123-9 https://www.ncbi.nlm.nih.gov/pubmed/23505176

383 Heery E, Kelleher CC, Wall PG, McAuliffe FM. Prediction of gestational weight gain - a biopsychosocial model. Public Health Nutr. 2015. 18:1488-98 https://www.ncbi.nlm.nih.gov/pubmed/25171690

Publication Status

Publication Status

Daily Eating Occasions Not

Reported

Intervention/Exposure, Daily Eating Occasions Not Reported

Size of Study Groups

384 Heery E, Wall PG, Kelleher CC, McAuliffe FM. Effects of dietary restraint and weight gain attitudes on gestational weight gain. Appetite. 2016. 107:501-510 https://www.ncbi.nlm.nih.gov/pubmed/27545671

385 Heilbronn LK, Civitarese AE, Bogacka I, Smith SR, Hulver M, Ravussin E. Glucose tolerance and skeletal muscle gene expression in response to alternate day fasting. Obes Res. 2005. 13:574-81 https://www.ncbi.nlm.nih.gov/pubmed/15833943

$386 \quad$ Heilbronn LK, Smith SR, Martin CK, Anton SD, Ravussin E. Alternate-day fasting in nonobese subjects: effects on body weight, body composition, and energy metabolism. Am J Clin Nutr. 2005. 81:69-73 https://www.ncbi.nlm.nih.gov/pubmed/15640462

387 Helle C, Hillesund ER, Wills AK, Overby NC. Evaluation of an eHealth intervention aiming to promote healthy food habits from infancy -the Norwegian randomized controlled trial Early Food for Future Health. Int J Behav Nutr Phys Act. 2019. 16:1 https://www.ncbi.nlm.nih.gov/pubmed/30606197

Daily Eating Occasions Not Reported

Intervention/Exposure

Daily Eating Occasions Not Reported, Size of Study Groups

Daily Eating Occasions Not Reported, Size of Study Groups

Age: Intervention/Exposure 

diagnosed with preterm labor. J Nurs Scholarsh. 2010. 42:147-55 https://www.ncbi.nlm.nih.gov/pubmed/20618599

389 Heriseanu AI, Hay P, Touyz S. The short inventory of grazing (SIG): development and validation of a new brief

Intervention/Exposure measure of a common eating behaviour with a compulsive dimension. J Eat Disord. 2019. 7:4 https://www.ncbi.nlm.nih.gov/pubmed/30774954

$390 \quad$ Herman CP, van Strien T, Polivy J. Undereating or eliminating overeating?. Am Psychol. 2008. 63:202-3 https://www.ncbi.nlm.nih.gov/pubmed/18377113

391 Hermenegildo Y, Lopez-Garcia E, Garcia-Esquinas E, Perez-Tasigchana RF, Rodriguez-Artalejo F, GuallarCastillon P. Distribution of energy intake throughout the day and weight gain: a population-based cohort study in Spain. Br J Nutr. 2016. 115:2003-10 https://www.ncbi.nlm.nih.gov/pubmed/27044416

392 Heron KE, Scott SB, Sliwinski MJ, Smyth JM. Eating behaviors and negative affect in college women's everyday lives. Int J Eat Disord. 2014. 47:853-9 https://www.ncbi.nlm.nih.gov/pubmed/24797029

393 Herrmann TS, Siega-Riz AM, Hobel CJ, Aurora C, Dunkel-Schetter C. Prolonged periods without food intake during pregnancy increase risk for elevated maternal corticotropin-releasing hormone concentrations. Am J Obstet Gynecol. 2001. 185:403-12 https://www.ncbi.nlm.nih.gov/pubmed/11518900

394 Hibi M, Hari S, Yamaguchi T, Mitsui Y, Kondo S, Katashima M. Effect of Short-Term Increase in Meal Frequency on Glucose Metabolism in Individuals with Normal Glucose Tolerance or Impaired Fasting Glucose: A Randomized Crossover Clinical Trial. Nutrients. 2019;11(9). https://www.ncbi.nlm.nih.gov/pubmed/31489910. 395 Hibi M, Katashima M, Tokuyama K, Satoh M. Effect of meal frequency on 24-h blood glucose profiles assessed with
continuous glucose monitoring in subjects with normal and impaired fasting glucose tolerance. Diabetes. 2012. 61:A187

396 Hibi M, Kubota C, Mizuno T, Aritake S, Mitsui Y, Katashima M, Uchida S. Effect of shortened sleep on energy expenditure, core body temperature, and appetite: a human randomised crossover trial. Sci Rep. 2017. 7:39640 https://www.ncbi.nlm.nih.gov/pubmed/28071649

397 Hibi M, Masumoto A, Naito Y, Kiuchi K, Yoshimoto Y, Matsumoto M, Katashima M, Oka J, Ikemoto S. Nighttime snacking reduces whole body fat oxidation and increases LDL cholesterol in healthy young women. Am J Physiol Regul Integr Comp Physiol. 2013. 304:R94-r101 https://www.ncbi.nlm.nih.gov/pubmed/23174861

Intervention/Exposure

Intervention/Exposure

Intervention/Exposure

Intervention/Exposure, Daily Eating Occasions Not Reported

Size of Study Groups

Study Design, Publication Status

Comparator

Daily Eating Occasions Not Reported, Size of Study Groups

Outcome 
$401 \quad$ Hirschler V, Buzzano K, Erviti A, Ismael N, Silva S, Dalamon R. Overweight and lifestyle behaviors of low socioeconomic elementary school children in Buenos Aires. BMC Pediatr. 2009. 9:17

\section{Study Design} https://www.ncbi.nlm.nih.gov/pubmed/19239682 Hirsh SP, Pons M, Joyal SV, Swick AG. Avoiding holiday seasonal weight gain with nutrient-supported intermittent
energy restriction: a pilot study. J Nutr Sci. 2019. 8:e11 https://www.ncbi.nlm.nih.gov/pubmed/30931109

Daily Eating Occasions Not Reported

Study Design, Daily Eating Occasions Not Reported Ramadan on fetal Doppler parameters, maternal lipid levels and neonatal outcomes. J Matern Fetal Neonatal Med. 2012. 25:975-7 https://www.ncbi.nlm.nih.gov/pubmed/21740320

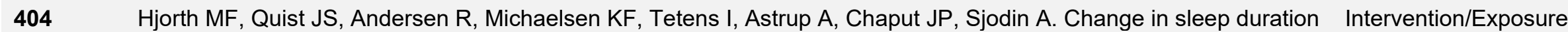
and proposed dietary risk factors for obesity in Danish school children. Pediatr Obes. 2014. 9:e156-9 https://www.ncbi.nlm.nih.gov/pubmed/25251317

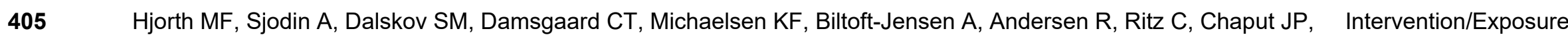
Astrup A. Sleep duration modifies effects of free ad libitum school meals on adiposity and blood pressure. Appl Physiol Nutr Metab. 2016. 41:33-40 https://www.ncbi.nlm.nih.gov/pubmed/26647154

406 Hoddy K, Kroeger C, Trepanowski J, Bhutani S, Barnosky A, Varady K. Meal timing during alternate day fasting: effects on body weight and coronary heart disease risk in obese adults. FASEB journal. 2014;28(Suppl. 1)

407 Hoddy KK, Bhutani S, Phillips SA, Varady KA. Effects of different degrees of insulin resistance on endothelial function in obese adults undergoing alternate day fasting. Nutr Healthy Aging. 2016. 4:63-71 https://www.ncbi.nlm.nih.gov/pubmed/28035343

408 Hoddy KK, Kroeger CM, Trepanowski JF, Barnosky A, Bhutani S, Varady KA. Meal timing during alternate day fasting: Impact on body weight and cardiovascular disease risk in obese adults. Obesity (Silver Spring). 2014. 22:2524-31 https://www.ncbi.nlm.nih.gov/pubmed/25251676

409 Hoddy KK, Kroeger CM, Trepanowski JF, Barnosky AR, Bhutani S, Varady KA. Safety of alternate day fasting and effect on disordered eating behaviors. Nutr J. 2015. 14:44 https://www.ncbi.nlm.nih.gov/pubmed/25943396
Study Design, Publication Status

Daily Eating Occasions Not Reported

Eating Frequency Data

Collection

Intervention/Exposure, Daily Eating Occasions Not Reported, Outcome 
$410 \quad$ Hoffmann DA, Carels RA. Does when you eat and exercise matter? Differences in eating and physical activity patterns in overweight and obese adults. Eat Weight Disord. 2016. 21:91-8

Intervention/Exposure https://www.ncbi.nlm.nih.gov/pubmed/26286898

411 Hofmann W, Adriaanse M, Vohs KD, Baumeister RF. Dieting and the self-control of eating in everyday environments: an experience sampling study. Br J Health Psychol. 2014. 19:523-39 https://www.ncbi.nlm.nih.gov/pubmed/23751109

412 Hojlund K, Wildner-Christensen M, Eshoj O, Skjaerbaek C, Holst JJ, Koldkjaer O, Moller Jensen D, Beck-Nielsen H. Reference intervals for glucose, beta-cell polypeptides, and counterregulatory factors during prolonged fasting. Am $J$ Physiol Endocrinol Metab. 2001. 280:E50-8 https://www.ncbi.nlm.nih.gov/pubmed/11120658

413 Holmback I, Ericson U, Gullberg B, Wirfalt E. Five meal patterns are differently associated with nutrient intakes, lifestyle factors and energy misreporting in a sub-sample of the Malmo Diet and Cancer cohort. Food Nutr Res. 2009; 53:1-16. https://www.ncbi.nlm.nih.gov/pubmed/19798420.

414 Holmback U, Lowden A, Akerfeldt T, Lennernas M, Hambraeus L, Forslund J, Akerstedt T, Stridsberg M, Forslund

414 A. The human body may buffer small differences in meal size and timing during a 24-h wake period provided energy balance is maintained. J Nutr. 2003. 133:2748-55 https://www.ncbi.nlm.nih.gov/pubmed/12949360

415 Holmback U. Metabolic, endocrine and mood responses to nocturnal eating in men are affected by sources of

dietary energy. Ups J Med Sci. 2002. 107:121-58 https://www.ncbi.nlm.nih.gov/pubmed/12696573

Intervention/Exposure, Daily Eating Occasions Not Reported

Study Design,

Intervention/Exposure, Daily Eating Occasions Not Reported

Study Design

Size of Study Groups

416 Holmstrup ME, Owens CM, Fairchild TJ, Kanaley JA. Effect of meal frequency on glucose and insulin excursions over the course of a day. e-SPEN. 2010. 5:e277-e280

417 Hopkins LC, Sattler M, Steeves EA, Jones-Smith JC, Gittelsohn J. Breakfast Consumption Frequency and Its Relationships to Overall Diet Quality, Using Healthy Eating Index 2010, and Body Mass Index among Adolescents in a Low-Income Urban Setting. Ecol Food Nutr. 2017. 56:297-311 https://www.ncbi.nlm.nih.gov/pubmed/28604287

418 Horne BD, May HT, Anderson JL, Kfoury AG, Bailey BM, McClure BS, Renlund DG, Lappe DL, Carlquist JF, Fisher PW, Pearson RR, Bair TL, Adams TD, Muhlestein JB. Usefulness of routine periodic fasting to lower risk of coronary artery disease in patients undergoing coronary angiography. Am J Cardiol. 2008. 102:814-819 https://www.ncbi.nlm.nih.gov/pubmed/18805103

419 Horne BD, Muhlestein JB, Lappe DL, May HT, Carlquist JF, Galenko O, Brunisholz KD, Anderson JL. Randomized cross-over trial of short-term water-only fasting: metabolic and cardiovascular consequences. Nutr Metab Cardiovasc Dis. 2013. 23:1050-7 https://www.ncbi.nlm.nih.gov/pubmed/23220077

Study Design

Size of Study Groups

Study Design, Daily Eating

Occasions Not Reported

Study Design, Daily Eating

Occasions Not Reported

Intervention/Exposure, Daily Eating Occasions Not Reported 
420 Horne BD, Muhlestein JB, May HT, Carlquist JF, Lappe DL, Bair TL, Anderson JL. Relation of routine, periodic fasting to risk of diabetes mellitus, and coronary artery disease in patients undergoing coronary angiography. $A m ~ J$ Cardiol. 2012. 109:1558-62 https://www.ncbi.nlm.nih.gov/pubmed/22425331

421 Horne BD. Is periodic fasting really good for reducing cardiovascular risk and improving heart health? Future Cardiology. 2011;7(6):721-4. https://www.ncbi.nlm.nih.gov/pubmed/22050055.

422 Horowitz JF, Coppack SW, Klein S. Whole-body and adipose tissue glucose metabolism in response to short-term fasting in lean and obese women. Am J Clin Nutr. 2001. 73:517-22 https://www.ncbi.nlm.nih.gov/pubmed/11237926

423 Huang TT, Howarth NC, Lin BH, Roberts SB, McCrory MA. Energy intake and meal portions: associations with BM percentile in U.S. children. Obes Res. 2004. 12:1875-85 https://www.ncbi.nlm.nih.gov/pubmed/15601985

$424 \quad$ Hughes S, Dennison CR. Progress in prevention: economic downturn = cardiovascular disease risk uptick?. $J$ Cardiovasc Nurs. 2009. 24:287-9 https://www.ncbi.nlm.nih.gov/pubmed/21206351

425

Hui A, Back L, Ludwig S, Gardiner P, Sevenhuysen G, Dean H, Sellers E, McGavock J, Morris M, Bruce S, etal. Lifestyle intervention on diet and exercise reduced excessive gestational weight gain in pregnant women under a randomized controlled trial. Obstetrical \& gynecological survey. 2012. 67:263-264

426 Hulman A, Faerch K, Vistisen D, Karsai J, Nyari TA, Tabak AG, Brunner EJ, Kivimaki M, Witte DR. Effect of time of day and fasting duration on measures of glycaemia: analysis from the Whitehall II Study. Diabetologia. 2013. 56:294-7 https://www.ncbi.nlm.nih.gov/pubmed/23143165 Hume DJ, Kroff J, Clamp LD, Lambert EV. Compensations for Weight Loss in Successful and Unsuccessful Dieters Am J Health Behav. 2015. 39:589-600 https://www.ncbi.nlm.nih.gov/pubmed/26248169

428 Huseinovic E, Winkvist A, Freisling H, Slimani N, Boeing H, Buckland G, Schwingshackl L, Olsen A, Tjonneland A, Stepien M, Boutron-Ruault MC, Mancini F, Artaud F, Kuhn T, Katzke V, Trichopoulou A, Naska A, Orfanos P, Tumino R, Masala G, Krogh V, Santucci de Magistris M, Ocke MC, Brustad M, Jensen TE, Skeie G, RodriguezBarranco M, Huerta JM, Ardanaz E, Quiros JR, Jakszyn P, Sonestedt E, Ericson U, Wennberg M, Key TJ, Aune D, Riboli E, Weiderpass E, Berteus Forslund H. Timing of eating across ten European countries - results from the European Prospective Investigation into Cancer and Nutrition (EPIC) calibration study. Public Health Nutr. 2019. 22:324-335 https://www.ncbi.nlm.nih.gov/pubmed/30326988
Intervention/Exposure, Daily

Eating Occasions Not Reported

Study Design, Publication Status

Intervention/Exposure, Daily

Eating Occasions Not Reported, Comparator

Study Design

Study Design

Study Design, Publication Status

Intervention/Exposure

Intervention/Exposure

Study Design

Intervention/Exposure, Daily Eating Occasions Not Reported, Size of Study Groups 
430 Hutchison AT, Liu B, Wittert GA, Heilbronn LK. Effects of intermittent fasting, with and without calorie restriction, on human metabolic health. Obesity reviews. 2016. 17:54

431 Hutchison AT, Liu B, Wood RE, Vincent AD, Thompson CH, O'Callaghan NJ, Wittert GA, Heilbronn LK. Effects of Intermittent Versus Continuous Energy Intakes on Insulin Sensitivity and Metabolic Risk in Women with Overweight. Obesity (Silver Spring). 2019. 27:50-58 https://www.ncbi.nlm.nih.gov/pubmed/30569640

432

Hutchison AT, Regmi P, Manoogian ENC, Fleischer JG, Wittert GA, Panda S, Heilbronn LK. Time-Restricted Feeding Improves Glucose Tolerance in Men at Risk for Type 2 Diabetes: A Randomized Crossover Trial. Obesity (Silver Spring). 2019. 27:724-732 https://www.ncbi.nlm.nih.gov/pubmed/31002478

433 Hyzyk AK, Reguła J, Jeszka J. Evaluation of total energy balance and food habits of obese children. Medycyna wieku rozwojowego. 2000;4(2):109-18. https://www.ncbi.nlm.nih.gov/pubmed/11013866.

434 Ibe Y, Miyakawa H, Fuse-Nagase Y, Hirose AS, Hirasawa R, Yachi Y, Fujihara K, Kobayashi K, Shimano H, Sone $\mathrm{H}$. Association of eating three meals irregularly with changes in BMI and weight among young Japanese men and women: A 2-year follow-up. Physiol Behav. 2016. 163:81-87 https://www.ncbi.nlm.nih.gov/pubmed/27126967

435 Ibrahim WH, Habib HM, Jarrar AH, Al Baz SA. Effect of Ramadan fasting on markers of oxidative stress and serum biochemical markers of cellular damage in healthy subjects. Ann Nutr Metab. 2008. 53:175-81 https://www.ncbi.nlm.nih.gov/pubmed/19011280

436 Ibrahim WH, Habib HM, Jarrar AH, Al Baz SA. Effect of Ramadan fasting on markers of oxidative stress and serum biochemical markers of cellular damage in healthy subjects. Ann Nutr Metab. 2008;53(3-4):175-81. https://www.ncbi.nlm.nih.gov/pubmed/19011280.

437 Ikeda N, Nishi N. First incidence and associated factors of overweight and obesity from preschool to primary school: longitudinal analysis of a national cohort in Japan. Int J Obes (Lond). 2019. 43:751-760 https://www.ncbi.nlm.nih.gov/pubmed/30659255

\section{8} Influences of breakfast on clock gen diabetes. Diabetes. 2017;66:A54 https://www.ncbi.nlm.nih.gov/pubmed/23263782
Publication Status

Daily Eating Occasions Not Reported, Size of Study Groups

Daily Eating Occasions Not

Reported, Comparator

\section{Language}

Daily Eating Occasions Not Reported

Daily Eating Occasions Not Reported

Daily Eating Occasions Not Reported

Daily Eating Occasions Not Reported

Publication Status

Daily Eating Occasions Not Reported

Study Design, Eating Frequency Data Collection 

northern Finland birth cohort 1986. PLoS One. 2013. 8:e73802 https://www.ncbi.nlm.nih.gov/pubmed/24040077

442 Jaaskelainen A, Schwab U, Kolehmainen M, Pirkola J, Jarvelin MR, Laitinen J. Associations of meal frequency and Metab Cardiovasc Dis. 2013. 23:1002-9 https://www.ncbi.nlm.nih.gov/pubmed/22901841

443 Jakubowicz D, Barnea M, Wainstein J, Froy O. High caloric intake at breakfast vs. dinner differentially influences weight loss of overweight and obese women. Obesity (Silver Spring). 2013. 21:2504-12 https://www.ncbi.nlm.nih.gov/pubmed/23512957

444 Jakubowicz D, Boaz M, Bar-Dayan Y, Wainstein J. Comparison of the effect of high calorie breakfast diet vs high calorie dinner diet on weight loss, ghrelin, lipids and appetite scores in obese non diabetic women. Endocrine reviews. 2012;33(12). 445 Jakubowicz D, Dayan YB, Wainstein J. The influence of meal timing on glucose metabolism and hyperandrogenism Health Status
in lean women with polycystic ovary syndrome. Diabetes. 2013. 62:A399 J46 Jakubowicz D, Wainstein J, Froy O. High-calorie breakfast improves weight loss and metabolism vs. isocaloric meal Publication Status
at dinner in obese women with metabolic syndrome. Diabetes. 2013. 62:A399

447 Jakubowicz D, Wainstein J, Landau Z, Raz I, Ahren B, Chapnik N, Ganz T, Menaged M, Barnea M, Bar-Dayan Y, etal. Influences of breakfast on circadian clock gene and AMPK mRNA expression and postprandial glycemia in healthy individuals and individuals with diabetes. Clinical obesity. 2018. 8:2-3 https://www.cochranelibrary.com/central/doi/10.1002/central/CN-01571505/full

448 Jakubowicz D, Wainstein J, Landau Z, Raz I, Ahren B, Chapnik N, Ganz T, Menaged M, Barnea M, Bar-Dayan Y, Froy O. Influences of Breakfast on Clock Gene Expression and Postprandial Glycemia in Healthy Individuals and Individuals With Diabetes: A Randomized Clinical Trial. Diabetes Care. 2017. 40:1573-1579 https://www.ncbi.nlm.nih.gov/pubmed/28830875

449 Jamshed H, Beyl RA, Della Manna DL, Yang ES, Ravussin E, Peterson CM. Early Time-Restricted Feeding Improves 24-Hour Glucose Levels and Affects Markers of the Circadian Clock, Aging, and Autophagy in Humans. Nutrients. 2019;11(6). https://www.ncbi.nlm.nih.gov/pubmed/31151228.

450 Jarrar AH, Beasley JM, Ohuma EO, Cheikh Ismail L, Qeshta DA, Mohamad MN, et al. Effect of High Fiber Cereal Intake on Satiety and Gastrointestinal Symptoms during Ramadan. Nutrients. 2019;11(4). https://www.ncbi.nlm.nih.gov/pubmed/31027300.

Publication Status

Daily Eating Occasions Not Reported

Daily Eating Occasions Not Reported, Comparator, Size of Study Groups

Intervention/Exposure Comparator 
451 Jebeile H, Gow ML, Lister N, Chisholm K, Grunseit A, Alexander S, Haghighi MM, Ayer J, Cowell CT, Baur L, etal. Intermittent energy restriction in adolescents with obesity: a pilot study. Obesity facts. 2018. 11:218-219

452 Jebeile H, Gow ML, Lister NB, Mosalman Haghighi M, Ayer J, Cowell CT, Baur LA, Garnett SP. Intermittent Energy Restriction Is a Feasible, Effective, and Acceptable Intervention to Treat Adolescents with Obesity. J Nutr. 2019. 149:1189-1197 https://www.ncbi.nlm.nih.gov/pubmed/31006807

453 Jeffery RW, Abbott G, Ball K, Crawford D. Behavior and weight correlates of weight-control efforts in Australian women living in disadvantage: The READI study. International journal of behavioral nutrition and physical activity. 2013;10. https://www.ncbi.nlm.nih.gov/pubmed/23621952.

\section{4} Jennings M. Snacking ourselves sick. HDA Now. 2012:25. https://www.ncbi.nlm.nih.gov/pubmed/22988698.

Publication Status

Daily Eating Occasions Not Reported

Intervention/Exposure, Daily Eating Occasions Not Reported

\section{Study Design,}

Intervention/Exposure,

Publication Status

455 Jensen MD, Ekberg K, Landau BR. Lipid metabolism during fasting. Am J Physiol Endocrinol Metab. 2001. 281:E789-93 https://www.ncbi.nlm.nih.gov/pubmed/11551856

456 Jensen ML, Corvalan C, Reyes M, Popkin BM, Taillie LS. Snacking patterns among Chilean children and adolescents: is there potential for improvement? Public Health Nutr. 2019:1-10. https://www.ncbi.nlm.nih.gov/pubmed/31124768.

457 Jimenez AM, Oliva SL, Vilar EG, De Cuevillas B, Morais Moreno MDC, Gabella De Prado J, Diaz EA, Mauro Martin IS. The Mediterranean diet pattern with intermittent semi-fasting may facilitate weight loss: randomised controlled trial. Mediterranean journal of nutrition and metabolism. 2019. 12:153-161

458 Johnson JB, Laub DR, John S. The effect on health of alternate day calorie restriction: eating less and more than needed on alternate days prolongs life. Med Hypotheses. 2006. 67:209-11 https://www.ncbi.nlm.nih.gov/pubmed/16529878

459 Johnson NA, Stannard SR, Rowlands DS, Chapman PG, Thompson CH, O'Connor H, Sachinwalla T, Thompson MW. Effect of short-term starvation versus high-fat diet on intramyocellular triglyceride accumulation and insulin resistance in physically fit men. Exp Physiol. 2006. 91:693-703 https://www.ncbi.nlm.nih.gov/pubmed/16627573

460 Johnstone AM, Faber P, Gibney ER, Elia M, Horgan G, Golden BE, Stubbs RJ. Effect of an acute fast on energy compensation and feeding behaviour in lean men and women. Int $J$ Obes Relat Metab Disord. 2002. 26:1623-8 https://www.ncbi.nlm.nih.gov/pubmed/12461679

Intervention/Exposure, Size of Study Groups

Study Design

Daily Eating Occasions Not Reported

Study Design

Intervention/Exposure, Size of Study Groups

Intervention/Exposure, Daily Eating Occasions Not Reported

Study Design 
462 Jurikova J, Julinkova S. Eating habits of students in Vyskov (The Czech Republic). Rocz Panstw Zakl Hig. 2007. 58:423-6 https://www.ncbi.nlm.nih.gov/pubmed/17929589

$463 \quad$ Kachurak A, Davey A, Bailey RL, Fisher JO. Daily Snacking Occasions and Weight Status Among US Children Aged 1 to 5 Years. Obesity (Silver Spring). 2018. 26:1034-1042 https://www.ncbi.nlm.nih.gov/pubmed/29656571

464 Kaczmarek JL, Musaad SM, Holscher HD. Time of day and eating behaviors are associated with the composition and function of the human gastrointestinal microbiota. Am J Clin Nutr. 2017. 106:1220-1231 https://www.ncbi.nlm.nih.gov/pubmed/28971851

$465 \quad$ Kahleova H, Lloren JI, Mashchak A, Hill M, Fraser GE. Meal Frequency and Timing Are Associated with Changes in Study Design Body Mass Index in Adventist Health Study 2. J Nutr. 2017. 147:1722-1728 https://www.ncbi.nlm.nih.gov/pubmed/28701389

\section{6}

Kajiyama S, Imai S, Hashimoto Y, Yamane C, Miyawaki T, Matsumoto S, Ozasa N, Tanaka M, Kajiyama S, Fukui M. Divided consumption of late-night-dinner improves glucose excursions in young healthy women: A randomized cross-over clinical trial. Diabetes Res Clin Pract. 2018. 136:78-84 https://www.ncbi.nlm.nih.gov/pubmed/29199002

467 Kakamu T, Hidaka T, Kumagai T, Masuishi Y, Kasuga H, Endo S, Sato S, Takeda A, Koizumi M, Fukushima T. Unhealthy changes in eating habits cause acute onset hypertension in the normotensive community-dwelling elderly-3 years cohort study. Medicine (Baltimore). 2019. 98:e15071 https://www.ncbi.nlm.nih.gov/pubmed/30985658

$468 \quad$ Kalam F, Kroeger CM, Trepanowski JF, Gabel K, Song JH, Cienfuegos S, Varady KA. Beverage intake during alternate-day fasting: Relationship to energy intake and body weight. Nutr Health. 2019. 25:167-171 https://www.ncbi.nlm.nih.gov/pubmed/30983506

469 Kanaley JA, Heden TD, Liu Y, Fairchild TJ. Alteration of postprandial glucose and insulin concentrations with meal frequency and composition. Br J Nutr. 2014. 112:1484-93 https://www.ncbi.nlm.nih.gov/pubmed/25231499

470 Kaneita Y, Uchiyama M, Yoshiike N, Ohida T. Associations of usual sleep duration with serum lipid and lipoprotein levels. Sleep. 2008;31(5):645-52. https://www.ncbi.nlm.nih.gov/pubmed/18517035

471 Kaneta T, Hakamatsuka T, Takanami K, Yamada T, Takase K, Sato A, Higano S, Kinomura S, Fukuda H, Takahashi S, Yamada S. Evaluation of the relationship between physiological FDG uptake in the heart and age, blood glucose level, fasting period, and hospitalization. Ann Nucl Med. 2006. 20:203-8 https://www.ncbi.nlm.nih.gov/pubmed/16715951 International Journal of Nursing Research. 2013. 17:317-328 
$473 \quad$ Karateke A, Kaplanoglu M, Avci F, Kurt RK, Baloglu A. The effect of ramadan fasting on fetal development. Pakistan journal of medical sciences. 2015;31(6):1295-9. https://www.ncbi.nlm.nih.gov/pubmed/26870085.

Daily Eating Occasions Not Reported, Outcome

474 Karfopoulou E, Brikou D, Mamalaki E, Bersimis F, Anastasiou CA, Hill JO, Yannakoulia M. Dietary patterns in weight Intervention/Exposure loss maintenance: results from the MedWeight study. Eur J Nutr. 2017. 56:991-1002 https://www.ncbi.nlm.nih.gov/pubmed/26744302

475 Karkkainen U, Mustelin L, Raevuori A, Kaprio J, Keski-Rahkonen A. Successful weight maintainers among young adults-A ten-year prospective population study. Eat Behav. 2018. 29:91-98 https://www.ncbi.nlm.nih.gov/pubmed/29549864

$476 \quad$ Karwacki-Marugg C, Huddy K, Bernstein B, Whitaker M, Pranitis L, Morse B, Colangelo R, Stuart M, O'Donnell M, Kelsey AM. Support for Women Achieving Cardiovascular Health Through Exercise And Nutrition (SWAN) Study Pilot. Conn Med. 2016. 80:69-74 https://www.ncbi.nlm.nih.gov/pubmed/27024976

$477 \quad$ Kassab S, Abdul-Ghaffar T, Nagalla DS, Sachdeva U, Nayar U. Interactions between leptin, neuropeptide-Y and insulin with chronic diurnal fasting during Ramadan. Ann Saudi Med. 2004. 24:345-9 https://www.ncbi.nlm.nih.gov/pubmed/15573845

$478 \quad$ Kassab SE, Abdul-Ghaffar T, Nagalla DS, Sachdeva U, Nayar U. Serum leptin and insulin levels during chronic diurnal fasting. Asia Pac J Clin Nutr. 2003. 12:483-7 https://www.ncbi.nlm.nih.gov/pubmed/14672875

Daily Eating Occasions Not Reported

Intervention/Exposure

Daily Eating Occasions Not

Reported

Intervention/Exposure, Daily Eating Occasions Not Reported

Study Design

Kaufman FR, Hirst K, Linder B, Baranowski T, Cooper DM, Foster GD, Goldberg L, Harrell JS, Marcus MD, Trevino RP. Risk factors for type 2 diabetes in a sixth- grade multiracial cohort: the HEALTHY study. Diabetes Care. 2009. 32:953-5 https://www.ncbi.nlm.nih.gov/pubmed/19196888

480 Kavehmanesh Z, Abolghasemi H. Maternal Ramadan fasting and neonatal health. J Perinatol. 2004. 24:748-50 Kavehmanesh Z, Abolghasemi H. Maternal Ramad
https://www.ncbi.nlm.nih.gov/pubmed/15343350 Kent LM, Worsley A. Breakfast size is related to body mass index for men, but not women. Nutr Res. 2010. 30:240-5 https://www.ncbi.nlm.nih.gov/pubmed/20534326

482 Keogh JB, Pedersen E, Petersen KS, Clifton PM. Effects of intermittent compared to continuous energy restriction on short-term weight loss and long-term weight loss maintenance. Clin Obes. 2014. 4:150-6 https://www.ncbi.nlm.nih.gov/pubmed/25826770

Kerr MA, Rennie KL, McCaffrey TA, Wallace JM, Hannon-Fletcher MP, Livingstone MB. Snacking patterns among adolescents: a comparison of type, frequency and portion size between Britain in 1997 and Northern Ireland in 2005. Br J Nutr. 2009. 101:122-31 https://www.ncbi.nlm.nih.gov/pubmed/18533071
Intervention/Exposure, Daily Eating Occasions Not Reported, Country

Study Design

Intervention/Exposure, Daily Eating Occasions Not Reported

Study Design, Outcome 
484 Keski-Rahkonen A, Bulik CM, Pietilainen KH, Rose RJ, Kaprio J, Rissanen A. Eating styles, overweight and obesity in young adult twins. Eur J Clin Nutr. 2007. 61:822-9 https://www.ncbi.nlm.nih.gov/pubmed/17251930

485 Keski-Rahkonen A, Kaprio J, Rissanen A, Virkkunen M, Rose RJ. Breakfast skipping and health-compromising behaviors in adolescents and adults. Eur J Clin Nutr. 2003. 57:842-53 https://www.ncbi.nlm.nih.gov/pubmed/12821884
Kessler CS, Stange R, Schlenkermann M, Jeitler M, Michalsen A, Selle A, Raucci F, Steckhan N. A nonrandomized controlled clinical pilot trial on 8 wk of intermittent fasting (24 h/wk). Nutrition. 2018. 46:143-152.e2 https://www.ncbi.nlm.nih.gov/pubmed/29031771

487 Khan N, Rasheed A, Ahmed H, Aslam F, Kanwal F. Effect of Ramadan fasting on glucose level, lipid profile, HbA1c and uric acid among medical students in Karachi, Pakistan. East Mediterr Health J. 2017. 23:274-279 https://www.ncbi.nlm.nih.gov/pubmed/28634977

\section{8}

Khochtali I, Hellara I, Chakroun T, Harzallah O, Dridi Z, Hamdi S, Hassine M, Nouira S. Effects of long term intermittent fasting on biochemical and homeostasis parameters (Ramadan research group prospective study). Diabetes. 2013. 62:A194

489 Khoshdel A, Kheiri S, Hashemi-Dehkordi E, Nasiri J, Shabanian-Borujeni S, Saedi E. The effect of Ramadan fasting on LH, FSH, oestrogen, progesterone and leptin in pregnant women. J Obstet Gynaecol. 2014. 34:634-8 https://www.ncbi.nlm.nih.gov/pubmed/24914688

490 Khoshdel A, Kheiri S, Nasiri J, Taheri E, Najafi M, Salehifard AZ, Jafari A. Comparison of growth parameters of infants of Ramadan fasted and non-fasted mothers. Iranian Journal of Endocrinology \& Metabolism. 2008. 10:188188

491 Kim J, Kang SW, Mallilankaraman K, Baik SH, Lim JC, Balaganapathy P, She DT, Lok KZ, Fann DY, Thambiayah U, Tang SC, Stranahan AM, Dheen ST, Gelderblom M, Seet RC, Karamyan VT, Vemuganti R, Sobey CG, Mattson MP, Jo DG, Arumugam TV. Transcriptome analysis reveals intermittent fasting-induced genetic changes in ischemic stroke. Hum Mol Genet. 2018. 27:2405 https://www.ncbi.nlm.nih.gov/pubmed/29688516

492 Kim KW, Song MY, Chung SH, Chung WS. Effect of modified fasting therapy on body weight, fat and muscle mass, and blood chemistry in patients with obesity. J Tradit Chin Med. 2016. 36:57-62 https://www.ncbi.nlm.nih.gov/pubmed/26946620

Kim S, DeRoo LA, Sandler DP. Eating patterns and nutritional characteristics ass
Health Nutr. 2011. 14:889-95 https://www.ncbi.nlm.nih.gov/pubmed/21029511
Daily Eating Occasions Not Reported

Study Design

Daily Eating Occasions Not Reported, Size of Study Groups

Daily Eating Occasions Not Reported, Country

Study Design, Publication Status

Intervention/Exposure, Daily Eating Occasions Not Reported

Study Design, Daily Eating

Occasions Not Reported,

Outcome

Publication Status

Study Design,

Intervention/Exposure, Daily

Eating Occasions Not Reported

Outcome

Publication Status energy restriction in obese males. Obesity facts. 2012. 5:34-35 


\section{Citation}

495

Kirkendall DT, Leiper JB, Bartagi Z, Dvorak J, Zerguini Y. The influence of Ramadan on physical performance measures in young Muslim footballers. Journal of sports sciences. 2008;26:S1527.https://www.ncbi.nlm.nih.gov/pubmed/19085449.

496 Kishel GF. Obesity in children. Minn Med. 2003.

Kito K, Kuriyama A, Takahashi Y, Nakayama T. Impacts of skipping breakfast and late dinner on the incidence of being overweight: a 3-year retrospective cohort study of men aged 20-49 years. J Hum Nutr Diet. 2019. 32:349-355 https://www.ncbi.nlm.nih.gov/pubmed/30821869

498 Kiyak Caglayan E, Engin-Ustun Y, Sari N, Gocmen AY, Polat MF. The effects of prolonged fasting on the levels of adiponectin, leptin, apelin, and omentin in pregnant women. J Obstet Gynaecol. 2016. 36:555-8 https://www.ncbi.nlm.nih.gov/pubmed/26759187

499 Kiyani MM, Memon AR, Amjad MI, Ameer MR, Sadiq M, Mahmood T. Study of Human Biochemical Parameters During and After Ramadan. J Relig Health. 2017. 56:55-62 https://www.ncbi.nlm.nih.gov/pubmed/26149778

\section{0} Kiziltan G, Karabudak E, Tuncay G, Avsar F, Tuncay P, Mungan O, Meral P. Dietary intake and nutritional status o Turkish pregnant women during Ramadan. Saudi Med J. 2005. 26:1782-7 https://www.ncbi.nlm.nih.gov/pubmed/16311666

$501 \quad$ Klempel MC, Bhutani S, Fitzgibbon M, Freels S, Varady KA. Dietary and physical activity adaptations to alternate day modified fasting: implications for optimal weight loss. Nutr J. 2010. 9:35 https://www.ncbi.nlm.nih.gov/pubmed/20815899

$502 \quad$ Klempel MC, Kroeger CM, Bhutani S, Trepanowski JF, Varady KA. Intermittent fasting combined with calorie restriction is effective for weight loss and cardio-protection in obese women. Nutr J. 2012. 11:98 https://www.ncbi.nlm.nih.gov/pubmed/23171320

$503 \quad$ Klempel MC, Kroeger CM, Norkeviciute E, Goslawski M, Phillips SA, Varady KA. Benefit of a low-fat over high-fat diet on vascular health during alternate day fasting. Nutr Diabetes. 2013. 3:e71 https://www.ncbi.nlm.nih.gov/pubmed/23712283

\section{4} Klempel MC, Kroeger CM, Varady KA. Alternate day fasting (ADF) with a high fat background diet produces similar weight loss and cardio-protection when compared to ADF with a low fat background diet. FASEB journal. $2012 ; 26$.
Klempel MC, Kroeger CM, Varady KA. Alternate day fasting (ADF) with a high-fat diet produces similar weight loss and cardio-protection as ADF with a low-fat diet. Metabolism. 2013. 62:137-43 https://www.ncbi.nlm.nih.gov/pubmed/22889512

\section{Rationale}

Daily Eating Occasions Not

Reported, Outcome

Study Design

Daily Eating Occasions Not

Reported, Eating Frequency

Data Collection

Daily Eating Occasions Not

Reported

Country

Country

Daily Eating Occasions Not

Reported

Daily Eating Occasions Not Reported, Comparator

Daily Eating Occasions Not

Reported, Comparator

Study Design, Publication Status

Intervention/Exposure

Comparator 


\section{6}

Klempel con

507

Klingenberg L, Chaput JP, Holmbäck U, Jennum P, Astrup A, Sjödin A. Sleep restriction is not associated with a positive energy balance in adolescent boys. American Journal of Clinical Nutrition. 2012. 96:240-248

508 Knutsson A, Karlsson B, Ornkloo K, Landstrom U, Lennernas M, Eriksson K. Postprandial responses of glucose, insulin and triglycerides: influence of the timing of meal intake during night work. Nutr Health. 2002. 16:133-41 https://www.ncbi.nlm.nih.gov/pubmed/12102366

509 Ko GT, Chan JC, Tong SD, Chan AW, Wong PT, Hui SS, Kwok R, Chan CL. Associations between dietary habits and risk factors for cardiovascular diseases in a Hong Kong Chinese working population-the "Better Health for Better Hong Kong" (BHBHK) health promotion campaign. Asia Pac J Clin Nutr. 2007. 16:757-65 https://www.ncbi.nlm.nih.gov/pubmed/18042539

$510 \quad$ Kobayashi F, Ogata H, Omi N, Nagasaka S, Yamaguchi S, Hibi M, Tokuyama K. Effect of breakfast skipping on diurnal variation of energy metabolism and blood glucose. Obes Res Clin Pract. 2014. 8:e201-98 https://www.ncbi.nlm.nih.gov/pubmed/24847666

511 Kobel S, Wirt T, Schreiber A, Kesztyüs D, Kettner S, Erkelenz N, Wartha O, Steinacker JM. Intervention effects of a school-based health promotion programme on obesity related behavioural outcomes. Journal of obesity. 2014. 2014:476230

512 Kolbert E. XXXL: why are we so fat? New Yorker. 2009:73-7. https://www.ncbi.nlm.nih.gov/pubmed/19697500.

Kolettis TM, Papathanasiou A, Tziallas D, Milionis HJ, Kastanioti CK, Achenbach K. Afternoon nap, meal ingestion
and circadian variation of acute myocardial infarction. International journal of cardiology. 2008;123(3):338-40.
https://www.ncbi.nlm.nih.gov/pubmed/17320206.

513 Kolettis TM, Papathanasiou A, Tziallas D, Milionis HJ, Kastanioti CK, Achenbach K. Afternoon nap, meal ingestic
and circadian variation of acute myocardial infarction. International journal of cardiology. 2008;123(3):338-40.
https://www.ncbi.nlm.nih.gov/pubmed/17320206.

513 Kolettis TM, Papathanasiou A, Tziallas D, Milionis HJ, Kastanioti CK, Achenbach K. Afternoon nap, meal ingestic
and circadian variation of acute myocardial infarction. International journal of cardiology. 2008;123(3):338-40.
https://www.ncbi.nlm.nih.gov/pubmed/17320206. Kong A, Beresford SA, Alfano CM, Foster-Schubert KE, Neuhouser ML, Johnson DB, Duggan C, Wang CY, Xiao L, Bain CE, McTiernan A. Associations between snacking and weight loss and nutrient intake among postmenopausal overweight to obese women in a dietary weight-loss intervention. J Am Diet Assoc. 2011. 111:1898-903 https://www.ncbi.nlm.nih.gov/pubmed/22117666 L, Jeffery RW, Bain CE, McTiernan A. Self-monitoring and eating-related behaviors are associated with 12-month weight loss in postmenopausal overweight-to-obese women. J Acad Nutr Diet. 2012. 112:1428-1435 https://www.ncbi.nlm.nih.gov/pubmed/22795495
Intervention/Exposure, Daily Eating Occasions Not Reported

Intervention/Exposure, Daily Eating Occasions Not Reported

Study Design, Comparator

Study Design,

Intervention/Exposure, Daily

Eating Occasions Not Reported

Comparator, Size of Study

Groups

Daily Eating Occasions Not Reported

Publication Status

Study Design

Study Design, Daily Eating

Occasions Not Reported

Intervention/Exposure, Daily Eating Occasions Not Reported 
$516 \quad$ Koopman KE, Caan MW, Nederveen AJ, Pels A, Ackermans MT, Fliers E, la Fleur SE, Serlie MJ. Hypercaloric diets with increased meal frequency, but not meal size, increase intrahepatic triglycerides: a randomized controlled trial.

Intervention/Exposure Hepatology. 2014. 60:545-53 https://www.ncbi.nlm.nih.gov/pubmed/24668862

517 Kordi R, Abdollahi M, Memari A-H, Najafabadi MG. Investigating Two Different Training Time Frames during Ramadan Fasting. Asian Journal of Sports Medicine. 2011;2(3):205-10.

Daily Eating Occasions Not https://www.ncbi.nlm.nih.gov/pubmed/22375240.

518 Kostecka M. The influence of preschool children's diets on the risk of lifestyle diseases. A pilot study. Rocz Panstw Zakl Hig. 2018. 69:139-145 https://www.ncbi.nlm.nih.gov/pubmed/29766692

519 Kosti RI, Panagiotakos DB, Mihas CC, Alevizos A, Zampelas A, Mariolis A, Tountas Y. Dietary habits, physical activity and prevalence of overweight/obesity among adolescents in Greece: the Vyronas study. Med Sci Monit. 2007. 13:Cr437-44 https://www.ncbi.nlm.nih.gov/pubmed/17901850

\begin{tabular}{lll}
\hline $\mathbf{5 2 0}$ & Kovtun K. Meal and sleep timing interact to affect total caloric intake. Sleep. 2016. 39:A55-A56 & Publication Status \\
\hline $\mathbf{5 2 1}$ & $\begin{array}{l}\text { Kral TV, Whiteford LM, Heo M, Faith MS. Effects of eating breakfast compared with skipping breakfast on ratings of } \\
\text { appetite and intake at subsequent meals in 8- to 10-y-old children. Am J Clin Nutr. 2011. 93:284-91 } \\
\text { https://www.ncbi.nlm.nih.gov/pubmed/21084650 }\end{array}$ & $\begin{array}{l}\text { Daily Eating Occasions Not } \\
\text { Reported, Outcome }\end{array}$ \\
\hline $\mathbf{5 2 2}$ & $\begin{array}{l}\text { Kral TVE, Chittams J, Moore RH. Relationship between food insecurity, child weight status, and parent-reported } \\
\text { child eating and snacking behaviors. J Spec Pediatr Nurs. 2017;22(2). } \\
\text { https://www.ncbi.nlm.nih.gov/pubmed/28321980. }\end{array}$ & Study Design \\
\hline $\mathbf{5 2 3}$ & $\begin{array}{l}\text { Kroeger C, Trapanowski J, Barnosky A, Klempel M, Varady K. Effect of 1 year of alternate day fasting versus daily } \\
\text { calorie restriction on type 2 diabetes risk. FASEB journal. 2015;29 }\end{array}$ & Study Design, Publication Status \\
\hline $\mathbf{5 2 4}$ & $\begin{array}{l}\text { Kroeger C, Trapanowski J, Klempel M, Bhutani S, Hoddy K, Varady K. Alternate day fasting is effective for weight } \\
\text { loss and weight maintenance in obese adults. FASEB journal. 2014;28S1 }\end{array}$ & Study Design, Publication Status \\
\hline $\mathbf{5 2 5}$ & $\begin{array}{l}\text { Kroeger CM, Klempel MC, Bhutani S, Trepanowski JF, Tangney CC, Varady KA. Improvement in coronary heart } \\
\text { disease risk factors during an intermittent fasting/calorie restriction regimen: Relationship to adipokine modulations. } \\
\text { Nutr Metab (Lond). 2012. 9:98 https://www.ncbi.nlm.nih.gov/pubmed/23113919 }\end{array}$ & Study Design, Daily Eating \\
\hline $\mathbf{5 2 6}$ & $\begin{array}{l}\text { Kroeger CM, Klempel MC, Bhutani S, Trepanowski JF, Varady KA. Improvement in coronary heart disease risk } \\
\text { factors during an intermittent fasting/calorie restriction regimen: relationship to adipokine modulations. FASEB } \\
\text { journal. 2013. 27(12). }\end{array}$ \\
\hline
\end{tabular}




\section{Citation}

527 Kroeger CM, Trepanowski JF, Klempel MC, Barnosky A, Bhutani S, Gabel K, Varady KA. Eating behavior traits of successful weight losers during 12 months of alternate-day fasting: An exploratory analysis of a randomized controlled trial. Nutr Health. 2018. 24:5-10 https://www.ncbi.nlm.nih.gov/pubmed/29353535

528 Kubota Y, Iso H, Sawada N, Tsugane S. Association of Breakfast Intake With Incident Stroke and Coronary Heart Disease: The Japan Public Health Center-Based Study. Stroke. 2016. 47:477-81 https://www.ncbi.nlm.nih.gov/pubmed/26732562

529 Kudo A, Asahi K, Satoh H, Iseki K, Moriyama T, Yamagata K, Tsuruya K, Fujimoto S, Narita I, Konta T, Kondo M, Shibagaki Y, Kasahara M, Watanabe T, Shimabukuro M. Fast eating is a strong risk factor for new-onset diabetes among the Japanese general population. Sci Rep. 2019. 9:8210 https://www.ncbi.nlm.nih.gov/pubmed/31160664

$530 \quad$ Kullen C, Prvan T, O'Connor H. Barriers and Enablers Influencing Dietary Practices in Australian Army Personnel Mil Med. 2019. 184:e213-e221 https://www.ncbi.nlm.nih.gov/pubmed/30371876

$531 \quad$ Kupers LK, de Pijper JJ, Sauer PJ, Stolk RP, Corpeleijn E. Skipping breakfast and overweight in 2- and 5-year-old Dutch children-the GECKO Drenthe cohort. Int J Obes (Lond). 2014. 38:569-71 https://www.ncbi.nlm.nih.gov/pubmed/24158122

532 Lam BCC, Han JSY, Ho CY, Teoh H, Yum MPS, Wong MTK, Koh GCH. The effect of intermittent energy restriction using meal replacements in overweight chinese subjects: a pilot randomized control trial. Obesity facts. 2018. 11:307

533 Lamine F, Bouguerra R, Jabrane J, Marrakchi Z, Ben Rayana MC, Ben Slama C, Gaigi S. Food intake and high density lipoprotein cholesterol levels changes during ramadan fasting in healthy young subjects. Tunis Med. 2006. 84:647-50 https://www.ncbi.nlm.nih.gov/pubmed/17193859 Lamri-Senhadji MY, El Kebir B, Belleville J, Bouchenak M. Assessment of dietary consumption and time-course of changes in serum lipids and lipoproteins before, during and after Ramadan in young Algerian adults. Singapore Med J. 2009. 50:288-94 https://www.ncbi.nlm.nih.gov/pubmed/19352573

535 Langlet B, Tang Bach M, Odegi D, Fagerberg P, loakimidis I. The Effect of Food Unit Sizes and Meal Serving Occasions on Eating Behaviour Characteristics: Within Person Randomised Crossover Studies on Healthy Women. Nutrients. 2018;10(7). https://www.ncbi.nlm.nih.gov/pubmed/29986529.

\section{Rationale}

Intervention/Exposure, Daily

Eating Occasions Not Reported

Daily Eating Occasions Not Reported

Daily Eating Occasions Not

Reported, Eating Frequency Data Collection

Study Design

Study Design

Publication Status

Daily Eating Occasions Not

Reported

Intervention/Exposure, Daily Eating Occasions Not Reported

Intervention/Exposure, Daily Eating Occasions Not Reported

Intervention/Exposure, Outcome Size of Study Groups 

Associated with Lower Long-Term Gains in Body Mass Index and Fat Mass in Middle-Aged Men and Women. $J$

Collection Nutr. 2019. 149:824-830 https://www.ncbi.nlm.nih.gov/pubmed/31034009

538 Larson N, Chen Y, Wall M, Winkler MR, Goldschmidt AB, Neumark-Sztainer D. Personal, behavioral, and environmental predictors of healthy weight maintenance during the transition to adulthood. Prev Med. 2018. 113:8090 https://www.ncbi.nlm.nih.gov/pubmed/29727637

$539 \quad$ Larson NI, Neumark-Sztainer D, Story M. Weight control behaviors and dietary intake among adolescents and young adults: longitudinal findings from Project EAT. J Am Diet Assoc. 2009. 109:1869-77 https://www.ncbi.nlm.nih.gov/pubmed/19857628

\section{0 Larsson I, Hulthen L, Landen M, Palsson E, Janson P, Stener-Victorin E. Dietary intake, resting energy expenditure, and eating behavior in women with and without polycystic ovary syndrome. Clin Nutr. 2016. 35:213-8 https://www.ncbi.nlm.nih.gov/pubmed/25743212

541 Laska MN, Lytle LA, Nanney MS, Moe SG, Linde JA, Hannan PJ. Results of a 2-year randomized, controlled obesity prevention trial: Effects on diet, activity and sleep behaviors in an at-risk young adult population. Prev Med. 2016. 89:230-236 https://www.ncbi.nlm.nih.gov/pubmed/27283096

542 Laska MN, Murray DM, Lytle LA, Harnack LJ. Longitudinal associations between key dietary behaviors and weight gain over time: transitions through the adolescent years. Obesity (Silver Spring). 2012. 20:118-25

Intervention/Exposure, Daily Eating Occasions Not Reported https://www.ncbi.nlm.nih.gov/pubmed/21701567

543 Latiri I, Sandid S, Fennani MA, Hadrich M, Masmoudi T, Maatoug C, Zammit-Chatti M, Chamari K, Ben Saad H. The Effects of Ramadan Fasting on the Spirometric Data of Healthy Adult Males. Am J Mens Health. 2017. 11:12141223 https://www.ncbi.nlm.nih.gov/pubmed/28625120

544 Lavizzo-Mourey R. The adolescent obesity epidemic. J Adolesc Health. 2009. 45:S6-7 https://www.ncbi.nlm.nih.gov/pubmed/19699438

545 Laxer RE, Brownson RC, Dubin JA, Cooke M, Chaurasia A, Leatherdale ST. Clustering of risk-related modifiable behaviours and their association with overweight and obesity among a large sample of youth in the COMPASS study. BMC Public Health. 2017. 17:102 https://www.ncbi.nlm.nih.gov/pubmed/28109270

$546 \quad$ Lazzeri G, Giallombardo D, Guidoni C, Zani A, Casorelli A, Grasso A, Pozzi T, Rossi S, Giacchi M. Nutritional surveillance in Tuscany: eating habits at breakfast, mid-morning and afternoon snacks among 8-9 y-old children. $J$ Prev Med Hyg. 2006. 47:91-9 https://www.ncbi.nlm.nih.gov/pubmed/17217185

547 Leahy K, Berlin KS, Banks GG, Bachman J. The Relationship Between Intuitive Eating and Postpartum Weight Loss. Matern Child Health J. 2017. 21:1591-1597 https://www.ncbi.nlm.nih.gov/pubmed/28176035

Daily Eating Occasions Not Reported

Daily Eating Occasions Not Reported

Daily Eating Occasions Not Reported, Outcome

\section{Study Design}

Study Design

Study Design

Study Design, Intervention/Exposure 


\section{8} Leblanc inte

549 LeCheminant GM, LeCheminant JD, Tucker LA, Bailey BW. A randomized controlled trial to study the effects of
breakfast on energy intake, physical activity, and body fat in women who are nonhabitual breakfast eaters. Appetite. 2017. 112:44-51 https://www.ncbi.nlm.nih.gov/pubmed/28063876

$550 \quad$ LeCheminant JD, Christenson E, Bailey BW, Tucker LA. Restricting night-time eating reduces daily energy intake in healthy young men: a short-term cross-over study. Br J Nutr. 2013. 110:2108-13 https://www.ncbi.nlm.nih.gov/pubmed/23702187

551 LeCroy MN, Truesdale KP, Matheson DM, Karp SM, Moore SM, Robinson TN, et al. Snacking characteristics and patterns and their associations with diet quality and BMI in the Childhood Obesity Prevention and Treatment Research Consortium. Public Health Nutr. 2019:1-11. https://www.ncbi.nlm.nih.gov/pubmed/31112114.

552 Lee EY, Kang B, Yang Y, Yang HK, Kim HS, Lim SY, Lee JH, Lee SS, Suh BK, Yoon KH. Study Time after School and Habitual Eating Are Associated with Risk for Obesity among Overweight Korean Children: A Prospective Study. Obes Facts. 2018. 11:46-55 https://www.ncbi.nlm.nih.gov/pubmed/29408816

553 Lee SK. Acculturation, meal frequency, eating-out, and body weight in Korean Americans. Nutr Res Pract. 2008. 2:269-74 https://www.ncbi.nlm.nih.gov/pubmed/20016729

554 Lee TS, Kim JS, Hwang YJ, Park YC. Habit of Eating Breakfast Is Associated with a Lower Risk of Hypertension. $J$ Lifestyle Med. 2016. 6:64-67 https://www.ncbi.nlm.nih.gov/pubmed/27924285

555 Leech RM, Timperio A, Worsley A, McNaughton SA. Eating patterns of Australian adults: associations with blood pressure and hypertension prevalence. Eur J Nutr. 2019. 58:1899-1909 https://www.ncbi.nlm.nih.gov/pubmed/29876653

556 Leech RM, Worsley A, Timperio A, McNaughton SA. The role of energy intake and energy misreporting in the associations between eating patterns and adiposity. Eur J Clin Nutr. 2018. 72:142-147 https://www.ncbi.nlm.nih.gov/pubmed/28561038

557 Leidy HJ, Armstrong CL, Tang M, Mattes RD, Campbell WW. The influence of higher protein intake and greater eating frequency on appetite control in overweight and obese men. Obesity (Silver Spring). 2010. 18:1725-32 https://www.ncbi.nlm.nih.gov/pubmed/20339363
Leidy HJ, Hoertel HA, Douglas SM, Higgins KA, Shafer RS. A high-protein breakfast prevents body fat gain, through reductions in daily intake and hunger, in "Breakfast skipping" adolescents. Obesity (Silver Spring). 2015. 23:1761-4 https://www.ncbi.nlm.nih.gov/pubmed/26239831
Publication Status

Daily Eating Occasions Not Reported

Intervention/Exposure, Daily Eating Occasions Not Reported

Study Design, Daily Eating Occasions Not Reported

Daily Eating Occasions Not

Reported, Eating Frequency Data Collection

Study Design

Study Design

Study Design

Study Design

Eating Frequency Data Collection

Daily Eating Occasions Not Reported, Comparator 
559 Leidy HJ, Hoertel HA, Douglas SM, Shafer RS. Daily addition of a protein-rich breakfast for long-term improvements in energy intake regulation and body weight management in overweight \& obese 'breakfast skipping' young people.

Study Design, Publication Status FASEB journal. 2013;27.

560 Leidy HJ, Racki EM. The addition of a protein-rich breakfast and its effects on acute appetite control and food intake in 'breakfast-skipping' adolescents. Int J Obes (Lond). 2010. 34:1125-33 https://www.ncbi.nlm.nih.gov/pubmed/20125103

561 Leidy HJ, Tang M, Armstrong CL, Martin CB, Campbell WW. The effects of consuming frequent, higher protein meals on appetite and satiety during weight loss in overweight/obese men. Obesity (Silver Spring). 2011. 19:818-24 https://www.ncbi.nlm.nih.gov/pubmed/20847729

562 Leiper JB, Maughan RJ, Kirkendall DT, Bartagi Z, Zerguini Y, Junge A, et al. The F-MARC study on Ramadan and football: research design, population, and environmental conditions. J Sports Sci. 2008;26 Suppl 3:S7-13. https://www.ncbi.nlm.nih.gov/pubmed/19085448.

563 Leiper JB, Watson P, Evans G, Dvorak J. Intensity of a training session during Ramadan in fasting and non-fasting Tunisian youth football players. Journal of Sports Sciences. 2008. 26:S71-9. https://www.ncbi.nlm.nih.gov/pubmed/19085454

564 Lemmens SG, Martens EA, Born JM, Martens MJ, Westerterp-Plantenga MS. Staggered meal consumption facilitates appetite control without affecting postprandial energy intake. J Nutr. 2011. 141:482-8 https://www.ncbi.nlm.nih.gov/pubmed/21270370

565 Leong SL, Gray A, Haszard J, Horwath C. Weight-Control Methods, 3-Year Weight Change, and Eating Behaviors: A Prospective Nationwide Study of Middle-Aged New Zealand Women. J Acad Nutr Diet. 2016. 116:1276-84 https://www.ncbi.nlm.nih.gov/pubmed/27061782

566 Lessan N, Saadane I, Alkaf B, Hambly C, Buckley AJ, Finer N, Speakman JR, Barakat MT. The effects of Ramadan fasting on activity and energy expenditure. Am J Clin Nutr. 2018. 107:54-61 https://www.ncbi.nlm.nih.gov/pubmed/29381798

Daily Eating Occasions Not Reported, Outcome

Outcome, Size of Study Groups

Study Design, Publication Status

Daily Eating Occasions Not Reported

Daily Eating Occasions Not Reported, Outcome

Intervention/Exposure Lesser LI, Cohen DA, Brook RH. Changing eating habits for the medical profession. Jama. 2012. 308:983-4

$\begin{array}{ll}567 & \text { Lesser LI, Cohen DA, Brook RH. Changing eating } \\ \text { https://www.ncbi.nlm.nih.gov/pubmed/22968886 }\end{array}$ consumption. Journal of Parenteral and Enteral Nutrition. 2014;38(2):263-8. https://www.ncbi.nlm.nih.gov/pubmed/23599248.

Daily Eating Occasions Not

Reported

Publication Status

Intervention/Exposure 

index meal: A crossover trial in healthy volunteers. Clin Nutr. 2019. 38:465-471 https://www.ncbi.nlm.nih.gov/pubmed/29248250

570 Levitsky DA, Halbmaier CA, Mrdjenovic G. The freshman weight gain: a model for the study of the epidemic of obesity. Int J Obes Relat Metab Disord. 2004. 28:1435-42 https://www.ncbi.nlm.nih.gov/pubmed/15365585

Daily Eating Occasions Not Reported

571 Levitsky DA, Raea Limb JE, Wilkinson L, Sewall A, Zhong Y, Olabi A, Hunter J. Lack of negative autocorrelations of daily food intake on successive days challenges the concept of the regulation of body weight in humans. Appetite. 2017. 116:277-283 https://www.ncbi.nlm.nih.gov/pubmed/28483583

572 Li C, Ostermann T, Hardt M, Ludtke R, Broecker-Preuss M, Dobos G, Michalsen A. Metabolic and psychological response to 7-day fasting in obese patients with and without metabolic syndrome. Forsch Komplementmed. 2013. 20:413-20 https://www.ncbi.nlm.nih.gov/pubmed/24434755

573 Li G, Xie C, Lu S, Nichols RG, Tian Y, Li L, Patel D, Ma Y, Brocker CN, Yan T, Krausz KW, Xiang R, Gavrilova O, Patterson AD, Gonzalez FJ. Intermittent Fasting Promotes White Adipose Browning and Decreases Obesity by Shaping the Gut Microbiota. Cell Metab. 2017. 26:801 https://www.ncbi.nlm.nih.gov/pubmed/29117546

574 Li LB, Wang N, Wu XL, Wang L, Li JJ, Yang M, Ma J. Energy Balance-related Behaviors Are Related to Cardiometabolic Parameters and Predict Adiposity in 8-14-year-old Overweight Chinese Children One Year Later. Biomed Environ Sci. 2016. 29:754-757 https://www.ncbi.nlm.nih.gov/pubmed/27927275

575 Lindvall K, Jenkins P, Scribani M, Emmelin M, Larsson C, Norberg M, Weinehall L. Comparisons of weight change, eating habits and physical activity between women in Northern Sweden and Rural New York State- results from a longitudinal study. Nutr J. 2015. 14:88 https://www.ncbi.nlm.nih.gov/pubmed/26319494

576 Lipsky LM, Haynie DL, Liu D, Chaurasia A, Gee B, Li K, lannotti RJ, Simons-Morton B. Trajectories of eating behaviors in a nationally representative cohort of U.S. adolescents during the transition to young adulthood. Int $J$ Behav Nutr Phys Act. 2015. 12:138 https://www.ncbi.nlm.nih.gov/pubmed/26537771

Intervention/Exposure, Daily Eating Occasions Not Reported

Intervention/Exposure, Daily

Eating Occasions Not Reported

Study Design, Publication Status, Non-human

Intervention/Exposure

Intervention/Exposure

Intervention/Exposure, Daily Eating Occasions Not Reported

577 Liu B, Hutchison AT, Thompson CH, Lange K, Heilbronn LK. Markers of adipose tissue inflammation are transiently elevated during intermittent fasting in women who are overweight or obese. Obes Res Clin Pract. 2019. 13:408-415 https://www.ncbi.nlm.nih.gov/pubmed/31302012

578 Liu B, Hutchison AT, Thompson CH, Wittert GA, Heilbronn L. Adipose tissue remodeling following eight-week calorie restriction or intermittent fasting in females who are overweight and obese. Diabetes. 2017. 66:A550 
580 Lofthou 2:8-9

581 Lojko D, Stelmach-Mardas M, Suwalska A. Diet quality and eating patterns in euthymic bipolar patients. Eur Rev Med Pharmacol Sci. 2019. 23:1221-1238 https://www.ncbi.nlm.nih.gov/pubmed/30779092

582 Lombardo M, Bellia A, Padua E, Annino G, Guglielmi V, D’Adamo M, lellamo F, Sbraccia P. Morning meal more efficient for fat loss in a 3-month lifestyle intervention. J Am Coll Nutr. 2014. 33:198-205 https://www.ncbi.nlm.nih.gov/pubmed/24809437

583 Lopez Minguez J, Saxena R, Bandin Saura C, Scheer F, Garaulet Aza M. Dinner timing interacts with MTNR1B SNP to influence glucose tolerance in natural late eaters. Sleep. 2016. 39:A51-A52

584 Lopez-Bueno M, Gonzalez-Jimenez E, Navarro-Prado S, Montero-Alonso MA, Schmidt-RioValle J. Influence of age and religious fasting on the body composition of Muslim women living in a westernized context. Nutr Hosp. 2014 31:1067-73 https://www.ncbi.nlm.nih.gov/pubmed/25726194

585 López-Bueno M, González-Jiménez E, Navarro-Prado S, Montero-Alonso MA, Schmidt-RioValle J. Influence of age and religious fasting on the body composition of Muslim women living in a westernized context. Nutrición hospitalaria. 2015. 31:1067-1073

$586 \quad$ Lopez-Minguez J, Saxena R, Bandin C, Scheer FA, Garaulet M. Late dinner impairs glucose tolerance in MTNR1B risk allele carriers: A randomized, cross-over study. Clin Nutr. 2018. 37:1133-1140 https://www.ncbi.nlm.nih.gov/pubmed/28455106

587 Lopez-Olmedo N, Hernandez-Cordero S, Neufeld LM, Garcia-Guerra A, Mejia-Rodriguez F, Mendez GomezHumaran I. The Associations of Maternal Weight Change with Breastfeeding, Diet and Physical Activity During the Postpartum Period. Matern Child Health J. 2016. 20:270-80 https://www.ncbi.nlm.nih.gov/pubmed/26525558

588 Loy SL, Cheng TS, Colega MT, Cheung YB, Godfrey KM, Gluckman PD, Kwek K, Saw SM, Chong YS, Padmapriya N, Muller-Riemenschneider F, Lek N, Yap F, Chong MF, Chan JKY. Predominantly night-time feeding and maternal glycaemic levels during pregnancy. Br J Nutr. 2016. 115:1563-1570 https://www.ncbi.nlm.nih.gov/pubmed/26949026

589 Lucassen EA, Zhao X, Rother KI, Mattingly MS, Courville AB, de Jonge L, Csako G, Cizza G. Evening chronotype is associated with changes in eating behavior, more sleep apnea, and increased stress hormones in short sleeping obese individuals. PLoS One. 2013. 8:e56519 https://www.ncbi.nlm.nih.gov/pubmed/23483886

Maccario M, Aimaretti G, Corneli G, Gauna C, Grottoli S, Bidlingmaier M, Strasburger CJ, Dieguez C, Casanueva FF, Ghigo E. Short-term fasting abolishes the sex-related difference in $\mathrm{GH}$ and leptin secretion in humans. Am J Physiol Endocrinol Metab. 2000. 279:E411-6 https://www.ncbi.nlm.nih.gov/pubmed/10913042
Publication Status

Study Design

Comparator, Size of Study Groups

Study Design, Publication Status

Intervention/Exposure, Daily

Eating Occasions Not Reported

Daily Eating Occasions Not Reported

Intervention/Exposure, Daily Eating Occasions Not Reported, Comparator

Intervention/Exposure

Daily Eating Occasions Not Reported

Daily Eating Occasions Not Reported

Study Design,

Intervention/Exposure, Daily Eating Occasions Not Reported, Comparator 
592 MacFarlane A, Cleland V, Crawford D, Campbell K, Timperio A. Longitudinal examination of the family food environment and weight status among children. Int J Pediatr Obes. 2009. 4:343-52 https://www.ncbi.nlm.nih.gov/pubmed/19922051

593 Madjd A, Taylor MA, Delavari A, Malekzadeh R, Macdonald IA, Farshchi HR. Beneficial effect of high energy intake at lunch rather than dinner on weight loss in healthy obese women in a weight-loss program: a randomized clinical trial. Am J Clin Nutr. 2016. 104:982-989 https://www.ncbi.nlm.nih.gov/pubmed/27581472

594 Madkour MI, El-Serafi A T, Jahrami HA, Sherif NM, Hassan RE, Awadallah S, Faris MAE. Ramadan diurnal intermittent fasting modulates SOD2, TFAM, Nrf2, and sirtuins (SIRT1, SIRT3) gene expressions in subjects with overweight and obesity. Diabetes Res Clin Pract. 2019. 155:107801 https://www.ncbi.nlm.nih.gov/pubmed/31356832

$595 \quad$ Maffeis C, Provera S, Filippi L, Sidoti G, Schena S, Pinelli L, Tato L. Distribution of food intake as a risk factor for childhood obesity. Int J Obes Relat Metab Disord. 2000. 24:75-80 https://www.ncbi.nlm.nih.gov/pubmed/10702754

Daily Eating Occasions Not Reported

Intervention/Exposure, Daily Eating Occasions Not Reported

Daily Eating Occasions Not Reported, Eating Frequency Data Collection

\section{Study Design}

Study Design, Publication Status

596 Makarem N, Aggarwal B, Sears DD, St-Onge MP, Castaneda SF, Talavera GA, Marinac CR, Patterson R, SotresAlvarez D, Garcia ML, etal. Circadian timing of food intake is associated with cardiometabolic risk in us hispanics/latinos: results from the hispanic community health study/study of latinos. Circulation. 2018. 138(6 ).

597 Makvandi S, Karimi L, Mahdavian M, Bastami A. No Differences in Hematological Parameters of Fasting and NonFasting Pregnant Women Three Months after Ramadan. Int J Vitam Nutr Res. 2018. 88:258-262 https://www.ncbi.nlm.nih.gov/pubmed/31124419

598 Malinauskas BM, Raedeke TD, Aeby VG, Smith JL, Dallas MB. Dieting practices, weight perceptions, and body composition: a comparison of normal weight, overweight, and obese college females. Nutr J. 2006. 5:11 https://www.ncbi.nlm.nih.gov/pubmed/16579846

$599 \quad$ Marinac CR, Sears DD, Natarajan L, Gallo LC, Breen Cl, Patterson RE. Frequency and Circadian Timing of Eating May Influence Biomarkers of Inflammation and Insulin Resistance Associated with Breast Cancer Risk. PLoS One. 2015. 10:e0136240 https://www.ncbi.nlm.nih.gov/pubmed/26305095

600 Marlatt KL, Farbakhsh K, Dengel DR, Lytle LA. Breakfast and fast food consumption are associated with selected biomarkers in adolescents. Prev Med Rep. 2016. 3:49-52 https://www.ncbi.nlm.nih.gov/pubmed/26844187 
602 Marmonier C, Chapelot D, Fantino M, Louis-Sylvestre J. Snacks consumed in a nonhungry state have poor satiating efficiency: influence of snack composition on substrate utilization and hunger. American journal of clinical nutrition. 2002;76(3):518-28. https://www.ncbi.nlm.nih.gov/pubmed/12197994

603 Martin J, MacDonald-Wicks L, Hure A, Smith R, Collins CE. Reducing postpartum weight retention and improving breastfeeding outcomes in overweight women: a pilot randomised controlled trial. Nutrients. 2015. 7:1464-79 https://www.ncbi.nlm.nih.gov/pubmed/25723973

604 Masheb RM, Grilo CM. Eating patterns and breakfast consumption in obese patients with binge eating disorder. Behav Res Ther. 2006. 44:1545-53 https://www.ncbi.nlm.nih.gov/pubmed/16376851

605 Mathew S, Krug S, Skurk T, Halama A, Stank A, Artati A, Prehn C, Malek JA, Kastenmuller G, Romisch-Margl W, Adamski J, Hauner H, Suhre K. Metabolomics of Ramadan fasting: an opportunity for the controlled study of physiological responses to food intake. J Transl Med. 2014. 12:161 https://www.ncbi.nlm.nih.gov/pubmed/24906381

606 Mattson MP. The need for controlled studies of the effects of meal frequency on health. Lancet. 2005. 365:1978-80 https://www.ncbi.nlm.nih.gov/pubmed/15936428

607 Maughan RJ, Bartagi Z, Dvorak J, Zerguini Y. Dietary intake and body composition of football players during the holy month of Ramadan. J Sports Sci. 2008. 26 Suppl 3:S29-38 https://www.ncbi.nlm.nih.gov/pubmed/19085450

608 Maukonen M, Kanerva N, Partonen T, Mannisto S. Chronotype and energy intake timing in relation to changes in anthropometrics: a 7-year follow-up study in adults. Chronobiol Int. 2019. 36:27-41 https://www.ncbi.nlm.nih.gov/pubmed/30212231

609 Mazurak N, Gunther A, Grau FS, Muth ER, Pustovoyt M, Bischoff SC, Zipfel S, Enck P. Effects of a 48-h fast on heart rate variability and cortisol levels in healthy female subjects. Eur J Clin Nutr. 2013. 67:401-6 https://www.ncbi.nlm.nih.gov/pubmed/23403876

610 McCaffree J. Childhood eating patterns: the roles parents play. J Am Diet Assoc. 2003. 103:1587 https://www.ncbi.nlm.nih.gov/pubmed/14647078

611 McCrory MA, Howarth NC, Roberts SB, Huang TT. Eating frequency and energy regulation in free-living adults consuming self-selected diets. Journal of Nutrition. 2011. 141:148-153

612 McDonald L, Wardle J, Llewellyn CH, Johnson L, van Jaarsveld CH, Syrad H, Fisher A. Sleep and nighttime energy consumption in early childhood: a population-based cohort study. Pediatr Obes. 2015. 10:454-60 https://www.ncbi.nlm.nih.gov/pubmed/25565402
Daily Eating Occasions Not

Reported, Size of Study Groups

Intervention/Exposure, Daily Eating Occasions Not Reported

Study Design

Daily Eating Occasions Not Reported

Study Design

Intervention/Exposure, Daily Eating Occasions Not Reported

Daily Eating Occasions Not

Reported

Intervention/Exposure, Daily Eating Occasions Not Reported

Study Design

Study Design

Intervention/Exposure, Daily Eating Occasions Not Reported, Comparator 

midwife-led intervention. J Hum Nutr Diet. 2015. 28 Suppl 1:29-37 https://www.ncbi.nlm.nih.gov/pubmed/24809211

614 Mclver VJ, Mattin LR, Evans GH, Yau AMW. Diurnal influences of fasted and non-fasted brisk walking on gastric emptying rate, metabolic responses, and appetite in healthy males. Appetite. 2019. 143:104411 https://www.ncbi.nlm.nih.gov/pubmed/31445052

615 McNeil J, Mamlouk MM, Duval K, Schwartz A, Nardo Junior N, Doucet E. Alterations in metabolic profile occur in normal-weight and obese men during the Ramadan fast despite no changes in anthropometry. J Obes. 2014. 2014:482547 https://www.ncbi.nlm.nih.gov/pubmed/25177497

\section{Megson M, Wing R, Leahey TM. Effects of breakfast eating and eating frequency on body mass index and weight} loss outcomes in adults enrolled in an obesity treatment program. J Behav Med. 2017. 40:595-601 https://www.ncbi.nlm.nih.gov/pubmed/28110468

617 Mejean C, Lampure A, Si Hassen W, Gojard S, Peneau S, Hercberg S, Castetbon K. Influence of food preparation behaviors on 5-year weight change and obesity risk in a French prospective cohort. Int J Behav Nutr Phys Act. 2018. 15:120 https://www.ncbi.nlm.nih.gov/pubmed/30477513

618 Mekary RA. Breakfast Skipping and Type 2 Diabetes: Where Do We Stand?. J Nutr. 2019. 149:1-3 https://www.ncbi.nlm.nih.gov/pubmed/30624661

619 Memari A-H, Kordi R, Panahi N, Nikookar LR, Abdollahi M, Akbarnejad A. Effect of Ramadan Fasting on Body Composition and Physical Performance in Female Athletes. Asian Journal of Sports Medicine. 2011;2(3):161-6. https://www.ncbi.nlm.nih.gov/pubmed/22375235.

620 Mendez-Hernandez P, Dosamantes-Carrasco LD, Siani C, Pierlot R, Martinez-Gomez M, Rivera-Paredez B, et al. Mealtime habits and risk of developing the metabolic syndrome or insulin resistance among Mexican adults. $B r J$ Nutr. 2016:1-10. https://www.ncbi.nlm.nih.gov/pubmed/27842612.

621 Menon JE, Stensel DJ, Tolfrey K, Burns SF. Increased Meal Frequency With Exercise Mitigates Postprandial Triacylglycerol. J Phys Act Health. 2019. 16:589-594 https://www.ncbi.nlm.nih.gov/pubmed/31195871

Intervention/Exposure

Daily Eating Occasions Not

Reported

Eating Frequency Data

Collection

Intervention/Exposure

Study Design, Publication Status

Daily Eating Occasions Not

Reported

Intervention/Exposure

Daily Eating Occasions Not

Reported, Eating Frequency

Data Collection

622 Merl V, Peters A, Oltmanns KM, Kern W, Hubold C, Hallschmid M, Born J, Fehm HL, Schultes B. Preserved circadian rhythm of serum insulin concentration at low plasma glucose during fasting in lean and overweight humans. Metabolism. 2004. 53:1449-53 https://www.ncbi.nlm.nih.gov/pubmed/15536600

Daily Eating Occasions Not Reported 

https://www.ncbi.nlm.nih.gov/pubmed/19631044

\section{4} Mi SJ, Kelly NR, Brychta RJ, Grammer AC, Jaramillo M, Chen KY, Fletcher LA, Bernstein SB, Courville AB, Shank LM, Pomeroy JJ, Brady SM, Broadney MM, Tanofsky-Kraff M, Yanovski JA. Associations of sleep patterns with metabolic syndrome indices, body composition, and energy intake in children and adolescents. Pediatr Obes. 2019. 14:e12507 https://www.ncbi.nlm.nih.gov/pubmed/30702801 early adolescence. J Adolesc Health. 2014. 54:574-81 https://www.ncbi.nlm.nih.gov/pubmed/24360247

626 Michalsen A, Frey UH, Merse S, Siffert W, Dobos GJ. Hunger and mood during extended fasting are dependent on the GNB3 C825T polymorphism. Ann Nutr Metab. 2009. 54:184-8 https://www.ncbi.nlm.nih.gov/pubmed/19420911

Intervention/Exposure

Michalsen A, Hoffmann B, Moebus S, Bäcker M, Langhorst J, Dobos GJ. Incorporation of fasting therapy in an integrative medicine ward: evaluation of outcome, safety, and effects on lifestyle adherence in a large prospective cohort study [corrected] [published erratum appears in J Altern Complement Med 2005 Dec;11(6):1121]. Journal of Alternative \& Complementary Medicine. 2005;11(4):601-7.https://www.ncbi.nlm.nih.gov/pubmed/16131283.

628 Michalsen A, Kuhlmann MK, Ludtke R, Backer M, Langhorst J, Dobos GJ. Prolonged fasting in patients with chronic pain syndromes leads to late mood-enhancement not related to weight loss and fasting-induced leptin depletion. Nutr Neurosci. 2006. 9:195-200 https://www.ncbi.nlm.nih.gov/pubmed/17263085

629 Michalsen A, Schlegel F, Rodenbeck A, Ludtke R, Huether G, Teschler H, Dobos GJ. Effects of short-term modified fasting on sleep patterns and daytime vigilance in non-obese subjects: results of a pilot study. Ann Nutr Metab. 2003. 47:194-200 https://www.ncbi.nlm.nih.gov/pubmed/12748412

630 Michalsen A, Schneider S, Rodenbeck A, Ludtke R, Huether G, Dobos GJ. The short-term effects of fasting on the neuroendocrine system in patients with chronic pain syndromes. Nutr Neurosci. 2003. 6:11-8 https://www.ncbi.nlm.nih.gov/pubmed/12608732 Widhalm K, Kafatos A, Kersting M, Huybrechts I, Moreno LA, Gonzalez-Gross M. Regular breakfast consumption is associated with higher blood vitamin status in adolescents: the HELENA (Healthy Lifestyle in Europe by Nutrition in Adolescence) Study. Public Health Nutr. 2017. 20:1393-1404 https://www.ncbi.nlm.nih.gov/pubmed/28183371

Study Design,

Intervention/Exposure, Daily Eating Occasions Not Reported

Health Status

Daily Eating Occasions Not Reported

\section{Outcome}

Intervention/Exposure, Daily Eating Occasions Not Reported

\section{Study Design}


632 Min C, Noh H, Kang YS, Sim HJ, Baik HW, Song WO, Yoon J, Park YH, Joung H. Skipping breakfast is associated with diet quality and metabolic syndrome risk factors of adults. Nutr Res Pract. 2011. 5:455-63

Study Design, Daily Eating https://www.ncbi.nlm.nih.gov/pubmed/22125684

633 Miranda ER, Varady K, Haus JM. Weight loss via alternate day fasting increases circulating endogenous secretory RAGE and is associated with markers of adipocyte health. FASEB journal. 2017;31(1).

634 Miyamoto S, Murotani K, Yanagawa T, Kato A, Matsunaga S. Relationship of low lean body mass with body weight increase until one year of age and current lifestyles in Japanese young women. $J$ Hum Ergol (Tokyo). 2010. 39:4551 https://www.ncbi.nlm.nih.gov/pubmed/21922790

635 Mojto V, Gvozdjakova A, Kucharska J, Rausova Z, Vancova O, Valuch J. Effects of complete water fasting and regeneration diet on kidney function, oxidative stress and antioxidants. Bratisl Lek Listy. 2018. 119:107-111 https://www.ncbi.nlm.nih.gov/pubmed/29455546

636 Mok A, Haldar S, Lee JC, Leow MK, Henry CJ. Postprandial changes in cardiometabolic disease risk in young Chinese men following isocaloric high or low protein diets, stratified by either high or low meal frequency - a randomized controlled crossover trial. Nutr J. 2016. 15:27 https://www.ncbi.nlm.nih.gov/pubmed/26979583

637 Moller L, Stodkilde-Jorgensen H, Jensen FT, Jorgensen JO. Fasting in healthy subjects is associated with intrahepatic accumulation of lipids as assessed by $1 \mathrm{H}$-magnetic resonance spectroscopy. Clin Sci (Lond). 2008. 114:547-52 https://www.ncbi.nlm.nih.gov/pubmed/17990983

Occasions Not Reported

Study Design, Publication Status

Intervention/Exposure

Study Design,

Intervention/Exposure, Size of

Study Groups

\section{Eating Frequency Data}

Collection

638 Molzof HE, Wirth MD, Burch JB, Shivappa N, Hebert JR, Johnson RL, Gamble KL. The impact of meal timing on Study Design cardiometabolic syndrome indicators in shift workers. Chronobiol Int. 2017. 34:337-348 https://www.ncbi.nlm.nih.gov/pubmed/28107043

639 Moosavi SA, Kabir A, Moghimi A, Chehrei A, Rad MB. Evaluation of the effect of Islamic fasting on lung volumes and capacities in the healthy persons. Saudi Med J. 2007. 28:1666-70 https://www.ncbi.nlm.nih.gov/pubmed/17965786 secretion in healthy volunteers. Br J Nutr. 2012. 108:1286-91 https://www.ncbi.nlm.nih.gov/pubmed/22176632

641 Morimoto A, Ohno Y, Tatsumi Y, Mizuno S, Watanabe S. Effects of healthy dietary pattern and other lifestyle factors on incidence of diabetes in a rural Japanese population. Asia Pac J Clin Nutr. 2012. 21:601-8 https://www.ncbi.nlm.nih.gov/pubmed/23017319 
642 Moro T, Tinsley G, Bianco A, Marcolin G, Pacelli QF, Battaglia G, Palma A, Gentil P, Neri M, Paoli A. Effects of eight weeks of time-restricted feeding (16/8) on basal metabolism, maximal strength, body composition, inflammation, and cardiovascular risk factors in resistance-trained males. J Transl Med. 2016. 14:290 https://www.ncbi.nlm.nih.gov/pubmed/27737674

643 Morris CJ, Purvis TE, Mistretta J, Scheer FA. Effects of the Internal Circadian System and Circadian Misalignment on Glucose Tolerance in Chronic Shift Workers. J Clin Endocrinol Metab. 2016. 101:1066-74 https://www.ncbi.nlm.nih.gov/pubmed/26771705

644 Mostad IL, Langaas M, Grill V. Central obesity is associated with lower intake of whole-grain bread and less frequent Study Design breakfast and lunch: results from the HUNT study, an adult all-population survey. Appl Physiol Nutr Metab. 2014;39(7):819-28. https://www.ncbi.nlm.nih.gov/pubmed/24833275.

645 Mota J, Fidalgo F, Silva R, Ribeiro JC, Santos R, Carvalho J, Santos MP. Relationships between physical activity, obesity and meal frequency in adolescents. Ann Hum Biol. 2008. 35:1-10 https://www.ncbi.nlm.nih.gov/pubmed/18274921

646 Mota MC, Silva CM, Balieiro LCT, Goncalves BF, Fahmy WM, Crispim CA. Association between social jetlag food consumption and meal times in patients with obesity-related chronic diseases. PLoS One. 2019. 14:e0212126 https://www.ncbi.nlm.nih.gov/pubmed/30753224

647 Motohashi Y, Higuchi S, Ahara M, Kaneko Y. Sleep time and working conditions of office workers. Sleep and Biological Rhythms. 2003. 1:131-132

\section{Comparator} Mughal F. Ramadan: what it means for general practice. Br J Gen Pract. 2014. 64:356

\section{Mughal F. Ramadan: what it means for general pra
https://www.ncbi.nlm.nih.gov/pubmed/24982480}

649 Munkholm A, Olsen EM, Rask CU, Clemmensen L, Rimvall MK, Jeppesen P, Micali N, Skovgaard AM. Early

$\begin{array}{ll}\text { Munkholm A, Olsen EM, Rask CU, Clemmensen L, Rimvall MK, Jeppesen P, Micali N, Skovgaard AM. Early } & \text { Intervention/Exposure, Daily } \\ \text { Predictors of Eating Problems in Preadolescence-A Prospective Birth Cohort Study. J Adolesc Health. 2016. 58:533- } & \text { Eating Occasions Not Reported }\end{array}$ 42 https://www.ncbi.nlm.nih.gov/pubmed/27107908

650 Munoz JSG, Canavate R, Hernandez CM, Cara-Salmeron V, Morante JJH. The association among chronotype, timing of food intake and food preferences depends on body mass status. Eur J Clin Nutr. 2017. 71:736-742 https://www.ncbi.nlm.nih.gov/pubmed/27650874

651 Munsters MJ, Saris WH. Effects of meal frequency on metabolic profiles and substrate partitioning in lean healthy males. PLoS One. 2012. 7:e38632 https://www.ncbi.nlm.nih.gov/pubmed/22719910

Study Design,

Intervention/Exposure, Daily Eating Occasions Not Reported

Study Design,

Intervention/Exposure

\section{Study Design}

Intervention/Exposure, Daily Eating Occasions Not Reported

Size of Study Groups

Study Design 
653 Murakami K, Livingstone MBE, Sasaki S. Establishment of a Meal Coding System for the Characterization of MealBased Dietary Patterns in Japan. J Nutr. 2017. 147:2093-2101 https://www.ncbi.nlm.nih.gov/pubmed/28904121

654 Musaiger AO, Al-Roomi K, Bader Z. Social, dietary and lifestyle factors associated with obesity among Bahraini adolescents. Appetite. 2014. 73:197-204 https://www.ncbi.nlm.nih.gov/pubmed/24231426

655 Mzoughi K, Zairi I, Jabeur M, Kraiem S. The effects of fasting on heart rate variability in hypertensive patients. Clin Exp Hypertens. 2018. 40:793-796 https://www.ncbi.nlm.nih.gov/pubmed/29420093

656 Mzoughi K, Zairi I, Jabeur M, Zoghlemi B, Mimouni N, Ben Moussa F, Kamoun S, Fennira S, Kraiem S. P-207: The effects of Ramadan Fasting on heart rate variability in hypertensive patients. Annales de Cardiologie et d'Angeiologie. 2015. 64:S92

657 Nagai M, Uyama O, Kaji H. Dietary habits prone to lifestyle-related disease. Health Education Journal. 2013. 72:172-179

$658 \quad$ Nagata JM, Garber AK, Tabler J, Murray SB, Vittinghoff E, Bibbins-Domingo K. Disordered eating behaviors and cardiometabolic risk among young adults with overweight or obesity. Int J Eat Disord. 2018. 51:931-941 https://www.ncbi.nlm.nih.gov/pubmed/30030944

659 Nagata K, Yoshida M, Ishimoto Y, Hashizume H, Yamada H, Yoshimura N. Skipping breakfast and less exercise are risk factors for bone loss in young Japanese adults: a 3-year follow-up study. J Bone Miner Metab. 2014. 32:420-7 https://www.ncbi.nlm.nih.gov/pubmed/24052206

$660 \quad$ Naharudin MNB, Yusof A. The effect of 10 days of intermittent fasting on Wingate anaerobic power and prolonged high-intensity time-to-exhaustion cycling performance. Eur J Sport Sci. 2018. 18:667-676 https://www.ncbi.nlm.nih.gov/pubmed/29485326

661 Nakajima K, Suwa K. Association of hyperglycemia in a general Japanese population with late-night-dinner eating alone, but not breakfast skipping alone. J Diabetes Metab Disord. 2015. 14:16 https://www.ncbi.nlm.nih.gov/pubmed/25874189

662

Nas A, Hagele F, Kahlhofer J, Keller J, Rising R, Bosy-Westphal A. Impact of breakfast skipping vs. dinner skipping on regulation of energy balance and metabolic risk. Obesity facts. 2017. 10:76

663 Nas A, Mirza N, Hagele F, Kahlhofer J, Keller J, Rising R, Kufer TA, Bosy-Westphal A. Impact of breakfast skipping compared with dinner skipping on regulation of energy balance and metabolic risk. Am J Clin Nutr. 2017. 105:13511361 https://www.ncbi.nlm.nih.gov/pubmed/28490511
Study Design, Outcome

Study Design

Health Status

Daily Eating Occasions Not

Reported, Publication Status

\section{Study Design}

Intervention/Exposure, Daily

Eating Occasions Not Reported

Daily Eating Occasions Not Reported

Size of Study Groups

Study Design

Publication Status

Eating Frequency Data

Collection

Study Design health. J Ky Med Assoc. 2007. 105:223-4 https://www.ncbi.nlm.nih.gov/pubmed/17566468 


\section{Citation}

665

Nematy M, Alinezhad-Namaghi M, Rashed MM, Mozhdehifard M, Sajjadi SS, Akhlaghi S, Sabery M, Mohajeri SA, Shalaey N, Moohebati M, Norouzy A. Effects of Ramadan fasting on cardiovascular risk factors: a prospective observational study. Nutr J. 2012. 11:69 https://www.ncbi.nlm.nih.gov/pubmed/22963582

666 Nemet D, Barzilay-Teeni N, Eliakim A. Treatment of childhood obesity in obese families. J Pediatr Endocrinol Metab. 2008. 21:461-7 https://www.ncbi.nlm.nih.gov/pubmed/18655528

667 Neumann BL, Dunn A, Johnson D, Adams JD, Baum JI. Breakfast Macronutrient Composition Influences Thermic Effect of Feeding and Fat Oxidation in Young Women Who Habitually Skip Breakfast. Nutrients. 2016;8(8). https://www.ncbi.nlm.nih.gov/pubmed/27517958.

668 Neumark-Sztainer D, Wall M, Haines J, Story M, Eisenberg ME. Why does dieting predict weight gain in adolescents? Findings from project EAT-II: a 5-year longitudinal study. J Am Diet Assoc. 2007. 107:448-55 https://www.ncbi.nlm.nih.gov/pubmed/17324664

$669 \quad$ Neumark-Sztainer DR, Wall MM, Haines JI, Story MT, Sherwood NE, van den Berg PA. Shared risk and protective factors for overweight and disordered eating in adolescents. Am J Prev Med. 2007. 33:359-369 https://www.ncbi.nlm.nih.gov/pubmed/17950400

670 Nicklas TA, O'Neil CE, Fulgoni VL. Snacking patterns, diet quality, and cardiovascular risk factors in adults. BMC Public Health. 2014. 14:388 https://www.ncbi.nlm.nih.gov/pubmed/24754905

$671 \quad$ Niemeier BS, Hektner JM. Weight-Related Health Behaviors and Body Mass: Associations between Young Adults and Their Parents, Moderated by Parental Authority. American Journal of Health Education. 2012;43(6):366-77.

672 Niemeier HM, Raynor HA, Lloyd-Richardson EE, Rogers ML, Wing RR. Fast food consumption and breakfast skipping: predictors of weight gain from adolescence to adulthood in a nationally representative sample. J Adolesc Health. 2006. 39:842-9 https://www.ncbi.nlm.nih.gov/pubmed/17116514

673 Nishiura C, Noguchi J, Hashimoto H. Dietary patterns only partially explain the effect of short sleep duration on the incidence of obesity. Sleep. 2010. 33:753-7 https://www.ncbi.nlm.nih.gov/pubmed/20550015

$674 \quad$ Nisselson HS. The Perils of Skipping Breakfast. J N J Dent Assoc. 2015. 86:4 https://www.ncbi.nlm.nih.gov/pubmed/27156267

$675 \quad$ Nola IA, Jelinic JD, Matanic D, Pucarin-Cvetkovic J, Bergman Markovic B, Senta A. Differences in eating and lifestyle habits between first- and sixth-year medical students from Zagreb. Coll Antropol. 2010. 34:1289-94 https://www.ncbi.nlm.nih.gov/pubmed/21874711 Ramadan fasting. East Mediterr Health J. 2005. 11:119-25 https://www.ncbi.nlm.nih.gov/pubmed/16532680

\section{Rationale}

Daily Eating Occasions Not Reported

Study Design,

Intervention/Exposure

Daily Eating Occasions Not

Reported

Intervention/Exposure, Daily

Eating Occasions Not Reported

Intervention/Exposure, Daily

Eating Occasions Not Reported

Study Design

Study Design

Daily Eating Occasions Not Reported

Intervention/Exposure, Daily Eating Occasions Not Reported

Study Design, Daily Eating Occasions Not Reported

Study Design

Daily Eating Occasions Not Reported 
677 Nonino-Borges CB, Martins Borges R, Bavaresco M, Suen VM, Moreira AC, Marchini JS. Influence of meal time on

Comparator salivary circadian cortisol rhythms and weight loss in obese women. Nutrition. 2007. 23:385-91 https://www.ncbi.nlm.nih.gov/pubmed/17483007

678 Nooyens AC, Visscher TL, Schuit AJ, van Rossum CT, Verschuren WM, van Mechelen W, Seidell JC. Effects of retirement on lifestyle in relation to changes in weight and waist circumference in Dutch men: a prospective study. Public Health Nutr. 2005. 8:1266-74 https://www.ncbi.nlm.nih.gov/pubmed/16372922

679 Norouzy A, Hasanzade Daloee M, Khoshnasab AH, Khoshnasab A, Farrokhi J, Nematy M, Safarian M, Nezafati P, Alinezhad-Namaghi M. Trend of blood pressure in hypertensive and normotensive volunteers during Ramadan fasting. Blood Press Monit. 2017. 22:253-257 https://www.ncbi.nlm.nih.gov/pubmed/28657936

680 Norouzy A, Salehi M, Philippou E, Arabi H, Shiva F, Mehrnoosh S, Mohajeri SM, Mohajeri SA, Motaghedi Larijani A, Nematy M. Effect of fasting in Ramadan on body composition and nutritional intake: a prospective study. $J$ Hum Nutr Diet. 2013. 26 Suppl 1:97-104 https://www.ncbi.nlm.nih.gov/pubmed/23679071

681 Norouzy A, Salehi M, Philippou E, Arabi H, Shiva F, Mehrnoosh S, Mohajeri SMR, Reza Mohajeri SA, Motaghed Larijani A, Nematy M. Effect of fasting in Ramadan on body composition and nutritional intake: a prospective study. Journal of Human Nutrition \& Dietetics. 2013. 26:97-104

$682 \quad$ Norrelund H, Nair KS, Nielsen S, Frystyk J, Ivarsen P, Jorgensen JO, Christiansen JS, Moller N. The decisive role of free fatty acids for protein conservation during fasting in humans with and without growth hormone. J Clin Endocrinol Metab. 2003. 88:4371-8 https://www.ncbi.nlm.nih.gov/pubmed/12970312

$683 \quad$ Norrelund H, Nielsen S, Christiansen JS, Jorgensen JO, Moller N. Modulation of basal glucose metabolism and insulin sensitivity by growth hormone and free fatty acids during short-term fasting. Eur J Endocrinol. 2004. 150:77987 https://www.ncbi.nlm.nih.gov/pubmed/15191347

684 Norton L, Parr T, Bardsley RG, Ye H, Tsintzas K. Characterization of GLUT4 and calpain expression in healthy human skeletal muscle during fasting and refeeding. Acta Physiol (Oxf). 2007. 189:233-40 https://www.ncbi.nlm.nih.gov/pubmed/17305703

$685 \quad$ Novaes JF, Franceschini Sdo C, Priore SE. Mother's overweight, parents' constant limitation on the foods and frequent snack as risk factors for obesity among children in Brazil. Arch Latinoam Nutr. 2008. 58:256-64 https://www.ncbi.nlm.nih.gov/pubmed/19137988

Daily Eating Occasions Not Reported

Study Design, Daily Eating Occasions Not Reported

Intervention/Exposure, Daily Eating Occasions Not Reported

Daily Eating Occasions Not Reported

Intervention/Exposure, Size of Study Groups

Intervention/Exposure, Daily Eating Occasions Not Reported

Study Design,

Intervention/Exposure, Daily Eating Occasions Not Reported

Study Design

Daily Eating Occasions Not Reported, Size of Study Groups 

adiposity and abdominal obesity in Malaysian school-aged adolescents. PLoS One. 2013. 8:e59297 https://www.ncbi.nlm.nih.gov/pubmed/23520556

$688 \quad$ Nygaard EB, Orskov C, Almdal T, Vestergaard H, Andersen B. Fasting decreases plasma FGF21 in obese subjects and the expression of FGF21 receptors in adipose tissue in both lean and obese subjects. J Endocrinol. 2018. 239:73-80 https://www.ncbi.nlm.nih.gov/pubmed/30307155

689 O'Brien EC, Geraghty AA, O'Sullivan EJ, Riordan JA, Horan MK, Larkin E, Donnelly J, Mehegan J, Twomey PJ, McAuliffe FM. Five-year follow up of a low glycaemic index dietary randomised controlled trial in pregnancy-no longterm maternal effects of a dietary intervention. Bjog. 2019. 126:514-524 https://www.ncbi.nlm.nih.gov/pubmed/30303614

690 Odegaard AO, Jacobs DR, Steffen LM, Van Horn L, Ludwig DS, Pereira MA. Breakfast frequency and development of metabolic risk. Diabetes Care. 2013. 36:3100-6 https://www.ncbi.nlm.nih.gov/pubmed/23775814

691 Ödlund Olin A, Koochek A, Cederholm T, Ljungqvist O. Minimal effect on energy intake by additional evening meal for frail elderly service flat residents--a pilot study. J Nutr Health Aging. 2008;12(5):295-301. https://www.ncbi.nlm.nih.gov/pubmed/18443710.

692 Ogata H, Hatamoto Y, Goto Y, Tajiri E, Yoshimura E, Kiyono K, Uehara Y, Kawanaka K, Omi N, Tanaka H. Association between breakfast skipping and postprandial hyperglycaemia after lunch in healthy young individuals. $\mathrm{Br}$ J Nutr. 2019. 122:431-440 https://www.ncbi.nlm.nih.gov/pubmed/31486356

693 Ogata H, Kayaba M, Tanaka Y, Yajima K, Iwayama K, Ando A, et al. Effect of skipping breakfast for 6 days on energy metabolism and diurnal rhythm of blood glucose in young healthy Japanese males. Am J Clin Nutr. 2019. https://www.ncbi.nlm.nih.gov/pubmed/31095288.

694 Ohkawara K, Cornier MA, Kohrt WM, Melanson EL. Effects of increased meal frequency on fat oxidation and perceived hunger. Obesity (Silver Spring). 2013. 21:336-43 https://www.ncbi.nlm.nih.gov/pubmed/23404961

695 Okada C, Imano H, Muraki I, Yamada K, Iso H. The Association of Having a Late Dinner or Bedtime Snack and Skipping Breakfast with Overweight in Japanese Women. J Obes. 2019. 2019:2439571 https://www.ncbi.nlm.nih.gov/pubmed/30944735
Okada C, Imano H, Yamagishi K, Cui R, Umesawa M, Maruyama K, Muraki I, Hayama-Terada M, Shimizu Y, Sankai T, Okada T, Kiyama M, Kitamura A, Iso H. Dietary Intake of Energy and Nutrients from Breakfast and Risk of Stroke in The Japanese Population: The Circulatory Risk in Communities Study (CIRCS). J Atheroscler Thromb. 2019. 26:145-153 https://www.ncbi.nlm.nih.gov/pubmed/29899172
Daily Eating Occasions Not Reported, Size of Study Groups

Intervention/Exposure, Daily Eating Occasions Not Reported

Daily Eating Occasions Not Reported

Daily Eating Occasions Not Reported

Daily Eating Occasions Not Reported, Eating Frequency Data Collection

Eating Frequency Data Collection

Eating Frequency Data

Collection

\section{Study Design}

Daily Eating Occasions Not Reported 
697 Okada C, Tabuchi T, Iso H. Association between skipping breakfast in parents and children and childhood overweight/obesity among children: a nationwide 10.5-year prospective study in Japan. Int J Obes (Lond). 2018. 42:1724-1732 https://www.ncbi.nlm.nih.gov/pubmed/29686380

698 Olafsdottir AS, Johannsdottir SS, Arngrimsson SA, Johannsson E. Lifestyle intervention at sea changes body composition, metabolic profile and fitness. Public Health. 2012;126(10):888-90.

Daily Eating Occasions Not Reported https://www.ncbi.nlm.nih.gov/pubmed/22884861.

699 Olejniczak D, Bugajec D, Staniszewska A, Panczyk M, Kielan A, Czerw A, Manczuk M, Juszczyk G, Skonieczna J, Brytek-Matera A. Risk assessment of night-eating syndrome occurrence in women in Poland, considering the obesity factor in particular. Neuropsychiatr Dis Treat. 2018. 14:1521-1526 https://www.ncbi.nlm.nih.gov/pubmed/29942130

700 Olson CM, Strawderman MS, Hinton PS, Pearson TA. Gestational weight gain and postpartum behaviors associated with weight change from early pregnancy to 1 y postpartum. International journal of obesity. 2003;27(1):117-27. https://www.ncbi.nlm.nih.gov/pubmed/12532163.

701 Olson J, Aldrich H, Callahan TJ, Matthews EE, Gance-Cleveland B. Characterization of Childhood Obesity and Behavioral Factors. J Pediatr Health Care. 2016. 30:444-52 https://www.ncbi.nlm.nih.gov/pubmed/26614274

Intervention/Exposure, Daily Eating Occasions Not Reported

Study Design, Daily Eating Occasions Not Reported

702 Ong KK. Summary of Factors Influencing Healthy Growth. Nestle Nutr Inst Workshop Ser. 2017. 87:197-199 https://www.ncbi.nlm.nih.gov/pubmed/28315899

703 O'Reardon JP, Ringel BL, Dinges DF, Allison KC, Rogers NL, Martino NS, Stunkard AJ. Circadian eating and sleeping patterns in the night eating syndrome. Obes Res. 2004. 12:1789-96 https://www.ncbi.nlm.nih.gov/pubmed/15601974

Intervention/Exposure, Daily Eating Occasions Not Reported

Daily Eating Occasions Not Reported, Eating Frequency Data Collection

Publication Status

$704 \quad$ Orra AA, Pires MM, Ferreira SR. Distinct breakfast patterns on satiety perception in individuals with weight excess. Arch Endocrinol Metab. 2016. 60:333-40 https://www.ncbi.nlm.nih.gov/pubmed/26910624

Intervention/Exposure, Daily Eating Occasions Not Reported, Outcome

Intervention/Exposure,

Comparator

705 Oshida $\mathrm{H}$, Kutsuma A, Nakajima K. Associations of eating a late-evening meal before bedtime with low serum amylase and unhealthy conditions. J Diabetes Metab Disord. 2013. 12:53 https://www.ncbi.nlm.nih.gov/pubmed/24354901

Daily Eating Occasions Not Reported 

prevention of future chronic diseases. Tohoku J Exp Med. 2009. 217:67-72 https://www.ncbi.nlm.nih.gov/pubmed/19155610

708 Ozturk E, Balat O, Ugur MG, Yazicioglu C, Pence S, Erel O, Kul S. Effect of Ramadan fasting on maternal oxidative stress during the second trimester: a preliminary study. J Obstet Gynaecol Res. 2011. 37:729-33 https://www.ncbi.nlm.nih.gov/pubmed/21736666

709

Palla L, Almoosawi S. Diurnal Patterns of Energy Intake Derived via Principal Component Analysis and Their Relationship with Adiposity Measures in Adolescents: Results from the National Diet and Nutrition Survey RP (2008(-)2012). Nutrients. 2019;11(2). https://www.ncbi.nlm.nih.gov/pubmed/30781551.

710 Pallayova M, Zaghloul HB, Arora T, Choudhury SM, Omar OM, Chagoury OL, Taheri S. Investigating physiological glucose excursions before, during, and after Ramadan in adults without diabetes mellitus. Physiol Behav. 2017. 179:110-115 https://www.ncbi.nlm.nih.gov/pubmed/28577886

711 Palmer MA, Capra S, Baines SK. Association between eating frequency, weight, and health. Nutrition reviews. 2009;67(7):379-90.https://www.ncbi.nlm.nih.gov/pubmed/19566598.

712 Panagiotopoulos C, Riddell MC, Sellers EAC. Type 2 Diabetes in Children and Adolescents. Canadian Journal of Diabetes. 2013. 37:S163-S167.

713 Panizza CE, Lim U, Yonemori KM, Cassel KD, Wilkens LR, Harvie MN, et al. Effects of Intermittent Energy Restriction Combined with a Mediterranean Diet on Reducing Visceral Adiposity: A Randomized Active Comparator Pilot Study. Nutrients. 2019;11(6). https://www.ncbi.nlm.nih.gov/pubmed/31226790.

714 Papakonstantinou E, Kechribari I, Mitrou P, Trakakis E, Vassiliadi D, Georgousopoulou E, Zampelas A, Kontogianni MD, Dimitriadis G. Effect of meal frequency on glucose and insulin levels in women with polycystic ovary syndrome: a randomised trial. Eur J Clin Nutr. 2016. 70:588-94 https://www.ncbi.nlm.nih.gov/pubmed/26862008

715 Papakonstantinou E, Kechribari I, Mitrou P, Trakakis E, Vassiliadi D, Georgousopoulou E, Zampelas A, Kontogianni MD, Dimitriadis G. Effect of meal frequency on glucose levels in women with polycystic ovary syndrome: a randomized trial. Obesity facts. 2015. 8:73-74

716 Papakonstantinou E, Kontogianni MD, Mitrou P, Magriplis E, Vassiliadi D, Nomikos T, Lambadiari V, Georgousopoulou E, Dimitriadis G. Effects of 6 vs 3 eucaloric meal patterns on glycaemic control and satiety in people with impaired glucose tolerance or overt type 2 diabetes: A randomized trial. Diabetes Metab. 2018. 44:226234 https://www.ncbi.nlm.nih.gov/pubmed/29680359
Daily Eating Occasions Not Reported

Study Design

Daily Eating Occasions Not Reported

Study Design

Study Design

Daily Eating Occasions Not

Reported

Health Status

Health Status

Eating Frequency Data Collection 
717 Papoutsou S, Briassoulis G, Hadjigeorgiou C, Savva SC, Solea T, Hebestreit A, Pala V, Sieri S, Kourides Y, Kafatos $\mathrm{A}$, Tornaritis $\mathrm{M}$. The combination of daily breakfast consumption and optimal breakfast choices in childhood is an Study Design, Daily Eating important public health message. Int J Food Sci Nutr. 2014. 65:273-9 https://www.ncbi.nlm.nih.gov/pubmed/24512299

718 Park EH, Oh MS, Kim S, Lee J, Kang KS. The Analysis of Factors Causing the High Prevalence of Child Obesity in Jeju Island. Pediatr Gastroenterol Hepatol Nutr. 2018. 21:127-133 https://www.ncbi.nlm.nih.gov/pubmed/29713610

719 Park HJ, Lee J, Kim JM, Lee HA, Kim SH, Kim Y. A study of snack consumption, night-eating habits, and nutrient

intake in gestational diabetes mellitus. Clin Nutr Res. 2013. 2:42-51 https://www.ncbi.n/m.nih.gov/pubmed/23431085

Occasions Not Reported

720 Parkes KR. Demographic and lifestyle predictors of body mass index among offshore oil industry workers: crosssectional and longitudinal findings. Occup Med (Lond). 2003. 53:213-21 https://www.ncbi.nlm.nih.gov/pubmed/12724556

721 Parr EB, Devlin BL, Brennan L, Hawley JA. Controlled time-restricted eating alters ratings of hunger, but does not change fatigue and mood in men with overweight/obesity. Obesity facts. 2019. 12:260

Study Design

Study Design,

Intervention/Exposure, Daily Eating Occasions Not Reported

Intervention/Exposure

Parr EB, Devlin BL, Brennan L, Hawley JA. Effects of time-restricted feeding on mood, hunger and fatigue. Obesity

Parr EB, Devlin BL, Brennan L, Hawley JA
research \& clinical practice. 2019. 13:245

723 Parretti HM, Aveyard P, Blannin A, Clifford SJ, Coleman SJ, Roalfe A, Daley AJ. Efficacy of water preloading before main meals as a strategy for weight loss in primary care patients with obesity: RCT. Obesity (Silver Spring). 2015. 23:1785-91 https://www.ncbi.nlm.nih.gov/pubmed/26237305

724 Partridge C. Unhealthy lifestyle in college students could lead to diabetes risk. Nephrol News Issues. 2007. 21:22, 25 https://www.ncbi.nlm.nih.gov/pubmed/17269261

Pasqua IC, Moreno CR. The nutritional status and eating habits of shift workers: a chronobiological approach. Chronobiol Int. 2004. 21:949-60 https://www.ncbi.nlm.nih.gov/pubmed/15646241 https://www.ncbi.nlm.nih.gov/pubmed/25857868

Daily Eating Occasions Not Reported

\section{Study Design,}

Intervention/Exposure

Publication Status

Intervention/Exposure, Daily Eating Occasions Not Reported

Study Design

Intervention/Exposure 

hunger, and eating patterns. Int J Obes Relat Metab Disord. 2003. 27:970-8 https://www.ncbi.nlm.nih.gov/pubmed/12861239

729 Pearcey SM, de Castro JM. Food intake and meal patterns of weight-stable Nutr. 2002. 76:107-12 https://www.ncbi.nlm.nih.gov/pubmed/12081823

\section{0}

\section{Peder}

Pedersen E, Jennifer BKeoghJ, Kristina Petersen K, Peter M, Clifton P. Effects of intermittent compared to continuous energy restriction on weight loss and diet quality after one year. Obesity reviews. 2014. 15:142

731 Pedersen MH, Svart MV, Lebeck J, Bidlingmaier M, Stodkilde-Jorgensen H, Pedersen SB, Moller N, Jessen N, Jorgensen JOL. Substrate Metabolism and Insulin Sensitivity During Fasting in Obese Human Subjects: Impact of GH Blockade. J Clin Endocrinol Metab. 2017. 102:1340-1349 https://www.ncbi.nlm.nih.gov/pubmed/28324055

Pelkman CL, Heinbach RA, Rolls BJ. Reproductive hormones and eating behavior in young women. Appetite. 2000. 34:217-8 https://www.ncbi.nlm.nih.gov/pubmed/10744913

Pentikainen S, Tanner H, Karhunen L, Kolehmainen M, Poutanen K, Pennanen K. Mobile Phone App for SelfMonitoring of Eating Rhythm: Field Experiment. JMIR Mhealth Uhealth. 2019. 7:e11490 https://www.ncbi.nlm.nih.gov/pubmed/30916657

734 Peos JJ, Helms ER, Fournier PA, Sainsbury A. Continuous versus intermittent moderate energy restriction for increased fat mass loss and fat free mass retention in adult athletes: protocol for a randomised controlled trial-the ICECAP trial (Intermittent versus Continuous Energy restriction Compared in an Athlete Population). BMJ Open Sport Exerc Med. 2018. 4:e000423 https://www.ncbi.nlm.nih.gov/pubmed/30364484

$735 \quad$ Pereira JL, Felix PV, Mattei J, Fisberg RM. Differences over 12 Years in Food Portion Size and Association with Excess Body Weight in the City of Sao Paulo, Brazil. Nutrients. 2018;10(6). https://www.ncbi.nlm.nih.gov/pubmed/29848971.

736 Pereira MA, Erickson E, McKee P, Schrankler K, Raatz SK, Lytle LA, et al. Breakfast frequency and quality may affect glycemia and appetite in adults and children. Journal of nutrition. 2011;141(1):163-8. https://www.ncbi.nlm.nih.gov/pubmed/21123469.

737 Perk G, Ghanem J, Aamar S, Ben-Ishay D, Bursztyn M. The effect of the fast of Ramadan on ambulatory blood pressure in treated hypertensives. J Hum Hypertens. 2001. 15:723-5 https://www.ncbi.nlm.nih.gov/pubmed/11607803

Study Design,

Intervention/Exposure, Daily Eating Occasions Not Reported

Study Design

Intervention/Exposure, Daily Eating Occasions Not Reported

Study Design

Outcome, Size of Study Groups

Study Design

Intervention/Exposure, Daily Eating Occasions Not Reported

Outcome, Size of Study Groups 


\section{Perrigue MM, Kantor ED, Hastert TA, Patterson R, Potter JD, Neuhouser ML, White E. Eating frequency and risk of Outcome} colorectal cancer. Cancer Causes Control. 2013. 24:2107-15 https://www.ncbi.nlm.nih.gov/pubmed/24057417

$741 \quad$ Peterson CM. Intermittent Fasting Induces Weight Loss, but the Effects on Cardiometabolic Health are Modulated by Energy Balance. Obesity (Silver Spring). 2019. 27:11 https://www.ncbi.nlm.nih.gov/pubmed/30569643

742 Petherick ES, Tuffnell D, Wright J. Experiences and outcomes of maternal Ramadan fasting during pregnancy: results from a sub-cohort of the Born in Bradford birth cohort study. BMC Pregnancy Childbirth. 2014. 14:335 https://www.ncbi.nlm.nih.gov/pubmed/25261183

743 Petrella E, Facchinetti F, Bertarini V, Pignatti L, Neri I, Battistini NC. Occurrence of pregnancy complications in women with BMI >25 submitted to a healthy lifestyle and eating habits program. American journal of obstetrics and gynecology. 2013. 208:S33-S34

744 Petrella E, Malavolti M, Bertarini V, Pignatti L, Neri I, Battistini NC, Facchinetti F. Gestational weight gain in overweight and obese women enrolled in a healthy lifestyle and eating habits program. J Matern Fetal Neonatal Med. 2014. 27:1348-52 https://www.ncbi.nlm.nih.gov/pubmed/24175912

745 Phelan S, Phipps MG, Abrams B, Darroch F, Schaffner A, Wing RR. Randomized trial of a behavioral intervention to prevent excessive gestational weight gain: the fit for delivery study. Obstetrical \& gynecological survey. 2011. $66: 471-472$

746 Phelan S, Wing RR, Brannen A, McHugh A, Hagobian TA, Schaffner A, Jelalian E, Hart CN, Scholl TO, MunozChristian K, Yin E, Phipps MG, Keadle S, Abrams B. Randomized controlled clinical trial of behavioral lifestyle intervention with partial meal replacement to reduce excessive gestational weight gain. Am J Clin Nutr. 2018. 107:183-194 https://www.ncbi.nlm.nih.gov/pubmed/29529157

747 Phelan S, Wyatt HR, Hill JO, Wing RR. Are the eating and exercise habits of successful weight losers changing?. Obesity (Silver Spring). 2006. 14:710-6 https://www.ncbi.nlm.nih.gov/pubmed/16741274

748 Pimenta AM, Bes-Rastrollo M, Gea A, Sayon-Orea C, Zazpe I, Lopez-Iracheta R, Martinez-Gonzalez MA. Snacking between main meals is associated with a higher risk of metabolic syndrome in a Mediterranean cohort: the SUN Project (Seguimiento Universidad de Navarra). Public Health Nutr. 2016. 19:658-66 https://www.ncbi.nlm.nih.gov/pubmed/25958949 


\section{Citation}

749 Pincelli Al, Brunani A, Caumo A, Scacchi M, Pasqualinotto L, Tibaldi A, Dubini A, Bonadonna S, Cavagnini F. Hyperinsulinemia in the physiologic range is not superior to short-term fasting in suppressing insulin secretion in obese men. Metabolism. 2001. 50:107-11 https://www.ncbi.nlm.nih.gov/pubmed/11172483

750 Pinto A, Bordoli C, Buckner L, Kaplan P, Arenal I, Jefcock E, Kim C, Johnston K, Hall W. A randomised controlled trial assessing the impact of intermittent energy restriction (IER) on weight loss and insulin sensitivity in healthy men and women with central obesity. "the Met-IER study". Obesity facts. 2017. 10:157

751 Pinto AM, Bordoli C, Buckner LP, Kim C, Kaplan PC, Del Arenal IM, et al. Intermittent energy restriction is comparable to continuous energy restriction for cardiometabolic health in adults with central obesity: A randomized controlled trial; the Met-IER study. Clin Nutr. 2019. https://www.ncbi.nlm.nih.gov/pubmed/31409509.

752 Pizinger T, Kovtun K, RoyChoudhury A, Laferrere B, Shechter A, St-Onge MP. Pilot study of sleep and meal timing effects, independent of sleep duration and food intake, on insulin sensitivity in healthy individuals. Sleep Health. 2018. 4:33-39 https://www.ncbi.nlm.nih.gov/pubmed/29332677

$753 \quad$ Plata-Salaman CR. Ingestive behavior and obesity. Nutrition. 2000. 16:797-9 https://www.ncbi.nlm.nih.gov/pubmed/11054583

754 Ple. With a substantial breakfast to control hyperglycemia. MMW fortschritte der medizin. $2015 ; 157$ Suppl 1:9. https://www.ncbi.nlm.nih.gov/pubmed/26012964.

755 Poggiogalle E, Lubrano C, Gnessi L, Marocco C, Di Lazzaro L, Polidoro G, Luisi F, Merola G, Mariani S, Migliaccio S, Lenzi A, Donini LM. Reduced sleep duration affects body composition, dietary intake and quality of life in obese subjects. Eat Weight Disord. 2016. 21:501-505 https://www.ncbi.nlm.nih.gov/pubmed/26911383

756 Ponzo V, Gentile L, Gambino R, Rosato R, Cioffi I, Pellegrini N, Benso A, Broglio F, Cassader M, Bo S. Incidence of diabetes mellitus, cardiovascular outcomes and mortality after a 12-month lifestyle intervention: a 9-year follow-up. Diabetes \& metabolism. 2018. 44:449-451

757 Poston WS, Haddock CK, Pinkston MM, Pace P, Karakoc ND, Reeves RS, Foreyt JP. Weight loss with meal replacement and meal replacement plus snacks: a randomized trial. Int J Obes (Lond). 2005. 29:1107-14 https://www.ncbi.nlm.nih.gov/pubmed/15925955

758

Pot GK, Hardy R, Stephen AM. Irregular consumption of energy intake in meals is associated with a higher cardiometabolic risk in adults of a British birth cohort. Int J Obes (Lond). 2014. 38:1518-24 https://www.ncbi.nlm.nih.gov/pubmed/24675713

Pot GK, Hardy R, Stephen AM. Irregularity of energy intake at meals: prospective associations with the metabolic syndrome in adults of the 1946 British birth cohort. Br J Nutr. 2016. 115:315-23 https://www.ncbi.nlm.nih.gov/pubmed/26548599

\section{Rationale}

Intervention/Exposure, Daily

Eating Occasions Not Reported

Size of Study Groups

Publication Status

Intervention/Exposure

Comparator, Size of Study

Groups

Study Design

Language

Intervention/Exposure

Study Design, Publication Status

Intervention/Exposure

Intervention/Exposure

Intervention/Exposure

Comparator 
761 Purslow LR, Sandhu MS, Forouhi N, Young EH, Luben RN, Welch AA, Khaw K, Bingham SA, Wareham NJ. Energy intake at breakfast and weight change: prospective study of 6,764 middle-aged men and women. American journal of epidemiology. 2008;167(2):188-92. https://www.ncbi.nlm.nih.gov/pubmed/18079134.

762 Quante M, Mariani S, Weng J, Marinac CR, Kaplan ER, Rueschman M, Mitchell JA, James P, Hipp JA, Cespedes Feliciano EM, Wang R, Redline S. Zeitgebers and their association with rest-activity patterns. Chronobiol Int. 2019. 36:203-213 https://www.ncbi.nlm.nih.gov/pubmed/30365354

763 Quick V, Wall M, Larson N, Haines J, Neumark-Sztainer D. Personal, behavioral and socio-environmental predictors of overweight incidence in young adults: 10-yr longitudinal findings. Int J Behav Nutr Phys Act. 2013. 10:37 https://www.ncbi.nlm.nih.gov/pubmed/23531253

764 Rabiee S, Afghari N, Rastmanesh R. Short-term and mid-term effects of fasting and downset meal pattern on lipid profile in Iranian fasted women. J Relig Health. 2014. 53:654-7 https://www.ncbi.nlm.nih.gov/pubmed/23111814

765 Racinais S, Periard JD, Li CK, Grantham J. Activity patterns, body composition and muscle function during Ramadan in a Middle-East Muslim country. Int J Sports Med. 2012. 33:641-6 https://www.ncbi.nlm.nih.gov/pubmed/22510804

766 Radhakishun N, Blokhuis C, van Vliet M, von Rosenstiel I, Weijer O, Heymans M, Beijnen J, Brandjes D, Diamant M. Intermittent fasting during Ramadan causes a transient increase in total, LDL, and HDL cholesterols and hs-CRP in ethnic obese adolescents. Eur J Pediatr. 2014. 173:1103-6 https://www.ncbi.nlm.nih.gov/pubmed/24522324

767 Raffoul A, Leatherdale ST, Kirkpatrick SI. Dieting predicts engagement in multiple risky behaviours among adolescent Canadian girls: a longitudinal analysis. Can J Public Health. 2018. 109:61-69 https://www.ncbi.nlm.nih.gov/pubmed/29981072

$768 \quad$ Rahbar AR, Safavi E, Rooholamini M, Jaafari F, Darvishi S, Rahbar A. Effects of Intermittent Fasting during Ramadan on Insulin-like Growth Factor-1, Interleukin 2, and Lipid Profile in Healthy Muslims. Int J Prev Med. 2019. 10:7 https://www.ncbi.nlm.nih.gov/pubmed/30774841

Rakicioglu N, Samur G, Topcu A, Topcu AA. The effect of Ramadan on maternal nutrition and composition of breas milk. Pediatr Int. 2006. 48:278-83 https://www.ncbi.nlm.nih.gov/pubmed/16732795
Intervention/Exposure, Daily Eating Occasions Not Reported

Study Design, Daily Eating

Occasions Not Reported

Daily Eating Occasions Not

Reported, Comparator

Daily Eating Occasions Not Reported

Daily Eating Occasions Not Reported

Daily Eating Occasions Not Reported

Intervention/Exposure, Daily Eating Occasions Not Reported

Daily Eating Occasions Not

Reported, Eating Frequency Data Collection

\section{Outcome}


771 Ramadan JM, Barac-Nieto M. Cardio-respiratory responses to moderately heavy aerobic exercise during the Ramadan fasts. Saudi Med J. 2000. 21:238-44 https://www.ncbi.nlm.nih.gov/pubmed/11533791

772 Ramiro-Gonzalez MD, Sanz-Barbero B, Royo-Bordonada MA. Childhood Excess Weight in Spain From 2006 to 2012. Determinants and Parental Misperception. Rev Esp Cardiol (Engl Ed). 2017. 70:656-663 https://www.ncbi.nlm.nih.gov/pubmed/28330819

773 Ransom T, Goldenberg R, Mikalachki A, Prebtani APH, Punthakee Z. Reducing the Risk of Developing Diabetes. Canadian journal of diabetes. 2013;37(Suppl.1):S16-S9. https://www.ncbi.nlm.nih.gov/pubmed/24070941.

774 Rashidi MR, Mahboob S, Sattarivand R. Effects of nibbling and gorging on lipid profiles, blood glucose and insulin levels in healthy subjects. Saudi Med J. 2003. 24:945-8 https://www.ncbi.nlm.nih.gov/pubmed/12973474

$775 \quad$ Rasmussen F, Kark M, Tholin S, Karnehed N, Tynelius P. The Swedish Young Male Twins Study: a resource for longitudinal research on risk factors for obesity and cardiovascular diseases. Twin Res Hum Genet. 2006. 9:883-9 https://www.ncbi.nlm.nih.gov/pubmed/17254425

776 Ravussin E, Beyl RA, Poggiogalle E, Hsia DS, Peterson CM. Early Time-Restricted Feeding Reduces Appetite and Increases Fat Oxidation But Does Not Affect Energy Expenditure in Humans. Obesity (Silver Spring). 2019. 27:1244-1254 https://www.ncbi.nlm.nih.gov/pubmed/31339000

777 Reeves S, Huber JW, Halsey LG, Horabady-Farahani Y, ljadi M, Smith T. Experimental manipulation of breakfast in normal and overweight/obese participants is associated with changes to nutrient and energy intake consumption patterns. Physiol Behav. 2014. 133:130-5 https://www.ncbi.nlm.nih.gov/pubmed/24866910

778 Reeves S, Huber JW, Halsey LG, Villegas-Montes M, Elgumati J, Smith T. A cross-over experiment to investigate possible mechanisms for lower BMls in people who habitually eat breakfast. Eur J Clin Nutr. 2015. 69:632-7 https://www.ncbi.nlm.nih.gov/pubmed/25563734

779 Reiches MW, Moore SE, Prentice AM, Ellison PT. Endocrine responses, weight change, and energy sparing mechanisms during Ramadan among Gambian adolescent women. Am J Hum Biol. 2014. 26:395-400 https://www.ncbi.nlm.nih.gov/pubmed/24590590 Snault KM, Carlsen EM, Norgaard K, Nilas L, Pryds O, Secher NJ, Olsen SF, Halldorsson TI. Intake of Sweet
Intervention/Exposure, Daily Eating Occasions Not Reported

Study Design

Study Design

Country

Study Design

Intervention/Exposure

Outcome

Intervention/Exposure, Daily Eating Occasions Not Reported

Country

Study Design, Comparator

Intervention/Exposure Randomised Controlled Trial. PLoS One. 2015. 10:e0133041 https://www.ncbi.nlm.nih.gov/pubmed/26192183 

status. ScientificWorldJournal. 2013. 2013:308041 https://www.ncbi.nlm.nih.gov/pubmed/24311975

783 Rong S, Snetselaar LG, Xu G, Sun Y, Liu B, Wallace RB, Bao W. Association of Skipping Breakfast With Cardiovascular and All-Cause Mortality. J Am Coll Cardiol. 2019. 73:2025-2032

Eating Occasions Not Reported https://www.ncbi.nlm.nih.gov/pubmed/31023424

784 Rosbach S, Diederichs T, Bolzenius K, Buyken A, Alexy U. Age and time trends of circadian eating pattern in children and adolescents. Annals of nutrition and metabolism. Conference: $12^{\text {th }}$ european nutrition conference. 2015. 67:156

785 Rosi A, Martini D, Scazzina F, Dall'Aglio E, Leonardi R, Monti L, Fasano F, Di Dio C, Riggio L, Brighenti F. Nature and Cognitive Perception of 4 Different Breakfast Meals Influence Satiety-Related Sensations and Postprandial Metabolic Responses but Have Little Effect on Food Choices and Intake Later in the Day in a Randomized Crossover Trial in Healthy Men. J Nutr. 2018. 148:1536-1546 https://www.ncbi.nlm.nih.gov/pubmed/30204905

\section{6 Rosi A, Scazzina F, Brighenti F. Impact of different breakfast meals on food choices and eating behaviors. Annals of \\ Publication Status} nutrition and metabolism. Conference: $12^{\text {th }}$ european nutrition conference. 2015. 67:133 https://doi.org/10.1159/000440895

787 Rothacker DQ, Staniszewski BA, Ellis PK. Liquid meal replacement vs traditional food: a potential model for women who cannot maintain eating habit change. J Am Diet Assoc. 2001. 101:345-7 https://www.ncbi.nlm.nih.gov/pubmed/11269616

788 Ruge T, Svensson M, Eriksson JW, Olivecrona G. Tissue-specific regulation of lipoprotein lipase in humans: effects of fasting. Eur J Clin Invest. 2005. 35:194-200 https://www.ncbi.nlm.nih.gov/pubmed/15733074

Ruiz-Lozano T, Vidal J, de Hollanda A, Canteras M, Garaulet M, Izquierdo-Pulido M. Evening chronotype associates
with obesity in severely obese subjects: interaction with CLOCK 3111T/C. Int J Obes (Lond). 2016. 40:1550-1557 https://www.ncbi.nlm.nih.gov/pubmed/27339606

790 Saadatnia M, Zare M, Fatehi F, Ahmadi A. The effect of fasting on cerebral venous and dural sinus thrombosis. Neurol Res. 2009. 31:794-8 https://www.ncbi.nlm.nih.gov/pubmed/19723447

Intervention/Exposure,

Comparator

Eating Frequency Data Collection

Publication Status

Intervention/Exposure, Daily Eating Occasions Not Reported, Comparator

Intervention/Exposure

Health Status

Study Design, Daily Eating Occasions Not Reported

Intervention/Exposure, Daily Eating Occasions Not Reported 

2011. 4:409-16 https://www.ncbi.nlm.nih.gov/pubmed/22253539

trimester of pregnancy. BMC Pregnancy Childbirth. 2019. 19:128 https://www.ncbi.nlm.nih.gov/pubmed/30987614

Reported

794 Sakamaki R, Amamoto R, Mochida Y, Shinfuku N, Toyama K. A comparative study of food habits and body shape perception of university students in Japan and Korea. Nutr J. 2005. 4:31

Study Design https://www.ncbi.nlm.nih.gov/pubmed/16255785

795 Sakar MN, Gultekin H, Demir B, Bakir VL, Balsak D, Vuruskan E, Acar H, Yucel O, Yayla M. Ramadan fasting and pregnancy: implications for fetal development in summer season. $J$ Perinat Med. 2015. 43:319-23 https://www.ncbi.nlm.nih.gov/pubmed/24810552

\begin{tabular}{|c|c|c|}
\hline 796 & $\begin{array}{l}\text { Sakurai M, Yoshita K, Nakamura K, Miura K, Takamura T, Nagasawa SY, Morikawa Y, Kido T, Naruse Y, Nogawa } \\
\text { K, Suwazono Y, Sasaki S, Ishizaki M, Nakagawa H. Skipping breakfast and 5-year changes in body mass index and } \\
\text { waist circumference in Japanese men and women. Obes Sci Pract. 2017. 3:162-170 } \\
\text { https://www.ncbi.nlm.nih.gov/pubmed/28702211 }\end{array}$ & $\begin{array}{l}\text { Daily Eating Occasions Not } \\
\text { Reported }\end{array}$ \\
\hline 797 & $\begin{array}{l}\text { Saleh SA, El-Kemery TA, Farrag KA, Badawy MR, Sarkis NN, Soliman FS, Mangoud H. Ramadan fasting: relation } \\
\text { to atherogenic risk among obese Muslims. J Egypt Public Health Assoc. 2004. 79:461-83 } \\
\text { https://www.ncbi.nlm.nih.gov/pubmed/17265611 }\end{array}$ & $\begin{array}{l}\text { Daily Eating Occasions Not } \\
\text { Reported }\end{array}$ \\
\hline 798 & $\begin{array}{l}\text { Salehi M, Neghab M. Effects of fasting and a medium calorie balanced diet during the holy month Ramadan on } \\
\text { weight, BMI and some blood parameters of overweight males. Pak J Biol Sci. 2007. 10:968-71 } \\
\text { https://www.ncbi.nlm.nih.gov/pubmed/19069900 }\end{array}$ & Study Design, Comparator \\
\hline 799 & $\begin{array}{l}\text { Samad F, Qazi F, Pervaiz MB, Kella DK, Mansoor M, Osmani BZ, Mir F, Kadir MM. Effects of ramadan fasting on } \\
\text { blood pressure in normotensive males. J Ayub Med Coll Abbottabad. 2015. 27:338-42 } \\
\text { https://www.ncbi.nlm.nih.gov/pubmed/264111111 }\end{array}$ & $\begin{array}{l}\text { Intervention/Exposure, Daily } \\
\text { Eating Occasions Not Reported }\end{array}$ \\
\hline 800 & $\begin{array}{l}\text { Sandercock GR, Voss C, Dye L. Associations between habitual school-day breakfast consumption, body mass } \\
\text { index, physical activity and cardiorespiratory fitness in English schoolchildren. Eur J Clin Nutr. 2010. 64:1086-92 } \\
\text { https://www.ncbi.nlm.nih.gov/pubmed/20683459 }\end{array}$ & Study Design \\
\hline 801 & $\begin{array}{l}\text { Sandhu SK, Tang TS. When's dinner? Does timing of dinner affect the cardiometabolic risk profiles of South-Asian } \\
\text { Canadians at risk for diabetes. Diabet Med. 2017. 34:539-542 https://www.ncbi.nlm.nih.gov/pubmed/26802477 }\end{array}$ & Study Design \\
\hline
\end{tabular}




\section{Citation}

802 Sarraf-Zadegan N, Atashi M, Naderi GA, Baghai AM, Asgary S, Fatehifar MR, Samarian H, Zarei M. The effect of fasting in Ramadan on the values and interrelations between biochemical, coagulation and hematological factors. Ann Saudi Med. 2000. 20:377-81 https://www.ncbi.nlm.nih.gov/pubmed/17264626

803 Sarri KO, Linardakis MK, Bervanaki FN, Tzanakis NE, Kafatos AG. Greek Orthodox fasting rituals: a hidden characteristic of the Mediterranean diet of Crete. Br J Nutr. 2004. 92:277-84 https://www.ncbi.nlm.nih.gov/pubmed/15333159

804 Sarri KO, Tzanakis NE, Linardakis MK, Mamalakis GD, Kafatos AG. Effects of Greek Orthodox Christian Church fasting on serum lipids and obesity. BMC Public Health. 2003. 3:16 https://www.ncbi.nlm.nih.gov/pubmed/12753698

805 Sasaki S, Nakae S, Ebine N, Aoi W, Higashi A, Ishii K. The effect of the timing of meal intake on energy metabolism during moderate exercise. J Nutr Sci Vitaminol (Tokyo). 2014. 60:28-34 https://www.ncbi.nlm.nih.gov/pubmed/24759257

806 Sassi M, Chakroun T, Chouchene S, Hellara I, Boubaker H, Grissa MH, Khochtali I, Hassine M, Addad F, Elalamy I Nouira S. Does Lipid Profile Affect Thrombin Generation During Ramadan Fasting in Patients With Cardiovascular Risks?. Clin Appl Thromb Hemost. 2017. 23:980-986 https://www.ncbi.nlm.nih.gov/pubmed/27613563

807 Sata M, Yamagishi K, Sairenchi T, Ikeda A, Irie F, Watanabe H, Iso H, Ota H. Impact of Caregiver Type for 3-YearOld Children on Subsequent Between-Meal Eating Habits and Being Overweight From Childhood to Adulthood: A 20-Year Follow-up of the Ibaraki Children's Cohort (IBACHIL) Study. J Epidemiol. 2015. 25:600-7 https://www.ncbi.nlm.nih.gov/pubmed/26310570

808 Sato M, Nakamura K, Ogata H, Miyashita A, Nagasaka S, Omi N, Yamaguchi S, Hibi M, Umeda T, Nakaji S, Tokuyama K. Acute effect of late evening meal on diurnal variation of blood glucose and energy metabolism. Obes Res Clin Pract. 2011. 5:e169-266 https://www.ncbi.nlm.nih.gov/pubmed/24331104

809 Savitri AI, Yadegari N, Bakker J, van Ewijk RJ, Grobbee DE, Painter RC, Uiterwaal CS, Roseboom TJ. Ramadan fasting and newborn's birth weight in pregnant Muslim women in The Netherlands. Br J Nutr. 2014. 112:1503-9 https://www.ncbi.nlm.nih.gov/pubmed/25231606

810 Sayón-Orea C, Bes-Rastrollo M, Carlos S, Beunza JJ, Basterra-Gortari FJ, Martínez-González MA. Association between sleeping hours and siesta and the risk of obesity: The SUN mediterranean cohort. Obesity facts. 2013;6(4):337-47. https://www.ncbi.nlm.nih.gov/pubmed/23970143.

811 Schlogl M, Piaggi P, Pannacciuli N, Bonfiglio SM, Krakoff J, Thearle MS. Energy Expenditure Responses to Fasting and Overfeeding Identify Phenotypes Associated With Weight Change. Diabetes. 2015. 64:3680-9 https://www.ncbi.nlm.nih.gov/pubmed/26185280

\section{Rationale}

Daily Eating Occasions Not Reported

Intervention/Exposure, Daily Eating Occasions Not Reported

Intervention/Exposure, Daily Eating Occasions Not Reported

Intervention/Exposure, Daily Eating Occasions Not Reported, Size of Study Groups

Study Design, Daily Eating

Occasions Not Reported

Daily Eating Occasions Not

Reported, Comparator

Intervention/Exposure

Comparator

Intervention/Exposure, Daily Eating Occasions Not Reported, Outcome

Intervention/Exposure

Intervention/Exposure, Daily Eating Occasions Not Reported 
812 Schnepper R, Richard A, Wilhelm FH, Blechert J. A combined mindfulness-prolonged chewing intervention reduces body weight, food craving, and emotional eating. J Consult Clin Psychol. 2019. 87:106-111 https://www.ncbi.nlm.nih.gov/pubmed/30570305

813 Schuz B, Schuz N, Ferguson SG. It's the power of food: individual differences in food cue responsiveness and snacking in everyday life. Int J Behav Nutr Phys Act. 2015. 12:149 https://www.ncbi.nlm.nih.gov/pubmed/26643690

Intervention/Exposure So

814 Schyns G, van den Akker K, Roefs A, Houben K, Jansen A. Exposure therapy vs lifestyle intervention to reduce food cue reactivity and binge eating in obesity: A pilot study. J Behav Ther Exp Psychiatry. 2019. https://www.ncbi.nlm.nih.gov/pubmed/30732912. \begin{tabular}{ll}
\hline 815 & Seal N. Eating patterns of family with overweight preschool children. Communicating Nursing Research. 2007. \\
$40: 505-505$
\end{tabular}

816 Seker A, Demirci H, Ocakoglu G, Aydin U, Ucar H, Yildiz G, Yaman O. Effect of fasting on 24-h blood pressure values of individuals with no previous history of hypertension. Blood Press Monit. 2017. 22:247-252 https://www.ncbi.nlm.nih.gov/pubmed/28225380

817 Sezen Y, Altiparmak IH, Erkus ME, Kocarslan A, Kaya Z, Gunebakmaz O, Demirbag R. Effects of Ramadan fasting on body composition and arterial stiffness. J Pak Med Assoc. 2016. 66:1522-1527 https://www.ncbi.nlm.nih.gov/pubmed/27924959

818 Shariatpanahi MV, Shariatpanahi ZV, Shahbazi S, Moshtaqi M. Effect of fasting with two meals on BMI and inflammatory markers of metabolic syndrome. Pak J Biol Sci. 2012. 15:255-8 https://www.ncbi.nlm.nih.gov/pubmed/24199461

819 Shariatpanahi ZV, Shariatpanahi MV, Shahbazi S, Hossaini A, Abadi A. Effect of Ramadan fasting on some indices of insulin resistance and components of the metabolic syndrome in healthy male adults. Br J Nutr. 2008. 100:147-51 https://www.ncbi.nlm.nih.gov/pubmed/18053308

820 Shashaj B, Graziani MP, Contoli B, Ciuffo C, Cives C, Facciolini S, Rigoni ML, Spaterna S, Taucci M, Raponi M,
Manco M. Energy Balance-Related Behaviors, Perinatal, Sociodemographic, and Parental Risk Factors Associa with Obesity in Italian Preschoolers. J Am Coll Nutr. 2016. 35:362-71 https://www.ncbi.nlm.nih.gov/pubmed/26933953 after Ramadan. PLoS One. 2012. 7:e47615 https://www.ncbi.nlm.nih.gov/pubmed/23112824

Intervention/Exposure, Daily Eating Occasions Not Reported, Outcome

Intervention/Exposure, Size of Study Groups

Intervention/Exposure, Publication Status

\section{Daily Eating Occasions Not} Reported

Intervention/Exposure, Daily Eating Occasions Not Reported

Daily Eating Occasions Not

Reported, Comparator

Outcome

Study Design

Daily Eating Occasions Not

Reported 
822 Shim E, Ryu HJ, Hwang J, Kim SY, Chung EJ. Dietary sodium intake in young Korean adults and its relationship with eating frequency and taste preference. Nutr Res Pract. 2013. 7:192-8 https://www.ncbi.nlm.nih.gov/pubmed/23766880

823 Shirreffs SM, Maughan RJ. Water and salt balance in young male football players in training during the holy month of Ramadan. J Sports Sci. 2008. 26 Suppl 3:S47-54 https://www.ncbi.nlm.nih.gov/pubmed/19085452

824 Shisslak CM, Mays MZ, Crago M, Jirsak JK, Taitano K, Cagno C. Eating and weight control behaviors among middle school girls in relationship to body weight and ethnicity. J Adolesc Health. 2006. 38:631-3 https://www.ncbi.nlm.nih.gov/pubmed/16635783

825 Short R. Matters of the heart. Nurs Older People. 2006. 18:16-8 https://www.ncbi.nlm.nih.gov/pubmed/17260594

826 Shroff MR, Perng W, Baylin A, Mora-Plazas M, Marin C, Villamor E. Adherence to a snacking dietary pattern and soda intake are related to the development of adiposity: a prospective study in school-age children. Public Health Nutr. 2014. 17:1507-13 https://www.ncbi.nlm.nih.gov/pubmed/23701749

827 Siega-Riz AM, Herrmann TS, Savitz DA, Thorp JM. Frequency of eating during pregnancy and its effect on preterm delivery. American journal of epidemiology. 2001;153(7):647-52. https://www.ncbi.nlm.nih.gov/pubmed/11282791.

828 Siegel AJ, Bhatti NA, Wasfy JH. Reprising Ramadan-Related Angina Pectoris: A Potential Strategy for Risk Reduction. Am J Case Rep. 2016. 17:841-844 https://www.ncbi.nlm.nih.gov/pubmed/27829657

829

Siklova M, Krauzova E, Svobodova B, Kracmerova J, Stepan M, Koc M, Stich V, Rossmeislova L. Circulating Monocyte and Lymphocyte Populations in Healthy First-Degree Relatives of Type 2 Diabetic Patients at Fasting and during Short-Term Hyperinsulinemia. Mediators Inflamm. 2019. 2019:1491083 https://www.ncbi.nlm.nih.gov/pubmed/30983877 Desoye G, Harreiter J, Kautzky-Willer A, Damm P, Mathiesen ER, Jensen DM, Andersen LL, Lapolla A, Dalfra M, Bertolotto A, Wender-Ozegowska E, Zawiejska A, Hill D, Rebollo P, Snoek FJ, van Poppel MN. Results From a European Multicenter Randomized Trial of Physical Activity and/or Healthy Eating to Reduce the Risk of Gestational Diabetes Mellitus: The DALI Lifestyle Pilot. Diabetes Care. 2015. 38:1650-6 https://www.ncbi.nlm.nih.gov/pubmed/26112044

831 Singh RB, Takahashi T, Hristova K, Maheshwari A, Niaz MA, De Meester F, Saboo B, Elkilany G, Srivastav SSL, Srivastav $\mathrm{V}$, etal. Effects of circadian-restricted feeding and low energy intake, on risk of cardiovascular disease and type 2 diabetes mellitus. World heart journal. 2016. 8:315-327
Study Design,

Intervention/Exposure, Outcome

Intervention/Exposure, Daily Eating Occasions Not Reported

Study Design,

Intervention/Exposure

Study Design

Intervention/Exposure

Outcome

Study Design

Intervention/Exposure

Intervention/Exposure

Daily Eating Occasions Not Reported 
832 Sirbu E, Buzas R, Mihaescu R, Suceava I, Lighezan D. Influence of exercise training and eating behavior on arterial stiffness in young healthy students. Wien Klin Wochenschr. 2015. 127:555-60

Daily Eating Occasions Not https://www.ncbi.nlm.nih.gov/pubmed/26033407

833 Siu PMF, Wong SHS, Morris WJ, Chung S. Influence of feeding pattern on postprandial blood glucose responses.

Hong Kong Journal of Sports Medicine \& Sports Science. 2001. 13:52-55

Reported, Comparator

Daily Eating Occasions Not Reported

834 Skipping meals might increase the risk of metabolic syndrome. Nature Clinical Practice Endocrinology and Metabolism. 2008. 4:532-533

Publication Status

835 Skogheim TS, Vollrath ME. Associations of Child Temperament with Child Overweight and Breakfast Habits: A

Population Study in Five-Year-Olds. Nutrients. 2015. 7:10116-28 https://www.ncbi.nlm.nih.gov/pubmed/26633494

Study Design

836 St-Onge MP, Pizinger T, Kovtun K, RoyChoudhury A. Sleep and meal timing influence food intake and its hormonal Comparator regulation in healthy adults with overweight/obesity. Eur J Clin Nutr. 2019;72(Suppl 1):76-82. https://www.ncbi.nlm.nih.gov/pubmed/30487565.

837 Smeets AJ, Westerterp-Plantenga MS. Acute effects on metabolism and appetite profile of one meal difference in the lower range of meal frequency. Br J Nutr. 2008. 99:1316-21 https://www.ncbi.nlm.nih.gov/pubmed/18053311

838 Smith C, Richards R. Dietary intake, overweight status, and perceptions of food insecurity among homeless Minnesotan youth. Am J Hum Biol. 2008. 20:550-63 https://www.ncbi.nlm.nih.gov/pubmed/18491407

\section{Outcome}

$839 \begin{aligned} & \text { Smith DM, Taylor W, Lavender T. Behaviour change techniques to change the postnatal eating and physical activity } \\ & \text { behaviours of women who are obese: a qualitative study. Bjog. 2016. 123:279-84 }\end{aligned}$

https://www.ncbi.nlm.nih.gov/pubmed/26537206

840 Smith KJ, Gall SL, McNaughton SA, Blizzard L, Dwyer T, Venn AJ. Skipping breakfast: longitudinal associations with cardiometabolic risk factors in the Childhood Determinants of Adult Health Study. Am J Clin Nutr. 2010. 92:1316-25 https://www.ncbi.nlm.nih.gov/pubmed/20926520

841 Smith KL, Straker LM, Kerr DA, Smith AJ. Overweight adolescents eat what? And when? Analysis of consumption patterns to guide dietary message development for intervention. J Hum Nutr Diet. 2015. 28 Suppl 2:80-93 https://www.ncbi.nlm.nih.gov/pubmed/25157498 Nutr Diet. 2013. 113:907-19 https://www.ncbi.nlm.nih.gov/pubmed/23647834

\section{Study Design}

\section{Study Design}

\section{Daily Eating Occasions Not} Reported

\section{Study Design,}

Intervention/Exposure, Daily

Eating Occasions Not Reported,

Outcome

\section{Study Design}



adolescents. Scand J Psychol. 2007. 48:23-32 https://www.ncbi.nlm.nih.gov/pubmed/17257366

844 So HK, Nelson EAS, Li AM, Guldan GS, Yin J, Ng PC, et al. Breakfast frequency inversely associated with BMI and body fatness in Hong Kong Chinese children aged 9-18 years. British journal of nutrition. 2011;106(5):742-51. https://www.ncbi.nlm.nih.gov/pubmed/21535905.

845 Soeters MR, Lammers NM, Dubbelhuis PF, Ackermans M, Jonkers-Schuitema CF, Fliers E, Sauerwein HP, Aerts JM, Serlie MJ. Intermittent fasting does not affect whole-body glucose, lipid, or protein metabolism. Am J Clin Nutr. 2009. 90:1244-51 https://www.ncbi.nlm.nih.gov/pubmed/19776143

846 Sok SR, Kim OS, Park MH. Effects of Obesity Management Program Provided by Occupational Health Nurse in Worksite. West J Nurs Res. 2019. 41:728-742 https://www.ncbi.nlm.nih.gov/pubmed/30541407

847 Solah VA, Kerr DA, Hunt WJ, Johnson SK, Boushey CJ, Delp EJ, et al. Effect of Fibre Supplementation on Body Weight and Composition, Frequency of Eating and Dietary Choice in Overweight Individuals. Nutrients. 2017;9(2). https://www.ncbi.nlm.nih.gov/pubmed/28212353.

848 Solianik R, Sujeta A. Two-day fasting evokes stress, but does not affect mood, brain activity, cognitive, psychomotor, and motor performance in overweight women. Behav Brain Res. 2018. 338:166-172 https://www.ncbi.nlm.nih.gov/pubmed/29097329

849 Soreca I, Wallace ML, Hall MH, Hasler BP, Frank E, Kupfer DJ. The association between meal timing and frequency with cardiometabolic profile in patients with bipolar disorder. Acta Psychiatr Scand. 2016. 133:453-8 https://www.ncbi.nlm.nih.gov/pubmed/27084394

850 Souissi N, Souissi H, Sahli S, Tabka Z, Dogui M, Ati J, Davenne D. Effect of Ramadan on the diurnal variation in short-term high power output. Chronobiol Int. 2007. 24:991-1007 https://www.ncbi.nlm.nih.gov/pubmed/17994351

ntervention/Exposure, Daily Eating Occasions Not Reported, Outcome

851 Spaeth AM, Dinges DF, Goel N. Effects of Experimental Sleep Restriction on Weight Gain, Caloric Intake, and Mea Timing in Healthy Adults. Sleep. 2013. 36:981-990 https://www.ncbi.nlm.nih.gov/pubmed/23814334

852 Spaeth AM, Dinges DF, Goel N. Sex and race differences in caloric intake during sleep restriction in healthy adults. Am J Clin Nutr. 2014. 100:559-66 https://www.ncbi.nlm.nih.gov/pubmed/24965304

Intervention/Exposure, Outcome

Daily Eating Occasions Not

Reported

Daily Eating Occasions Not

Reported

Intervention/Exposure

Intervention/Exposure

Study Design, Health Status

Daily Eating Occasions Not Reported 
854 Sriha Belguith A, Baccouche H, Grissa MH, Boubaker H, Bouida W, Beltaief K, Sekma A, Fredj N, Bzeouiche N, Zina Z, Boukef R, Soltani M, Nouira S. The risk of acute coronary syndrome in Ramadan. Tunis Med. 2016. 94:599603 https://www.ncbi.nlm.nih.gov/pubmed/28972251

855 Stange R, Pflugbeil C, Michalsen A, Uehleke B. Therapeutic fasting in patients with metabolic syndrome and impaired insulin resistance. Forsch Komplementmed. 2013. 20:421-6 https://www.ncbi.nlm.nih.gov/pubmed/24434756

856 Stannard SR, Thompson MW, Fairbairn K, Huard B, Sachinwalla T, Thompson CH. Fasting for $72 \mathrm{~h}$ increases intramyocellular lipid content in nondiabetic, physically fit men. Am J Physiol Endocrinol Metab. 2002. 283:E1185-91 https://www.ncbi.nlm.nih.gov/pubmed/12388154

857 Stannard SR, Thompson MW. The effect of participation in Ramadan on substrate selection during submaximal cycling exercise. J Sci Med Sport. 2008. 11:510-7 https://www.ncbi.nlm.nih.gov/pubmed/17706462

858 Stea TH, Vik FN, Bere E, Svendsen MV, Oellingrath IM. Meal pattern among Norwegian primary-school children and longitudinal associations between meal skipping and weight status. Public Health Nutr. 2015. 18:286-91 https://www.ncbi.nlm.nih.gov/pubmed/24521714

859 Stekovic S, Hofer SJ, Tripolt N, Aon MA, Royer P, Pein L, Stadler JT, Pendl T, Prietl B, Url J, Schroeder S, Tadic J, Eisenberg T, Magnes C, Stumpe M, Zuegner E, Bordag N, Riedl R, Schmidt A, Kolesnik E, Verheyen N, Springer A Madl T, Sinner F, de Cabo R, Kroemer G, Obermayer-Pietsch B, Dengjel J, Sourij H, Pieber TR, Madeo F. Alternate Day Fasting Improves Physiological and Molecular Markers of Aging in Healthy, Non-obese Humans. Cell Metab. 2019. 30:462-476.e5 https://www.ncbi.nlm.nih.gov/pubmed/31471173

860 Stote KS, Baer DJ, Spears K, Paul DR, Harris GK, Rumpler WV, Strycula P, Najjar SS, Ferrucci L, Ingram DK, Longo DL, Mattson MP. A controlled trial of reduced meal frequency without caloric restriction in healthy, normalweight, middle-aged adults. Am J Clin Nutr. 2007. 85:981-8 https://www.ncbi.nlm.nih.gov/pubmed/17413096

861 Striegel-Moore RH, Rosselli F, Wilson GT, Perrin N, Harvey K, DeBar L. Nocturnal eating: association with binge eating, obesity, and psychological distress. Int J Eat Disord. 2010. 43:520-6 https://www.ncbi.nlm.nih.gov/pubmed/19708071

862 Stroebele N, McNally J, Plog A, Siegfried S, Hill JO. The association of self-reported sleep, weight status, and academic performance in fifth-grade students. J Sch Health. 2013. 83:77-84 https://www.ncbi.nlm.nih.gov/pubmed/23331266
Sudharsanan N, Romano S, Cunningham SA. School Breakfast Receipt and Obesity among American Fifth- and Eighth-Graders. J Acad Nutr Diet. 2016. 116:599-607.e3 https://www.ncbi.nlm.nih.gov/pubmed/27046061
Language

Intervention/Exposure, Daily Eating Occasions Not Reported

Intervention/Exposure, Daily Eating Occasions Not Reported, Size of Study Groups

Intervention/Exposure, Daily Eating Occasions Not Reported

Daily Eating Occasions Not Reported

Intervention/Exposure, Daily Eating Occasions Not Reported

Eating Frequency Data

Collection

Daily Eating Occasions Not Reported

Study Design

Intervention/Exposure, Daily Eating Occasions Not Reported 
864 Sugimori H, Yoshida K, Izuno T, Miyakawa M, Suka M, Sekine M, Yamagami T, Kagamimori S. Analysis of factors that influence body mass index from ages 3 to 6 years: A study based on the Toyama cohort study. Pediatr Int. 2004. 46:302-10 https://www.ncbi.nlm.nih.gov/pubmed/15151547

865 Sülü B, Öztürk B, Güven A, Kiliç K. The effect of long-term controlled fasting (the Ramadan model) on body mass index, blood biochemistry and oxidative stress factors. Turkiye Klinikleri Journal of Medical Sciences. 2010. 30:855863 http://search.ebscohost.com/login.aspx?direct=true\&db=ilh\&AN=105091038\&site=ehost-live

866 Sundfor TM, Svendsen M, Tonstad S. Effect of intermittent versus continuous energy restriction on weight loss, maintenance and cardiometabolic risk: A randomized 1-year trial. Nutr Metab Cardiovasc Dis. 2018. 28:698-706 https://www.ncbi.nlm.nih.gov/pubmed/29778565

867 Sundfor TM, Tonstad S, Svendsen M. Effects of intermittent versus continuous energy restriction for weight loss on diet quality and eating behavior. A randomized trial. Eur J Clin Nutr. 2019. 73:1006-1014 https://www.ncbi.nlm.nih.gov/pubmed/30514879

868 Supak-Smolcic V, Antoncic D, Ozanic D, Vladilo I, Bilic-Zulle L. Influence of a prolonged fasting and mild activity on routine laboratory tests. Clin Biochem. 2015. 48:85-8 https://www.ncbi.nlm.nih.gov/pubmed/25445731

869 Suriani I, Shamsuddin K, Abdul Latif K, Saad HA. The effect of the Malaysian Food Guideline guidance on a group of overweight and obese women during Ramadan. Saudi Med J. 2015. 36:40-5 https://www.ncbi.nlm.nih.gov/pubmed/25630003

870 Sutton EF, Beyl R, Early KS, Cefalu WT, Ravussin E, Peterson CM. Early Time-Restricted Feeding Improves Insulin Sensitivity, Blood Pressure, and Oxidative Stress Even without Weight Loss in Men with Prediabetes. Cell Metab. 2018. 27:1212-1221.e3 https://www.ncbi.nlm.nih.gov/pubmed/29754952

871 Syam AF. Fasting Can Prevent Various Chronic Diseases. Acta Med Indones. 2016. 48:167-168 https://www.ncbi.nlm.nih.gov/pubmed/27847381

872 Syrad H, Llewellyn CH, Johnson L, Boniface D, Jebb SA, van Jaarsveld CH, Wardle J. Meal size is a critical driver of weight gain in early childhood. Sci Rep. 2016. 6:28368 https://www.ncbi.nlm.nih.gov/pubmed/27321917

$873 \quad$ Taillie LS, Wang D, Popkin BM. Snacking Is Longitudinally Associated with Declines in Body Mass Index z Scores for Overweight Children, but Increases for Underweight Children. J Nutr. 2016. 146:1268-75 https://www.ncbi.nlm.nih.gov/pubmed/27146917
Intervention/Exposure, Daily

Eating Occasions Not Reported

Daily Eating Occasions Not Reported

Daily Eating Occasions Not Reported

Daily Eating Occasions Not Reported

Study Design, Daily Eating Occasions Not Reported, Comparator

Intervention/Exposure, Daily Eating Occasions Not Reported

Daily Eating Occasions Not Reported

Study Design, Publication Status

Age: Intervention/Exposure

Daily Eating Occasions Not Reported

Intervention/Exposure, Daily Eating Occasions Not Reported 

https://www.ncbi.nlm.nih.gov/pubmed/30321490

876 Tan CC, Walczak M, Roach E, Lumeng JC, Miller AL. Longitudinal associations between eating and drinking engagement during mealtime and eating in the absence of hunger in low income toddlers. Appetite. 2018. 130:29-34 https://www.ncbi.nlm.nih.gov/pubmed/30059768

877 Taveras EM, Rifas-Shiman SL, Berkey CS, Rockett HR, Field AE, Frazier AL, et al. Family dinner and adolescent overweight. Obesity research. 2005;13(5):900-6. https://www.ncbi.nlm.nih.gov/pubmed/15919844.

Intervention/Exposure

878 Taylor MA, Garrow JS. Compared with nibbling, neither gorging nor a morning fast affect short-term energy balance in obese patients in a chamber calorimeter. Int J Obes Relat Metab Disord. 2001. 25:519-28 https://www.ncbi.nlm.nih.gov/pubmed/11319656

879 Taylor RW, losua E, Heath AM, Gray AR, Taylor BJ, Lawrence JA, Hanna M, Cameron SL, Sayers R, Galland B. Eating frequency in relation to BMI in very young children: a longitudinal analysis. Public Health Nutr. 2017. 20:1372-1379 https://www.ncbi.nlm.nih.gov/pubmed/28238299

880 Teixeira GP, Barreto ACF, Mota MC, Crispim CA. Caloric midpoint is associated with total calorie and macronutrient intake and body mass index in undergraduate students. Chronobiol Int. 2019. 36:1418-1428 https://www.ncbi.nlm.nih.gov/pubmed/31409139

881 Teixeira GP, Mota MC, Crispim CA. Eveningness is associated with skipping breakfast and poor nutritional intake in Brazilian undergraduate students. Chronobiol Int. 2018. 35:358-367 https://www.ncbi.nlm.nih.gov/pubmed/29219626 effective tool for changing eating habits and body weight among those who practice physical activities. Ciencia \& saude coletiva. 2013;18(2):347-56. https://www.ncbi.nlm.nih.gov/pubmed/23358760.

883 Tejpal S, Sanghera N, Manoharan V, Planas-Iglesias J, Myler K, Klein-Seetharaman J. Towards personalised molecular feedback for weight loss. BMC Obes. 2019. 6:20 https://www.ncbi.nlm.nih.gov/pubmed/31080628

884 Teng NI, Shahar S, Manaf ZA, Das SK, Taha CS, Ngah WZ. Efficacy of fasting calorie restriction on quality of life among aging men. Physiol Behav. 2011. 104:1059-64 https://www.ncbi.nlm.nih.gov/pubmed/21781980

among aging men. Physio/ Behav. 2011. 104:1059-64 https://www.ncbi.nlm.nih.gov/pubmed/21781980
Juul A, Vaag A, Dunger DB. LOW circulating levels of IGF-1 in healthy adults are associated with reduced beta-cell function, increased intramyocellular lipid, and enhanced fat utilization during fasting. J Clin Endocrinol Metab. 2014. 99:2198-207 https://www.ncbi.nlm.nih.gov/pubmed/24617714

Intervention/Exposure, Daily Eating Occasions Not Reported

Outcome

\section{Eating Frequency Data}

Collection

Study Design,

Intervention/Exposure, Outcome

\section{Study Design, Outcome}

Language

Daily Eating Occasions Not Reported, Comparator

Intervention/Exposure, Daily Eating Occasions Not Reported

Intervention/Exposure, Daily Eating Occasions Not Reported 
886 Thiara B.

Cardiovascular disease. Nurs Stand. 2015. 29:60 https://www.ncbi.nlm.nih.gov/pubmed/25872853

Study Design

Intervention/Exposure

887 Thivel D, Finlayson G, Miguet M, Pereira B, Duclos M, Boirie Y, Doucet E, Blundell JE, Metz L. Energy depletion by 24-h fast leads to compensatory appetite responses compared with matched energy depletion by exercise in healthy young males. Br J Nutr. 2018. 120:583-592 https://www.ncbi.nlm.nih.gov/pubmed/30058508

888 Thomas EA, Higgins J, Bessesen DH, McNair B, Cornier MA. Usual breakfast eating habits affect response to breakfast skipping in overweight women. Obesity. 2015. 23:750-9 https://www.ncbi.nlm.nih.gov/pubmed/25755093

889 Timlin MT, Pereira MA, Story M, Neumark-Sztainer D. Breakfast eating and weight change in a 5-year prospective analysis of adolescents: Project EAT (Eating Among Teens). Pediatrics. 2008. 121:e638-45 https://www.ncbi.nlm.nih.gov/pubmed/18310183

890 Timlin MT, Pereira MA. Breakfast frequency and quality in the etiology of adult obesity and chronic diseases. Nutrition reviews. 2007;65(6):268-81. https://www.ncbi.nlm.nih.gov/pubmed/17605303.

891 Timmermans M, Mackenbach JD, Charreire H, Bardos H, Compernolle S, De Bourdeaudhuij I, Oppert JM, Rutter H, McKee M, Lakerveld J. Exploring the mediating role of energy balance-related behaviours in the association between sleep duration and obesity in European adults. The SPOTLIGHT project. Prev Med. 2017. 100:25-32 https://www.ncbi.nlm.nih.gov/pubmed/28359703

892 Tin SP, Ho SY, Mak KH, Wan KL, Lam TH. Breakfast skipping and change in body mass index in young children. Int J Obes. 2011. 35:899-906 https://www.ncbi.nlm.nih.gov/pubmed/21448130

Daily Eating Occasions Not Reported, Size of Study Groups

Daily Eating Occasions Not Reported

Daily Eating Occasions Not

Reported

Study Design

Study Design, Daily Eating Occasions Not Reported

Daily Eating Occasions Not Reported

Study Design

893 Tin SP, Ho SY, Mak KH, Wan KL, Lam TH. Lifestyle and socioeconomic correlates of breakfast skipping in Hong Kong primary 4 schoolchildren. Prev Med. 2011. 52:250-3 https://www.ncbi.nlm.nih.gov/pubmed/21215276 Tin SP, Ho SY, Mak KH, Wan KL, Lam TH. Location of breakfast consumption predicts body mass index change in
young Hong Kong children. Int J Obes. 2012. 36:925-30 https://www.ncbi.nlm.nih.gov/pubmed/22234278

895 Tinsley GM, Forsse JS, Butler NK, Paoli A, Bane AA, La Bounty PM, Morgan GB, Grandjean PW. Time-restricted feeding in young men performing resistance training: A randomized controlled trial. Eur J Sport Sci. 2017. 17:200207 https://www.ncbi.nlm.nih.gov/pubmed/27550719

896 Tinsley GM, Moore ML, Graybeal AJ, Paoli A, Kim Y, Gonzales JU, Harry JR, VanDusseldorp TA, Kennedy DN, Cruz MR. Time-restricted feeding plus resistance training in active females: a randomized trial. Am J Clin Nutr. 2019. 110:628-640 https://www.ncbi.nlm.nih.gov/pubmed/31268131

Intervention/Exposure

Daily Eating Occasions Not Reported

Intervention/Exposure 
897 Tinsley GM, Moore ML, Graybeal AJ. Reliability of hunger-related assessments during 24-hour fasts and their relationship to body composition and subsequent energy compensation. Physiol Behav. 2018. 188:221-226

Daily Eating Occasions Not https://www.ncbi.nlm.nih.gov/pubmed/29438660

898 Topacoglu H, Karcioglu O, Yuruktumen A, Kiran S, Cimrin AH, Ozucelik DN, Sarikaya S, Soysal S, Turpcu U, Bozkurt S. Impact of Ramadan on demographics and frequencies of disease-related visits in the emergency department. Int J Clin Pract. 2005. 59:900-5 https://www.ncbi.nlm.nih.gov/pubmed/16033610

899 Torbahn G, Gellhaus I, Koch B, von Kries R, Obermeier V, Holl RW, Fink K, van Egmond-Frohlich A. Reduction of Portion Size and Eating Rate Is Associated with BMI-SDS Reduction in Overweight and Obese Children and Adolescents: Results on Eating and Nutrition Behaviour from the Observational KgAS Study. Obes Facts. 2017. 10:503-516 https://www.ncbi.nlm.nih.gov/pubmed/29084405

900 Torjesen I. The Finland experience: leading the way in prevention. Circulation. 2007. 115:f11-2 https://www.ncbi.nlm.nih.gov/pubmed/17245844

901 Toschke AM, Kuchenhoff H, Koletzko B, von Kries R. Meal frequency and childhood obesity. Obes Res. 2005. 13:1932-8 https://www.ncbi.nlm.nih.gov/pubmed/16339125

902 Toschke AM, Thorsteinsdottir KH, von Kries R. Meal frequency Toschke AM, Thorsteinsdottir KH, von Kries R. Meal frequency, breakfast consun
Pediatr Obes. 2009. 4:242-8 https://www.ncbi.nlm.nih.gov/pubmed/19922038

903

Toyoshima H, Masuoka N, Hashimoto S, Otsuka R, Sasaki S, Tamakoshi K, Yatsuya H. Effect of the interaction between mental stress and eating pattern on body mass index gain in healthy Japanese male workers. J Epidemiol. 2009. 19:88-93 https://www.ncbi.nlm.nih.gov/pubmed/19265270

904 Trabelsi K, el Abed K, Stannard SR, Jammoussi K, Zeghal KM, Hakim A. Effects of fed- versus fasted-state aerobic training during Ramadan on body composition and some metabolic parameters in physically active men. Int $J$ Sport Nutr Exerc Metab. 2012. 22:11-8 https://www.ncbi.nlm.nih.gov/pubmed/22248495 Biochemical and Anthropometric Parameters in Physically Active Men. Asian Journal of Sports Medicine. 2011;2(3):134-44. https://www.ncbi.nlm.nih.gov/pubmed/22375232.

Reported, Size of Study Groups

Daily Eating Occasions Not Reported

Intervention/Exposure $x^{2}$

\section{Study Design}

Study Design

Study Design

Intervention/Exposure

(1)

Daily Eating Occasions Not

Reported

\section{Daily Eating Occasions Not} Reported

Daily Eating Occasions Not Reported 
907 Traub M, Lauer R, Kesztyus T, Wartha O, Steinacker JM, Kesztyus D. Skipping breakfast, overconsumption of soft drinks and screen media: longitudinal analysis of the combined influence on weight development in primary

Daily Eating Occasions Not schoolchildren. BMC Public Health. 2018. 18:363 https://www.ncbi.nlm.nih.gov/pubmed/29548323

908 Tremblay A, Lepage C, Panahi S, Couture C, Drapeau V. Adaptations to a diet-based weight-reducing programme

in obese women resistant to weight loss. Clin Obes. 2015. 5:145-53 https://www.ncbi.nlm.nih.gov/pubmed/25872975

Reported

Intervention/Exposure, Daily Eating Occasions Not Reported

909 Trepanowski J, Kroeger C, Barnosky A, Hoddy K, Varady K. Alternateday fasting and daily calorie restriction similarly affect visceral adiposity and circulating inflammatory cytokine concentrations. FASEB journal.

Study Design, Publication Status 2015;29(Suppl. 1).

910 Trepanowski J, Kroeger C, Klempel M, Calvo Y, Varady K. Alternateday fasting versus daily calorie restriction for weight loss and cardio-protection. FASEB journal. 2014;28(Suppl. 1).

911 Trepanowski JF, Kroeger CM, Barnosky A, Klempel M, Bhutani S, Hoddy KK, Rood J, Ravussin E, Varady KA Effects of alternate-day fasting or daily calorie restriction on body composition, fat distribution, and circulating adipokines: Secondary analysis of a randomized controlled trial. Clin Nutr. 2018. 37:1871-1878 https://www.ncbi.nlm.nih.gov/pubmed/29258678

912 Trepanowski JF, Kroeger CM, Barnosky A, Klempel MC, Bhutani S, Hoddy KK, Gabel K, Freels S, Rigdon J, Rood J, Ravussin E, Varady KA. Effect of Alternate-Day Fasting on Weight Loss, Weight Maintenance, and Cardioprotection Among Metabolically Healthy Obese Adults: A Randomized Clinical Trial. JAMA Intern Med. 2017. 177:930-938 https://www.ncbi.nlm.nih.gov/pubmed/28459931

913 Trepanowski JF, Kabir MM, Alleman Jr RJ, Bloomer RJ. A 21-day Daniel fast with or without krill oil supplementation improves anthropometric parameters and the cardiometabolic profile in men and women. Nutrition \& metabolism. 2012;9(1):82-8. https://www.ncbi.nlm.nih.gov/pubmed/22971786.

914 Trotti R, Rondanelli M, Cuzzoni G, Ferrari E, d'Eril GM. Circadian temporal organization of lipidic fractions in elderly Study Design people. Entrainment to the dietary schedule. Aging Clin Exp Res. 2002. 14:94-9 https://www.ncbi.nlm.nih.gov/pubmed/12092790

915 Tucker JM, DeFrang R, Orth J, Wakefield S, Howard K. Evaluation of a Primary Care Weight Management Program in Children Aged 2(-)5 years: Changes in Feeding Practices, Health Behaviors, and Body Mass Index. Nutrients. 2019;11(3). https://www.ncbi.nlm.nih.gov/pubmed/30818772. activity, nutritional and food safety knowledge and beliefs in an adolescent Italian population. J Am Coll Nutr. 2008. 27:31-43 https://www.ncbi.nlm.nih.gov/pubmed/18460479

Daily Eating Occasions Not Reported, Size of Study Groups

Intervention/Exposure, Daily Eating Occasions Not Reported,

Comparator

Intervention/Exposure, Comparator

Intervention/Exposure

\section{Study Design}


917 Turner-McGrievy GM, Dunn CG, Wilcox S, Boutte AK, Hutto B, Hoover A, Muth E. Defining Adherence to Mobile Dietary Self-Monitoring and Assessing Tracking Over Time: Tracking at Least Two Eating Occasions per Day Is Best Marker of Adherence within Two Different Mobile Health Randomized Weight Loss Interventions. J Acad Nutr Diet. 2019. 119:1516-1524 https://www.ncbi.nlm.nih.gov/pubmed/31155473

918 Uemura M, Yatsuya H, Hilawe EH, Li Y, Wang C, Chiang C, Otsuka R, Toyoshima H, Tamakoshi K, Aoyama A. Breakfast Skipping is Positively Associated With Incidence of Type 2 Diabetes Mellitus: Evidence From the Aichi Workers' Cohort Study. J Epidemiol. 2015. 25:351-8 https://www.ncbi.nlm.nih.gov/pubmed/25787236

919 Unalacak M, Kara IH, Baltaci D, Erdem O, Bucaktepe PG. Effects of Ramadan fasting on biochemical and hematological parameters and cytokines in healthy and obese individuals. Metab Syndr Relat Disord. 2011. 9:15761 https://www.ncbi.nlm.nih.gov/pubmed/21235381 people: relationships with eating behaviors and body mass index. J Nutr Educ Behav. 2013. 45:3-11 https://www.ncbi.nlm.nih.gov/pubmed/23110750

921 Uusitalo U, Arkkola T, Ovaskainen ML, Kronberg-Kippila C, Kenward MG, Veijola R, Simell O, Knip M, Virtanen SM. Unhealthy dietary patterns are associated with weight gain during pregnancy among Finnish women. Public Health Nutr. 2009. 12:2392-9 https://www.ncbi.nlm.nih.gov/pubmed/19323867

922 Vaitkeviciute J, Petrauskiene A. The Associations between Body Mass Index of Seven- and Eight-Year-Old Children, Dietary Behaviour and Nutrition-Related Parenting Practices. Medicina. 2019;55(1). https://www.ncbi.nlm.nih.gov/pubmed/30669687.

923 van den Berg P, Neumark-Sztainer D, Hannan PJ, Haines J. Is dieting advice from magazines helpful or harmful? Five-year associations with weight-control behaviors and psychological outcomes in adolescents. Pediatrics. 2007. 119:e30-7 https://www.ncbi.nlm.nih.gov/pubmed/17200254

924 van der Crabben SN, Allick G, Ackermans MT, Endert E, Romijn JA, Sauerwein HP. Prolonged fasting induces peripheral insulin resistance, which is not ameliorated by high-dose salicylate. J Clin Endocrinol Metab. 2008. 93:638-41 https://www.ncbi.nlm.nih.gov/pubmed/18056775

925 van der Wijden CL, Steinbach S, van der Ploeg HP, van Mechelen W, van Poppel MN. A longitudinal study on the relationship between eating style and gestational weight gain. Appetite. 2014. 83:304-8 https://www.ncbi.nlm.nih.gov/pubmed/25218880

Daily Eating Occasions Not

Reported

Daily Eating Occasions Not Reported

Study Design

Intervention/Exposure

Study Design

Intervention/Exposure

Intervention/Exposure

Intervention/Exposure

Daily Eating Occasions Not

Reported, Outcome 
927 van Ewijk RJ, Painter RC, Roseboom TJ. Associations of prenatal exposure to Ramadan with small stature and thinness in adulthood: results from a large Indonesian population-based study. Am J Epidemiol. 2013. 177:729-36

Intervention/Exposure, Daily https://www.ncbi.nlm.nih.gov/pubmed/23486307

928 van Herpen NA, Sell H, Eckel J, Schrauwen P, Mensink RP. Prolonged fasting and the effects on biomarkers of inflammation and on adipokines in healthy lean men. Horm Metab Res. 2013. 45:378-82 https://www.ncbi.nlm.nih.gov/pubmed/23235922

929 Van Tine ML, McNicholas F, Safer DL, Agras WS. Follow-up of selective eaters from childhood to adulthood. Eat Behav. 2017. 26:61-65 https://www.ncbi.nlm.nih.gov/pubmed/28152419

930 van Veen MR, van Hasselt PM, de Sain-van der Velden MG, Verhoeven N, Hofstede FC, de Koning TJ, Visser G. Metabolic profiles in children during fasting. Pediatrics. 2011. 127:e1021-7 https://www.ncbi.nlm.nih.gov/pubmed/21422093

931 Vander Wal JS, Waller SM, Klurfeld DM, McBurney MI, Cho S, Kapila M, Dhurandhar NV. Effect of a post-dinner snack and partial meal replacement program on weight loss. Int J Food Sci Nutr. 2006. 57:97-106 https://www.ncbi.nlm.nih.gov/pubmed/16849118

\section{2} Vandeweghe L, Verbeken S, Vervoort L, Moens E, Braet C. Reward sensitivity and body weight: the intervening role of food responsive behavior and external eating. Appetite. 2017. 112:150-156 https://www.ncbi.nlm.nih.gov/pubmed/28108344

933 Vanelli M, lovane B, Bernardini A, Chiari G, Errico MK, Gelmetti C, Corchia M, Ruggerini A, Volta E, Rossetti S. Breakfast habits of 1,202 northern Italian children admitted to a summer sport school. Breakfast skipping is associated with overweight and obesity. Acta Biomed. 2005. 76:79-85 https://www.ncbi.nlm.nih.gov/pubmed/16350552

\section{4}

Vanelli M, Monti G, Volta E, Finestrella V, Gkliati D, Cangelosi M, Caragnulo R, Vitale M, Ingrosso L, Scazzina F. "GIOCAMPUS" - An effective school-based intervention for breakfast promotion and overweight risk reduction. Acta Biomed. 2014. 84:181-8 https://www.ncbi.nlm.nih.gov/pubmed/24458162

$935 \quad$ Varady K. Alternate-day fasting. Menopause. 2016. 23:1368

936 Varady KA, Bhutani S, Church EC, Klempel MC. Short-term modified alternate-day fasting: a novel dietary strategy for weight loss and cardioprotection in obese adults. Am J Clin Nutr. 2009. 90:1138-43 https://www.ncbi.nlm.nih.gov/pubmed/19793855

Daily Eating Occasions Not Reported

Intervention/Exposure

Daily Eating Occasions Not Reported

Daily Eating Occasions Not Reported

Study Design

Study Design, Daily Eating

Occasions Not Reported

Daily Eating Occasions Not

Reported

Publication Status

Study Design, Daily Eating

Occasions Not Reported

Daily Eating Occasions Not Reported, Comparator 

alternate day modified fasting in obese adults. Br J Nutr. 2011. 105:580-3 https://www.ncbi.nlm.nih.gov/pubmed/20880415

939 Varady KA, Dam VT, Klempel MC, Horne M, Cruz R, Kroeger CM, Santosa S. Corrigendum: Effects of weight loss via high fat vs. low fat alternate day fasting diets on free fatty acid profiles. Sci Rep. 2015. 5:8806

Study Design, Publication Status https://www.ncbi.nlm.nih.gov/pubmed/26244425

940 Varady KA, Dam VT, Klempel MC, Horne M, Cruz R, Kroeger CM, Santosa S. Effects of weight loss via high fat vs. low fat alternate day fasting diets on free fatty acid profiles. Sci Rep. 2015. 5:7561 https://www.ncbi.nlm.nih.gov/pubmed/25557754

941 Varady KA, Gabel K. Dietary adherence and macronutrient intake during 12 months of alternate day fasting. FASEB Study Design, Publication Status journal. 2017;31(1).

942 Varady KA, Hoddy KK, Kroeger CM, Trepanowski JF, Klempel MC, Barnosky A, Bhutani S. Determinants of weight Intervention/Exposure loss success with alternate day fasting. Obes Res Clin Pract. 2016. 10:476-80 https://www.ncbi.nlm.nih.gov/pubmed/26385599

943 Vardarli MC, Hammes HP, Vardarli I. Possible metabolic impact of Ramadan fasting in healthy men. Turk J Med Sci. 2014. 44:1010-20 https://www.ncbi.nlm.nih.gov/pubmed/25552155

944 Vatanparast H, Islam N, Patil RP, Shafiee M, Smith J, Whiting S. Snack Consumption Patterns among Canadians. Study Design Nutrients. 2019;11(5). https://www.ncbi.nlm.nih.gov/pubmed/31126080.

945 Veloso SusanaM, Matos MargaridaG, Marina Carvalho, Diniz JoséA. Psychosocial Factors of Different Health Behaviour Patterns in Adolescents: Association with Overweight and Weight Control Behaviours. Journal of Obesity. 2012. 2012:1-10 http://search.ebscohost.com/login.aspx?direct=true\&db=jlh\&AN=104278012\&site=ehost-live

946 Veltsista A, Laitinen J, Sovio U, Roma E, Jarvelin MR, Bakoula C. Relationship between eating behavior, breakfast consumption, and obesity among Finnish and Greek adolescents. J Nutr Educ Behav. 2010. 42:417-21 https://www.ncbi.nlm.nih.gov/pubmed/20729150 Fasting increases human skeletal muscle net phenylalanine release and this is associated with decreased mTOR signaling. PLoS One. 2014. 9:e102031 https://www.ncbi.nlm.nih.gov/pubmed/25020061

Intervention/Exposure, Daily Eating Occasions Not Reported

ntervention/Exposure, Daily Eating Occasions Not Reported

Study Design

Study Design

Intervention/Exposure, Daily Eating Occasions Not Reported

Study Design

Vergetaki A, Linardakis M, Papadaki A, Kafatos A. Presence of metabolic syndrome and cardiovascular risk factors in adolescents and University students in Crete (Greece), according to different levels of snack consumption. Appetite. 2011. 57:278-85 https://www.ncbi.nlm.nih.gov/pubmed/21640150 


\section{Citation}

949

Verhoef SP, Camps SG, Gonnissen HK, Westerterp KR, Westerterp-Plantenga MS. Concomitant changes in sleep duration and body weight and body composition during weight loss and 3-mo weight maintenance. Am J Clin Nutr. 2013. 98:25-31 https://www.ncbi.nlm.nih.gov/pubmed/23697706

950 Versteeg RI, Ackermans MT, Nederveen AJ, Fliers E, Serlie MJ, la Fleur SE. Meal timing effects on insulin sensitivity and intrahepatic triglycerides during weight loss. Int J Obes. 2018. 42:156-162 https://www.ncbi.nlm.nih.gov/pubmed/28811653

$951 \quad$ Versteeg RI, Schrantee A, Adriaanse SM, Unmehopa UA, Booij J, Reneman L, Fliers E, la Fleur SE, Serlie MJ. Timing of caloric intake during weight loss differentially affects striatal dopamine transporter and thalamic serotonin transporter binding. Faseb j. 2017. 31:4545-4554 https://www.ncbi.nlm.nih.gov/pubmed/28679529

952 Veugelers PJ, Fitzgerald AL. Prevalence of and risk factors for childhood overweight and obesity. CMAJ: Canadian Medical Association Journal. 2005;173(6):607-13. https://www.ncbi.nlm.nih.gov/pubmed/16157724.

953 Vieira Musse GN, Moreira T, Ayumi Kimura M, Pereira FWL, Okoshi K, Garcia Zanati S, et al. Skipping breakfast concomitant with late-night dinner eating is associated with worse outcomes following ST-segment elevation myocardial infarction. Eur J Prev Cardiol. 2019:2047487319839546. https://www.ncbi.nlm.nih.gov/pubmed/30995859.

\section{4}

Vieten C, Laraia BA, Kristeller J, Adler N, Coleman-Phox K, Bush NR, Wahbeh H, Duncan LG, Epel E. The mindful moms training: development of a mindfulness-based intervention to reduce stress and overeating during pregnancy. BMC Pregnancy Childbirth. 2018. 18:201 https://www.ncbi.n/m.nih.gov/pubmed/29859038

Vigna L, Brunani A, Brugnera A, Grossi E, Compare A, Tirelli AS, Conti DM, Agnelli GM, Andersen LL, Buscema M, Riboldi L. Determinants of metabolic syndrome in obese workers: gender differences in perceived job-related stress and in psychological characteristics identified using artificial neural networks. Eat Weight Disord. 2019. 24:73-81 https://www.ncbi.nlm.nih.gov/pubmed/29987776

Vik FN, Overby NC, Lien N, Bere E. Number of meals eaten in relation to weight status among Norwegian adolescents. Scand J Public Health. 2010. 38:13-8 https://www.ncbi.nlm.nih.gov/pubmed/21062835

957 Vilela S, Oliveira A, Severo M, Lopes C. Chrono-Nutrition: The Relationship between Time-of-Day Energy and Macronutrient Intake and Children's Body Weight Status. J Biol Rhythms. 2019. 34:332-342 https://www.ncbi.nlm.nih.gov/pubmed/30917726
Vinales KL, Schlogl M, Reinhardt M, Thearle MS, Krakoff J, Piaggi P. Cycling Efficiency During Incremental Cycle Ergometry After 24 Hours of Overfeeding or Fasting. Obesity. 2018. 26:368-377 https://www.ncbi.nlm.nih.gov/pubmed/29276860

\section{Rationale}

Intervention/Exposure, Daily

Eating Occasions Not Reported

Comparator

ntervention/Exposure, Daily

Eating Occasions Not Reported,

Comparator

Study Design

Study Design, Publication Status, Health Status

Intervention/Exposure

Intervention/Exposure

\section{Study Design}

Daily Eating Occasions Not Reported

Intervention/Exposure, Size of Study Groups 

Diabetic medicine. 2014;31(11):1323-30. https://www.ncbi.nlm.nih.gov/pubmed/24989831.

960 Viskaal-van Dongen M, Kok FJ, de Graaf C. Effects of snack consumption for 8 weeks on energy intake and body weight. Int J Obes. 2010. 34:319-26 https://www.ncbi.nlm.nih.gov/pubmed/19935746

Eating Frequency Data Collection

961 von Katzler R, Zyriax BC, Jagemann B, Westenhoefer J, Jensen HJ, Harth V, Oldenburg M. Lifestyle behaviour and prevalence of cardiovascular risk factors - a pilot study comparing Kiribati and European seafarers. BMC Public Health. 2019. 19:855 https://www.ncbi.nlm.nih.gov/pubmed/31262273

962 Wagner R, Oberste-Berghaus C, Herpertz S, Blum WF, Pelz B, Hebebrand J, Senf W, Mann K, Albers N. Time relationship between circadian variation of serum levels of leptin, insulin and cortisol in healthy subjects. Horm Res. 2000. 54:174-80 https://www.ncbi.nlm.nih.gov/pubmed/11416234 hyperandrogenism in lean women with polycystic ovary syndrome. Diabetologia. 2012. 55:S235-S236

\title{
Intervention/Exposure
}

Intervention/Exposure, Daily

Eating Occasions Not Reported

964 Waller CE, Du S, Popkin BM. Patterns of overweight, inactivity, and snacking in Chinese children. Obes Res. 2003. 11:957-61 https://www.ncbi.nlm.nih.gov/pubmed/12917500

965 Waller SM, Vander Wal JS, Klurfeld DM, McBurney MI, Cho S, Bijlani S, Dhurandhar NV. Evening ready-to-eat cereal consumption contributes to weight management. J Am Coll Nutr. 2004. 23:316-21 https://www.ncbi.nlm.nih.gov/pubmed/15310735

966 Wang P, Lu MC, Yu CW, Yan YH. Influence of the time of day and fasting duration on glucose level following a 1hour, 50-gram glucose challenge test in pregnant women. PLoS One. 2014. 9:e112526 https://www.ncbi.nlm.nih.gov/pubmed/25393415

967 Wang S, Schwartz MB, Shebl FM, Read M, Henderson KE, Ickovics JR. School breakfast and body mass index: a longitudinal observational study of middle school students. Pediatr Obes. 2017. 12:213-220 https://www.ncbi.nlm.nih.gov/pubmed/26989876

968 Washburn RL, Cox JE, Muhlestein JB, May HT, Carlquist JF, Le VT, et al. Pilot Study of Novel Intermittent Fasting Effects on Metabolomic and Trimethylamine N-oxide Changes During 24-hour Water-Only Fasting in the FEELGOOD Trial. Nutrients. 2019;11(2). https://www.ncbi.nlm.nih.gov/pubmed/30678028.

\section{Health Status}

Intervention/Exposure, Daily

Eating Occasions Not Reported

Intervention/Exposure, Daily Eating Occasions Not Reported

Daily Eating Occasions Not Reported

Daily Eating Occasions Not Reported, Outcome Watanabe Y, Saito I, Henmi I, Yoshimura K, Maruyama K, Yamauchi K, Matsuo T, Kato T, Tanigawa T, Kishida T, Asada Y. Skipping Breakfast is Correlated with Obesity. J Rural Med. 2014. 9:51-8 https://www.ncbi.nlm.nih.gov/pubmed/25648986

.

hes.//WwW.ncbinlm.nih.gov/pubmed/25648986


970 Webb JB, Hardin AS. A preliminary evaluation of BMI status in moderating changes in body composition and eating

Intervention/Exposure behavior in ethnically-diverse first-year college women. Eat Behav. 2012. 13:402-5 https://www.ncbi.nlm.nih.gov/pubmed/23121798

971 Wegman MP, Guo MH, Bennion DM, Shankar MN, Chrzanowski SM, Goldberg LA, Xu J, Williams TA, Lu X, Hsu SI, Anton SD, Leeuwenburgh C, Brantly ML. Practicality of intermittent fasting in humans and its effect on oxidative stress and genes related to aging and metabolism. Rejuvenation Res. 2015. 18:162-72 https://www.ncbi.nlm.nih.gov/pubmed/25546413

972 Wehrens SMT, Christou S, Isherwood C, Middleton B, Gibbs MA, Archer SN, Skene DJ, Johnston JD. Meal Timing Regulates the Human Circadian System. Curr Biol. 2017. 27:1768-1775.e3 https://www.ncbi.nlm.nih.gov/pubmed/28578930

Intervention/Exposure, Daily Eating Occasions Not Reported

973 Wei M, Brandhorst S, Shelehchi M, Mirzaei H, Cheng CW, Budniak J, et al. Fasting-mimicking diet and markers/risk factors for aging, diabetes, cancer, and cardiovascular disease. Sci Transl Med. 2017;9(377). https://www.ncbi.nlm.nih.gov/pubmed/28202779.

974 Wengreen HJ, Moncur C. Change in diet, physical activity, and body weight among young-adults during the transition from high school to college. Nutr J. 2009. 8:32 https://www.ncbi.nlm.nih.gov/pubmed/19624820

Intervention/Exposure, Daily Eating Occasions Not Reported, Comparator

Daily Eating Occasions Not Reported

Wennberg M, Gustafsson PE, Wennberg P, Hammarstrom A. Irregular eating of meals in adolescence and the metabolic syndrome in adulthood: results from a 27-year prospective cohort. Public Health Nutr. 2016. 19:667-73

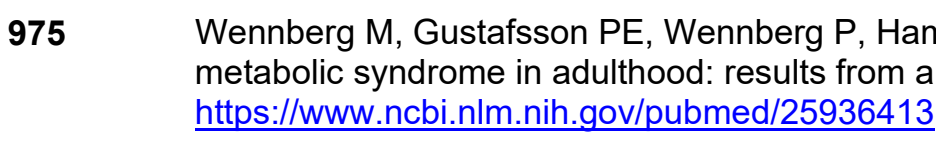

976 Wennberg M, Gustafsson PE, Wennberg P, Hammarstrom A. Poor breakfast habits in adolescence predict the metabolic syndrome in adulthood. Public Health Nutr. 2015. 18:122-9 https://www.ncbi.nlm.nih.gov/pubmed/24468205

Werneck AO, Agostinete RR, Cayres SU, Urban JB, Wigna A, Chagas LGM, et al. Association between Cluster of
Lifestyle Behaviors and HOMA-IR among Adolescents: ABCD Growth Study. Medicina. 2018;54(6). https://www.ncbi.nlm.nih.gov/pubmed/30513771.

978 Westenhoefer J, von Falck B, Stellfeldt A, Fintelmann S. Behavioural correlates of successful weight reduction over 3 y. Results from the Lean Habits Study. Int J Obes Relat Metab Disord. 2004. 28:334-5 https://www.ncbi.nlm.nih.gov/pubmed/14647175

\section{Daily Eating Occasions Not} Reported

\section{Study Design}

Intervention/Exposure, Daily Eating Occasions Not Reported

\section{Study Design}



partially temporally isolated men. Int J Obes Relat Metab Disord. 2002. 26:102-10 https://www.ncbi.nlm.nih.gov/pubmed/11791154

981 White MA, Martin PD, Newton RL, Walden HM, York-Crowe EE, Gordon ST, Ryan DH, Williamson DA. Mediators of weight loss in a family-based intervention presented over the internet. Obes Res. 2004. 12:1050-9

Intervention/Exposure, Daily https://www.ncbi.nlm.nih.gov/pubmed/15292468

\section{2} Whitelock V, Kersbergen I, Higgs S, Aveyard P, Halford JCG, Robinson E. A smartphone based attentive eating intervention for energy intake and weight loss: results from a randomised controlled trial. BMC Public Health. 2019. 19:611 https://www.ncbi.nlm.nih.gov/pubmed/31113400

983 Widhalm K, Pöppelmeyer C, Helk O. The Effect of Alternate-Day Fasting (ADF) on Weight Loss, Metabolic Parameters and Psychological Characteristics. Aktuelle ernahrungsmedizin. 2017;42(3):188-92.

\section{4}

Widome R, Neumark-Sztainer D, Hannan PJ, Haines J, Story M. Eating when there is not enough to eat: eating behaviors and perceptions of food among food-insecure youths. Am J Public Health. 2009. 99:822-8 https://www.ncbi.nlm.nih.gov/pubmed/19299675

985 Wijngaarden MA, van der Zon GC, van Dijk KW, Pijl H, Guigas B. Effects of prolonged fasting on AMPK signaling, gene expression, and mitochondrial respiratory chain content in skeletal muscle from lean and obese individuals. Am J Physiol Endocrinol Metab. 2013. 304:E1012-21 https://www.ncbi.nlm.nih.gov/pubmed/23512807

986 Wijtzes Al, Jansen W, Bouthoorn SH, van Lenthe FJ, Franco OH, Hofman A, Jaddoe VW, Raat H. Meal-Skipping Behaviors and Body Fat in 6-Year-Old Children. J Pediatr. 2016. 168:118-25.e2 https://www.ncbi.nlm.nih.gov/pubmed/26520914

987 Witbracht M, Keim NL, Forester S, Widaman A, Laugero K. Female breakfast skippers display a disrupted cortisol rhythm and elevated blood pressure. Physiol Behav. 2015. 140:215-21 https://www.ncbi.nlm.nih.gov/pubmed/25545767

988 Wong T, Hirahatake K, Nguyen T, Carlos AC, Odegaard AO. The effect of a time restricted eating approach vs. standard heart healthy dietary counseling on cardiometabolic health measures in adults with abdominal obesity: a randomized pilot trial. Circulation. 2019;139.

\section{Study Design}

Intervention/Exposure, Daily Eating Occasions Not Reported

Daily Eating Occasions Not Reported

Study Design, Daily Eating Occasions Not Reported Study Design, Publication Status Study Design 

between food insecurity and obesity among socioeconomically disadvantaged youth. Appetite. 2019. 132:275-281 https://www.ncbi.nlm.nih.gov/pubmed/30327150

991 Wuenstel JW, Kowalkowska J, Wadolowska L, Slowinska MA, Niedzwiedzka E, Kurp L. Habitual eating of breakfast, consumption frequency of selected food and overweight prevalence in adolescents from various age groups. Dev

Study Design, Daily Eating Period Med. 2015. 19:193-201 https://www.ncbi.nlm.nih.gov/pubmed/26384123

\section{2} Wylie J, Barr S, Jeanes Y. Eating frequency and sna of Human Nutrition \& Dietetics. 2009. 22:274-275

993 Wynn P, Wynn J. Smairt Snacking BYPASS JUNK FOOD AND PICK HEALTHY GO-TO SNACKS. Diabetes Self Manag. 2017. 34:70-71 https://www.ncbi.nlm.nih.gov/pubmed/29714853

994 Xiao Q, Garaulet M, Fajl Scheer. Meal timing and obesity: interactions with macronutrient intake and chronotype. Int J Obes (Lond). 2019. 43:1701-1711 https://www.ncbi.nlm.nih.gov/pubmed/30705391

995 Yamane M, Ekuni D, Mizutani S, Kataoka K, Sakumoto-Kataoka M, Kawabata Y, Omori C, Azuma T, Tomofuji T, Iwasaki Y, Morita M. Relationships between eating quickly and weight gain in Japanese university students: a longitudinal study. Obesity (Silver Spring). 2014. 22:2262-6 https://www.ncbi.nlm.nih.gov/pubmed/25044853 Yannakoulia M, Ntalla I, Papoutsakis C, Farmaki AE, Dedoussis GV. Consumption of vegetables, cooked meals, and eating dinner is negatively associated with overweight status in children. J Pediatr. 2010. 157:815-20 https://www.ncbi.nlm.nih.gov/pubmed/20955852

997 Yannakoulia M, Sitara M, Matalas AL. Reported eating behavior and attitudes improvement after a nutrition intervention program in a group of young female dancers. Int J Sport Nutr Exerc Metab. 2002. 12:24-32 https://www.ncbi.nlm.nih.gov/pubmed/11993620

$998 \quad$ Yildiran H, Mercanligil SM. Does increasing meal frequency improve weight loss and some biochemical parameters in overweight/obese females?. Nutr Hosp. 2019. 36:66-72 https://www.ncbi.nlm.nih.gov/pubmed/30836763

999 Yoncheva YN, Castellanos FX, Pizinger T, Kovtun K, St-Onge MP. Sleep and meal-time misalignment alters functional connectivity: a pilot resting-state study. Int J Obes (Lond). 2016. 40:1813-1816 https://www.ncbi.nlm.nih.gov/pubmed/27478925

Yoo KB, Suh HJ, Lee M, Kim JH, Kwon JA, Park EC. Breakfast eating patterns and the metabolic syndrome: the Korea National Health and Nutrition Examination Survey (KNHANES) 2007-2009. Asia Pac J Clin Nutr. 2014. 23:128-37 https://www.ncbi.nlm.nih.gov/pubmed/24561981

Study Design,

Intervention/Exposure

Daily Eating Occasions Not Reported, Eating Frequency Data Collection

Daily Eating Occasions Not Reported

Study Design

Intervention/Exposure, Daily Eating Occasions Not Reported

Eating Frequency Data Collection

Daily Eating Occasions Not Reported, Comparator, Size of Study Groups

Study Design 


\section{Citation}

1001

Yoshida J, Eguchi E, Nagaoka K, Ito T, Ogino K. Association of night eating habits with metabolic syndrome and its components: a longitudinal study. BMC Public Health. 2018. 18:1366

https://www.ncbi.nlm.nih.gov/pubmed/30537972

1002

Yoshimura E, Hatamoto Y, Yonekura S, Tanaka H. Skipping breakfast reduces energy intake and physical activity in healthy women who are habitual breakfast eaters: A randomized crossover trial. Physiol Behav. 2017. 174:89-94 https://www.ncbi.nlm.nih.gov/pubmed/28284879

1003 Yucel A, Degirmenci B, Acar M, Albayrak R, Haktanir A. The effect of fasting month of Ramadan on the abdominal fat distribution: assessment by computed tomography. Tohoku J Exp Med. 2004. 204:179-87 https://www.ncbi.nlm.nih.gov/pubmed/15502416

1004 Zakrzewski-Fruer JK, Plekhanova T, Mandila D, Lekatis Y, Tolfrey K. Effect of breakfast omission and consumption on energy intake and physical activity in adolescent girls: a randomised controlled trial. Br J Nutr. 2017. 118:392-400 https://www.ncbi.nlm.nih.gov/pubmed/28901889

1005 Zakrzewski-Fruer JK, Wells EK, Crawford NSG, Afeef SMO, Tolfrey K. Physical Activity Duration but Not Energy Expenditure Differs between Daily and Intermittent Breakfast Consumption in Adolescent Girls: A Randomized Crossover Trial. J Nutr. 2018. 148:236-244 https://www.ncbi.nlm.nih.gov/pubmed/29490105

1006 Zalewska M, Maciorkowska E. Dietary Habits and Physical Activity of 18-Year-Old Adolescents in Relation to Overweight and Obesity. Iran J Public Health. 2019. 48:864-872 https://www.ncbi.nlm.nih.gov/pubmed/31523643

1007 Zare A, Hajhashemi M, Hassan ZM, Zarrin S, Pourpak Z, Moin M, Salarilak S, Masudi S, Shahabi S. Effect of Ramadan fasting on serum heat shock protein 70 and serum lipid profile. Singapore Med J. 2011. 52:491-5 https://www.ncbi.nlm.nih.gov/pubmed/21808959

1008 Zarrouk N, Hammouda O, Latiri I, Adala H, Bouhlel E, Rebai H, Dogui M. Ramadan fasting does not adversely affect neuromuscular performances and reaction times in trained karate athletes. J Int Soc Sports Nutr. 2016. 13:18 https://www.ncbi.nlm.nih.gov/pubmed/27099568

1009 Zarrouk N, Hug F, Hammouda O, Rebai H, Tabka Z, Dogui M, Bouhlel E. Effect of Ramadan intermittent fasting on body composition and neuromuscular performance in young athletes: A pilot study. Biological Rhythm Research. 2013. 44:697-709

1010
Rationale

Daily Eating Occasions Not Reported

Daily Eating Occasions Not Reported, Outcome

Intervention/Exposure, Daily

Eating Occasions Not Reported

Daily Eating Occasions Not

Reported

Outcome

Daily Eating Occasions Not

Reported

Daily Eating Occasions Not Reported, Size of Study Groups

Daily Eating Occasions Not

Reported

Comparator, Size of Study Groups (almonds) on hunger, eating frequency, dietary intake and body weight. J Hum Nutr Diet. 2009. 22:461-8 https://www.ncbi.nlm.nih.gov/pubmed/19743983 
Ramadan fasting study. J Sports Sci. 2008. 26 Suppl 3:S3-6 https://www.ncbi.nlm.nih.gov/pubmed/19085447

1012 Zerva A, Nassis GP, Krekoukia M, Psarra G, Sidossis LS. Effect of eating frequency on body composition in 9-11year-old children. Int J Sports Med. 2007. 28:265-70 https://www.ncbi.nlm.nih.gov/pubmed/17024645

1013 Zhang L, Cordeiro LS, Liu J, Ma Y. The Association between Breakfast Skipping and Body Weight, Nutrient Intake, and Metabolic Measures among Participants with Metabolic Syndrome. Nutrients. 2017;9(4). https://www.ncbi.nlm.nih.gov/pubmed/28420112.

1014 Zhu Y, Hollis JH. Associations between eating frequency and energy intake, energy density, diet quality and body weight status in adults from the USA. Br J Nutr. 2016. 115:2138-44 https://www.ncbi.nlm.nih.gov/pubmed/27109636

Study Design

Ziaee V, Kihanidoost Z, Younesian M, Akhavirad M, Bateni F, Kazemianfar Z, Hantoushzadeh S. The effect of
Ramadan fasting on outcome of pregnancy. Iranian Journal of Pediatrics. 2010. 20:181-186

Ziaee V, Kihanidoost Z, Younesian M, Akhavirad M, Bateni F, Kazemianfar Z, Hantoushzadeh S. The effect of
Ramadan fasting on outcome of pregnancy. Iranian Journal of Pediatrics. 2010. 20:181-186 http://search.ebscohost.com/login. aspx?direct=true\&db=jlh\&AN=105055693\&site=ehost-live

1016 Ziaee V, Razaei M, Ahmadinejad Z, Shaikh H, Yousefi R, Yarmohammadi L, Bozorgi F, Behjati MJ. The changes of metabolic profile and weight during Ramadan fasting. Singapore Med J. 2006. 47:409-14 https://www.ncbi.nlm.nih.gov/pubmed/16645692

1017 Zimmerman AR, Ferriday D, Davies SR, Martin AA, Rogers PJ, Mason A, Brunstrom JM. "What time is my next meal?" delay-discounting individuals choose smaller portions under conditions of uncertainty. Appetite. 2017. 116:284-290 https://www.ncbi.nlm.nih.gov/pubmed/28501421

1018 Zimmerman AR, Johnson L, Brunstrom JM. Assessing "chaotic eating" using self-report and the UK Adult National Diet and Nutrition Survey: No association between BMI and variability in meal or snack timings. Physiol Behav. 2018. 192:64-71 https://www.ncbi.nlm.nih.gov/pubmed/29580954

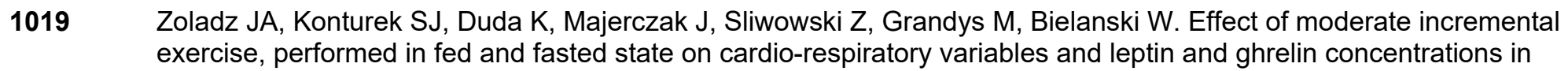
young healthy men. J Physiol Pharmacol. 2005. 56:63-85 https://www.ncbi.nlm.nih.gov/pubmed/15795476

1020 Zulet MA, Bondia-Pons I, Abete I, de la Iglesia R, Lopez-Legarrea P, Forga L, Navas-Carretero S, Martinez JA. The reduction of the metabolyc syndrome in Navarra-Spain (RESMENA-S) study: a multidisciplinary strategy based on chrononutrition and nutritional education, together with dietetic and psychological control. Nutr Hosp. 2011. 26:16-26 https://www.ncbi.nlm.nih.gov/pubmed/21519726

Daily Eating Occasions Not Reported

Eating Frequency Data Collection

Daily Eating Occasions Not Reported

Intervention/Exposure, Daily Eating Occasions Not Reported

Intervention/Exposure, Daily Eating Occasions Not Reported

Study Design

Intervention/Exposure

Study Design 
